\title{
Spectrum and Energy Levels of Neutral Thulium
}

\author{
J. Sugar and W. F. Meggers* \\ Institute for Basic Standards, National Bureau of Standards, Washington, D.C. 20234 \\ and \\ P. Camus
}

Laboratoire Aimé Cotton, Centre National de la Recherche Scientifique, 91 -Orsay, France

(July 11, 1972)

\begin{abstract}
A new set of measurements of the spectrum of neutral thulium covering the range of $2513 \AA$ to $11750 \AA$ is given. The light source utilized for the observations was a microwave-excited thuliumiodide lamp. Second spectrum lines were recognized by comparing the output of this lamp with that of a 6A sliding spark. The results of Zeeman effect studies of these lines are included. Analysis of these new dat a led to the discovery of 348 new energy levels and provided revised values for the 142 already published. Measured Landé $g$ factors are given for most of the levels.
\end{abstract}

Key words: Energy levels; spectrum; thulium; wavelengths.

\section{Introduction}

The fundamental energy interval in neutral thulium of $8771 \mathrm{~cm}^{-1}$ was discovered by Meggers in 1942 [1].1 He identified it as the ${ }^{2} \mathrm{~F}$ interval of the $4 f^{13} 6 s^{2}$ normal electronic configuration. Sixteen pairs of lines were found with this wave number difference, giving as many upper levels. The line list was obtained by comparing spectra of $\mathrm{Tm}, \mathrm{Yb}$, and $\mathrm{Lu}$ oxides, each containing the other two as considerable impurities. Although the availability of the pure metal by the middle 1950's allowed spectroscopic studies to be made much more easily and completely, it was not until 1963 that the first new results were obtained $[2,3]$ by Blaise and co-workers at Laboratoire Aimé Cotton. They studied the $h f s$ of 70 strong lines utilizing a cooled hollow cathode light source and a Fabry-Perot spectrometer. Their investigation confirmed the 16 levels of even parity found by Meggers, assigned $J$ values to them, and extended the analysis to include ten high-lying levels of odd parity (including the discovery of the $4 f^{13} 6 s 7 s$ configuration), as well as 12 new even levels.

At about this time the work of making a completely new description of the spectra of thulium was initiated at the National Bureau of Standards in Washington, D.C. The program was to make as complete as practical a set of wavelengths of the first and second spec-

\footnotetext{
* Deceased.

${ }^{1}$ Figures in brackets indicate the literature references at the end of this paper.
}

tra in the range of $2000 \AA$ to $12000 \AA$. This was to be done with the microwave-excited metal-halide lamp and the low current sliding spark, the distinction between first and second spectra being provided by their relative enhancement in these two light sources. The work, which occupied a span of three years, resulted in the measurement of a set of 4378 lines of Tm I and 12842 lines of Tm II with an accuracy of about $0.03 \mathrm{~cm}^{-1}$. In addition, there appeared in the spark 848 lines of higher ionization which were identified as such by comparison with the spectrum of a higher current spark. Of these, 454 have been classified in a level scheme of doubly ionized thulium [4].

Two new sets of observations acquired by Laboratoire Aimé Cotton led to the next breakthrough in the analysis of Tm I: spectrograms of the Zeeman effect taken at the Argonne National Laboratory and a line list extending from $0.9 \mu \mathrm{m}$ to $2.4 \mu \mathrm{m}$ obtained from observations made with the S.I.S.A.M. spectrometer $[5]$. These data enabled the assignment of $J$ values and $g$ values to nearly every known level and led to the discovery of 42 new even levels and 62 new odd levels [5-7].

The information on Tm I and Tm II was now sufficient to permit all the low-lying configurations to be interpreted through parametric calculations of the energy levels, eigenvectors, and $g$ values. These calculations were carried out by Camus [8] for the mixed configurations $4 f^{12} 5 d 6 s^{2}$ and $4 f^{13} 6 s 6 p$ of even parity, and for $4 f^{12} 6 s^{2} 6 p$ and $4 f^{13} 5 d 6 s$ of odd parity [9]. The 
simple configurations $4 f^{13} 6 s 7 s$ and $4 f^{13} 6 s 8 s$ had already been interpreted and utilized in a determination of the ionization energy in reference [7].

With the completion of the measurements of Tm I and Tm II at the National Bureau of Standards, a collaboration was established with Laboratoire Aimé Cotton to pool resources and to search for the higher-lying energy levels of Tm. Part of the fruits of this labor are presented here. The new results in Tm I consist mainly of the discovery and interpretation of the levels belonging to the $4 f^{13} 5 d 6 p, 4 f^{12} 5 d 6 s 6 p$, $4 f^{13} 6 s 6 d$, and $4 f^{13} 6 p^{2}$ configurations as well as of additional levels belonging to the known configurations mentioned above. The known even levels were augmented by 138 additions, and the odd levels by 210 with the position of all the energy levels based on the new wavelength measurements.

We present here all the previously published energy levels of Tm I as well as those new ones found with the NBS line list and the Zeeman data, their measured $g$ values, and their designations obtained from the parametric calculations [23]. The line list of Tm I measured at NBS is given in its entirety, with classifications and Zeeman data given where known. The results of the calculations of the newly identified configurations will be given in a later publication.

\section{Observations and Measurements}

The microwave-excited metal-halide lamp was used as the light source for the first spectrum of thulium, both for the wavelength measurements at the National Bureau of Standards and for the Zeeman effect measurements at the Argonne National Laboratories. A description of the method of preparation and the desirable features of these lamps has been given in detail $[10,11]$. Because this light source produces a considerable excitation of singly ionized thulium, a clear distinction between the spectra of the neutral and singly ionized species must be made. This was accomplished unambiguously for practically all lines by comparing the lamp spectrum with that obtained by means of a low-current, low-voltage sliding spark. The versatility of this spark (described in detail in ref. [12]) in separating various ionic spectra by variations of the peak discharge current was previously demonstrated in studies of the third spectra of praseodymium [12] and cerium [13]. For the Pr III work it was operated at $50 \mathrm{~A}$ and $1000 \mathrm{~A}$ to produce relatively enhanced third or fourth spectrum. The lines of Pr II were distinguished by comparison with an arc spectrum of Pr. In the cerium work the spark was found to operate well at $6 \mathrm{~A}$ peak current and to produce a second spectrum which was comparable to the arc spectrum in relative enhancement but much sharper. A line width of $\sim 0.1 \mathrm{~cm}^{-1}$ is obtained with the sliding spark at 6A peak current. For our analysis the spectrum obtained in this operating condition was compared with that of the metal-halide lamp to pick out second spectrum lines and to provide the line list of Tm II.

A variety of spectrographs was used to obtain the wavelength coverage for our observations. From 2000 to $2600 \AA$ a Wadsworth mounting of a $6.4 \mathrm{~m}$ grating with 1200 grooves $/ \mathrm{mm}$ was used in the third order giving a plate factor of $0.6 \AA / \mathrm{mm}$ and in second order from 2600 to $4400 \AA$. The data from 4400 to $6850 \AA$ was obtained with an Eagle mounting of a 10.7 m grating having 600 grooves $/ \mathrm{mm}$ utilized in the second order. Averaged in with the data from the Eagle spectrograph are some measurements in first order of the 6.4 m Wadsworth. Two more Wadsworth mountings were used to carry the observations to $12000 \AA$ : one containing a $6.4 \mathrm{~m}$ grating with 600 grooves $/ \mathrm{mm}$ and blazed at $9500 \AA$, and the second with a grating of the same radius of curvature but with 300 grooves $/ \mathrm{mm}$ and a blaze at $12000 \AA$. This same spectral range is now covered at NBS by two Eagle mountings, one in vacuum and one in air, with 10.7 m gratings.

The observations of the Zeeman effect were made using the Paschen-Runge mounting of a $9.15 \mathrm{~m}$ grating at the Argonne National Laboratory and using a magnetic field of $\sim 24000$ Oe. The construction and operation of this excellent facility is described in two papers [14, 15] by Tomkins and Fred, who generously provided the thulium exposures for us.

Maximum exposure times for the Tm lamp varied from $1 \mathrm{~h}$ in the ultraviolet below $3000 \AA$ to $10 \mathrm{~min}$ in the visible and to $3 \mathrm{~h}$ in the extreme photographic infrared. Shorter exposures were, of course, used to obtain the stronger lines with good images. Exposure times for the $6 \mathrm{~A}$ spark followed a similar pattern with maxima of 3 to $5 \mathrm{~h}$ in the extreme wavelength regions to $5 \mathrm{~min}$ in the visible. A spark repetition rate of about $20 / \mathrm{s}$ was used with a rotating external spark gap placed in the series discharge circuit. The $50 \mathrm{~A}$ spark operating at one-third of the exposure time of the $6 \mathrm{~A}$ spark produced weaker second spectrum and stronger third or higher spectra, thus giving the desired separation.

Calibration standards in the region of 2000 to $3000 \AA$ were obtained from a copper hollow-cathode discharge containing a few grams of germanium supplemented by an iron-iodide lamp which provided $\mathrm{Fe} \mathrm{I}$ and $\mathrm{Fe}$ II in this region. The copper standard wavelengths used were the interferometric measurements of Reader, Meissner, and Andrew [16]; the germanium measurements in this region are those of Van Veld and Meissner [17]. Since these lines are fairly sparsely distributed in the region of 2000 to $2600 \AA$, the calibration was augmented by Fe II lines measured by Meggers and Humphreys [18]. No shift was detectable among these spectra. From 2600 to $3300 \AA$ interferometrically measured lines of $\mathrm{Fe} I$ were used [19]. The longer wavelength regions were well covered in first or second order by the thorium measurements of Meggers and Stanley [20] which were available prior to publication.

A pulsed high-current arc in $\mathrm{He}$ at $\mathrm{l}$ atm was used as a source to aid in the recognition of resonance and other low-excitation lines of Tm I and Tm II [21]. It produced self-reversal of 120 first spectrum lines and 126 of the second spectrum. The self-reversals are two types: one for which there appears only a gap in the continuum with little trace of a line left (designated 
" $a$ " in the line list under "intensity"), and a second type for which most of the wings of the lines still remain (designated " $r$ "). Eleven such lines remain unclassified. The classified ones arise mainly from the ground ${ }^{2} \mathrm{~F}$ term, but some have the low level as high as $19466 \mathrm{~cm}^{-1}$. All such low levels for these lines were found to be metastable because of their $J$ values.

Each spectral line included in the list has been measured on at least two separate plates, and on as many as ten for some of the stronger lines. We found that 95 percent of the classified lines fit the energy level scheme to within $\pm 0.05 \mathrm{~cm}^{-1}$. A measure of the wave number precision was made by finding the rms deviation in repeating wave number differences determining the low-lying level intervals. For the 27 pairs determining the lowest interval of 8771.243 $\mathrm{cm}^{-1}$, the rms deviation is $0.02 \mathrm{~cm}^{-1}$. Intervals between the level pairs of even parity $13119.610-15587.811$ $\mathrm{cm}^{-1}$ and $15587.811-17454.818 \mathrm{~cm}^{-1}$ having 59 and 51 line pairs, respectively, are determined with $\mathrm{rms}$ deviations of $0.03 \mathrm{~cm}^{-1}$ each. Therefore, the $\mathrm{rms}$ error in the individual wave number measurements is estimated to be $0.03 / 1.4=0.02 \mathrm{~cm}^{-1}$.

A total of 2734 lines are classified, and, of these, 47 are classified twice. These are mostly weak lines with no observed Zeeman effect. The complete line list is given in the appendix which includes the measured wavelengths, visually estimated relative intensities from 1 to 50,000 , vacuum wave numbers, classifications of the lines, and measured Zeeman effect. The intensity numbers are sometimes followed by letters indicating the following characteristics of the lines:

$$
\begin{aligned}
h & =\text { hazy } \\
d & =\text { a double line } \\
w & =\text { wide } \\
r & =\text { reversed in pulsed arc } \\
a & =\text { nearly completely absorbed in pulsed arc. }
\end{aligned}
$$

The classifications contain the values of the energy levels responsible for the transition, their $J$ values given as subscripts, and a superscript degree symbol to indicate odd parity. The column headed "Zeeman Effect" generally contains the three following pieces of information:

1. The type of Zeeman pattern observed which is indicated by the symbols:

$\Delta J=0$ where the $p$-components are shaded toward the center,

SI where the $n$-components are shaded toward the center,

$S O$ where the $n$-components are shaded away from the center,

SOO where $n$-components are shaded away from center and are overlapping,

$T$ where only a triplet was observed.

2. Half the interval between the two corresponding strong $n$-components in Lorentz units, denoted by " $f$ ".

3. Half the interval between the two outer $p$-com- ponents in Lorentz units, denoted by "Je" where " $e$ " is the interval between components $(e=\Delta g)$.

When the pattern is completely resolved. the two derived $g$ factors are given in the columns " $f$ " and "Je" with star $\left(^{*}\right)$ in the "Je" column to indicate such cases. The limit of resolution for " $e$ " was 0.05 , and the uncertainty in the $g$ factors is estimated to be \pm 0.01 .

\section{Energy Levels}

Neutral thulium is one of the simpler rare earths at low energy because of its nearly closed $4 f$ shell. The ground configuration, $4 f^{13} 6 s^{2}$, consists of two levels with a separation of $8771 \mathrm{~cm}^{-1}$. Above $13119 \mathrm{~cm}^{-1}$ the level structure begins to exhibit a complex character resulting from excitation of an electron from the $4 f$ into the $5 d$ shell, and at somewhat higher energy, the excitation of a $6 s$ electron into the $6 p$ shell. The resulting overlapping configurations of even parity, $4 f^{125} d 6 s^{2}$ and $4 f^{13} 6 s 6 p$, are heavily mixed and form a dense complex of levels. There is a corresponding low energy complex of levels of odd parity beginning at $20406 \mathrm{~cm}^{-1}$ formed by one-electron excitations out of the ground configuration. They arise from the configurations $4 f^{13} 5 d 6 s$ and $4 f^{12} 6 s^{2} 6 p$. Because these two low-lying complex groups of levels of opposite parity can combine by one-electron transitions, they produce a strong spectrum in the infrared.

A diagram showing the start of each of the known configurations is given in figure 1. Transitions in the infrared are indicated by heavy lines while those in the visible and shorter wavelength regions are indicated by the fine lines. The arrows show the order of discovery of the various configurations as the analysis progressed. Strong configuration interaction in the two low groups accounts for the observation of "forbidden" transitions $4 f^{12} 6 s^{2} 6 p-4 f^{13} 6 s 6 p$ and $4 f^{12} 5 d 6 s^{2}-$ $4 f^{13} 5 d 6 s$.

The next stage of complexity begins at the threshold for 2-electron excitations out of the ground configuration. The observed odd configuration of this type, $4 f^{12} 5 d 6 s 6 p$, accounts for the bulk of the known levels of this parity. Levels of this configuration begin at $29308 \mathrm{~cm}^{-1}$ and extend far beyond the ionization energy. Within their bounds were found four much simpler configurations: $4 f^{13} 6 s 7 s, 4 f^{13} 6 s 8 s, 4 f^{13} 6 s 6 d$, and $4 f^{13} 6 p^{2}$.

No even configuration of comparable complexity has been found, but Brewer [22] predicted the presence of $4 f^{12} 5 d^{2} 6 s$ at about $27600 \mathrm{~cm}^{-1}$. In this parity there are many known high-lying levels without interpretation. It is likely that they belong to this configuration, but they cannot be understood as such until the low structure of this configuration is discovered. The limitation here is that $4 f^{12} 5 d^{2} 6 s$ lies so close to $4 f^{12} 5 d 6 s 6 p$ that the strong transitions are in the far infrared. The simpler higher-lying even configuration $4 f^{13} 5 d 6 p$ has been identified at $38318 \mathrm{~cm}^{-1}$.

Many higher-lying energy levels of even parity based on single lines seen in photoabsorption have been 


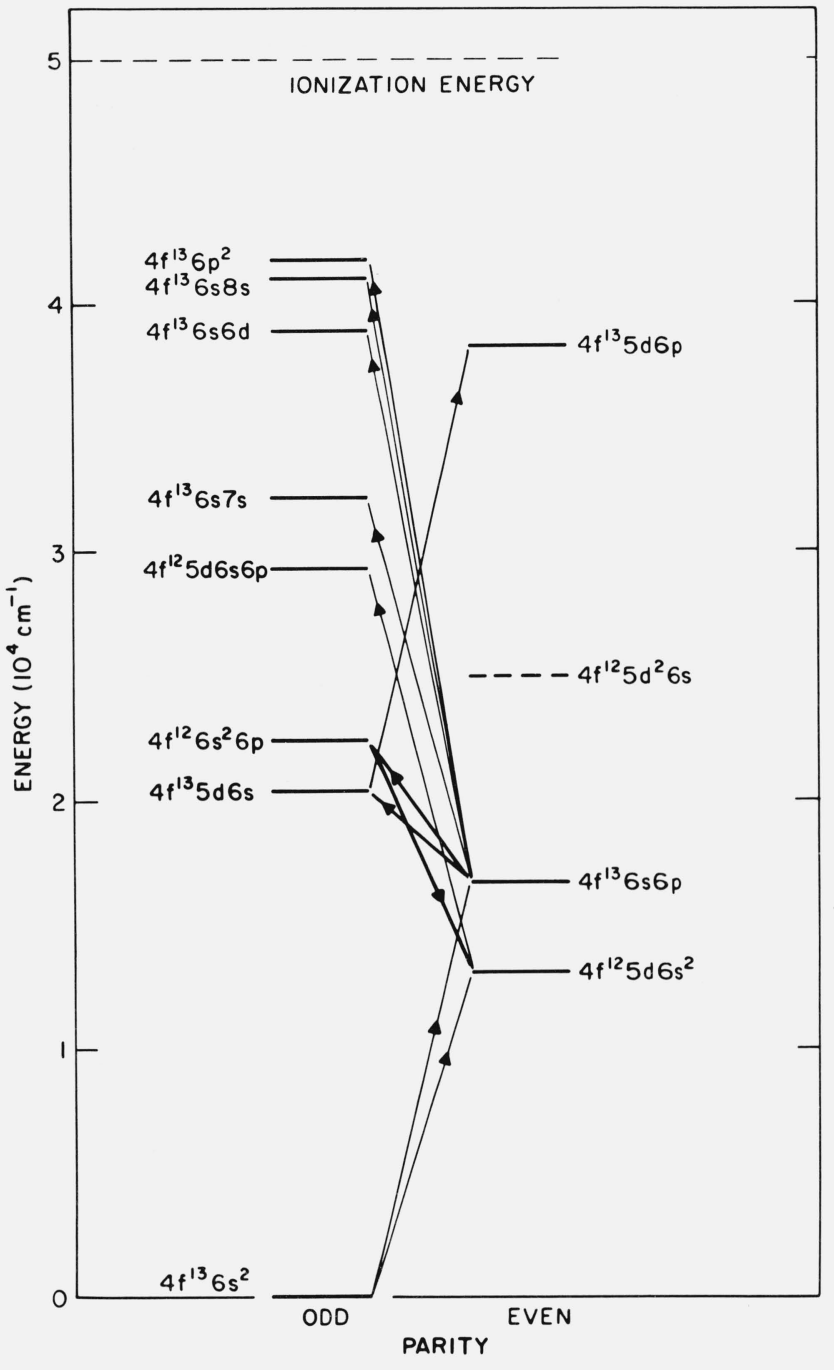

FIGURE 1. Relative positions of known configurations of neutral thulium, with each configuration represented by lowest level.

Dashed line gives predicted position for $4 f^{12} 5 d^{2} 6 s$. Arrows show the order of discovery of the configurations. Heavy arrows indicate where infrared lines were used.

reported by Camus [23]. He was able to follow the $4 f^{13} 6 s n p$ series from $n=17$ to $n=48$. Many of the levels reported here are supported by the absorption data, but we include no levels which are not in evidence in emission.

Table I contains all levels of Tm I of even parity which are deduced from the present line list. Nearly all of those below $35000 \mathrm{~cm}^{-1}$ have been reported before. Their source is indicated by a number in the last column denoting the reference appearing in section 5. The levels of the three configurations identified are grouped into $J_{1} J_{2}$ coupling terms. For $4 f^{12} 5 d 6 s^{2}$ and $4 f^{13} 6 s 6 p$ the designations are taken from reference [8]. The designations of $4 f^{13} 5 d 6 p$ are from calculations which we will soon publish.

Table 2 contains all the known levels of odd parity. The configurations $4 f^{12} 6 s^{2} 6 p, 4 f^{12} 5 d 6 s 6 p, 4 f^{13} 6 s 7 s$, $4 f^{13} 6 s 8 s$, and $4 f^{13} 6 p^{2}$, are designated in $J_{1} J_{2}$ coupling as indicated by the parametric treatment $[9,23]$. The coupling scheme applied to the configurations $4 f^{13} 5 d 6 s$ and $4 f^{13} 6 s 6 d$ is $J_{1} L_{2}$ coupling. The $J_{1}$ vector refers to the $4 f^{13}$ angular momentum, and $L_{2}$ denotes the orbital angular momentum of the $d s$ system. The resultant angular momentum $\mathrm{K}$ is enclosed in a square bracket with a superscript on the left of the bracket denoting the spin of the $d s$ system. Those few odd levels which have not been assigned to a configuration appear in numerical order in the total list. Many levels of $4 f^{12} 5 d 6 s 6 p$ have only a configuration assignment because of technical limitations in the theoretical treatment of this extremely large configuration. These limitations will be discussed in a later paper on the theoretical treatment of the high configurations, including the $4 f^{13} 6 s 6 d$ and $4 f^{13} 6 p^{2}$ and $4 f^{13} 5 d 6 p$. Designations for levels of the configurations $4 f^{12} 6 s^{2} 6 p$ and $4 f^{135} d 6 s$ are taken from references [5] and [9]. The sources for levels previously published are denoted as in table 1 .

The level values for both parities were adjusted by least squares fitting to the measured lines with a computer code written by Radziemski, Fisher, Steinhaus, and Goldman for this purpose [24]. For levels given to three decimal places the standard deviation varies from 0.004 to $0.010 \mathrm{~cm}^{-1}$. For those given to two it varies from 0.010 to $0.02 \mathrm{~cm}^{-1}$. The $\mathrm{rms}$ deviation of the measured lines from the spectrum calculated with the adjusted level values is $0.02 \mathrm{~cm}^{-1}$. 
TABLE 1. Energy levels of neutral thulium, even parity, in units of $\mathrm{cm}^{-1}$. Ref. is given for levels previously published

\begin{tabular}{|c|c|c|c|c|c|c|c|c|c|c|c|}
\hline Configuration & $\begin{array}{l}\text { Desig- } \\
\text { nation }\end{array}$ & $J$ & Energy & $g$-Factor & Ref. & Configuration & $\begin{array}{l}\text { Desig- } \\
\text { nation }\end{array}$ & $J$ & Energy & $g$-Factor & Ref. \\
\hline \multirow[t]{4}{*}{$4 f^{12}\left({ }^{3} \mathrm{H}_{6}\right) 5 d_{3 / 2} 6 s^{2}$} & $\left(6,1^{1 / 2}\right)$ & $4^{1 / 2}$ & 13119.610 & 1.305 & 6 & \multirow{5}{*}{$4 f^{13}\left({ }^{2} \mathrm{~F}_{5 / 2}\right) 6 s 6 p\left({ }^{3} \mathrm{P}_{2}\right)$} & \multirow[t]{5}{*}{$\left(2^{1 / 2}, 2\right)$} & $0^{1 / 2}$ & 27314.56 & -0.015 & 6 \\
\hline & & $7^{1 / 2}$ & 15271.002 & 1.08 & 5 & & & $4^{1 / 2}$ & 28024.01 & 1.15 & 6 \\
\hline & & $5^{1 / 2}$ & 15587.811 & 1.255 & 7 & & & $1^{1 / 2}$ & 28143.67 & 0.66 & 6 \\
\hline & & $6^{1 / 2}$ & 17454.818 & 1.15 & 7 & & & $2^{1 / 2}$ & 28448.585 & 1.000 & 6 \\
\hline \multirow{6}{*}{$4 f^{12}\left({ }^{3} \mathrm{H}_{6}\right) 5 d_{5 / 2} 6 s^{2}$} & $\left(6,2^{1 / 2}\right)$ & $8^{1 / 2}$ & 16456.913 & 1.175 & 5 & & & $3^{1 / 2}$ & 28555.799 & 1.157 & 6 \\
\hline & & $3^{1 / 2}$ & 16957.006 & 1.169 & 2 & \multirow[t]{3}{*}{$4 f^{2}\left({ }^{3} \mathrm{H}_{4}\right) 5 d_{3 / 2} 6 s^{2}$} & \multirow[t]{3}{*}{$\left(4,1^{1 / 2}\right)$} & $2^{1 / 2}$ & 28051.37 & 0.96 & 1 \\
\hline & & $7^{1 / 2}$ & 18693.074 & 1.18 & 5 & & & $4^{1 / 2}$ & 29316.690 & 0.978 & 6 \\
\hline & & $4^{1 / 2}$ & 18837.385 & 1.13 & 2 & & & $3^{1 / 2}$ & 31510.24 & 1.11 & 1 \\
\hline & & $5^{1 / 2}$ & 18853.823 & 1.15 & 6 & \multirow{4}{*}{$4 f^{12}\left({ }^{3} \mathrm{~F}_{3}\right) 5 d_{3 / 2} 6 s^{2}$} & \multirow[t]{4}{*}{$\left(3,1^{1 / 2}\right)$} & $3^{1 / 2}$ & 29260.59 & 0.928 & 6 \\
\hline & & $6^{1 / 2}$ & 19466.663 & 1.15 & 7 & & & $2^{1 / 2}$ & 31431.88 & 1.07 & 1 \\
\hline $4 f^{13}\left({ }^{2} \mathrm{~F}_{\tau_{2 / 2}}\right) 6 s 6 p\left({ }^{3} \mathrm{P}_{0}\right)$ & $\left(3^{1 / 2}, 0\right)$ & $3^{1 / 2}$ & 16742.237 & 1.325 & 2 & & & $4^{1 / 2}$ & 31440.54 & 1.13 & 7 \\
\hline \multirow{3}{*}{$4 f^{13}\left({ }^{2} \mathrm{~F}_{7 / 2}\right) 6 s 6 p\left({ }^{3} \mathrm{P}_{1}\right)$} & $\left(3^{1 / 2}, 1\right)$ & $3^{1 / 2}$ & 17343.374 & 1.022 & 2 & & & $1^{1 / 2}$ & 31520.98 & 0.79 & 6 \\
\hline & & $4^{1 / 2}$ & 17613.659 & 1.188 & 2 & \multirow{3}{*}{$4 f^{12}\left({ }^{3} \mathrm{~F}_{2}\right) 5 d_{3 / 2} 6 s^{2}$} & \multirow[t]{3}{*}{$\left(2,1^{1 / 2}\right)$} & $2^{1 / 2}$ & 30082.18 & 0.81 & 6 \\
\hline & & $2^{1 / 2}$ & 17752.634 & 1.186 & 2 & & & $1^{1 / 2}$ & 30585.65 & 1.085 & 7 \\
\hline \multirow{5}{*}{$4 f^{13}\left({ }^{2} \mathrm{~F}_{\tau / 2}\right) 6 s 6 p\left({ }^{3} \mathrm{P}_{2}\right)$} & $\left(3^{1 / 2}, 2\right)$ & $5^{1 / 2}$ & 18990.406 & 1.215 & 6 & & & $3^{1 / 2}$ & 32446.26 & 0.89 & 1 \\
\hline & & $1^{1 / 2}$ & 19132.245 & 0.88 & 6 & \multirow{4}{*}{$4 f^{12}\left({ }^{3} \mathrm{H}_{4}\right) 5 d_{5 / 2} 6 s^{2}$} & \multirow[t]{5}{*}{$\left(4,2^{1 / 2}\right)$} & $3^{1 / 2}$ & 30124.02 & 0.955 & 6 \\
\hline & & $2^{1 / 2}$ & 19548.834 & 0.983 & 2 & & & $6^{1 / 2}$ & 30125.61 & & \\
\hline & & $4^{1 / 2}$ & 19748.543 & 1.29 & 6 & & & $2^{1 / 2}$ & 30302.42 & 0.91 & 1 \\
\hline & & $31 / 2$ & 19753.830 & 1.18 & 2 & & & $4^{1} / 2$ & 30915.02 & 0.99 & 6 \\
\hline \multirow{4}{*}{$4 f^{12}\left({ }^{3} \mathrm{~F}_{4}\right) 5 d_{3 / 2} 6 s^{2}$} & $\left(4,1^{1 / 2}\right)$ & $3^{1 / 2}$ & 21120.836 & 1.175 & 2 & \multirow{4}{*}{$4 f^{12}\left({ }^{3} \mathrm{~F}_{3}\right) 5 d_{5 / 2} 6 s^{2}$} & & $5^{1 / 2}$ & 30972.46 & & \\
\hline & & $2^{1 / 2}$ & 21161.401 & 1.14 & 1 & & \multirow[t]{4}{*}{$\left(3,2^{1 / 2}\right)$} & $2^{1 / 2}$ & 32174.49 & 1.16 & 1 \\
\hline & & $4^{1 / 2}$ & 21737.685 & 1.13 & 2 & & & $4^{1 / 2}$ & 32407.78 & & \\
\hline & & $5^{1 / 2}$ & 21997.473 & 1.06 & 5 & & & $31 / 2$ & 32811.02 & 1.04 & 1 \\
\hline \multirow[t]{4}{*}{$4 f^{12}\left({ }^{3} \mathrm{H}_{5}\right) 5 d_{3 / 2} 6 s^{2}$} & $\left(5,1^{1 / 2}\right)$ & $3^{1 / 2}$ & 22791.176 & 1.14 & 1 & \multirow{4}{*}{$4 f^{12}\left({ }^{3} \mathrm{~F}_{2}\right) 5 d_{5 / 2} 6 s^{2}$} & & $1^{1 / 2}$ & 32928.76 & 0.935 & 6 \\
\hline & & $6^{1 / 2}$ & 23374.681 & 0.98 & & & \multirow[t]{3}{*}{$\left(2,2^{1 / 2}\right)$} & $1^{1 / 2}$ & 33489.36 & 1.468 & 6 \\
\hline & & $4^{1 / 2}$ & 24348.692 & 1.12 & 2 & & & $3^{1 / 2}$ & 33623.78 & 0.92 & 6 \\
\hline & & $5^{1 / 2}$ & 25488.545 & & & & & $2^{1 / 2}$ & 35026.22 & 1.022 & 6 \\
\hline $4 f^{12}\left({ }^{3} \mathrm{~F}_{4}\right) 5 d_{5 / 2} 6 s^{2}$ & $\left(4,2^{1 / 2}\right)$ & $2^{1 / 2}$ & 22929.717 & 1.152 & 1 & $4 f^{13}\left({ }^{2} \mathrm{~F}_{5 / 2}\right) 6 s 6 p\left({ }^{1} \mathrm{P}_{1}\right)$ & $\left(2^{1 / 2}, 1\right)$ & $2^{1 / 2}$ & 34085.20 & 0.95 & 1 \\
\hline & & $5^{1 / 2}$ & 23309.979 & 1.11 & 5 & & & $3^{1 / 2}$ & 34297.17 & 0.915 & 1 \\
\hline & & $4^{1 / 2}$ & 23781.698 & 1.14 & 2 & & & $1^{1 / 2}$ & 35089.67 & 0.85 & 6 \\
\hline & & $3^{1 / 2}$ & 23873.207 & 1.135 & 2 & & & $2^{1 / 2}$ & 35261.762 & 1.346 & \\
\hline & & $1^{1 / 2}$ & 23882.41 & & & & & $2^{1 / 2}$ & 37064.18 & 1.42 & \\
\hline & & $6^{1 / 2}$ & 24137.196 & 1.128 & 5 & & & $1^{1 / 2}$ & 37138.57 & 1.56 & \\
\hline $4 f^{13}\left({ }^{2} \mathrm{~F}_{7 / 2}\right) 6 s 6 p\left({ }^{1} \mathrm{P}_{1}\right)$ & $\left(3^{1 / 2}, 1\right)$ & $2^{1 / 2}$ & 24418.018 & 1.06 & 2 & & & $4^{1 / 2}$ & 37576.866 & 1.19 & \\
\hline & & $3^{1 / 2}$ & 26701.325 & 1.076 & 3 & & & $\begin{array}{l}41 / 2 \\
21 / 0\end{array}$ & 37711.074 & 1.14 & \\
\hline & & $4^{1 / 2}$ & 26889.125 & 1.11 & 3 & & & $\begin{array}{l}2^{1 / 2} \\
4^{1 / 2}\end{array}$ & $\begin{array}{l}37724.84 \\
37946.39\end{array}$ & & \\
\hline $4 f^{12}\left({ }^{3} \mathrm{H}_{5}\right) 5 d_{5 / 2} 6 s^{2}$ & $\left(5,2^{1} / 2\right)$ & $7^{1 / 2}$ & 25130.453 & 1.10 & & $4 f^{12}\left({ }^{1} \mathrm{G}_{4}\right) 5 d_{5 / 2} 6 s^{2}$ & $\left(4,2^{1 / 2}\right)$ & $2^{1 / 2}$ & 38014.37 & 1.11 & \\
\hline & & $3^{1 / 2}$ & 25717.197 & 1.02 & 1 & & & $3^{1 / 2}$ & 38342.57 & 1.108 & \\
\hline & & $2^{1 / 2}$ & 25745.117 & 1.09 & 6 & & & $1^{1 / 2}$ & 39514.74 & 0.80 & \\
\hline & & $4^{1 / 2}$ & 26646.214 & 0.961 & 3 & & & $4^{1 / 2}$ & 39768.79 & & \\
\hline & & $\begin{array}{l}6^{1 / 2} \\
5^{1 / 2}\end{array}$ & $\begin{array}{l}27377.13 \\
27491.31\end{array}$ & 1.12 & & $4 f^{12}\left({ }^{1} \mathrm{G}_{4}\right) 5 d_{3 / 2} 6 s^{2}$ & $\left(4,1^{1 / 2}\right)$ & $4^{1 / 2}$ & 38120.71 & & \\
\hline & & & 26491.31 & & & & & $3^{1 / 2}$ & 39560.41 & 0.895 & 7 \\
\hline $4 f^{13}\left({ }^{2} \mathrm{~F}_{5 / 2}\right) 6 s 6 p\left({ }^{3} \mathrm{P}_{0}\right)$ & $\left(2^{1 / 2}, 0\right)$ & $2^{1 / 2}$ & 25656.019 & 0.724 & 1 & & & $2^{1 / 2}$ & 40787.24 & 0.91 & 6 \\
\hline $4 f^{13}\left({ }^{2} \mathrm{~F}_{5 / 2}\right) 6 s 6 p\left({ }^{3} \mathrm{P}_{1}\right)$ & $\left(2^{1 / 2}, 1\right)$ & $2^{1 / 2}$ & 26126.907 & 1.10 & 1 & & & $3^{1 / 2}$ & 38123.02 & 1.14 & \\
\hline & & $3^{1 / 2}$ & 26439.491 & 1.000 & 1 & & & $2^{1 / 2}$ & 38128.37 & 1.05 & \\
\hline & & $1^{1 / 2}$ & 26488.70 & 0.85 & 6 & & & $1^{1 / 2}$ & 38237.89 & 1.04 & \\
\hline
\end{tabular}


TABLE 1. Energy levels of neutral thulium, even parity, in units of $\mathrm{cm}^{-1}$. Ref. is given for levels previously published-Continued

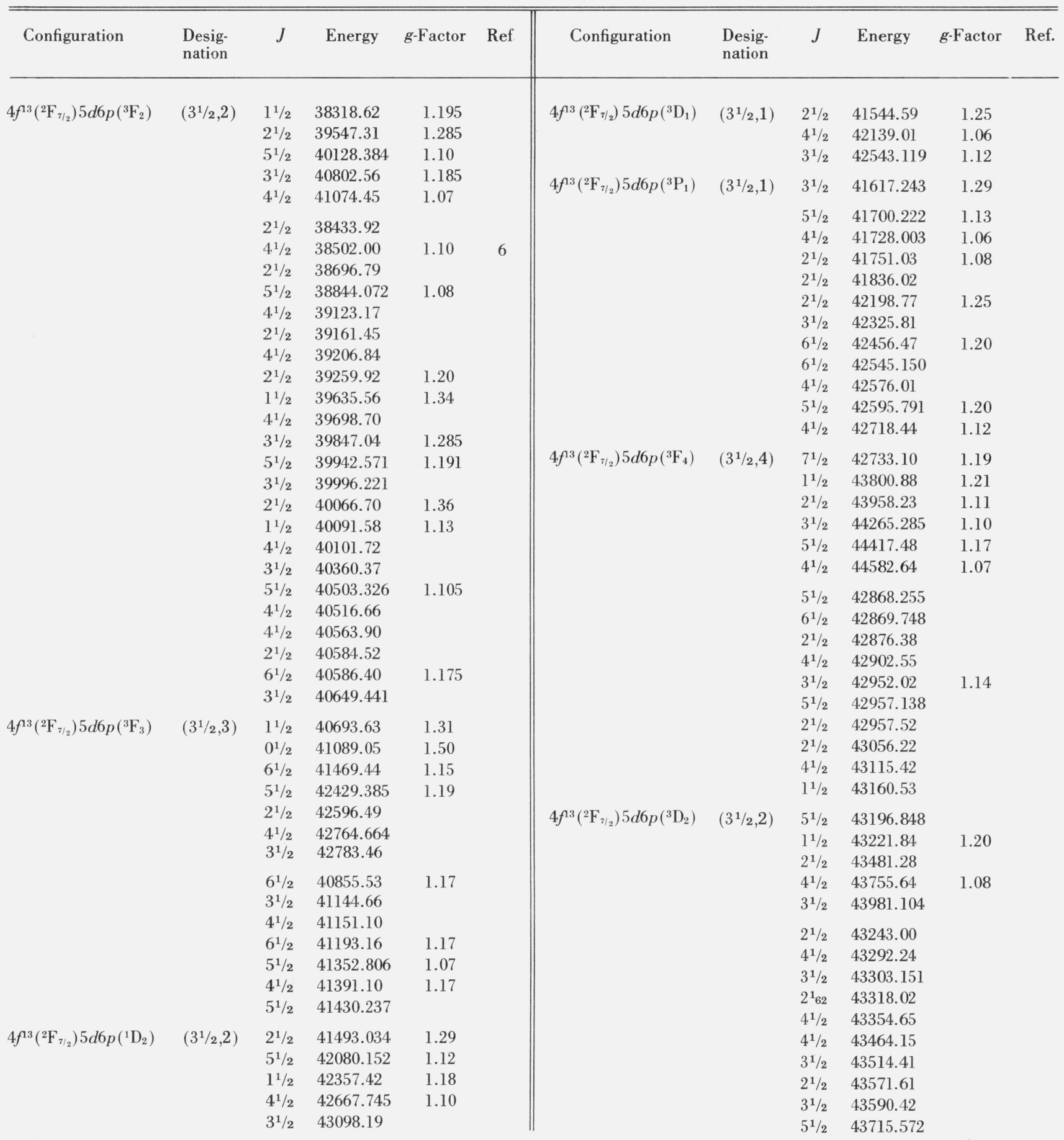


TABLE 1. Energy levels of neutral thulium, even parity, in units of $\mathrm{cm}^{-1}$. Ref. is given for levels previously published-Continued

\begin{tabular}{|c|c|c|c|c|c|c|c|c|c|c|}
\hline Configuration & $\begin{array}{l}\text { Desig- } \\
\text { nation }\end{array}$ & $J$ & Energy & $g$-Factor Ref. & Configuration & $\begin{array}{l}\text { Desig- } \\
\text { nation }\end{array}$ & $J$ & Energy & $g$-Factor & Ref. \\
\hline $4 f^{13}\left({ }^{2} \mathrm{~F}_{7 / 2}\right) 5 d 6 p\left({ }^{3} \mathrm{D}_{3}\right)$ & $\left(3^{1 / 2}, 3\right)$ & $\begin{array}{l}5^{1 / 2} \\
6^{1 / 2} \\
4^{1 / 2} \\
2^{1 / 2} \\
1^{1 / 2} \\
3^{1 / 2} \\
2^{1 / 2} \\
3^{1 / 2} \\
4^{1 / 2} \\
2^{1 / 2} \\
5^{1 / 2} \\
3^{1 / 2} \\
6^{1 / 2} \\
3^{1 / 2} \\
4^{1 / 2} \\
3^{1 / 2} \\
4^{1 / 2} \\
3^{1 / 2} \\
4^{1 / 2}\end{array}$ & $\begin{array}{l}43788.44 \\
43836.666 \\
44380.90 \\
44518.91 \\
44545.90 \\
44750.078 \\
43859.06 \\
44206.39 \\
44238.85 \\
44243.40 \\
44276.41 \\
44323.00 \\
44515.01 \\
44529.428 \\
44689.913 \\
44891.22 \\
44919.302 \\
45018.23 \\
45091.022\end{array}$ & $\begin{array}{l}1.17 \\
1.18 \\
1.15 \\
1.205 \\
1.10 \\
1.16 \\
1.11 \\
1.04 \\
\\
1.16 \\
1.09 \\
1.125 \\
0.95 \\
1.19 \\
1.04\end{array}$ & $\begin{array}{l}4 f^{13}\left({ }^{2} \mathrm{~F}_{7 / 2}\right) 5 d 6 p\left({ }^{3} \mathrm{P}_{0}\right) \\
4 f^{13}\left({ }^{2} \mathrm{~F}_{7 / 2}\right) 5 d 6 p\left({ }^{3} \mathrm{P}_{2}\right)\end{array}$ & $\begin{array}{l}\left(3^{1 / 2}, 0\right) \\
\left(3^{1 / 2}, 2\right)\end{array}$ & $\begin{array}{l}3^{1 / 2} \\
3^{1 / 2} \\
5^{1 / 2} \\
5^{1 / 2} \\
4^{1 / 2}\end{array}$ & $\begin{array}{l}45131.67 \\
45149.63 \\
45714.11 \\
46262.14 \\
48547.98\end{array}$ & 1.15 & \\
\hline
\end{tabular}


TABLE 2. Energy levels of neutral thulium, odd parity, in units of $\mathrm{cm}^{-1}$. Ref. is given for levels previously published

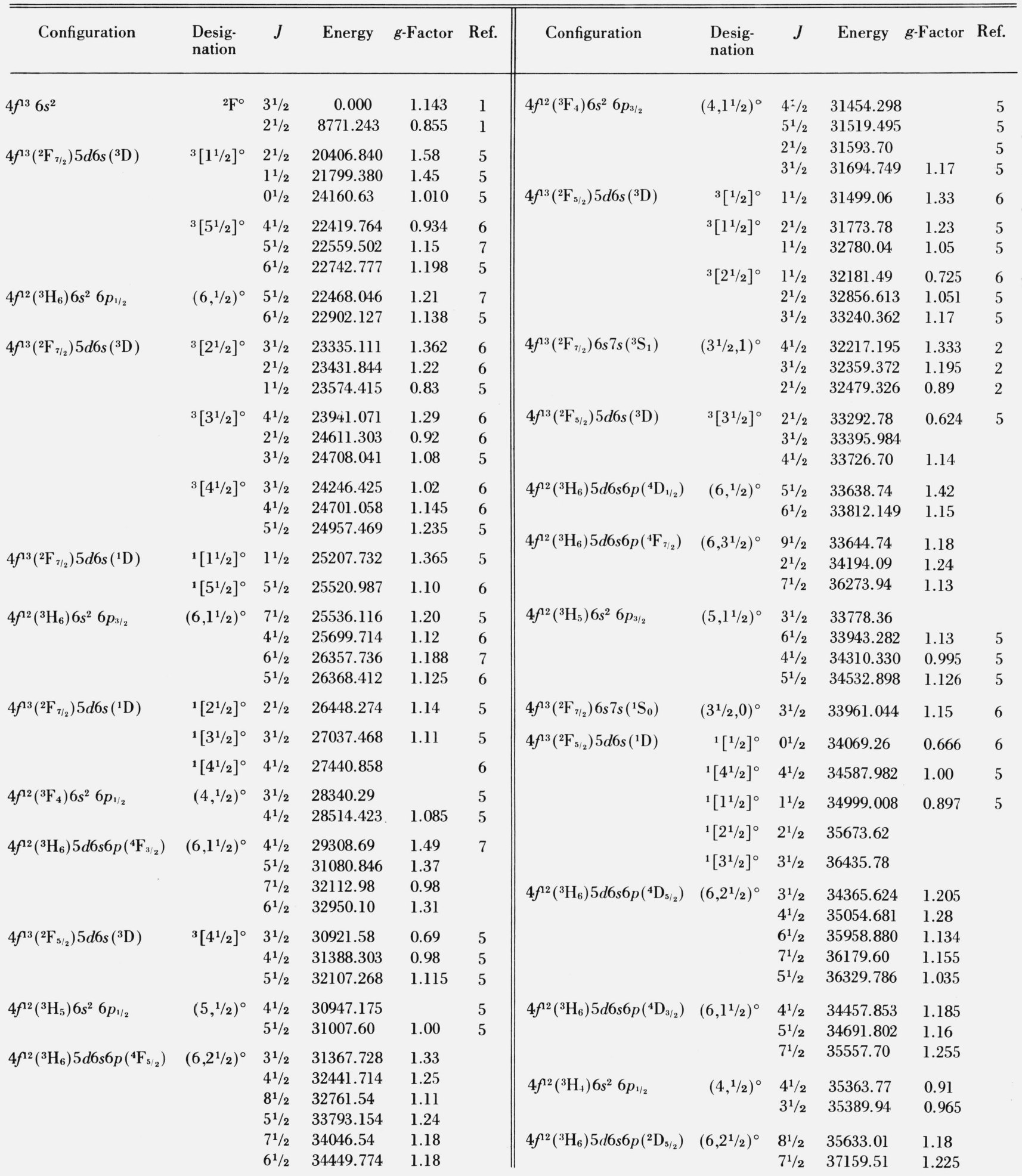


TABLE 2. Energy levels of neutral thulium, odd parity, in units of $\mathrm{cm}^{-1}$. Ref. is given for levels previously published-Continued

\begin{tabular}{|c|c|c|c|c|c|c|c|c|c|c|c|}
\hline Configuration & $\begin{array}{l}\text { Desig- } \\
\text { nation }\end{array}$ & $J$ & Energy & $g$-Factor & Ref. & Configuration & $\begin{array}{l}\text { Desig- } \\
\text { nation }\end{array}$ & $J$ & Energy & $g$-Factor & Ref. \\
\hline $4 f^{12} 5 d 6 s 6 p$ & & $\begin{array}{l}3^{1 / 2} \\
6^{1 / 2} \\
4^{1 / 2}\end{array}$ & $\begin{array}{l}35682.251 \\
36223.628 \\
36377.733\end{array}$ & $\begin{array}{l}1.222 \\
1.12 \\
1.21\end{array}$ & & $4 f^{3}\left({ }^{2} \mathrm{~F}_{7 / 2}\right) 6 s 6 d\left({ }^{3} \mathrm{D}\right)$ & ${ }^{3}\left[5^{1 / 2} / 2\right]^{\circ}$ & $\begin{array}{l}5^{1 / 2} \\
6^{1 / 2} \\
4^{1 / 2}\end{array}$ & $\begin{array}{l}39089.533 \\
39157.57 \\
39220.035\end{array}$ & $\begin{array}{l}1.09 \\
1.24 \\
1.12\end{array}$ & $\begin{array}{l}2 \\
6 \\
2\end{array}$ \\
\hline $4 f^{2}\left({ }^{3} \mathrm{H}_{6}\right) 5 d 6 s 6 p\left({ }^{2} \mathrm{D}_{3 / 2}\right)$ & $\left(6,1^{1 / 2}\right)^{\circ}$ & $7^{1 / 2}$ & 36653.924 & 1.13 & & & $3\left[2^{1 / 2}\right]^{\circ}$ & $\begin{array}{l}3^{1 / 2} \\
2^{1 / 2}\end{array}$ & $\begin{array}{l}39187.473 \\
39277.087\end{array}$ & $\begin{array}{l}1.336 \\
1.184\end{array}$ & 2 \\
\hline $4 f^{12} 5 d 6 s 6 p$ & & $\begin{array}{l}5^{1 / 2} \\
3^{1 / 2} \\
4^{1 / 2}\end{array}$ & $\begin{array}{l}36656.724 \\
36934.216 \\
37010.534\end{array}$ & $\begin{array}{l}1.16 \\
1.285 \\
1.11\end{array}$ & & $4 f^{12} 5 d 6 s 6 p$ & & $\begin{array}{l}1^{1 / 2} \\
4^{1 / 2}\end{array}$ & $\begin{array}{l}39419.90 \\
39244.584\end{array}$ & $\begin{array}{l}0.916 \\
1.05\end{array}$ & 2 \\
\hline $4 f^{2}\left({ }^{3} \mathrm{~F}_{3}\right) 6 s^{2} 6 p_{1 / 2}$ & $\left(3,{ }^{1} / 2\right)^{\circ}$ & $3^{1 / 2}$ & 37221.455 & & & $4 f^{33}\left({ }^{2} \mathrm{~F}_{7_{/ 2}}\right) 6 s 6 d\left({ }^{3} \mathrm{D}\right)$ & ${ }^{3}\left[3^{1 / 2}\right]^{\circ}$ & $\begin{array}{l}4^{1 / 2} \\
31 / 2\end{array}$ & $\begin{array}{l}39322.014 \\
39462.782\end{array}$ & $\begin{array}{l}1.153 \\
1.12\end{array}$ & \\
\hline $4 f^{12}\left({ }^{3} \mathrm{H}_{6}\right) 5 d 6 s 6 p\left({ }^{4} \mathrm{D}_{z / 2}\right)$ & $\left(6,3^{1 / 2}\right)^{\circ}$ & $\begin{array}{l}8^{1 / 2} \\
7^{1 / 2}\end{array}$ & $\begin{array}{l}37252.03 \\
37858.62\end{array}$ & $\begin{array}{l}1.20 \\
1.20\end{array}$ & & $4 f^{2} 5 d 6 s 6 p$ & & $\begin{array}{l}2^{1 / 2} \\
2^{1 / 2}\end{array}$ & $\begin{array}{l}39658.852 \\
39386.959\end{array}$ & $\begin{array}{l}0.96 \\
0.982\end{array}$ & \\
\hline $4 f^{12} 5 d 6 s 6 p$ & & $\begin{array}{l}5^{1 / 2} \\
6^{1 / 2}\end{array}$ & $\begin{array}{l}37276.778 \\
37365.13\end{array}$ & $\begin{array}{l}1.25 \\
1.20\end{array}$ & & & & $\begin{array}{l}3^{1 / 2} \\
6^{1 / 2}\end{array}$ & $\begin{array}{l}39413.672 \\
39434.151\end{array}$ & $\begin{array}{l}1.165 \\
1.15\end{array}$ & \\
\hline & & $\begin{array}{l}3^{1 / 2} \\
6^{1 / 2} \\
5^{1 / 2} \\
4^{1 / 2}\end{array}$ & $\begin{array}{l}37519.484 \\
37646.186 \\
37657.928 \\
37768.662\end{array}$ & $\begin{array}{l}1.09 \\
1.175 \\
1.01 \\
1.215\end{array}$ & & $4 f^{3}\left({ }^{2} \mathrm{~F}_{\tau_{1 / 2}}\right) 6 s 6 d\left({ }^{3} \mathrm{D}\right)$ & ${ }^{3}\left[4^{1 / 2}\right]^{\circ}$ & $\begin{array}{l}4^{1 / 2} \\
5^{1 / 2} \\
3^{1 / 2}\end{array}$ & $\begin{array}{l}39444.295 \\
39479.873 \\
39627.996\end{array}$ & $\begin{array}{l}1.13 \\
1.21 \\
1.04\end{array}$ & \\
\hline & & $\begin{array}{l}6^{1 / 2} \\
5^{1 / 2} \\
3^{1 / 2}\end{array}$ & $\begin{array}{l}38012.795 \\
38161.255 \\
38211.625\end{array}$ & $\begin{array}{l}1.09 \\
1.125 \\
1.175\end{array}$ & & $4 f^{2} 5 d 6 s 6 p$ & & $\begin{array}{l}3^{1 / 2} \\
4^{1 / 2} \\
5^{1 / 2}\end{array}$ & $\begin{array}{l}39480.272 \\
39488.236 \\
39563.003\end{array}$ & $\begin{array}{l}1.17 \\
1.095 \\
1.11\end{array}$ & \\
\hline $4 f^{12}\left({ }^{3} \mathrm{H}_{4}\right) 6 s^{2} 6 p_{3 / 2}$ & $\left(4,1^{1 / 2}\right)^{\circ}$ & $\begin{array}{l}5^{1 / 2} \\
31 / 2 \\
4^{1 / 2}\end{array}$ & $\begin{array}{l}38347.875 \\
38664.184 \\
38685.555\end{array}$ & $\begin{array}{l}1.07 \\
1.042 \\
1.043\end{array}$ & & $4 f^{12}\left({ }^{3} \mathrm{~F}_{3}\right) 6 s^{2} 6 p_{3 / 2}$ & $\left(3,1^{1 / 2}\right)^{\circ}$ & $\begin{array}{l}3^{1 / 2} \\
4^{1 / 2}\end{array}$ & $\begin{array}{l}39709.038 \\
40061.324 \\
40208.499\end{array}$ & $\begin{array}{l}1.17 \\
1.13 \\
1.18\end{array}$ & \\
\hline $4 f^{12} 5 d 6 s 6 p$ & & $\begin{array}{l}4^{1 / 2} \\
3^{1 / 2}\end{array}$ & $\begin{array}{l}38384.926 \\
38482.584\end{array}$ & $\begin{array}{l}1.134 \\
1.25\end{array}$ & & $4 f^{12} 5 d 6 s 6 p$ & & $\begin{array}{l}6^{1 / 2} \\
4^{1 / 2}\end{array}$ & $\begin{array}{l}40113.75 \\
40322.536\end{array}$ & 1.115 & \\
\hline $4 f^{2}\left({ }^{3} \mathrm{H}_{6}\right) 5 d 6 s 6 p\left({ }^{4} \mathbf{F}_{9 / 2}\right)$ & $\left(6,4^{1 / 2}\right)^{\circ}$ & $7^{1 / 2}$ & 38485.85 & 1.145 & & & & $\begin{array}{l}2^{1 / 2} \\
6^{1 / 2}\end{array}$ & $\begin{array}{l}40347.267 \\
40376.20\end{array}$ & 1.14 & \\
\hline $4 f^{2} 5 d 6 s 6 p$ & & $\begin{array}{l}4^{1 / 2} \\
5^{1 / 2} \\
6^{1 / 2} \\
2^{1 / 2}\end{array}$ & $\begin{array}{l}38499.016 \\
38529.686 \\
38588.46 \\
38751.812\end{array}$ & $\begin{array}{l}1.064 \\
1.17 \\
1.212 \\
1.215\end{array}$ & & & & $\begin{array}{l}4^{1 / 2} \\
3^{1 / 2} \\
3^{1 / 2} \\
5^{1 / 2} \\
3^{1 / 2}\end{array}$ & $\begin{array}{l}40517.661 \\
40533.825 \\
40596.302 \\
40663.129 \\
40763.892\end{array}$ & $\begin{array}{l}1.153 \\
1.24 \\
1.305 \\
\\
1.178\end{array}$ & 2 \\
\hline $4 f^{13}\left({ }^{2} \mathrm{~F}_{7_{/ 2}}\right) 6 s 6 d\left({ }^{3} \mathrm{D}\right)$ & ${ }^{3}\left[1^{1 / 2}\right]^{\circ}$ & $\begin{array}{l}2^{1 / 2} \\
1^{1 / 2} \\
0^{1 / 2}\end{array}$ & $\begin{array}{l}38861.937 \\
39602.902 \\
39737.58\end{array}$ & $\begin{array}{l}1.565 \\
1.30 \\
0.81\end{array}$ & $\begin{array}{l}7 \\
7\end{array}$ & & & $\begin{array}{l}6^{1 / 2} \\
4^{1 / 2} \\
2^{1 / 2}\end{array}$ & $\begin{array}{l}40780.904 \\
40855.580 \\
40938.61\end{array}$ & $\begin{array}{l}1.22 \\
1.08 \\
1.20\end{array}$ & \\
\hline $4 f^{12} 5 d 6 s 6 p$ & & $\begin{array}{l}5^{1 / 2} \\
6^{1 / 2}\end{array}$ & $\begin{array}{l}38877.211 \\
38915.90\end{array}$ & $\begin{array}{l}1.065 \\
1.22\end{array}$ & & $4 f^{13}\left({ }^{2} \mathrm{~F}_{5 / 2}\right) 6 s 7 s\left({ }^{3} \mathrm{~S}_{1}\right)$ & $\left(2^{1 / 2}, 1\right)^{\circ}$ & $\begin{array}{l}1^{1 / 2} \\
2^{1 / 2} \\
3^{1 / 2}\end{array}$ & $\begin{array}{l}40989.73 \\
41074.399 \\
41189.162\end{array}$ & $\begin{array}{l}0.40 \\
0.99 \\
1.19\end{array}$ & $\begin{array}{l}6 \\
6 \\
7\end{array}$ \\
\hline $4 f^{13}\left({ }^{2} \mathrm{~F}_{7_{/ 2}}\right) 6 s 6 d\left({ }^{1} \mathrm{D}\right)$ & $\begin{array}{l}{ }^{1}\left[1^{1 / 2}\right]^{\circ} \\
{ }^{1}\left[5^{1 / 2} / 2\right]^{\circ}\end{array}$ & $\begin{array}{l}1^{1 / 2} \\
51 / 2\end{array}$ & $\begin{array}{l}39061.819 \\
39362.651\end{array}$ & $\begin{array}{l}1.34 \\
1.13\end{array}$ & 2 & $4 f^{12} 5 d 6 s 6 p$ & & $\begin{array}{l}3^{1 / 2} \\
5^{1 / 2}\end{array}$ & $\begin{array}{l}41012.387 \\
41081.384\end{array}$ & $\begin{array}{l}1.15 \\
1.295\end{array}$ & \\
\hline & $\begin{array}{c}{ }^{1}\left[2^{1 / 2}\right]^{\circ} \\
{ }^{1}\left[3^{1 / 2}\right]^{\circ} \\
{ }^{1}\left[4^{1 / 2}\right]^{\circ}\end{array}$ & $\begin{array}{l}2^{1 / 2} \\
3^{1 / 2} \\
4^{1 / 2}\end{array}$ & $\begin{array}{l}39470.489 \\
39542.165 \\
39741.913\end{array}$ & $\begin{array}{l}1.04 \\
1.06 \\
1.112\end{array}$ & 2 & $4 f^{13}\left({ }^{2} \mathrm{~F}_{7 / 2}\right) 6 s 8 s\left({ }^{3} \mathrm{~S}_{1}\right)$ & $\left(3^{1 / 2}, 1\right)^{\circ}$ & $\begin{array}{l}4^{1 / 2} \\
3^{1 / 2} \\
2^{1 / 2}\end{array}$ & $\begin{array}{l}41078.537 \\
41211.943 \\
41325.87\end{array}$ & $\begin{array}{l}1.32 \\
1.18 \\
0.90\end{array}$ & $\begin{array}{l}7 \\
6 \\
7\end{array}$ \\
\hline
\end{tabular}


TABLE 2. Energy levels of neutral thulium, odd parity, in units of $\mathrm{cm}^{-1}$. Ref. is given for levels previously published-Continued

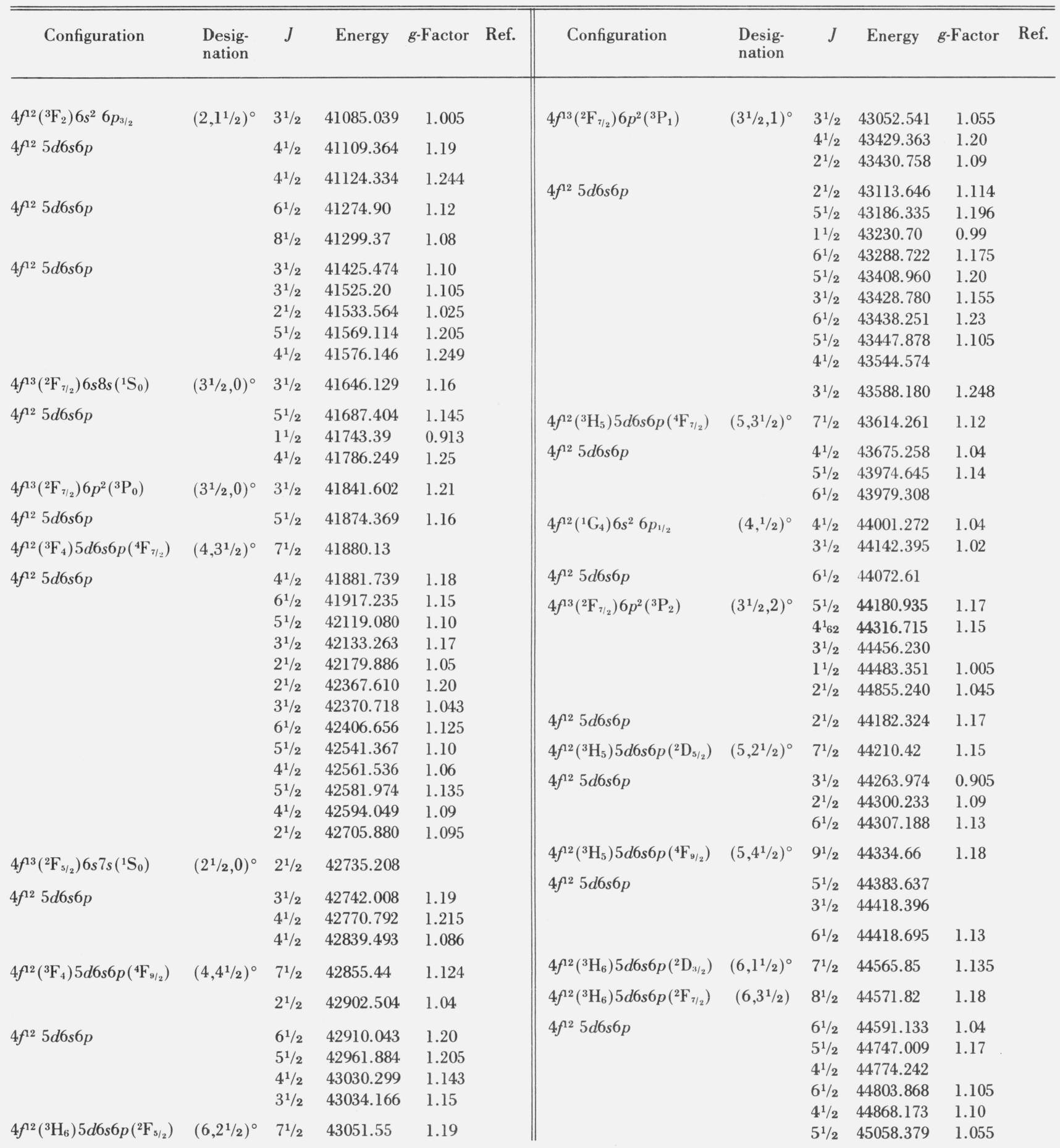


TABLE 2. Energy levels of neutral thulium, odd parity, in units of $\mathrm{cm}^{-1}$. Ref. is given for levels previously published-Continued

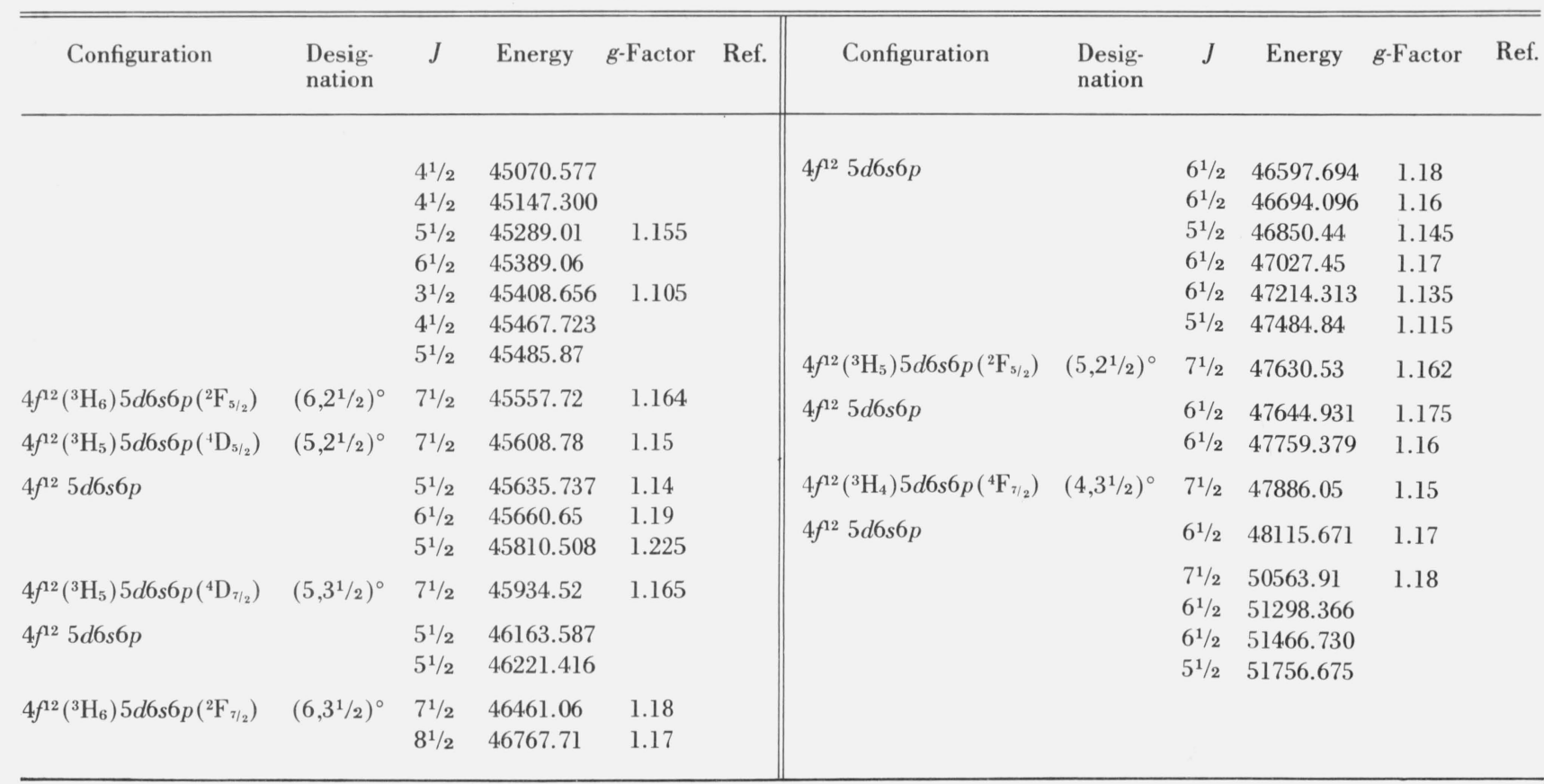

\section{Ionization Energy}

An accurate value for the ionization energy of neutral thulium was derived by Camus [23] from his observations of the $4 f^{13}\left({ }^{2} \mathrm{~F}_{7 / 2}\right) 6 s n p$ series in absorption for $17 \leqslant n \leqslant 48$. He obtained the value $49879.5 \pm 0.5$ $\mathrm{cm}^{-1}$. This supports the value obtained from the $4 f^{13}\left({ }^{2} \mathrm{~F}_{7 / 2}\right) 6 s n s$ series $(7 \leqslant n \leqslant 8)$. From the data reported in table 2 we have:

$$
\text { center of gravity } \begin{aligned}
4 f^{13}\left({ }^{2} \mathrm{~F}_{7 / 2}\right) 6 s 7 s & =32737.9 \mathrm{~cm}^{-1} \\
6 s 8 s & =41300.2 \mathrm{~cm}^{-1} .
\end{aligned}
$$

Assuming a change in quantum defect of $1.031 \pm$ 0.005 deduced from the corresponding configurations of $\mathrm{Ba} \mathrm{I},[25]$ we obtain a series limit of $49994.4 \pm 20$ $\mathrm{cm}^{-1}$. Since the limit occurs at $103.7 \mathrm{~cm}^{-1}$ above the ground state of Tm II, the ionization energy equals $49891 \pm 20 \mathrm{~cm}^{-1}$. We note that this result confirms the predicted value by Reader and Sugar [26] of $49900 \pm 160 \mathrm{~cm}^{-1}$, based on interpolation of the $6 \mathrm{sns}$ series for all the rare earths.

We wish to thank L. J. Radziemski for processing our line list with his computer program (see ref. [24]) to determine the best values for the energy levels.

\section{References}

[1] Meggers, W. F., Rev. Mod. Phys. 14,96 (1942).

[2] Blaise, J., and Vetter, R., Compt. Rend. 256,630 (1963).
[3] Bordarier, Y., Vetter, R., and Blaise, J., Jour. de Physique 24, 1107 (1963).

[4] Sugar, J., J. Opt. Soc. Am. 60, 454 (1970).

[5] Camus, P., Guelachvilli, G., and Verges, J., Spectr. Act. 24B, 373 (1969).

[6] Blaise, J., and Camus, P., Compt. Rend. 260, 4693 (1965).

[7] Camus, P., and Blaise, J., Compt. Rend. 261, 4359 (1965).

[8] Camus, P., Jour. de Phys. 27,717 (1966).

[9] Camus, P., Jour. de Phys. 33, 203 (1972).

[10] Corliss, C. H., Bozman, W. R., and Westfall, F. O., J. Opt. Soc. Am. 43, 398 (1953).

[11] Tomkins, F. S., and Fred, M., J. Opt. Soc. Am. 47, 1087 (1957).

[12] Sugar, J., J. Opt. Soc. Am. 53, 831 (1963).

[13] Sugar, J., J. Opt. Soc. Am. 55, 33 (1965).

[14] Tomkins, F. S., and Fred, M., Spectrochim. Acta 6, 139 (1954).

[15] Tomkins, F. S., and Fred, M., Appl. Opt. 2, 715 (1963).

[16] Reader. J., Meissner, K. W., and Andrew, K. L., J. Opt. Soc. Am. 50,221 (1960)

[17] Van Veld, R. D., and Meissner, K. W., J. Opt. Soc. Am. 46 , 598 (1956).

[18] Meggers, W. F., and Humphreys, C. J., J. Res. Nat. Bur. Stand. (U.S.), 18, 543 (1937) RP992.

[19] Crosswhite, H. M., Johns Hopkins Spectroscopic Rept. 13 (1958).

[20] Meggers, W. F., and Stanley, R. W., J. Res. Nat. Bur. Stand. (U.S.), 69A (Phys. and Chem.), No. 2, 109-118 (Mar.-Apr. (1965)).

[21] Sugar, J., J. Res. Nat. Bur. Stand. (U.S.), 66A (Phys. and Chem.), No. 4, 321-324. (July-Aug. 1962).

[22] Brewer, L., J. Opt. Soc. Am. 61,1101 (1971).

[23] Camus, P., Thesis, Orsay (1971).

[24] Radziemski, L. J., Fisher, K. J., Steinhaus, D. W., and Goldman, A. S., Comp. Phys. Comm. 3,9 (1972).

[25] Russell H. N., and Moore, C. E., J. Res. Nat. Bur. Stand. (U.S.), 55, 299 (1955) RP2633.

[26] Reader, J., and Sugar, J., J. Opt. Soc. Am. 56, 1189 (1966).

(Paper 77A1-752) 
APPENDIX - Spectral Lines of Neutral Thulium

\begin{tabular}{|c|c|c|c|c|c|c|c|c|c|}
\hline \multirow{2}{*}{$\begin{array}{c}\text { Wave- } \\
\text { length } \\
\text { in air } \\
\AA\end{array}$} & \multirow[t]{2}{*}{ Intensity } & \multirow{2}{*}{$\begin{array}{c}\text { Wave- } \\
\text { number } \\
\mathrm{cm}^{-1}\end{array}$} & \multirow[t]{2}{*}{ Classification } & \multirow{2}{*}{\begin{tabular}{ccc}
\multicolumn{3}{c}{ Zeeman effect } \\
type $\quad \mathrm{f} \quad \mathrm{Je}$
\end{tabular}} & \multirow{2}{*}{$\begin{array}{c}\text { Wave- } \\
\text { length } \\
\text { in air } \\
\AA\end{array}$} & \multirow[t]{2}{*}{ Intensity } & \multirow{2}{*}{$\begin{array}{c}\text { Wave- } \\
\text { number } \\
\text { cm }^{-1}\end{array}$} & Classification & Zeeman effect \\
\hline & & & & & & & & & type $\mathrm{f} \quad \mathrm{Je}$ \\
\hline 11749.50 & 5 & 8508.67 & & & 10827.20 & 30 & 9233.47 & & \\
\hline 11662.00 & $5 h$ & 8572.51 & & & 10812.73 & $5 h$ & 9245.83 & $33489_{3 / 2}-42735_{5 / 2}^{\circ}$ & \\
\hline 11650.30 & 4 & 8581.12 & & & 10803.30 & 1 & 9253.90 & $25745_{s_{/ 2}}-34999^{\circ}{ }_{3 / 2}^{\circ}$ & \\
\hline 11649.00 & $5 h$ & 8582.08 & $33961^{\circ}{ }_{\eta / 2}-42543_{7 / 2}$ & & 10797.36 & 3 & 9258.99 & & \\
\hline 11544.00 & 5 & 8660.14 & & & 10786.90 & 2 & 9267.97 & & \\
\hline 11540.00 & 5 & 8663.14 & $22791_{7 / 2}-31454_{9 / 2}^{\circ}$ & & 10785.90 & 1 & 9268.82 & & \\
\hline 11538.90 & 3 & 8663.97 & $22929_{s / 2}-31593_{s / 2}^{\circ / 2}$ & & 10784.67 & 4 & 9269.88 & $21737_{9 / 2}-31007^{\circ}{ }_{11 / 2}$ & \\
\hline 11522.70 & 1 & 8676.15 & & & 10767.31 & 80 & 9284.83 & $17752_{3 / 2}-27037^{\circ}{ }_{7 / 2}^{2}$ & \\
\hline 11515.30 & 50 & 8681.72 & $25130_{13 / 2}-33812^{\circ}{ }_{13 / 2}$ & & 10763.70 & 3 & 9287.94 & & \\
\hline 11496.90 & 10 & 8695.62 & $17752_{s / 2}-26448^{\circ / 2}$ & & 10757.30 & 2 & 9293.47 & $30915_{9 / 2}-40208_{9 / 2}^{\circ}$ & \\
\hline 11452.30 & 1 & 8729.48 & & & 10749.44 & 5 & 9300.26 & $13119_{9 / 2}-22419_{9 / 2}^{\circ}$ & \\
\hline 11434.90 & 200 & 8742.76 & $16957_{\eta_{/ 2}}-25699_{9 / 2}^{\circ}$ & & 10745.85 & 4 & 9303.37 & $39244_{9 / 2}^{\circ / 2}-48547_{9 / 2}^{1 / 2}$ & \\
\hline 11423.20 & 1 & 8751.72 & & & 10738.30 & 1 & 9309.91 & & \\
\hline 11419.20 & 10 & 8754.78 & $17613_{g^{2} / 2}-26368^{\circ}{ }_{11 / 2}$ & & 10727.00 & 5 & 9319.72 & & \\
\hline 11405.90 & 7 & 8764.99 & $22929_{\mathrm{s} / 2}-31694_{7_{/ 2}}^{\circ}$ & & 10701.75 & 1 & 9341.71 & & \\
\hline 11397.80 & 1 & 8771.22 & & & 10700.30 & 8 & 9342.97 & $25656_{s_{/ 2}}-34999^{\circ}{ }_{3 / 2}$ & \\
\hline 11395.80 & 1 & 8772.76 & $28448_{s / 2}-37221^{\circ}{ }_{7 / 2}$ & & 10694.03 & 2000 & 9348.45 & $13119_{9 / 2}-22468^{\circ}{ }_{11 / 2}$ & \\
\hline 11387.40 & 4 & 8779.23 & & & 10680.43 & 40 & 9360.35 & $24418_{s / 2}-33778_{z_{/ 2}}^{\circ}$ & \\
\hline 11357.30 & 3 & 8802.50 & $22791_{7 / 2}-31593^{\circ}{ }_{s / 2}$ & & 10673.60 & 1 & 9366.34 & $20.700 / 2$ & \\
\hline & & & $27377_{13 / 2}-36179^{\circ}{ }_{15 / 2}^{\prime 2}$ & & 10672.70 & 6 & 9367.13 & $23873_{7 / 2}-33240^{\circ}{ }^{\circ}$ & \\
\hline 11344.00 & 30 & 8812.82 & $25130_{13 / 2}-33943^{\circ}{ }_{13 / 2}$ & & 10670.80 & 1 & 9368.80 & & \\
\hline & & & $24137_{13 / 2}-32950^{\circ}{ }_{13 / 2}^{\circ}$ & & 10669.80 & 2 & 9369.68 & $15587_{11 / 2}-24957^{\circ}{ }_{11 / 2}$ & \\
\hline 11332.50 & 5 & 8821.76 & $25488_{11 / 2}-34310^{\circ}$ & & 10661.10 & 4 & 9377.33 & $24246_{\gamma_{/ 2}}^{\circ}-33623_{\gamma_{2}}$ & \\
\hline 11285.80 & 3 & 8858.27 & & & 10653.90 & 1 & 9383.66 & & \\
\hline 11284.10 & 4 & 8859.60 & & & 10631.55 & $20 d$ & 9403.39 & & \\
\hline 11269.90 & 6 & 8870.76 & $25717_{\tau_{/ 2}}-34587^{\circ}{ }_{9 / 2}$ & & 10613.80 & 2 & 9419.12 & & \\
\hline 11268.20 & 8 & 8872.10 & $26126_{5 / 2}-34999^{\circ}{ }_{3 / 2}^{\circ / 2}$ & & 10606.10 & 8 & 9425.95 & $22791_{7 / 2}-32217^{\circ}{ }_{9 / 2}$ & \\
\hline 11261.40 & 2 & 8877.46 & $25207^{\circ}{ }_{3 / 2}^{\circ}-34085_{s_{/ 2}}$ & & 10603.50 & 1 & 9428.27 & & \\
\hline 11229.20 & 100 & 8902.92 & $17454_{13 / 2}-26357^{\circ}{ }_{13 / 2}^{1 / 2}$ & & 10602.00 & 7 & 9429.60 & $22929_{s / 2}-32359^{\circ}{ }_{7 / 2}$ & \\
\hline 11228.40 & 10 & 8903.55 & $22791_{7 / 2}-31694_{7 / 2}^{\circ / 2}$ & & 10590.45 & 2000 & 9439.88 & $13119_{9 / 2}^{0 / 2}-22559^{\circ}{ }_{11 / 2}^{1 / 2}$ & \\
\hline 11215.70 & 200 & 8913.63 & $17454_{13 / 2}-26368^{\circ}{ }_{11 / 2}$ & & 10585.30 & 6 & 9444.48 & $24348_{9 / 2}-33793^{\circ}{ }_{11 / 2}$ & \\
\hline 11207.00 & 2 & 8920.55 & $35261_{s_{/ 2}}-44182^{\circ}{ }_{s / 2}^{\circ}$ & & 10569.68 & 2 & 9458.43 & $39089^{\circ}{ }_{11 / 2}^{2}-48547_{9 / 2}$ & \\
\hline 11202.40 & 2 & 8924.22 & $26439_{\eta_{/ / 2}}-35363^{\circ}{ }_{9_{/ 2}}$ & & 10565.00 & 10 & 9462.62 & & \\
\hline 11175.30 & 1 & 8945.86 & & & 10539.95 & 20 & 9485.11 & & \\
\hline 11172.50 & 2 & 8948.10 & & & 10533.14 & 6 & 9491.24 & $16957_{\tau_{/ 2}}-26448^{\circ}{ }_{s / 2}$ & \\
\hline 11165.00 & 70 & 8954.11 & & & 10531.20 & 3 & 9492.99 & & \\
\hline 11164.30 & 20 & 8954.67 & $31694^{\circ}{ }_{7 / 2}-40649_{\tau_{1 / 2}}$ & & 10530.25 & 3 & 9493.85 & & \\
\hline 11160.80 & 5 & 8957.48 & $16742_{\eta_{/ 2}}^{/ 2}-25699^{\circ}{ }_{9 / 2}^{1 / 2}$ & & 10520.27 & 20 & 9502.86 & $18837_{9 / 2}-28340^{\circ}{ }_{7 / 2}$ & \\
\hline 11154.30 & 1 & 8962.70 & & & 10499.11 & 200 & 9522.01 & $21997_{11 / 2}-31519^{\circ}{ }_{11 / 2}$ & \\
\hline 11153.30 & 1 & 8963.50 & & & 10496.90 & 7 & 9524.01 & $18990_{11 / 2}^{/ 2}-28514_{9 / 2}^{\circ}$ & \\
\hline 11144.40 & 2 & 8970.66 & & & 10476.05 & 2000 & 9542.97 & $24418_{5 / 2}-33961_{7 / 2}^{\circ}$ & \\
\hline 11135.30 & 40 & 8977.99 & $24418_{s / 2}-33396^{\circ}$ & & 10471.96 & 5 & 9546.70 & $26126_{\mathrm{s} / 2}-35673_{\mathrm{s} / 2}^{\circ}$ & \\
\hline 11131.10 & 2 & 8981.38 & $8771_{\mathrm{s} / 2}^{\circ}-17752_{\mathrm{s}_{2}}$ & & " & & & $26889_{9 / 2}-36435^{\circ}{ }_{7 / 2}$ & \\
\hline 11128.55 & $3 h$ & 8983.44 & $23873_{\eta_{/ 2}}-32856^{\circ}{ }_{\mathrm{s} / 2}^{2}$ & & 10454.60 & 10 & 9562.55 & & \\
\hline 11104.25 & 2 & 9003.10 & & & 10448.46 & 2 & 9568.17 & $22791_{7 / 2}-32359^{\circ}{ }_{7 / 2}$ & \\
\hline 11100.90 & 2 & 9005.81 & $39542_{7_{1 / 2}}^{\circ}-48547_{9_{/ 2}}$ & & 10441.30 & 1 & 9574.73 & & \\
\hline 11095.60 & 20 & 9010.11 & $21997_{11 / 2}-31007^{\circ}{ }_{11 / 2}^{\circ}$ & & 10400.48 & 2000 & 9612.31 & $24348_{9 / 2}-33961^{\circ}{ }_{7 / 2}$ & \\
\hline 11071.28 & 2 & 9029.91 & & & 10381.35 & 10 & 9630.02 & $21737_{9 / 2}-31367^{\circ}{ }_{7_{/ 2}}^{T / 2}$ & \\
\hline 11069.71 & 2 & 9031.19 & $29316_{9 / 2}-38347^{\circ}{ }_{11 / 2}$ & & 10365.44 & 10 & 9644.80 & $25745_{s / 2}-35389^{\circ}{ }_{7 / 2}$ & \\
\hline 11053.60 & 20 & 9044.35 & $25488_{11 / 2}-34583^{\circ}{ }_{11 / 2}^{1 / 2}$ & & 10359.19 & 4 & 9650.62 & $21737_{9 / 2}-31388_{9 / 2}^{\circ}$ & \\
\hline 11039.95 & 20 & 9055.53 & & & 10353.10 & 1 & 9656.30 & & \\
\hline 11029.96 & 5 & 9063.73 & & & 10348.50 & 40 & 9660.59 & $18853_{11 / 2}-28514_{9 / 2}^{\circ}$ & \\
\hline 11011.15 & 2000 & 9079.22 & $16456_{17 / 2}-25536^{\circ}{ }^{\circ}{ }^{15 / 2}$ & & 10343.88 & 200 & 9664.90 & & \\
\hline 11006.11 & 2 & 9083.37 & $21997_{11 / 2}-31080^{\circ}{ }_{11 / 2}$ & & 10341.75 & 1 & 9666.89 & & \\
\hline 10986.66 & 10 & 9099.46 & $25488_{11 / 2}-34587_{9 / 2}^{\circ}$ & & 10334.53 & 2 & 9673.65 & $31519^{\circ}{ }_{11 / 2}-41193_{13 / 2}$ & \\
\hline 10980.10 & 100 & 9104.89 & $17343_{7_{7 / 2}}-26448_{s / 2}^{\circ}$ & & 10319.12 & 6 & 9688.09 & $22791_{7 / 2}-32479^{\circ}{ }^{\circ} / 2$ & \\
\hline 10972.35 & 15 & 9111.32 & $30302_{s / 2}-39413^{\circ}$ & & 10316.32 & 4 & 9690.72 & $30972_{11 / 2}-40663^{\circ}{ }_{11 / 2}^{\circ}$ & \\
\hline 10963.91 & 3 & 9118.34 & & & 10312.75 & 200 & 9694.08 & $17343_{\tau_{/ 2}}-27037^{\circ}{ }_{7 / 2}^{1 / 2}$ & \\
\hline 10953.48 & 10 & 9127.02 & & & 10300.07 & 3 & 9706.01 & $16742_{7 / 2}-26448_{5 / 2}^{\circ}$ & \\
\hline 10917.44 & 1 & 9157.15 & & & 10288.86 & 80 & 9716.59 & $21737_{9 / 2}-31454_{9 / 2}^{\circ / 2}$ & \\
\hline 10907.07 & 3 & 9165.85 & $29316_{9 / 2}-38482^{\circ}{ }_{7 / 2}$ & & 10282.10 & 1 & 9722.98 & & \\
\hline 10889.90 & 4 & 9180.31 & & & 10269.68 & 2 & 9734.73 & & \\
\hline 10869.60 & 30 & 9197.45 & & & 10229.61 & $20 h$ & 9772.87 & $29316_{9 / 2}-39089^{\circ}{ }_{11 / 2}$ & \\
\hline 10862.75 & 4 & 9203.25 & $25488_{11 / 2}-34691^{\circ}{ }_{11 / 2}$ & & 10225.17 & $3 h$ & 9777.11 & $32928_{3 / 2}-42705^{\circ}{ }_{5 / 2}^{\circ / 2}$ & \\
\hline 10852.52 & 10 & 9211.93 & & & 10220.30 & 100 & 9781.77 & $21737_{9 / 2}-31519^{\circ}{ }_{11 / 2}$ & \\
\hline 10851.34 & 40 & 9212.93 & $28555_{\eta_{/ 2}}-37768_{9 / 2}^{\circ}$ & & 10216.05 & 1 & 9785.84 & & \\
\hline 10849.05 & 15 & 9214.87 & & & 10197.54 & 2 & 9803.60 & & \\
\hline 10840.50 & $2 h$ & 9222.14 & & & 10194.60 & 30 & 9806.43 & $32928_{3 / 2}-42735_{s_{/ 2}}^{\circ}$ & \\
\hline 10838.90 & 1 & 9223.50 & & & 10182.06 & 20 & 9818.50 & $25207^{\circ / 2}-35026_{5 / 2}$ & \\
\hline 10837.30 & $2 h$ & 9224.86 & & & 10173.95 & 4 & 9826.33 & $21120_{z^{\prime} / 2}-30947_{9 / 2}^{\circ}$ & \\
\hline
\end{tabular}


APPENDix - Spectral Lines of Neutral Thulium-Continued

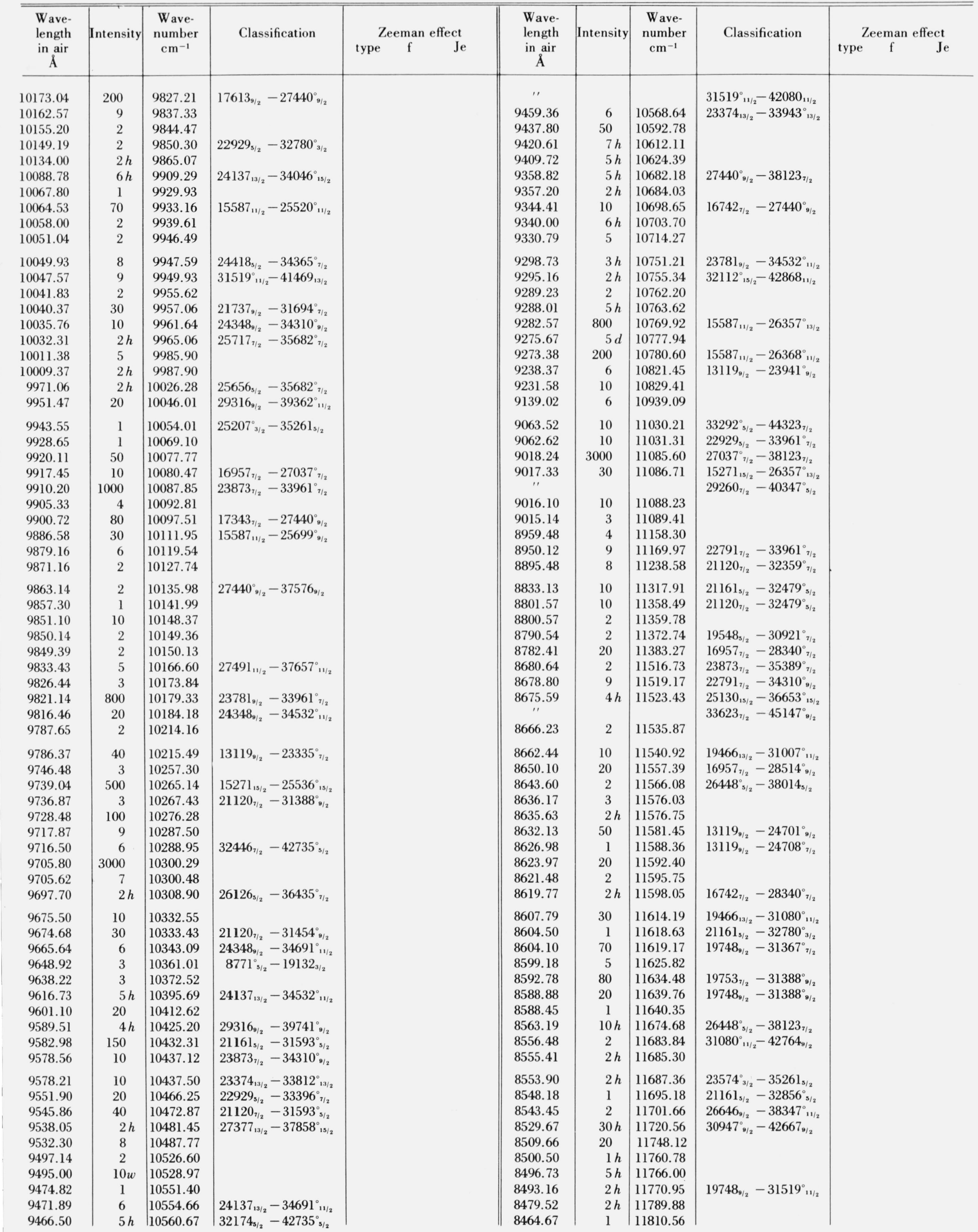


APPENDIX - Spectral Lines of Neutral Thulium-Continued

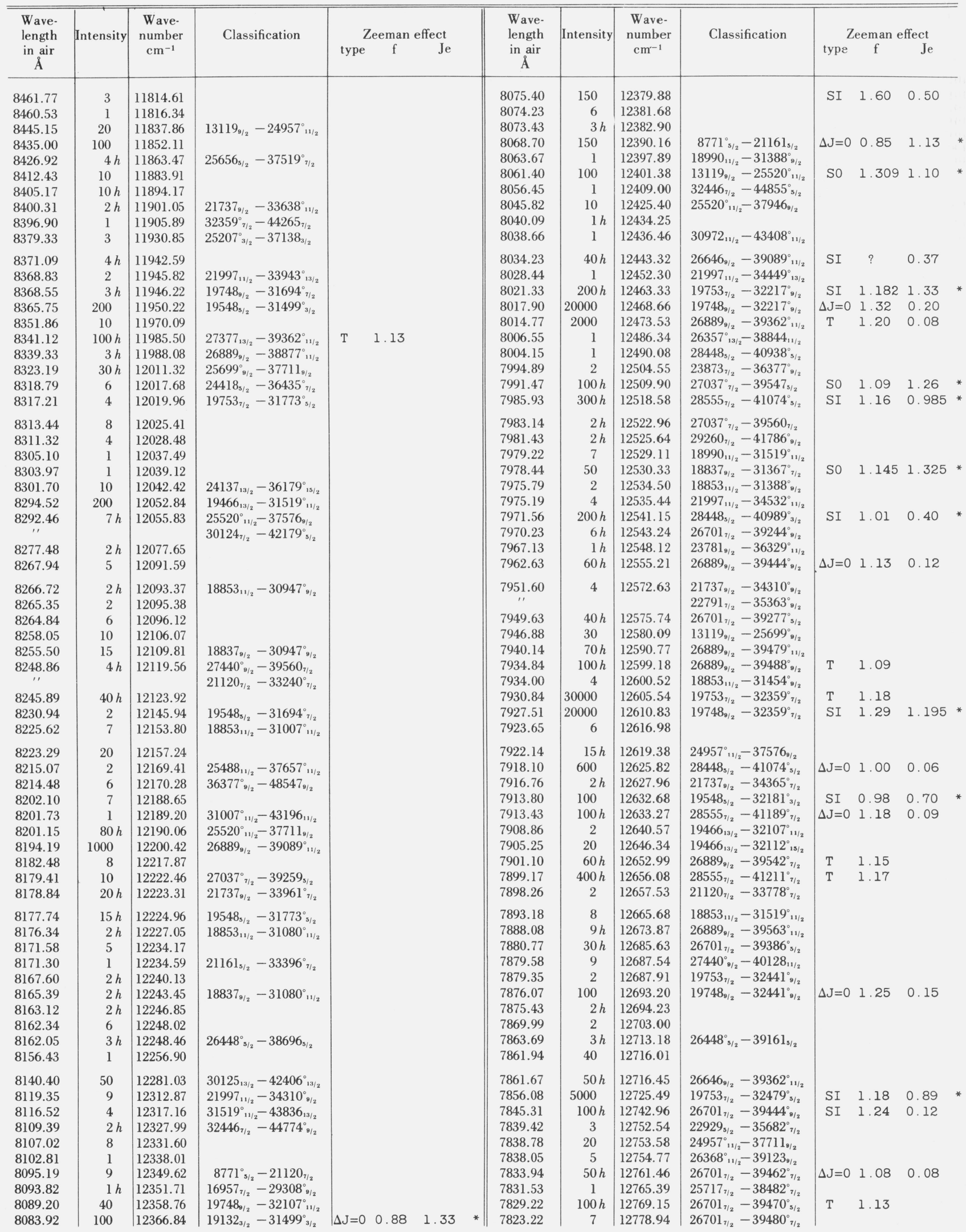


APPENDiX - Spectral Lines of Neutral Thulium-Continued

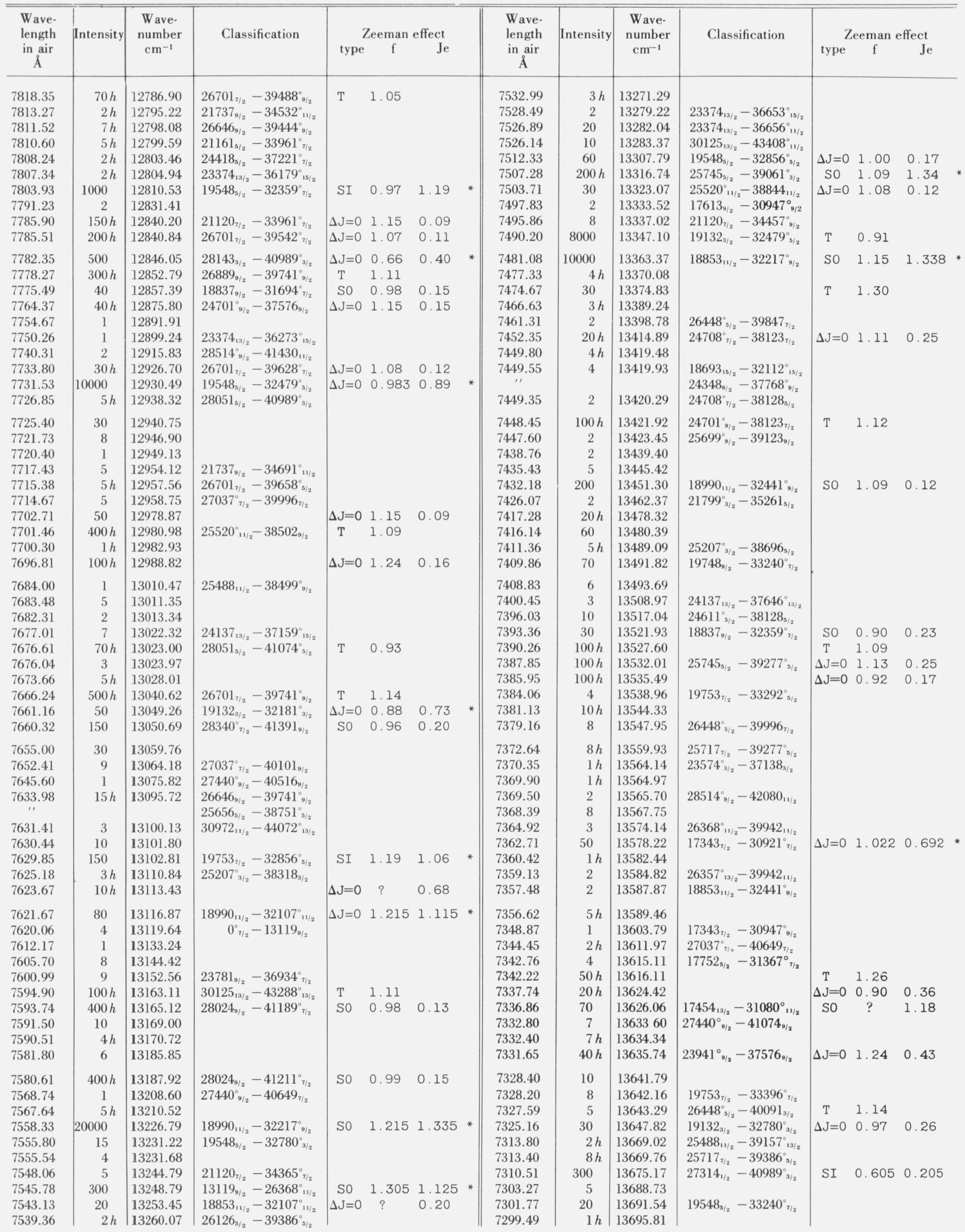


APPENDIX - Spectral Lines of Neutral Thulium-Continued

\begin{tabular}{|c|c|c|c|c|c|c|c|c|c|c|c|c|c|c|c|}
\hline \multirow{2}{*}{$\begin{array}{c}\text { Wave- } \\
\text { length } \\
\text { in air } \\
\AA\end{array}$} & \multirow[t]{2}{*}{ Intensity } & \multirow{2}{*}{$\begin{array}{c}\text { Wave- } \\
\text { number } \\
\mathrm{cm}^{-1}\end{array}$} & \multirow[t]{2}{*}{ Classification } & \multirow{2}{*}{\multicolumn{4}{|c|}{ e $\quad \mathrm{f} \quad \mathrm{Je}$}} & \multirow{2}{*}{$\begin{array}{c}\text { Wave- } \\
\text { length } \\
\text { in air } \\
\AA\end{array}$} & Intensity & $\begin{array}{l}\text { Wave- } \\
\text { number }\end{array}$ & Classification & & leeman $\mathbf{e}$ & effect & \\
\hline & & & & & & & & & & $\mathrm{cm}^{-1}$ & & type & f & $\mathrm{Je}$ & \\
\hline $\begin{array}{c}7293.67 \\
\text {," }\end{array}$ & 6 & 13706.74 & $\begin{array}{l}23431_{5 / 2}^{\circ}-37138_{3 / 2} \\
31440_{9 / 2}-45147_{9 / 2}^{\circ}\end{array}$ & & & & & $\begin{array}{l}7099.63 \\
7095.21\end{array}$ & $\begin{array}{l}10 h \\
50\end{array}$ & $\begin{array}{l}14081.36 \\
14090.13\end{array}$ & $\begin{array}{l}28514_{9 / 2}^{\circ}-42595_{11 / 2} \\
34457_{9 / 2}^{\circ}-48547_{9 / 2}\end{array}$ & $\Delta \mathrm{J}=0$ & 1.085 & 0.21 & \\
\hline 7293.43 & $5 h$ & 13707.19 & $26889_{9 / 2}-40596^{\circ}{ }_{7 / 2}$ & & & & & 7092.06 & 5 & 14096.39 & $18853_{11 / 2}-32950^{\circ}{ }_{13 / 2}$ & & & & \\
\hline 7291.81 & 4 & 13710.24 & $27440_{9 / 2}^{\circ}-41151_{9 / 2}$ & & & & & 7083.40 & $5 h$ & 14113.62 & $27037^{\circ}{ }^{2}-41151_{9 / 2}$ & & & & \\
\hline 7287.86 & $8 h$ & 13717.67 & $25745_{5 / 2}^{\circ / 2}-39462^{\circ}{ }_{7 / 2}$ & & & & & 7082.23 & $8 h$ & 14115.95 & $30302_{5 / 2}-44418_{7 / 2}^{\circ}$ & & & & \\
\hline 7286.16 & 200 & 13720.87 & $15587_{11 / 2}-29308_{9 / 2}^{\circ}$ & so & 0.175 & 1.08 & & 7077.57 & $5 h$ & 14125.25 & & & & & \\
\hline 7284.30 & 100 & 13724.37 & $19132_{3 / 2}-32856_{5 / 2}^{\circ}$ & SI & 0.86 & 1.06 & * & 7072.74 & 1 & 14134.90 & $26368^{\circ}{ }_{11 / 2}-40503_{11 / 2}$ & & & & \\
\hline 7283.78 & $60 h$ & 13725.35 & $25745_{5 / 2}-39470^{\circ}{ }_{5 / 2}^{\circ / 2}$ & & & & & 7066.52 & $2 h$ & 14147.34 & $25699_{9 / 2}^{\circ}-39847_{7 / 2}$ & & & & \\
\hline 7282.85 & $10 h$ & 13727.11 & $25717_{\tau_{/ 2}}-39444_{9 / 2}^{\circ}$ & & & & & 7065.04 & $4 h$ & 14150.30 & $24348_{9 / 2}-38499_{9 / 2}^{\circ}$ & & & & \\
\hline 7281.82 & 20 & 13729.05 & $23335^{\circ}{ }_{7 / 2}-37064_{5 / 2}$ & so & 1.30 & 0.07 & & 7061.27 & $3 h$ & 14157.85 & & & & & \\
\hline 7280.34 & 3 & 13731.84 & & & & & & 7060.97 & 100 & 14158.46 & $8771_{s / 2}^{\circ}-22929_{5 / 2}$ & $\Delta \mathrm{J}=0$ & 0.85 & 1.142 & * \\
\hline 7279.56 & $4 h$ & 13733.31 & $26368^{\circ}{ }_{11 / 2}-40101_{9 / 2}$ & & & & & 7059.94 & 15 & 14160.52 & $19132_{3 / 2}-33292^{\circ}{ }_{5 / 2}$ & & & & \\
\hline 7279.02 & 3 & 13734.33 & & & & & & 7055.29 & 7 & 14169.85 & & & & & \\
\hline 7277.20 & 3 & 13737.76 & $23781_{9 / 2}-37519^{\circ}{ }^{\circ}$ & & & & & 7054.30 & 7 & 14171.84 & & & & & \\
\hline 7277.00 & 2 & 13738.14 & & & & & & 7052.05 & 4 & 14176.37 & $27440_{9 / 2}^{\circ}-41617_{7 / 2}$ & & & & \\
\hline 7276.15 & 7 & 13739.75 & & & & & & 7051.38 & 1 & 14177.71 & $25520_{11 / 2}^{\circ}-39698_{9 / 2}$ & & & & \\
\hline 7273.92 & 30 & 13743.96 & $19548_{5 / 2}-33292_{5 / 2}^{\circ}$ & $\Delta \mathrm{J}=0$ & 0.99 & 0.624 & * & 7050.54 & $30 h$ & 14179.40 & $28555_{7 / 2}-42735_{5 / 2}^{\circ}$ & & & & \\
\hline 7272.62 & 500 & 13746.42 & $17752_{5 / 2}-31499^{\circ}{ }_{3 / 2}^{\circ}$ & so & 0.97 & 0.22 & & 7039.74 & 3 & 14201.15 & $26448^{\circ}{ }_{5 / 2}-40649_{7 / 2}$ & & & & \\
\hline 7269.00 & $4 h$ & 13753.26 & $25717_{\tau_{/ 2}}-39470_{5 / 2}^{\circ}$ & $\mathrm{T}$ & 0.98 & & & 7034.34 & 150 & 14212.06 & & $\Delta \mathrm{J}=0$ & 1.11 & 0.10 & \\
\hline 7265.46 & 2 & 13759.96 & $26368^{\circ}{ }_{11 / 2}-40128_{11 / 2}^{/ 2}$ & & & & & 7034.12 & 8 & 14212.50 & $19748_{9 / 2}-33961_{7 / 2}^{\circ}$ & & & & \\
\hline 7263.81 & $1 h$ & 13763.09 & $25717_{7 / 2}-39480^{\circ}{ }_{7 / 2}$ & & & & & 7031.41 & 1 & 14217.98 & $26368_{11 / 2}^{\circ}-40586_{13 / 2}$ & & & & \\
\hline 7260.20 & 6 & 13769.93 & & & & & & 7029.40 & $80 h$ & 14222.04 & & $\Delta \mathrm{J}=0$ & 1.33 & 0.17 & \\
\hline 7259.63 & 20 & 13771.01 & $25717_{\tau / 2}-39488^{\circ}{ }_{9 / 2}$ & & & & & 7027.38 & 1 & 14226.13 & $21997_{11 / 2}-36223^{\circ}{ }_{13 / 2}$ & & & & \\
\hline 7257.72 & 50 & 13774.64 & $17613_{9 / 2}-31388_{9 / 2}^{\circ}$ & $\Delta \mathrm{J}=0$ & $?$ & 0.85 & & 7026.14 & 4 & 14228.64 & $26357^{\circ}{ }_{13 / 2}-40586_{13 / 2}$ & & & & \\
\hline 7249.43 & 3 & 13790.39 & & & & & & 7022.44 & 10 & 14236.14 & & & & & \\
\hline 7249.03 & 10 & 13791.15 & & & & & & 7021.74 & 3 & 14237.56 & & & & & \\
\hline 7245.97 & $40 h$ & 13796.97 & $25745_{s / 2}-39542^{\circ}{ }{ }^{2}$ & $\mathrm{~T}$ & 1.065 & & & 7019.73 & $10 h$ & 14241.63 & & & & & \\
\hline 7243.47 & 2 & 13801.73 & $30972_{11 / 2}-44774_{9 / 2}^{\circ}$ & & & & & 7017.90 & 150 & 14245.35 & $26448_{5 / 2}^{\circ}-40693_{3 / 2}$ & So & 1.14 & 1.32 & * \\
\hline 7237.44 & $2 h$ & 13813.23 & & & & & & 7012.18 & 50 & 14256.97 & $18693_{15 / 2}-32950^{\circ}{ }_{13 / 2}$ & & & & \\
\hline 7231.33 & $50 h$ & 13824.91 & $25717_{\tau_{/ 2}}-39542^{\circ}{ }_{\tau / 2}$ & $\Delta \mathrm{J}=0$ & 1.03 & 0.15 & & 7011.00 & 4 & 14259.37 & $27440_{9 / 2}^{\circ / 2}-41700_{11 / 2}$ & & & & \\
\hline 7230.36 & $5 h$ & 13826.76 & & & & & & 7010.79 & 70 & 14259.80 & $31454_{9 / 2}^{\circ}-45714_{11 / 2}$ & SI & 1.36 & 0.10 & \\
\hline 7214.17 & $100 h$ & 13857.79 & $25745_{5 / 2}-39602^{\circ}{ }_{3 / 2}$ & so & 0.79 & 0.30 & & 6997.38 & 40 & 14287.12 & $27440_{9 / 2}^{\circ}-41728_{9 / 2}$ & $\Delta \mathrm{J}=0$ & 1.08 & 0.18 & \\
\hline 7212.63 & $4 h$ & 13860.75 & $25699^{\circ}{ }_{9 / 2}-39560_{\tau^{\prime} / 2}$ & & & & & 6994.59 & $30 h$ & 14292.82 & & & & & \\
\hline 7211.50 & $1 h$ & 13862.92 & $24348_{9 / 2}-38211^{\circ}{ }_{7 / 2}$ & & & & & 6992.80 & 8 & 14296.48 & $25699_{9 / 2}^{\circ}-39996_{\tau^{\prime / 2}}$ & & & & \\
\hline 7211.15 & $2 h$ & 13863.59 & & & & & & 6989.13 & 40 & 14303.99 & & $\mathrm{~T}$ & 1.15 & & \\
\hline 7209.58 & 6 & 13866.61 & & & & & & 6980.04 & $3 h$ & 14322.62 & & & & & \\
\hline 7208.74 & 9 & 13868.23 & & & & & & 6975.33 & $3 h$ & 14332.29 & $21997_{11 / 2}-36329^{\circ}{ }_{11 / 2}$ & & & & \\
\hline 7205.35 & 7 & 13874.75 & $26889_{9 / 2}-40763^{\circ}{ }_{7 / 2}$ & & & & & 6971.78 & $10 h$ & 14339.58 & $25207^{\circ}{ }_{3 / 2}-39547_{\mathrm{s} / 2}$ & so & 1.20 & 0.09 & \\
\hline 7202.19 & 30 & 13880.84 & & & & & & 6969.74 & 7 & 14343.78 & & & & & \\
\hline 7201.12 & $20 h$ & 13882.90 & $25745_{5 / 2}-39628^{\circ}{ }_{7 / 2}$ & & & & & 6954.13 & 4 & 14375.98 & $23335_{7 / 2}^{\circ}-37711_{9 / 2}$ & & & & \\
\hline 7199.20 & 4 & 13886.61 & $24957^{\circ}{ }_{11 / 2}-38844_{11 / 2}$ & & & & & 6949.54 & 30 & 14385.47 & & & & & \\
\hline 7198.47 & 30 & 13888.01 & & $\Delta \mathrm{J}=\mathrm{C}$ & $?$ & 0.31 & & 6946.15 & 2 & 14392.49 & $24137_{13 / 2}-38529^{\circ}{ }_{11 / 2}$ & & & & \\
\hline 7186.89 & 40 & 13910.39 & & $\Delta \mathrm{J}=0$ & $?$ & 0.22 & & 6941.79 & $3 h$ & 14401.53 & $23941_{9 / 2}^{\circ}-38342_{\tau_{/ 2}}$ & & & & \\
\hline 7186.09 & $2 h$ & 13911.94 & $27440_{9 / 2}^{\circ}-41352_{11 / 2}$ & & & & & 6941.55 & 2 & 14402.03 & $25699_{9 / 2}^{\circ}-40101_{9 / 2}$ & & & & \\
\hline 7185.17 & $20 h$ & 13913.72 & $25745_{5 / 2}-39658^{\circ / 2}$ & $\Delta \mathrm{J}=0$ & $?$ & 0.34 & & 6937.37 & 80 & 14410.71 & $16957_{\tau / 2}^{2 / 2}-31367^{\circ}{ }_{7 / 2}^{\circ}$ & $\Delta \mathrm{J}=0$ & 1.25 & 0.57 & \\
\hline 7174.78 & $3 h$ & 13933.87 & $21120_{7_{/ 2}}-35054_{9 / 2}^{\circ}$ & & & & & 6933.32 & 9 & 14419.13 & & $\mathrm{~T}$ & 1.15 & & \\
\hline 7172.48 & 2 & 13938.34 & & & & & & 6932.13 & 4 & 14421.60 & $25520^{\circ}{ }_{11 / 2}-39942_{11 / 2}$ & & & & \\
\hline 7169.27 & 20 & 13944.58 & $21737_{9 / 2}-35682^{\circ}{ }_{7 / 2}$ & $\mathrm{~T}$ & 1.10 & & & 6928.66 & 30 & 14428.83 & $17752_{5 / 2}-32181_{3 / 2}^{\circ}$ & SI & 1.186 & 0.723 & * \\
\hline 7161.52 & $2 h$ & 13959.67 & $18990_{11 / 2}-32950^{\circ}{ }_{13 / 2}^{2}$ & & & & & 6927.90 & 6 & 14430.41 & $17343_{7 / 2}-31773_{5 / 2}^{\circ}$ & & & & \\
\hline 7160.63 & 1 & 13961.40 & $21997_{11 / 2}-35958^{\circ}{ }_{13 / 2}^{\circ}$ & & & & & 6927.46 & 3 & 14431.32 & $16957_{7 / 2}-31388_{9 / 2}^{\circ / 2}$ & & & & \\
\hline 7159.01 & 5 & 13964.56 & $16957_{\tau / 2}-30921^{\circ}{ }_{7 / 2}$ & & & & & 6923.16 & 10 & 14440.29 & $19753_{\tau / 2}-34194_{5 / 2}^{\circ}$ & & & & \\
\hline 7152.04 & 20 & 13978.17 & $19748_{9 / 2}-33726_{9 / 2}^{\circ}$ & $\Delta \mathrm{J}=0$ & $?$ & 0.68 & & & & & $30125_{13 / 2}-44565_{15 / 2}^{\circ}$ & & & & \\
\hline 7147.55 & 2 & 13986.95 & $23781_{9 / 2}-37768_{9 / 2}^{\circ}$ & & & & & 6915.86 & 40 & 14455.53 & $27037^{\circ}{ }_{7 / 2}-41493_{5 / 2}$ & So & 0.67 & 0.43 & \\
\hline 7146.32 & $3 h$ & 13989.36 & $27440_{9 / 2}^{\circ}-41430_{11 / 2}^{\circ}$ & & & & & 6914.35 & $2 h$ & 14458.69 & $23309_{11 / 2}-37768_{9 / 2}$ & & & & \\
\hline 7145.92 & 10 & 13990.14 & $16957_{\tau_{/ 2}}-30947_{9 / 2}^{\circ}$ & & & & & 6898.56 & 80 & 14491.78 & & $\Delta \mathrm{J}=0$ & 1.07 & 0.33 & \\
\hline 7139.95 & 60 & 14001.84 & & $\Delta \mathrm{J}=0$ & 1.18 & 0.10 & & 6897.70 & 20 & 14493.59 & $17613_{9 / 2}-32107^{\circ}{ }_{11 / 2}$ & & & & \\
\hline 7138.58 & $2 h$ & 14004.53 & $22929_{5 / 2}-36934^{\circ}{ }_{\tau / 2}$ & & & & & 6895.95 & 4 & 14497.27 & $16957_{\tau / 2}-31454_{9 / 2}^{\circ}$ & & & & \\
\hline 7138.21 & $5 h$ & 14005.25 & & & & & & 6894.18 & $5 h$ & 14500.99 & $26488_{3_{3 / 2}}-40989_{3 / 2}^{\circ}$ & & & & \\
\hline 7134.25 & $3 h$ & 14013.03 & & & & & & 6891.27 & 7 & 14507.11 & $27037^{\circ / 2}-41544_{s / 2}$ & & & & \\
\hline 7132.62 & $2 h$ & 14016.23 & & & & & & 6888.71 & 9 & 14512.50 & & & & & \\
\hline 7130.73 & 50 & 14019.94 & $8771_{5 / 2}^{\circ}-22791_{7 / 2}$ & SI & 0.85 & 1.13 & * & 6884.75 & 10 & 14520.85 & $21161_{5 / 2}-35682^{\circ}{ }_{7 / 2}$ & & & & \\
\hline 7130.13 & $1 h$ & 14021.12 & $17752_{5 / 2}-31773^{\circ}{ }_{5 / 2}$ & & & & & 6881.11 & 5 & 14528.53 & $24348_{9 / 2}-38877^{\circ}{ }_{11 / 2}$ & & & & \\
\hline 7128.46 & $100 h$ & 14024.41 & $17343_{7 / 2}-31367_{7 / 2}^{\circ}$ & $\Delta \mathrm{J}=0$ & $?$ & 1.09 & & 6871.47 & 2 & 14548.91 & & & & & \\
\hline 7128.32 & $40 h$ & 14024.69 & $25717_{\tau / 2}^{1 / 2}-39741_{9 / 2}^{\circ / 2}$ & SI & 1.44 & 0.33 & & 6869.69 & $3 h$ & 14552.68 & & & & & \\
\hline 7123.95 & 2 & 14033.29 & & & & & & 6865.58 & 10 & 14561.39 & $21120_{\gamma_{2}}-35682^{\circ}{ }_{\gamma / 2}$ & & & & \\
\hline 7122.09 & 20 & 14036.95 & $27037^{\circ}{ }_{7 / 2}-41074_{9 / 2}$ & & & & & 6861.95 & $2 h$ & 14569.10 & & & & & \\
\hline 7115.41 & 15 & 14050.13 & & & & & & 6856.863 & 50 & 14579.91 & $19466_{13 / 2}-34046^{\circ}{ }_{15 / 2}$ & $\mathrm{~T}$ & 1.22 & & \\
\hline 7109.63 & $2 h$ & 14061.55 & $28514_{9 / 2}^{\circ}-42576_{9 / 2}$ & & & & & 6854.115 & 200 & 14585.75 & $26488_{3 / 2}-41074_{5 / 2}^{\circ}$ & SI & 1.20 & 0.21 & \\
\hline 7108.06 & $2 n$ & 14064.66 & $17454_{13 / 2}-31519^{\circ}{ }_{11 / 2}$ & & & & & 6853.56 & 3 & 14586.93 & & & & & \\
\hline 7106.14 & 150 & 14068.46 & $18693_{15 / 2}-32761^{\circ}{ }_{17 / 2}$ & So & 0.56 & 0.52 & & 6852.89 & 2 & 14588.36 & & & & & \\
\hline
\end{tabular}


APPENDIX - Spectral Lines of Neutral Thulium - Continued

\begin{tabular}{|c|c|c|c|c|c|c|c|c|c|c|c|c|c|c|}
\hline \multirow{2}{*}{$\begin{array}{c}\text { Wave- } \\
\text { length } \\
\text { in air } \\
\AA\end{array}$} & \multirow[t]{2}{*}{ Intensity } & \multirow{2}{*}{$\begin{array}{l}\text { Wave- } \\
\text { number } \\
\mathrm{cm}^{-1}\end{array}$} & \multirow[t]{2}{*}{ Classification } & \multirow{2}{*}{\multicolumn{3}{|c|}{ Zeeman effect }} & \multirow{2}{*}{$\begin{array}{c}\text { Wave- } \\
\text { length } \\
\text { in air } \\
\AA\end{array}$} & Intensity & $\begin{array}{l}\text { Wave- } \\
\text { number }\end{array}$ & Classification & & eeman $\epsilon$ & ffect & \\
\hline & & & & & & & & & $\mathrm{cm}^{-1}$ & & type & f & $\mathrm{Je}$ & \\
\hline 6852.23 & 7 & 14589.76 & $22929_{5 / 2}-37519^{\circ}{ }_{\gamma_{/ 2}}$ & & & & 6610.112 & $10 h$ & 15124.16 & $24418_{5 / 2}-39542_{7 / 2}^{\circ}$ & $\mathrm{T}$ & 1.03 & & \\
\hline 6851.12 & 4 & 14592.13 & $21737_{9 / 2}-36329^{\circ}{ }_{11 / 2}$ & & & & 6607.045 & 3 & 15131.18 & $24348_{9 / 2}-39479^{\circ}{ }_{11 / 2}$ & & & & \\
\hline 6845.755 & 1000 & 14603.56 & $17613_{9 / 2}-32217_{9 / 2}^{\circ}$ & $\Delta \mathrm{J}=0$ & 1.19 & 1.33 & 6604.955 & 2000 & 15135.97 & $17343_{7 / 2}-32479_{5 / 2}^{\circ}$ & SI & 1.02 & 0.89 & * \\
\hline 6844.262 & 2000 & 14606.75 & $17752_{5 / 2}-32359^{\circ}{ }_{7 / 2}^{2}$ & $\mathrm{~T}$ & 1.23 & & 6603.397 & 3 & 15139.54 & $24348_{9 / 2}-39488_{9 / 2}^{\circ}$ & & & & \\
\hline 6835.494 & 100 & 14625.49 & $16742_{7 / 2}-31367_{7 / 2}^{\circ}$ & $\mathrm{T}$ & 1.335 & & 6602.211 & 1 & 15142.26 & & & & & \\
\hline 6831.092 & 300 & 14634.91 & $26439_{7 / 2}-41074_{5 / 2}^{\circ / 2}$ & $\mathrm{~T}$ & 1.01 & & 6600.516 & 5 & 15146.15 & & & & & \\
\hline 6826.954 & 200 & 14643.78 & $24418_{5 / 2}-39061_{3 / 2}^{\circ}$ & So & 1.06 & 1.34 & 6598.134 & 20 & 15151.62 & $22559^{\circ}{ }_{11 / 2}-37711_{9 / 2}$ & $\mathrm{~T}$ & 1.13 & & \\
\hline 6820.266 & 200 & 14658.14 & $17454_{13 / 2}-32112^{\circ}{ }_{15 / 2}$ & & & & 6591.239 & 4 & 15167.47 & & & & & \\
\hline 6805.234 & 20 & 14690.52 & $27037^{\circ}{ }_{7 / 2}-41728_{9 / 2}$ & & & & 6590.585 & 1 & 15168.97 & $26448_{s / 2}^{\circ}-41617_{7 / 2}$ & & & & \\
\hline 6794.576 & 20 & 14713.56 & $27037^{\circ}{ }_{7 / 2}-41751_{5 / 2}$ & $\mathrm{~T}$ & 1.16 & 0.5 & 6589.744 & 5 & 15170.91 & $24957^{\circ}{ }_{11 / 2}^{/ 2}-40128_{11 / 2}$ & & & & \\
\hline 6788.516 & 300 & 14726.70 & $17752_{5 / 2}-32479_{5 / 2}^{\circ}$ & $\Delta \mathrm{J}=0$ & 1.185 & 0.90 & 6583.684 & 20 & 15184.87 & $24418_{5 / 2}-39602_{3 / 2}^{\circ}$ & & & & \\
\hline 6783.436 & 10 & 14737.72 & $16957_{\tau / 2}^{2}-31694_{\tau^{2}}^{\circ}$ & $\mathrm{T}$ & 1.15 & & 6581.884 & 1 & 15189.02 & $23309_{11 / 2}-38499^{\circ}{ }_{9 / 2}$ & & & & \\
\hline 6781.998 & 300 & 14740.85 & $24348_{9 / 2}-39089^{\circ}{ }_{11 / 2}$ & $\mathrm{~T}$ & 1.10 & & 6580.963 & 5 & 15191.15 & $30972_{11 / 2}-46163^{\circ}{ }_{11 / 2}$ & & & & \\
\hline 6779.767 & 2000 & 14745.70 & $17613_{9 / 2}-32359^{\circ}{ }_{7 / 2}$ & $\mathrm{~T}$ & 1.19 & & 6580.057 & 1 & 15193.24 & & & & & \\
\hline 6777.927 & 100 & 14749.70 & $26439_{\eta^{2} / 2}-41189_{\eta_{/ 2}}^{\circ}$ & $\Delta \mathrm{J}=0$ & $?$ & 0.7 & 6579.961 & 2 & 15193.46 & $25745_{5 / 2}-40938_{5 / 2}^{\circ}$ & & & & \\
\hline 6767.477 & 100 & 14772.48 & $26439_{7 / 2}-41211^{\circ}{ }_{7 / 2}$ & $\Delta \mathrm{J}=0$ & 1.00 & 1.18 & & & & $24348_{9 / 2}-39542^{\circ}{ }_{7 / 2}^{\circ}$ & & & & \\
\hline 6761.466 & 10 & 14785.61 & $23335^{\circ}{ }_{7 / 2}^{\circ}-38120_{9 / 2}^{/ 2}$ & & & & 6578.644 & 7 & 15196.50 & $21737_{9 / 2}-36934^{\circ / 2}{ }_{7 / 2}$ & & & & \\
\hline 6754.274 & 10 & 14801.35 & $18837_{9 / 2}-33638_{11 / 2}^{\circ}$ & & & & 6577.161 & 1 & 15199.93 & & & & & \\
\hline 6744.991 & 4 & 14821.73 & $18990_{11 / 2}-33812^{\circ}{ }_{13 / 2}$ & & & & 6575.542 & 100 & 15203.67 & & so & 0.66 & 0.31 & \\
\hline 6742.114 & 30 & 14828.05 & $17613_{9 / 2}-32441_{9 / 2}^{\circ}$ & & & & 6573.521 & 2 & 15208.35 & & & & & \\
\hline 6740.853 & 3 & 14830.82 & & & & & 6572.828 & 4 & 15209.95 & $24418_{5 / 2}-39628^{\circ}{ }_{7 / 2}$ & & & & \\
\hline 6739.222 & 50 & 14834.41 & & SI & 1.13 & 0.27 & 6570.961 & 2 & 15214.27 & $24348_{9 / 2}-39563_{11 / 2}^{\circ}$ & & & & \\
\hline 6726.338 & 100 & 14862.83 & $26126_{5 / 2}-40989_{3 / 2}^{\circ}$ & SI & 1.09 & 0.41 & 6568.632 & 1 & 15219.67 & $23309_{11 / 2}-38529^{\circ}{ }_{11 / 2}^{11 / 2}$ & & & & \\
\hline 6723.371 & 3 & 14869.39 & $23882_{3 / 2}-38751_{s / 2}^{\circ}$ & & & & 6566.287 & 100 & 15225.10 & $19466_{13 / 2}-34691^{\circ}{ }_{11 / 2}$ & $\mathrm{~T}$ & 1.17 & & \\
\hline 6721.365 & 1000 & 14873.82 & $17343_{7 / 2}-32217_{9 / 2}^{\circ}$ & SI & 1.022 & $1.333 *$ & 6565.525 & 10 & 15226.87 & $27440_{9 / 2}^{\circ}-42667_{9 / 2}$ & & & & \\
\hline 6692.931 & 200 & 14937.01 & $19132_{3 / 2}-34069^{\circ}{ }_{1 / 2}^{\circ}$ & SI & 1.02 & 0.125 & 6557.386 & 2 & 15245.77 & $25130_{15 / 2}-40376_{13 / 2}^{\circ}$ & & & & \\
\hline 6688.241 & 10 & 14947.49 & $26126_{s / 2}-41074_{s / 2}^{\circ}$ & & & & 6555.488 & 20 & 15250.18 & $18693_{15 / 2}-33943^{\circ}{ }_{13 / 2}$ & & & & \\
\hline 6685.990 & 4 & 14952.52 & $16742_{7 / 2}-31694^{\circ}{ }_{7 / 2}$ & & & & 6555.395 & 2 & 15250.40 & & & & & \\
\hline 6684.533 & 30 & 14955.78 & $18837_{9 / 2}-33793_{11 / 2}^{\circ}$ & & & & 6552.617 & 2 & 15256.86 & $21120_{7_{7 / 2}}-36377_{9 / 2}^{\circ}$ & & & & \\
\hline 6678.652 & 10 & 14968.95 & $24418_{5 / 2}-39386_{s / 2}^{\circ}$ & & & & 6551.623 & 5 & 15259.18 & & & & & \\
\hline 6678.431 & 3 & 14969.44 & & & & & 6551.185 & 100 & 15260.20 & $16957_{\tau_{/ 2}}-32217_{9 / 2}^{\circ}$ & & & & \\
\hline 6672.681 & 30 & 14982.34 & $25520^{\circ}{ }_{11 / 2}-40503_{11 / 2}$ & $\Delta \mathrm{J}=0$ & 1.10 & 0.05 & 6547.015 & 1 & 15269.92 & & & & & \\
\hline 6672.337 & 50 & 14983.12 & $19466_{13 / 2}-34449^{\circ}{ }_{13 / 2}^{1 / 2}$ & $\Delta \mathrm{J}=0$ & 1.16 & 0.14 & 6543.725 & $5 h$ & 15277.60 & $27440_{9 / 2}^{\circ}-42718_{9 / 2}$ & & & & \\
\hline 6671.768 & 3 & 14984.39 & $26368^{\circ}{ }_{11 / 2}-41352_{11 / 2}$ & & & & 6541.883 & 3 & 15281.90 & $22929_{5 / 2}-38211^{\circ}{ }_{7 / 2}$ & & & & \\
\hline 6659.025 & 7 & 15013.07 & $21997_{11 / 2}-37010_{9 / 2}^{\circ}$ & & & & 6539.825 & 40 & 15286.71 & & & & & \\
\hline 6658.639 & 500 & 15013.94 & $24348_{9 / 2}-39362_{11 / 2}^{\circ}$ & $\mathrm{T}$ & 1.12 & & 6539.134 & 1 & 15288.32 & $24708^{\circ}{ }_{7 / 2}-39996_{7 / 2}$ & & & & \\
\hline 6657.720 & 2000 & 15016.01 & $17343_{7 / 2}-32359^{\circ}{ }_{7 / 2}$ & $\Delta \mathrm{J}=0$ & 1.02 & 1.19 & 6535.449 & 20 & 15296.94 & $24137_{13 / 2}-39434^{\circ}{ }_{13 / 2}$ & & & & \\
\hline 6657.095 & 40 & 15017.42 & $22559^{\circ}{ }_{11 / 2}^{\circ}-37576_{9 / 2}$ & & & & 6534.406 & 100 & 15299.38 & & $\mathrm{~T}$ & 1.17 & 0.07 & \\
\hline 6656.502 & 2 & 15018.76 & $25745_{s_{/ 2}}-40763^{\circ}{ }_{\tau / 2}^{\circ}$ & & & & 6534.215 & 10 & 15299.83 & & & & & \\
\hline 6654.745 & 1 & 15022.72 & $26368^{\circ}{ }_{11 / 2}-41391_{9 / 2}$ & & & & 6533.784 & 1 & 15300.84 & $19753_{\tau_{/ 2}}-35054_{9 / 2}^{\circ}$ & & & & \\
\hline 6653.692 & 10 & 15025.10 & & & & & 6531.528 & 2 & 15306.12 & $19748_{9 / 2}-35054_{9 / 2}^{\circ}$ & & & & \\
\hline 6652.675 & 30 & 15027.40 & $17752_{5 / 2}-32780^{\circ}{ }_{3 / 2}$ & & & & 6530.807 & 50 & 15307.81 & $23781_{9 / 2}-39089^{\circ}{ }_{11 / 2}$ & so & 0.82 & 0.25 & \\
\hline 6650.852 & 100 & 15031.52 & $16742_{7 / 2}-31773_{5 / 2}^{\circ / 2}$ & & & & 6525.863 & 2 & 15319.41 & $25536_{15 / 2}^{\circ}-40855_{13 / 2}$ & & & & \\
\hline 6645.212 & 40 & 15044.27 & & & & & 6525.648 & 5 & 15319.92 & $18990_{11 / 2}-34310_{9 / 2}^{\circ}$ & & & & \\
\hline 6644.996 & 30 & 15044.76 & $26448_{5 / 2}^{\circ}-41493_{5 / 2}$ & & & & 6523.994 & $20 h$ & 15323.80 & $27440^{\circ}{ }_{9 / 2}-42764_{9 / 2}$ & & & & \\
\hline 6643026 & & 15 & $24418_{5 / 2}-39467^{\circ}{ }_{7 / 2}$ & & & & $\begin{array}{l}6519.778 \\
6519.421\end{array}$ & $\begin{array}{r}200 \\
1\end{array}$ & $\begin{array}{l}15333.71 \\
15334.55\end{array}$ & $\begin{array}{l}25656_{5 / 2}-40989^{\circ}{ }_{3 / 2} \\
25520^{\circ}-40855^{\circ}\end{array}$ & SI & 0.72 & 0.40 & * \\
\hline $\begin{array}{l}6643.026 \\
6642.556\end{array}$ & $\begin{array}{l}5 \\
7\end{array}$ & $\begin{array}{l}15049.23 \\
15050.29\end{array}$ & $25536^{\circ}{ }_{15 / 2}-40586_{13 / 2}$ & & & & $\begin{array}{l}0519.421 \\
6517.443\end{array}$ & $\begin{array}{l}1 \\
5\end{array}$ & $\begin{array}{l}15334.55 \\
15339.20\end{array}$ & $\begin{array}{l}25520_{11 / 2}-40855_{13 / 2} \\
21799_{3 / 2}^{\circ}-37138_{3 / 2}\end{array}$ & & & & \\
\hline 6641.592 & 30 & 15052.47 & $24418_{5 / 2}-39470_{5 / 2}^{\circ}$ & $\mathrm{T}$ & 1.08 & & 6515.094 & 3 & 15344.73 & & & & & \\
\hline 6637.463 & 3 & 15061.84 & $26368^{\circ}{ }_{11 / 2}-41430_{11 / 2}^{/ 2}$ & & & & 6514.208 & 2 & 15346.82 & $23873_{9 / 2}-39220_{9 / 2}^{\circ}$ & & & & \\
\hline 6637.273 & 100 & 15062.27 & $26126_{5 / 2}-41189^{\circ}{ }_{7 / 2}$ & SI & 1.39 & 0.20 & 6513.678 & 1 & 15348.07 & & & & & \\
\hline 6636.080 & 3 & 15064.98 & $24348_{9 / 2}-39413^{\circ}{ }_{7 / 2}$ & & & & 6513.640 & 1 & 15348.16 & & & & & \\
\hline 6635.528 & 30 & 15066.23 & $19466_{13 / 2}-34532^{\circ}{ }_{11 / 2}$ & $\mathrm{~T}$ & 1.25 & & 6511.384 & 40 & 15353.48 & $18693_{15 / 2}-34046^{\circ}{ }_{15 / 2}$ & $\mathrm{~T}$ & 1.16 & & \\
\hline 6632.770 & 2 & 15072.50 & $26357^{\circ}{ }_{13 / 2}^{\circ}-41430_{11 / 2}$ & & & & 6507.663 & 300 & 15362.26 & & So & 0.73 & 0.11 & \\
\hline 6630.445 & 2 & 15077.78 & & & & & 6507.302 & 20 & 15363.11 & & & & & \\
\hline 6627.248 & 100 & 15085.05 & $26126_{5 / 2}-41211^{\circ}{ }_{7 / 2}$ & SI & 1.40 & 0.22 & 6503.803 & 2 & 15371.37 & $23873_{7 / 2}-39244_{9 / 2}^{\circ}$ & & & & \\
\hline 6625.313 & 5 & 15089.46 & $18853_{11 / 2}-33943^{\circ}{ }_{13 / 2}$ & & & & 6498.085 & 1 & 15384.90 & $24611^{\circ}{ }^{\circ / 2}-39996_{7 / 2}$ & & & & \\
\hline 6624.609 & 1 & 15091.06 & & & & & 6496.873 & 3 & 15387.77 & $26448^{\circ}{ }_{s / 2}-41836_{s / 2}$ & & & & \\
\hline 6622.619 & 20 & 15095.60 & $24348_{9 / 2}-39444_{9 / 2}^{\circ}$ & & & & 6494.573 & 40 & 15393.22 & $24348_{9 / 2}-39741_{9 / 2}^{\circ}$ & $\mathrm{T}$ & 1.10 & & \\
\hline 6621.420 & 30 & 15098.33 & $17343_{\tau / 2}-32441_{9 / 2}^{\circ / 2}$ & $\Delta \mathrm{J}=1$ & $?$ & 0.80 & 6493.905 & 3 & 15394.80 & $13119_{9 / 2}-28514_{9 / 2}^{\circ}$ & & & & \\
\hline 6620.230 & 2 & 15101.05 & $26368^{\circ}{ }_{11 / 2}-41469_{13 / 2}$ & & & & 6490.703 & 200 & 15402.40 & $16957_{7 / 2}-32359^{\circ}{ }_{7 / 2}$ & $\Delta \mathrm{J}=0$ & 1.18 & 0.10 & \\
\hline 6619.814 & 10 & 15101.99 & $8771_{5 / 2}^{\circ}-23873_{7 / 2}$ & & & & 6489.080 & $20 h$ & 15406.25 & & & & & \\
\hline 6619.698 & 5 & 15102.26 & $27440_{9 / 2}^{\circ / 2}-42543_{7 / 2}$ & & & & 6485.678 & 1 & 15414.33 & & & & & \\
\hline 6619.433 & 1 & 15102.86 & $25699_{9 / 2}^{\circ}-40802_{7 / 2}$ & & & & 6483.978 & 40 & 15418.37 & $25656_{5 / 2}-41074_{5 / 2}^{\circ}$ & & & & \\
\hline 6618.946 & 2 & 15103.97 & $17752_{5 / 2}-32856^{\circ}{ }_{5 / 2}^{2}$ & & & & 6480.185 & 40 & 15427.40 & $27440_{9 / 2}^{\circ}-42868_{11 / 2}$ & & & & \\
\hline 6616.797 & 40 & 15108.88 & $22468^{\circ}{ }_{11 / 2}-37576_{9 / 2}$ & & & & 6478.173 & 1 & 15432.19 & & & & & \\
\hline 6615.563 & 10 & 15111.70 & $26357_{13 / 2}^{\circ}-41469_{13 / 2}$ & & & & 6476.494 & 20 & 15436.19 & & & & & \\
\hline 6613.516 & 2 & 15116.38 & & & & & 6473.192 & 3 & 15444.06 & $25745_{5 / 2}-41189^{\circ}{ }_{7 / 2}$ & & & & \\
\hline 6612.340 & 4 & 15119.06 & $18693_{15 / 2}-33812^{\circ}{ }_{13 / 2}$ & & & & 6472.815 & 1 & 15444.96 & $25699^{\circ}{ }_{9 / 2}-41144_{7 / 2}$ & & & & \\
\hline 6610.318 & 40 & 15123.69 & $18837_{9 / 2}-33961_{7 / 2}^{\circ}$ & $\mathrm{T}$ & 1.12 & & 6470.630 & 5 & 15450.18 & $19548_{5 / 2}-34999^{\circ}{ }_{3 / 2}^{\circ}$ & & & & \\
\hline
\end{tabular}


APPENDIX - Spectral Lines of Neutral Thulium - Continued

\begin{tabular}{|c|c|c|c|c|c|c|c|c|c|c|c|c|c|c|}
\hline \multirow{2}{*}{$\begin{array}{c}\text { Wave- } \\
\text { length } \\
\text { in air } \\
\AA\end{array}$} & \multirow[t]{2}{*}{ Intensity } & \multirow{2}{*}{$\begin{array}{l}\text { Wave- } \\
\text { number } \\
\mathrm{cm}^{-1}\end{array}$} & \multirow[t]{2}{*}{ Classification } & \multirow{2}{*}{\multicolumn{4}{|c|}{ Zeeman effect }} & \multirow{2}{*}{$\begin{array}{c}\text { Wave- } \\
\text { length } \\
\text { in air } \\
\AA\end{array}$} & Intensity & $\begin{array}{l}\text { W ave- } \\
\text { number }\end{array}$ & Classification & & eeman ef & ffect \\
\hline & & & & & & & & & & $\mathrm{cm}^{-1}$ & & type & f & $\mathrm{Je}$ \\
\hline 6470.203 & 3 & 15451.20 & & & & & & 6355.308 & 1 & 15730.53 & $25699_{9 / 2}^{\circ}-41430_{11 / 2}$ & & & \\
\hline 6467.980 & 7 & 15456.51 & $18853_{11 / 2}-34310_{9 / 2}^{\circ}$ & & & & & 6353.842 & 6 & 15734.16 & $18853_{11 / 2}-34587_{9 / 2}^{\circ}$ & & & \\
\hline 6467.389 & 1 & 15457.92 & & & & & & 6353.722 & 1 & 15734.46 & $22929_{s / 2}-38664^{\circ}{ }_{7 / 2}$ & & & \\
\hline 6466.788 & 20 & 15459.36 & $18990_{11 / 2}-34449^{\circ}{ }_{13 / 2}$ & & & & & 6353.470 & 3 & 15735.08 & & & & \\
\hline 6465.814 & 10 & 15461.68 & $27440_{9 / 2}^{\circ}-42902_{9 / 2}$ & & & & & 6352.660 & 300 & 15737.09 & $16742_{7 / 2}-32479^{\circ}{ }_{5 / 2}$ & SI & 1.324 & 0.89 \\
\hline 6463.405 & 30 & 15467.45 & $18990_{11 / 2}-34457_{9 / 2}^{\circ}$ & SI & 1.30 & 0.09 & & 6349.066 & 30 & 15745.99 & $27037^{\circ}{ }_{7 / 2}^{2}-42783_{7 / 2}$ & & & \\
\hline 6462.940 & $1 h$ & 15468.56 & & & & & & 6347.203 & 1 & 15750.62 & $18837_{9 / 2}-34587_{9 / 2}^{\circ}$ & & & \\
\hline 6461.146 & 1 & 15472.85 & $26646_{9 / 2}-42119^{\circ}{ }_{11 / 2}$ & & & & & 6345.885 & 1 & 15753.89 & $29316_{9 / 2}-45070_{9 / 2}^{\circ / 2}$ & & & \\
\hline 6460.261 & 5000 & 15474.97 & $16742_{7 / 2}-32217_{9 / 2}^{\circ}$ & $\mathrm{T}$ & 1.35 & & & 6345.528 & 20 & 15754.77 & $23873_{\gamma^{2} / 2}-39628^{\circ}{ }_{7 / 2}$ & & & \\
\hline 6458.850 & $2 h$ & 15478.36 & & & & & & 6344.748 & 100 & 15756.71 & $18693_{15 / 2}-34449^{\circ}{ }_{13 / 2}$ & $\mathrm{~T}$ & 1.17 & \\
\hline 6458.032 & 1 & 15480.32 & $24611_{s / 2}^{\circ}-40091_{3 / 2}$ & & & & & 6344.373 & 1 & 15757.64 & $23941_{9 / 2}^{\circ}-39698_{9 / 2}$ & & & \\
\hline 64.56 .201 & 30 & 15484.71 & $16957_{\tau / 2}-32441_{9 / 2}^{\circ}$ & SI & 1.53 & 0.27 & & 6338.916 & 2 & 15771.21 & $21997_{11 / 2}-37768_{9 / 2}^{\circ}$ & & & \\
\hline 6455.985 & 2 & 15485.22 & & & & & & 6338.520 & 10 & 15772.19 & & $\mathrm{~T}$ & 1.14 & 0.03 \\
\hline 6454.936 & 20 & 15487.74 & $17752_{5 / 2}-33240^{\circ}{ }_{7 / 2}$ & $\mathrm{~T}$ & 1.14 & & & 6338.270 & 3 & 15772.81 & $21161_{5 / 2}-36934^{\circ}{ }_{7 / 2}$ & & & \\
\hline 6452.737 & 30 & 15493.02 & $15587_{11 / 2}-31080^{\circ}{ }_{11 / 2}^{\circ}$ & & & & & 6334.658 & 1 & 15781.81 & $21737_{9 / 2}-37519^{\circ}{ }_{7 / 2}^{\circ}$ & & & \\
\hline 6451.587 & 1 & 15495.78 & & & & & & 6334.454 & 1 & 15782.32 & $17613_{9 / 2}-33396_{\tau / 2}^{\circ}$ & & & \\
\hline 6444.334 & 30 & 15513.22 & $17343_{7 / 2}-32856_{5 / 2}^{\circ}$ & $\mathrm{T}$ & 0.98 & & & 6332.154 & 1 & 15788.05 & $23335^{\circ}{ }_{7 / 2}-39123_{9 / 2}$ & & & \\
\hline 6444.117 & 20 & 15513.74 & $23873_{7 / 2}-39386_{5 / 2}^{\circ}$ & & & & & 6330.596 & 1 & 15791.93 & & & & \\
\hline 6443.060 & 2 & 15516.29 & $27440_{9 / 2}^{\circ}-42957_{11 / 2}^{\circ}$ & & & & & 6325.911 & 1 & 15803.63 & & & & \\
\hline 6442.656 & 2 & 15517.26 & & & & & & 6323.217 & 1 & 15810.36 & & & & \\
\hline 6440.829 & 1 & 15521.66 & & & & & & 6322.015 & 1 & 15813.37 & $21120_{7 / 2}-36934_{7 / 2}^{\circ}$ & & & \\
\hline 6440.540 & 100 & 15522.36 & $16957_{\tau / 2}-32479^{\circ}{ }_{s / 2}$ & SI & 1.17 & 0.90 & * & 6318.529 & 3 & 15822.09 & $22929_{5 / 2}-38751_{s / 2}^{\circ / 2}$ & $\Delta \mathrm{J}=0$ & 1.18 & 0.215 \\
\hline 64.38 .105 & 200 & 15528.23 & $18837_{9 / 2}-34365_{7 / 2}^{\circ}$ & So & 0.88 & 0.25 & & 6314.649 & 7 & 15831.82 & $25520^{\circ}{ }_{11 / 2}-41352_{11 / 2}$ & $\Delta \mathrm{J}=0$ & 1.09 & 0.19 \\
\hline 6437.887 & 2 & 15528.76 & & & & & & 6312.195 & 20 & 15837.97 & $18853_{11 / 2}-34691^{\circ}{ }_{11 / 2}$ & $\mathrm{~T}$ & 1.153 & \\
\hline 6436.070 & $30 h$ & 15533.14 & $25656_{5 / 2}-41189^{\circ}{ }_{7 / 2}$ & & & & & 6310.012 & 1 & 15843.45 & & & & \\
\hline 6433.173 & 5 & 15540.13 & $17752_{s / 2}-33292_{s / 2}^{\circ}$ & & & & & 6306.266 & 1 & 15852.86 & $26889_{9 / 2}-42742^{\circ}{ }_{7 / 2}$ & & & \\
\hline 6432.197 & 1 & 15542.49 & $18990_{11 / 2}-34532^{\circ}{ }_{11 / 2}$ & & & & & 6305.650 & 50 & 15854.41 & $18837_{9 / 2}-34691_{11 / 2}^{\circ}$ & SI & 1.205 & 0.045 \\
\hline 6427.655 & 4 & 15553.47 & $25520^{\circ}{ }_{11 / 2}-41074_{9 / 2}$ & & & & & 6302.513 & 10 & 15862.30 & $27440_{9 / 2}^{\circ}-43303_{7 / 2}$ & & & \\
\hline 6426.638 & 20 & 15555.94 & $25656_{5 / 2}-41211^{\circ}{ }_{7 / 2}$ & & & & & 6300.742 & 1 & 15866.76 & $19132_{3 / 2}-34999^{\circ}{ }_{3 / 2}^{2}$ & & & \\
\hline 6425.366 & 4 & 15559.02 & & & & & & 6299.975 & $100 h$ & 15868.69 & $23873_{7 / 2}-39741_{9 / 2}^{\circ}$ & So & 1.03 & 0.095 \\
\hline 6424.452 & $6 h$ & 15561.23 & $22559^{\circ}{ }_{11 / 2}-38120_{9 / 2}$ & & & & & 6298.751 & 1 & 15871.77 & $23335^{\circ}{ }_{\tau / 2}-39206_{9 / 2}$ & & & \\
\hline 6421.975 & 1 & 15567.23 & $23309_{11 / 2}-38877^{\circ}{ }_{11 / 2}$ & & & & & 6298.268 & 1 & 15872.99 & $22791_{7 / 2}-38664^{\circ}{ }_{7 / 2}$ & & & \\
\hline 6420.390 & 5 & 15571.07 & $23873_{7 / 2}-39444_{9 / 2}^{\circ}$ & & & & & 6297.030 & 1 & 15876.11 & & & & \\
\hline 6416.319 & 60 & 15580.95 & $23781_{9 / 2}-39362^{\circ}{ }_{11 / 2}$ & so & 1.05 & 0.08 & & 6296.465 & 2 & 15877.54 & $25656_{s / 2}-41533_{s / 2}^{\circ}$ & & & \\
\hline 6412.770 & 10 & 15589.58 & $23873_{7 / 2}-39462^{\circ}{ }_{7 / 2}^{\circ}$ & & & & & 6295.570 & 1 & 15879.79 & & & & \\
\hline 6410.978 & 3 & 15593.93 & & & & & & 6294.959 & 3 & 15881.34 & $25207_{3 / 2}^{\circ}-41089_{1 / 2}$ & & & \\
\hline 6410.150 & 30 & 15595.95 & $18853_{11 / 2}-34449^{\circ}{ }_{13 / 2}$ & & & & & 6294.475 & 30 & 15882.56 & & so & 1.16 & 0.095 \\
\hline 6409.603 & 10 & 15597.28 & $23873_{7 / 2}-39470^{\circ}{ }_{5 / 2}$ & & & & & 6291.647 & 5 & 15889.70 & $21120_{7 / 2}-37010^{\circ}{ }_{3 / 2}$ & So & 0.84 & 0.27 \\
\hline 6409.479 & 10 & 15597.58 & $18990_{11 / 2}-34587_{9 / 2}^{\circ}$ & & & & & 6288.756 & 3 & 15897.00 & $17343_{\tau / 2}-33240^{\circ}$ & & & \\
\hline 6408.274 & $2 h$ & 15600.51 & $24246^{\circ}{ }^{\circ}-39847_{7 / 2}$ & & & & & 6288.335 & 2 & 15898.06 & $24957^{\circ}{ }_{11 / 2}-40855_{13 / 2}$ & & & \\
\hline 6406.823 & 50 & 15604.05 & $18853_{11 / 2}-34457^{\circ}{ }_{9 / 2}$ & so & 0.99 & 0.16 & & 6286.746 & 1 & 15902.08 & & & & \\
\hline 6405.582 & 3 & 15607.07 & $23873_{7 / 2}-39480^{\circ}{ }_{7 / 2}$ & & & & & 6285.873 & 1 & 15904.29 & & & & \\
\hline 6403.209 & 20 & 15612.85 & & & & & & 6285.205 & 2 & 15905.98 & $23941_{9 / 2}^{\circ}-39847_{\tau_{7 / 2}}$ & & & \\
\hline 6402.320 & 40 & 15615.02 & $23873_{\tau / 2}-39488_{9 / 2}^{\circ}$ & $\mathrm{T}$ & 1.09 & & & 6283.915 & 7 & 15909.25 & $25520^{\circ}{ }_{11 / 2}-41430_{11 / 2}$ & & & \\
\hline 6401.439 & 200 & 15617.17 & $16742_{\tau / 2}-32359^{\circ}{ }_{7 / 2}$ & $\Delta \mathrm{J}=0$ & 1. 32 & 1.19 & * & 6279.574 & 2 & 15920.24 & $21737_{8 / 2}-37657^{\circ}{ }_{11 / 2}^{/ 2}$ & & & \\
\hline 6398.121 & 5 & 15625.27 & $30972_{11 / 2}-46597^{\circ}{ }_{13 / 2}$ & & & & & 6278.563 & 1 & 15922.81 & $22419_{9 / 2}^{\circ}-38342_{7 / 2}$ & & & \\
\hline 6397.533 & 10 & 15626.71 & $17613_{9 / 2}-33240^{\circ}{ }_{7 / 2}$ & $\mathrm{~T}$ & 1.21 & & & 6277.767 & $5 h$ & 15924.83 & $23335^{\circ}{ }_{7 / 2}-39259_{s / 2}$ & & & \\
\hline 6396.134 & 2 & 15630.12 & $25520^{\circ}{ }_{11 / 2}-41151_{9 / 2}$ & & & & & 6277.515 & 50 & 15925.47 & $21799_{3 / 2}^{\circ / 2}-37724_{s / 2}^{\circ}$ & & & \\
\hline 6396.065 & 20 & 15630.29 & $27037^{\circ}{ }_{7 / 2}-42667_{9 / 2}$ & $\mathrm{~T}$ & 1.08 & & & 6276.349 & 6 & 15928.42 & $19753_{7 / 2}^{/ 2}-35682^{\circ}{ }_{7 / 2}$ & $\Delta \mathrm{J}=0$ & 1.14 & 0.23 \\
\hline 6389.319 & 10 & 15646.80 & $8771_{s / 2}^{\circ}-24418_{s / 2}$ & & & & & 6276.191 & 1 & 15928.83 & & & & \\
\hline 6388.534 & 5 & 15648.72 & $21997_{11 / 2}-37646^{\circ}{ }_{13 / 2}$ & & & & & 6275.622 & 7 & 15930.27 & & $\Delta \mathrm{J}=0$ & 1.00 & 0.44 \\
\hline 6386.912 & $6 w$ & 15652.69 & $22468^{\circ}{ }_{11 / 2}-38120_{9 / 2}$ & & & & & 6274.882 & $6 h$ & 15932.15 & $22929_{s / 2}-38861_{s / 2}^{\circ}$ & & & \\
\hline 6386.744 & 4 & 15653.10 & $25699_{9 / 2}^{\circ}-41352_{11 / 2}$ & & & & & 6274.417 & 10 & 15933.33 & $25536^{\circ}{ }_{15 / 2}-41469_{13 / 2}$ & $\mathrm{~T}$ & 1.20 & \\
\hline 6385.527 & 4 & 15656.09 & $16456_{17 / 2}-32112^{\circ}{ }_{15 / 2}$ & & & & & 6271.029 & 1 & 15941.94 & $22902^{\circ}{ }_{13 / 2}-38844_{11 / 2}$ & & & \\
\hline 6385.130 & 6 & 15657.06 & $25536^{\circ}{ }_{15 / 2}-41193_{13 / 2}$ & & & & & 6269.050 & 1 & 15946.97 & $27491_{11 / 2}-43438^{\circ}{ }_{13 / 2}$ & & & \\
\hline 6384.951 & 1 & 15657.50 & & & & & & 6263.850 & $20 h$ & 15960.21 & $23781_{9 / 2}-39741_{9 / 2}^{\circ}$ & $\Delta \mathrm{J}=0$ & 1.12 & 0.13 \\
\hline 6383.744 & 2 & 15660.46 & $21997_{11 / 2}-37657^{\circ}{ }_{11 / 2}$ & & & & & 6257.635 & 2 & 15976.06 & & & & \\
\hline 6380.270 & 200 & 15668.99 & $23873_{7 / 2}-39542^{\circ}{ }_{7 / 2}$ & $\Delta \mathrm{J}=0$ & 1.10 & 0.15 & & 6257.439 & 2 & 15976.56 & $24137_{13 / 2}-40113^{\circ}{ }_{13 / 2}$ & & & \\
\hline 6376.166 & 7 & 15679.07 & $18853_{11 / 2}-34532^{\circ}{ }_{11 / 2}$ & & & & & 6248.071 & 1 & 16000.51 & $25699_{9 / 2}^{\circ}-41700_{11 / 2}$ & & & \\
\hline 6375.383 & 40 & 15681.00 & $23781_{9 / 2}-39462^{\circ}{ }_{7 / 2}$ & $\mathrm{~T}$ & 1.08 & & & 6247.687 & 3 & 16001.50 & $23941_{9 / 2}^{\circ}-39942_{11 / 2}$ & & & \\
\hline 6371.152 & 1 & 15691.41 & $22791_{7 / 2}-38482^{\circ}{ }_{7 / 2}$ & & & & & 6243.947 & 1 & 16011.08 & & & & \\
\hline 6369.489 & 10 & 15695.51 & $18837_{9 / 2}-34532^{\circ}{ }_{11 / 2}^{1 / 2}$ & & & & & 6242.292 & 2 & 16015.33 & $21997_{11 / 2}-38012^{\circ}{ }_{13 / 2}$ & & & \\
\hline 6368.412 & 6 & 15698.16 & $23781_{9 / 2}-39479_{11 / 2}^{\circ}$ & & & & & 6241.869 & 1 & 16016.41 & & & & \\
\hline 6367.880 & 100 & 15699.47 & $16742_{7 / 2}-32441^{\circ}{ }_{9 / 2}$ & so & 0.99 & 0.25 & & 6237.245 & 1 & 16028.29 & $25699_{9 / 2}^{\circ}-41728_{9 / 2}$ & & & \\
\hline 6365.022 & 8 & 15706.52 & $23781_{9 / 2}-39488_{9 / 2}^{\circ}$ & & & & & 6225.129 & 1 & 16059.48 & $23374_{13 / 2}-39434^{\circ}{ }_{13 / 2}$ & & & \\
\hline 6364.489 & 1 & 15707.84 & $22791_{7 / 2}-38499^{\circ}$ & & & & & 6224.548 & 8 & 16060.98 & $26368_{11 / 2}^{\circ}-42429_{11 / 2}$ & $\Delta \mathrm{J}=0$ & 1.14 & 0.33 \\
\hline 6364.323 & 1 & 15708.25 & $28555_{\tau / 2}-44263^{\circ}{ }_{7 / 2}$ & & & & & 6223.272 & 5 & 16064.27 & $18990_{11 / 2}-35054_{9 / 2}^{\circ}$ & So & 0.90 & 0.30 \\
\hline 6362.908 & 10 & 15711.74 & $26368^{\circ}{ }_{11 / 2}^{2}-42080_{11 / 2}$ & & & & & 6220.419 & 1 & 16071.64 & $26357^{\circ}{ }_{13 / 2}-42429_{11 / 2}$ & & & \\
\hline 6359.156 & 3 & 15721.01 & & & & & & 6216.288 & 3 & 16082.32 & $24611_{s / 2}^{\circ}-40693_{3 / 2}$ & & & \\
\hline 6358.588 & 4 & 15722.42 & $26357^{\circ}{ }_{13 / 2}-42080_{11 / 2}$ & & & & & " & & & $22419_{9 / 2}^{\circ}-38502_{9 / 2}$ & & & \\
\hline
\end{tabular}


APPENDIX - Spectral Lines of Neutral Thulium-Continued

\begin{tabular}{|c|c|c|c|c|c|c|c|c|c|c|c|c|c|}
\hline \multirow{2}{*}{$\begin{array}{c}\text { Wave- } \\
\text { length } \\
\text { in air } \\
\AA\end{array}$} & \multirow[t]{2}{*}{ Intensity } & \multirow{2}{*}{$\begin{array}{l}\text { Wave- } \\
\text { number } \\
\mathrm{cm}^{-1}\end{array}$} & \multirow[t]{2}{*}{ Classification } & \multirow{2}{*}{\multicolumn{3}{|c|}{$\begin{array}{l}\text { Zeeman effect } \\
\text { e f } \quad \text { Je }\end{array}$}} & \multirow{2}{*}{$\begin{array}{c}\text { Wave- } \\
\text { length } \\
\text { in air } \\
\AA\end{array}$} & \multirow[t]{2}{*}{ Intensity } & $\begin{array}{l}\text { Wave- } \\
\text { number }\end{array}$ & Classification & & eeman & ffect \\
\hline & & & & & & & & & $\mathrm{cm}^{-1}$ & & type & f & Je \\
\hline 6214.069 & 3 & 16088.06 & $26368^{\circ}{ }_{11 / 2}-42456_{13 / 2}$ & & & & 6075.211 & 1 & 16455.78 & & & & \\
\hline 6211.445 & 40 & 16094.86 & $26448_{s / 2}^{\circ}-42543_{7 / 2}$ & $\mathrm{~T}$ & 1.06 & & 6074.670 & 1 & 16457.24 & $22929_{\mathrm{s} / 2}-39386_{\mathrm{s} / 2}^{\circ}$ & & & \\
\hline 6210.814 & 1 & 16096.50 & $25745_{\mathrm{s} / 2}-41841^{\circ / 2}{ }_{7 / 2}$ & & & & 6072.656 & 2 & 16462.70 & $25717_{7 / 2}-42179^{\circ}{ }_{s / 2}$ & & & \\
\hline 6209.952 & 10 & 16098.73 & $26357^{\circ}{ }_{13 / 2}-42456_{13 / 2}$ & $\Delta \mathrm{J}=0$ & 1.20 & 0.08 & 6068.945 & 1 & 16472.77 & $24957^{\circ}{ }_{11 / 2}-41430_{11 / 2}$ & & & \\
\hline 6209.215 & 7 & 16100.64 & $21120_{7 / 2}-37221^{\circ}{ }_{7 / 2}$ & & & & 6068.508 & 1 & 16473.95 & $21737_{\mathrm{g} / 2}-38211^{\circ}{ }_{7 / 2}$ & & & \\
\hline 6204.446 & 5 & 16113.02 & $17613_{9 / 2}-33726_{9 / 2}^{\circ}$ & & & & 6067.411 & 10 & 16476.93 & $27037^{\circ}{ }_{7 / 2}-43514_{\tau^{\prime} / 2}$ & & & \\
\hline 6204.082 & 1 & 16113.96 & & & & & 6064.827 & 1 & 16483.95 & $22929_{s_{s / 2}}-39413_{7_{/ 2}}^{\circ}$ & & & \\
\hline 6203.927 & 20 & 16114.36 & $16742_{\tau / 2}-32856^{\circ}{ }_{s / 2}$ & SI & 1.33 & $1.06 *$ & 6063.169 & 20 & 16488.46 & $17454_{1: 1 / 2}-33943^{\circ}{ }_{1: / 2}^{13 / 2}$ & & & \\
\hline 6200.154 & 1 & 16124.17 & $23309_{11 / 2}-39434_{13 / 2}^{\circ}$ & & & & 6062.533 & 1 & 16490.19 & $22929_{\mathrm{s} / 2}-39419^{\circ}: 1 / 2$ & & & \\
\hline 6199.916 & 1 & 16124.79 & $19548_{5 / 2}-35673_{5 / 2}^{\circ}$ & & & & 6059.091 & 2 & 16499.56 & & & & \\
\hline 6197.110 & 10 & 16132.09 & $22929_{5 / 2}-39061_{3 / 2}^{\circ}$ & & & & 6058.988 & 5 & 16499.84 & $26368^{\circ}{ }_{11 / 2}-42868_{11 / 2}$ & & & \\
\hline 6196.602 & 4 & 16133.41 & $19548_{5 / 2}-35682^{\circ}{ }_{7 / 2}$ & & & & 6057.992 & 1 & 16502.55 & & & & \\
\hline 6190.922 & 4 & 16148.22 & $26448_{s / 2}^{\circ}-42596_{s / 2}$ & & & & 6057.553 & 2 & 16503.75 & $26448_{s / 2}^{\circ}-42952_{7 / 2}$ & & & \\
\hline 6184.958 & 2 & 16163.79 & $21997_{11 / 2}-38161^{\circ}{ }_{11 / 2}^{\circ}$ & & & & 6055.655 & 2 & 16508.92 & & & & \\
\hline 6180.164 & 10 & 16176.32 & & & & & 6055.268 & 1 & 16509.97 & $18853_{11 / 2}-35363_{9 / 2}^{\circ}$ & & & \\
\hline 6180.011 & 1 & 16176.73 & $26368^{\circ}{ }_{11 / 2}-42545_{13 / 2}$ & & & & 6055.072 & 2 & 16510.51 & $26357^{\circ}{ }_{1: 1 / 2}-42868_{11 / 2}$ & & & \\
\hline $\begin{array}{l}6179.429 \\
, "\end{array}$ & 1 & 16178.25 & $\begin{array}{l}23309_{11 / 2}-39488_{9 / 2}^{\circ} \\
24418_{5 / 2}^{\circ}-40596^{\circ}{ }_{7 / 2}^{\circ}\end{array}$ & & & & $\begin{array}{c}6054.541 \\
, "\end{array}$ & 20 & 16511.96 & $\begin{array}{l}23335^{\circ}{ }_{7 / 2}-39847_{7 / 2} \\
24957^{\circ}{ }_{11 / 2}-41469_{1: 3 / 2}\end{array}$ & & & \\
\hline 6179.047 & 10 & 16179.25 & $25520^{\circ}{ }_{11 / 2}-41700_{11 / 2}^{1 / 2}$ & $\Delta \mathrm{J}=0$ & 1.14 & 0.16 & 6052.810 & 1 & 16516.68 & & & & \\
\hline 6178.956 & 1 & 16179.49 & $17613_{9 / 2}-33793_{11 / 2}^{\circ}$ & & & & 6049.419 & 1 & 16525.94 & $24137_{1: 1 / 2}-40663^{\circ}{ }_{11 / 2}$ & & & \\
\hline 6177.259 & 30 & 16183.93 & $17454_{13 / 2}-33638^{\circ}{ }_{11 / 2}$ & S00 & 1.15 & $1.42 *$ & 6047.127 & 2 & 16532.20 & $21997_{11 / 2}-38529^{\circ}{ }_{11 / 2}$ & & & \\
\hline 6175.947 & $1 h$ & 16187.37 & $26357^{\circ}{ }_{13 / 2}-42545_{13 / 2}$ & & & & 6046.804 & 1 & 16533.08 & $22929_{s / 2}-39462^{\circ}{ }^{\circ} / 2$ & & & \\
\hline 6175.294 & 400 & 16189.08 & $13119_{9 / 2}-29308_{9 / 2}^{\circ}$ & $\Delta \mathrm{J}=0$ & 1.40 & 0.80 & 6044.181 & 10 & 16540.26 & $27440^{\circ}{ }^{\circ / 2}-43981_{\tau / 2}$ & & & \\
\hline 6170.806 & 8 & 16200.86 & $18853_{11 / 2}^{/ 2}-35054_{9 / 2}^{\circ / 2}$ & So & 0.55 & 0.60 & & & & $26889_{9 / 2}^{1 / 2}-43429^{\circ}{ }_{9 / 2}$ & & & \\
\hline 6169.821 & 1 & 16203.44 & & & & & 6043.989 & 2 & 16540.78 & $22929_{\mathrm{s} / 2}-39470_{\mathrm{s} / 2}^{\circ}$ & & & \\
\hline 6168.458 & 10 & 16207.02 & $25520_{11 / 2}^{\circ}-41728_{9 / 2}$ & SI & 1.32 & 0.20 & & & & $24246^{\circ}{ }_{7 / 2}-40787_{s / 2}$ & & & \\
\hline 6167.917 & 40 & 16208.44 & $17752_{\mathrm{s} / 2}-33961^{\circ}{ }_{7 / 2}$ & & & & 6043.767 & 2 & 16541.39 & $19132_{: 3 / 2}-35673^{\circ / 2}$ & & & \\
\hline 6166.500 & 20 & 16212.17 & $23335^{\circ}{ }_{7 / 2}-39547_{5 / 2}^{1 / 2}$ & SI & 1.55 & 0.19 & 6043.068 & 10 & 16543.31 & $25207^{\circ}: 1 / 2-4175 l_{5 / 2}$ & so & 1.06 & 1.36 \\
\hline 6164.550 & 2 & 16217.30 & $18837_{9 / 2}-35054_{9 / 2}^{\circ}$ & & & & 6040.260 & 1 & 16551.00 & $28340^{\circ}{ }_{7 / 2}^{\prime}-44891_{7 / 2}$ & & & \\
\hline 6160.720 & 40 & 16227.38 & $26368^{\circ}{ }_{11 / 2}-42595_{11 / 2}$ & & & & 6039.780 & 40 & 16552.31 & & & & \\
\hline 6156.671 & 10 & 16238.05 & $26357^{\circ}{ }_{13 / 2}-42595_{11 / 2}$ & & & & 6038.380 & 2 & 16556.15 & $24246^{\circ}{ }_{7 / 2}-40802_{7 / 2}$ & & & \\
\hline 6153.396 & 1 & 16246.69 & & & & & 6037.696 & 1 & 16558.02 & & & & \\
\hline 6151.992 & 1 & 16250.40 & & & & & 6037.279 & 15 & 16559.17 & $25520^{\circ}{ }_{11 / 2}-42080_{11 / 2}$ & & & \\
\hline 6151.000 & 5 & 16253.02 & $23309_{11 / 2}-39563^{\circ}{ }_{11 / 2}$ & & & & 6036.983 & 10 & 16559.98 & & & & \\
\hline 6146.215 & 2 & 16265.67 & $27037^{\circ}{ }_{7 / 2}-43303_{7 / 2}$ & & & & 6035.639 & 1 & 16563.67 & $22559^{\circ}{ }_{11 / 2}-39123_{9 / 2}$ & & & \\
\hline 6144.742 & 3 & 16269.57 & & & & & 6035.389 & 3 & 16564.35 & $23431_{s / 2}^{\circ}-39996_{z^{2} / 2}$ & & & \\
\hline 6142.800 & 5 & 16274.72 & $27440_{9 / 2}^{\circ}-43715_{11 / 2}$ & & & & 6034.254 & 1 & 16567.47 & & & & \\
\hline 6139.548 & 1 & 16283.34 & $16957_{7 / 2}^{1 / 2}-33240^{\circ}{ }_{7 / 2}^{1 / 2}$ & & & & 6031.303 & 3 & 16575.58 & $23941_{y / 2}^{\circ}-40516_{y / 2}$ & & & \\
\hline 6139.086 & 1 & 16284.56 & $22559^{\circ}{ }_{11 / 2}-38844_{11 / 2}$ & & & & 6026.521 & $1 h$ & 16588.73 & $26368^{\circ}{ }_{11 / 2}-42957_{11 / 2}$ & & & \\
\hline 6138.795 & 10 & 16285.34 & $25207_{3 / 2}^{\circ}-41493_{5 / 2}$ & & & & 6025.437 & 200 & 16591.71 & $17454_{1: 1 / 2}-34046_{15 / 2}^{\circ}$ & SI & 1.45 & 0.30 \\
\hline 6131.532 & 200 & 16304.63 & $16456_{17 / 2}-32761^{\circ}{ }_{17 / 2}$ & $\Delta \mathrm{J}=0$ & 1.14 & 0.54 & 6024.192 & 1 & 16595.14 & & & & \\
\hline 6129.673 & 1 & 16309.57 & & & & & 6021.645 & 2 & 16602.16 & $27377_{1: 1 / 2}-43979^{\circ}{ }_{1: 1 / 2}$ & & & \\
\hline 6119.445 & 2 & 16336.83 & $25207_{3 / 2}^{\circ}-41544_{s_{/ 2}}$ & & & & 6020.036 & 2 & 16606.60 & & & & \\
\hline 6118.883 & 150 & 16338.33 & $17454_{13 / 2}^{1 / 2}-33793^{\circ}{ }_{11 / 2}$ & so & 0.65 & 0.50 & 6019.165 & 1 & 16609.00 & & & & \\
\hline 6117.028 & 2 & 16343.28 & & & & & 6018.978 & 1 & 16609.52 & & & & \\
\hline 6115.490 & $5 h$ & 16347.39 & $17613_{9 / 2}-33961^{\circ}{ }_{7 / 2}$ & & & & 6018.732 & 1 & 16610.20 & $21737_{9 / 2}-38347^{\circ}{ }_{11 / 2}$ & & & \\
\hline 6114.360 & 1 & 16350.42 & $21997_{11 / 2}-38347^{\circ}{ }_{11 / 2}^{\circ}$ & & & & 6017.905 & 2 & 16612.48 & $22929_{s / 2}-39542_{7 / 2}^{\circ}$ & & & \\
\hline 6111.778 & 5 & 16357.32 & $17454_{13 / 2}-33812^{\circ}{ }_{13 / 2}$ & & & & 6017.724 & 2 & 16612.98 & $17752_{s / 2}-34365_{7 / 2}^{\circ / 2}$ & & & \\
\hline 6109.439 & 1 & 16363.59 & $23335_{7 / 2}^{\circ / 2}-39698_{9 / 2}$ & & & & 6017.195 & 1 & 16614.44 & & & & \\
\hline 6104.797 & 2 & 16376.03 & $22468^{\circ}{ }_{11 / 2}^{2}-38844_{11 / 2}$ & & & & 6016.016 & 7 & 16617.69 & $17343_{\tau / 2}-33961^{\circ}{ }_{\tau / 2}$ & & & \\
\hline 6103.150 & 5 & 16380.45 & $25699_{9 / 2}^{\circ}-42080_{11 / 2}$ & & & & 6015.899 & 5 & 16618.02 & $25520_{11 / 2}^{\circ}-42139_{9 / 2}$ & & & \\
\hline 6101.149 & 1 & 16385.82 & $25488_{11 / 2}^{8 / 2}-41874_{11 / 2}^{\circ}$ & & & & 6014.283 & 1 & 16622.48 & $22791_{7 / 2}-39413^{\circ}{ }_{7 / 2}^{1 / 2}$ & & & \\
\hline 6100.538 & 3 & 16387.46 & $21997_{11 / 2}-38384^{\circ / 2}$ & & & & 6013.767 & 1 & 16623.91 & $19753_{7 / 2}-36377_{9 / 2}^{\circ}$ & & & \\
\hline 6096.377 & 1 & 16398.65 & $21120_{\tau / 2}-37519_{\tau / 2}^{\circ}$ & & & & 6013.417 & 1 & 16624.88 & $26488_{: 1 / 2}^{/ 2}-43113^{\circ}{ }_{s / 2}^{1 / 2}$ & & & \\
\hline 6093.856 & 1 & 16405.43 & $29308_{9 / 2}^{\circ}-45714_{11 / 2}$ & & & & 6011.856 & 3 & 16629.19 & $19748_{9 / 2}-36377_{9 / 2}^{\circ}$ & & & \\
\hline 6092.771 & 1 & 16408.35 & & & & & 6011.777 & 8 & 16629.41 & $15587_{11 / 2}-32217^{\circ}{ }^{\circ / 2}$ & & & \\
\hline 6091.873 & 1 & 16410.77 & & & & & 6011.373 & 1 & 16630.53 & $25488_{11 / 2}-42119^{\circ}{ }_{11 / 2}$ & & & \\
\hline 6090.220 & 2 & 16415.22 & $24348_{9 / 2}-40763_{7 / 2}^{\circ}$ & & & & 6010.058 & 3 & 16634.17 & & & & \\
\hline & & & $23431_{s / 2}^{\circ}-39847_{\tau^{2} / 2}^{1 / 2}$ & & & & 6009.920 & 15 & 16634.55 & $21799_{3 / 2}^{\circ}-38433_{s / 2}$ & & & \\
\hline 6088.713 & 1 & 16419.29 & & & & & 6009.812 & 2 & 16634.85 & $23431_{s / 2}^{\circ}-40066_{s / 2}^{\circ}$ & & & \\
\hline 6086.848 & 8 & 16424.32 & $22419^{\circ}{ }_{9 / 2}-38844_{11 / 2}$ & & & & 6009.693 & 1 & 16635.18 & & & & \\
\hline 6085.977 & 2 & 16426.67 & $27037^{\circ}{ }_{7 / 2}^{1 / 2}-43464_{y / 2}$ & & & & 6009.191 & 1 & 16636.57 & & & & \\
\hline 6085.445 & 1 & 16428.10 & $26448_{s / 2}^{\circ}-42876_{s / 2}^{\circ}$ & & & & 6008.262 & 5 & 16639.14 & & & & \\
\hline 6084.574 & 1 & 16430.46 & & & & & 6006.610 & 1 & 16643.72 & $24137_{13 / 2}-40780^{\circ}{ }_{13 / 2}$ & & & \\
\hline 6082.898 & 1 & 16434.98 & $17343_{7 / 2}-33778_{7 / 2}^{\circ}$ & & & & 6005.338 & 3 & 16647.24 & $21737_{9 / 2}-38384_{9 / 2}^{\circ}$ & & & \\
\hline 6081.586 & 1 & 16438.53 & $21799^{\circ}: / 2-38237_{: / 2}^{\prime 2}$ & & & & 6005.315 & 1 & 16647.31 & $22559^{\circ}{ }_{11 / 2}-39206_{9 / 2}$ & & & \\
\hline 6080.782 & 1 & 16440.70 & $32107^{\circ}{ }_{11 / 2}-48547_{9 / 2}^{1 / 2}$ & & & & 6004.191 & l & 16650.42 & $25717_{\gamma_{/ 2}}-42367^{\circ}{ }_{s / 2}$ & & & \\
\hline 6080.504 & 10 & 16441.45 & $17752_{\mathrm{s} / 2}-34194_{\mathrm{s} / 2}^{\circ}$ & & & & 6003.711 & 3 & 16651.75 & $24701^{\circ}{ }^{\circ}-41352_{11 / 2}$ & & & \\
\hline 6078.362 & 2 & 16447.25 & & & & & 6003.222 & 1 & 16653.11 & $22791_{\gamma_{/ 2}}-39444_{9 / 2}^{\circ / 2}$ & & & \\
\hline 6075.555 & 4 & 16454.85 & & & & & 6002.998 & 1 & 16653.73 & $16742_{7 / 2}-33396^{\circ}{ }_{7 / 2}$ & & & \\
\hline
\end{tabular}


APPENDIX - Spectral Lines of Neutral Thulium - Continued

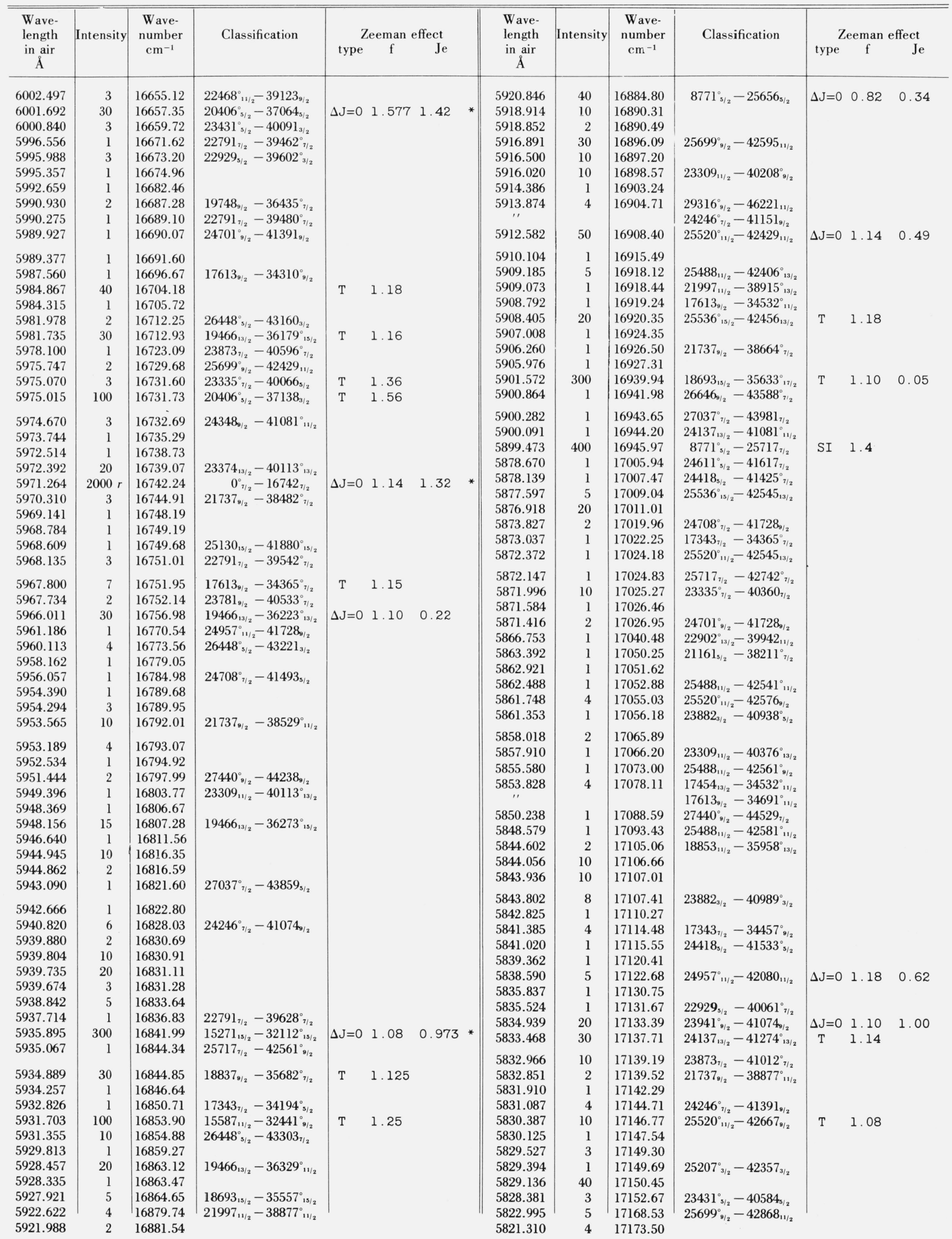


APPENDIX - Spectral Lines of Neutral Thulium - Continued

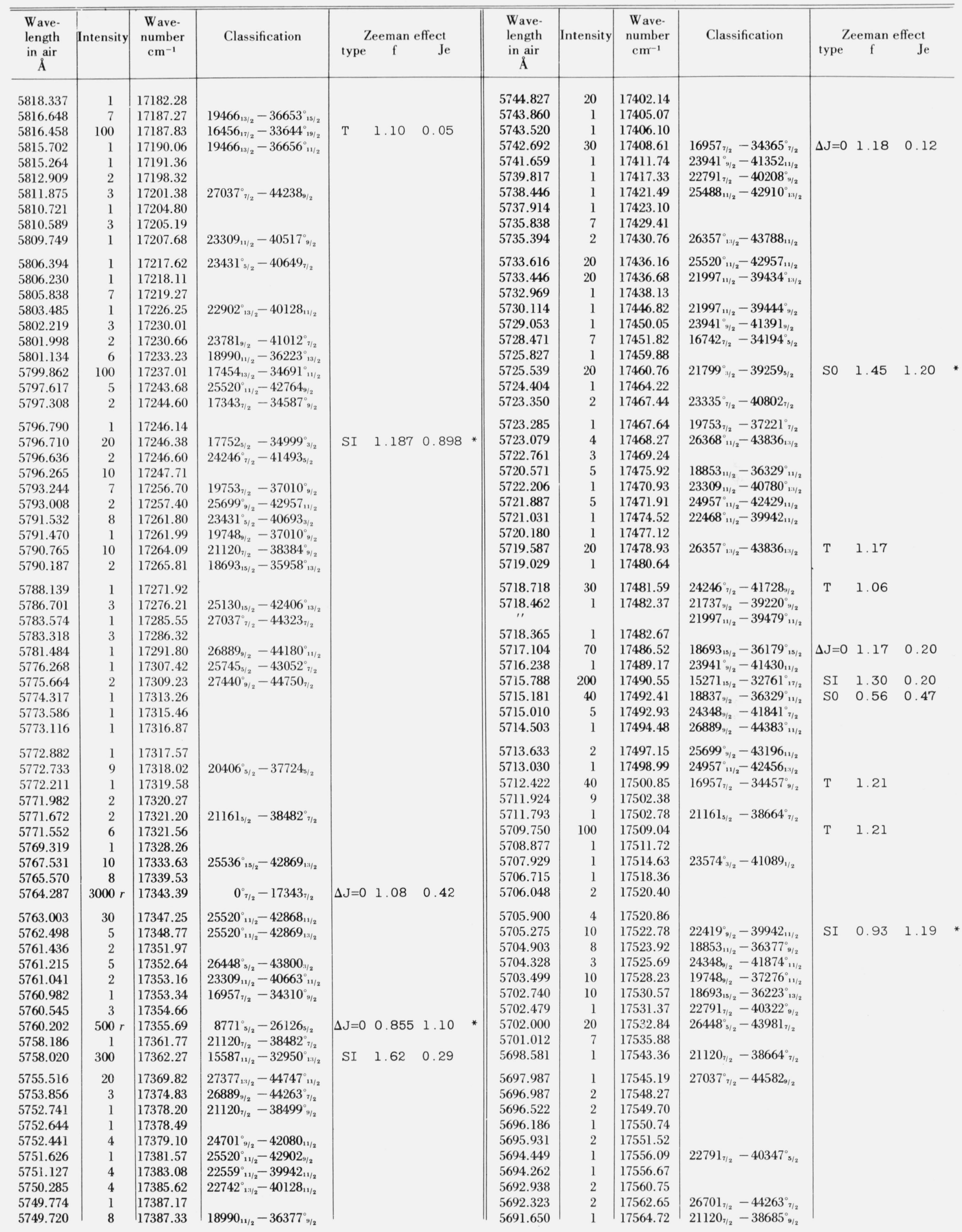


APPENDIX - Spectral Lines of Neutral Thulium - Continued

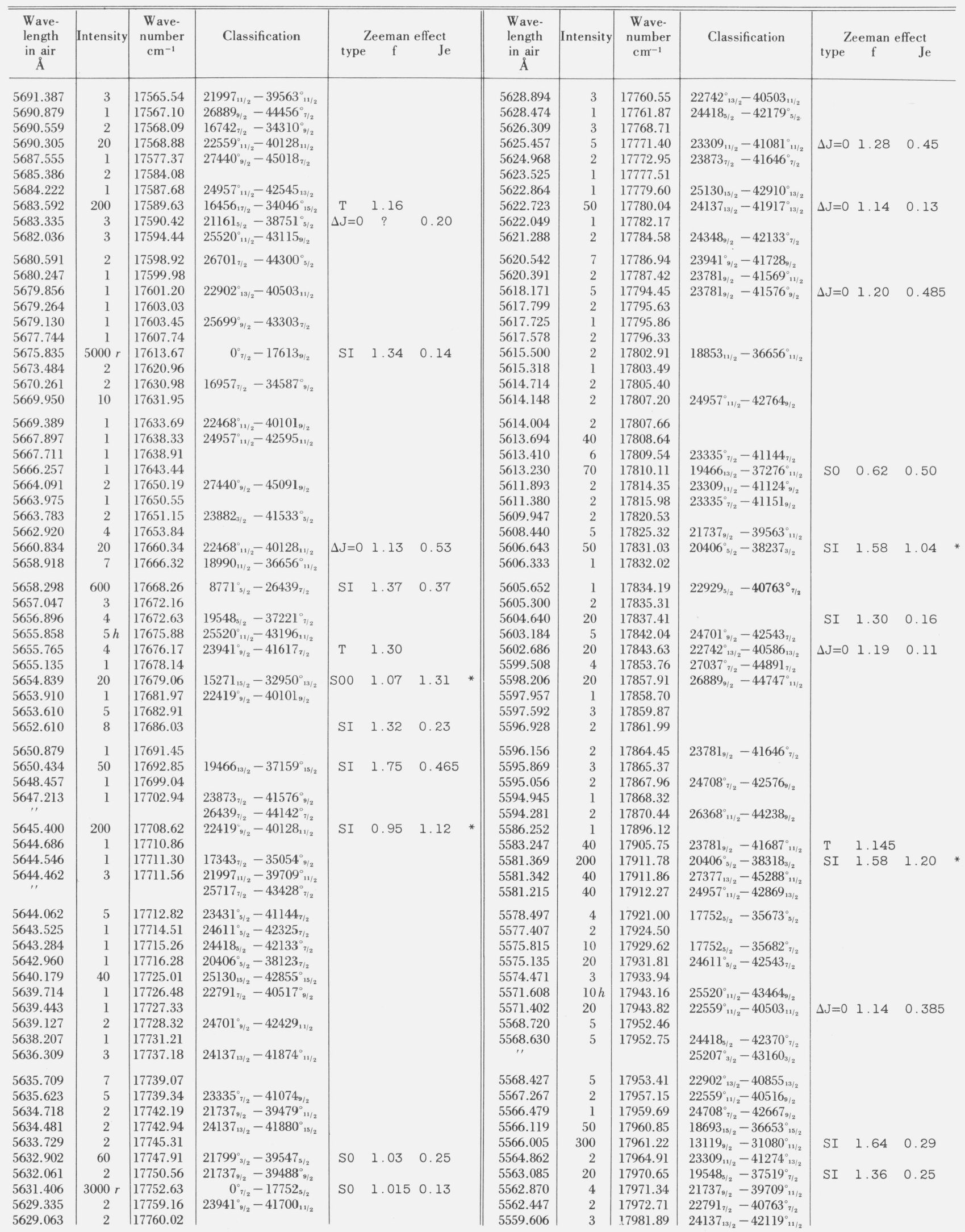


APPENDIX - Spectral Lines of Neutral Thulium-Continued

\begin{tabular}{|c|c|c|c|c|c|c|c|c|c|c|c|c|c|}
\hline \multirow{2}{*}{$\begin{array}{c}\text { Wave- } \\
\text { length } \\
\text { in air } \\
\AA\end{array}$} & \multirow{2}{*}{ Intensity } & \multirow{2}{*}{$\begin{array}{c}\text { W ave- } \\
\text { number } \\
\mathrm{cm}^{-1}\end{array}$} & \multirow[t]{2}{*}{ Classification } & \multirow{2}{*}{\multicolumn{3}{|c|}{ Zeeman effect }} & \multirow{2}{*}{$\begin{array}{c}\text { Wave- } \\
\text { length } \\
\text { in air } \\
\AA\end{array}$} & \multirow[t]{2}{*}{ Intensity } & $\begin{array}{c}\text { Wave- } \\
\text { number }\end{array}$ & Classification & & eeman & ffect \\
\hline & & & & & & & & & & & type & $\mathrm{f}$ & $\mathrm{Je}$ \\
\hline 5556.248 & 3 & 17992.76 & & & & & 5482.945 & 5 & 18233.31 & $24348_{9 / 2}-42581_{11 / 2}^{\circ}$ & SI & 1.26 & 0.11 \\
\hline 5554.115 & 20 & 17999.67 & $24957^{\circ}{ }_{11 / 2}-42957_{11 / 2}$ & & & & 5482.533 & 2 & 18234.68 & $25520^{\circ}{ }_{11 / 2}-43755_{9 / 2}$ & & & \\
\hline 5551.642 & 5 & 18007.69 & & & & & 5481.113 & 3 & 18239.40 & $24957^{\circ}{ }_{11 / 2}-43196_{11 / 2}$ & & & \\
\hline 5551.385 & 1 & 18008.52 & $23873_{7 / 2}-41881_{9 / 2}^{\circ}$ & & & & 5481.054 & 10 & 18239.60 & & & & \\
\hline 5550.802 & 3 & 18010.41 & $24708^{\circ}{ }_{7 / 2}^{\circ}-42718_{9 / 2}$ & & & & 5479.724 & 3 & 18244.03 & $24708^{\circ}{ }_{7 / 2}-42952_{7 / 2}$ & & & \\
\hline 5549.665 & 20 & 18014.10 & $25207_{3 / 2}^{\circ}-4322 l_{3 / 2}$ & & & & 5478.498 & 80 & 18248.11 & $13119_{9 / 2}-31367^{\circ}{ }_{7 / 2}$ & $\mathrm{~T}$ & 1.25 & 0.06 \\
\hline 5549.442 & 10 & 18014.83 & $19753_{7 / 2}-37768_{9 / 2}$ & & & & 5476.100 & 20 & 18256.10 & $24701_{9 / 2}^{\circ}-42957_{11 / 2}$ & & & \\
\hline 5549.121 & 6 & 18015.87 & $25699_{9 / 2}^{\circ}-43715_{11 / 2}^{\circ}$ & & & & 5474.455 & 20 & 18261.58 & $23574_{3 / 2}^{\circ}-41836_{5 / 2}$ & & & \\
\hline 5548.787 & 3 & 18016.95 & & & & & 5473.079 & 20 & 18266.18 & $23309_{11 / 2}-41576_{9 / 2}^{\circ}$ & & & \\
\hline 5548.650 & 4 & 18017.40 & $24701_{9 / 2}^{\circ}-42718_{9 / 2}$ & & & & 5472.710 & 40 & 18267.41 & $25520^{\circ}{ }_{11 / 2}-43788_{11 / 2}$ & & & \\
\hline $\begin{array}{c}5547.813 \\
, \prime\end{array}$ & 10 & 18020.12 & $\begin{array}{l}18990_{11 / 2}-37010_{9 / 2}^{\circ} \\
19748_{9 / 2}-37768_{9 / 2}^{\circ}\end{array}$ & & & & $\begin{array}{l}5472.363 \\
5470.913\end{array}$ & $\begin{array}{r}4 \\
10\end{array}$ & $\begin{array}{l}18268.57 \\
18273.41\end{array}$ & & & & \\
\hline 5547.230 & 1 & 18022.01 & $24348_{9 / 2}-42370^{\circ}{ }_{7 / 2}^{/ 2}$ & & & & 5468.303 & 10 & 18282.13 & $23335^{\circ}{ }_{\gamma_{/ 2}}-41617_{\tau / 2}$ & & & \\
\hline 5545.666 & 20 & 18027.09 & $20406_{5 / 2}^{\circ}-38433_{5 / 2}^{1 / 2}$ & & & & 5466.596 & 2 & 18287.84 & $24418_{\mathrm{s} / 2}^{1 / 2}-42705_{\mathrm{s} / 2}^{\circ / 2}$ & & & \\
\hline 5545.312 & 5 & 18028.24 & & & & & 5465.641 & 3 & 18291.03 & $22902^{\circ}{ }_{13 / 2}-41193_{13 / 2}$ & & & \\
\hline 5544.968 & 8 & 18029.36 & & & & & 5465.362 & 3 & 18291.97 & & & & \\
\hline 5544.613 & 1 & 18030.52 & & & & & 5465.299 & 10 & 18292.18 & $21799_{3 / 2}^{\circ}-40091_{3 / 2}$ & $\Delta \mathrm{J}=0$ & 1.47 & 1.15 \\
\hline 5543.148 & 4 & 18035.28 & $22468^{\circ}{ }_{11 / 2}-40503_{11 / 2}$ & & & & 5465.103 & 4 & 18292.83 & $21120_{7 / 2}-39413_{7 / 2}^{\circ}$ & & & \\
\hline 5541.949 & 15 & 18039.18 & & & & & 5464.727 & 2 & 18294.09 & & & & \\
\hline 5539.907 & 1 & 18045.83 & & & & & 5464.621 & 1 & 18294.45 & & & & \\
\hline 5539.260 & 1 & 18047.94 & & & & & 5464.145 & 60 & 18296.04 & $22559^{\circ}{ }_{11 / 2}-40855_{13 / 2}$ & $\mathrm{~T}$ & 1.11 & 0.05 \\
\hline 5538.341 & 30 & 18050.94 & $15587_{11 / 2}-33638_{11 / 2}^{\circ}$ & $\Delta \mathrm{J}=0$ & 1.30 & 0.80 & 5463.723 & 1 & 18297.45 & $23882_{3 / 2}-42179^{\circ}{ }_{5 / 2}$ & & & \\
\hline 5537.535 & 3 & 18053.56 & $27037^{\circ}{ }_{7 / 2}-45091_{9 / 2}$ & & & & 5462.796 & 20 & 18300.56 & $25536^{\circ}{ }_{15 / 2}-43836_{13 / 2}$ & $\mathrm{~T}$ & 1.23 & \\
\hline 5536.785 & 10 & 18056.01 & $25488_{11 / 2}-43544_{9 / 2}$ & & & & 5462.428 & 5 & 18301.79 & $26448_{s / 2}^{\circ}-44750_{7 / 2}$ & & & \\
\hline " & & & $23335^{\circ}{ }_{7 / 2}-41391_{9 / 2}$ & & & & $\begin{array}{l}5460.245 \\
5455813\end{array}$ & 2 & $\begin{array}{l}18309.11 \\
18323.98\end{array}$ & $21161_{5 / 2}-39470^{\circ}{ }_{5 / 2}$ & & & \\
\hline 5535.507 & $\begin{array}{r}3 \\
30\end{array}$ & & $23431^{\circ}-41493$ & & & & $\begin{array}{l}5455.813 \\
5451.825\end{array}$ & $\begin{array}{l}1 \\
3\end{array}$ & $\begin{array}{l}18323.98 \\
18337.39\end{array}$ & $\begin{array}{l}24418_{5 / 2}-42742^{\circ}{ }^{\circ / 2} \\
23781_{9 / 2}-42119^{\circ}\end{array}$ & & & \\
\hline 5535.195 & 30 & $\begin{array}{l}18061.19 \\
18070.64\end{array}$ & $\begin{array}{l}23431_{5 / 2}-41493_{5 / 2} \\
26448_{5 / 2}^{\circ}-44518_{5 / 2}^{\circ}\end{array}$ & & & & $\begin{array}{l}5451.825 \\
5451.388\end{array}$ & $\begin{array}{l}3 \\
3\end{array}$ & $\begin{array}{l}18337.39 \\
18338.86\end{array}$ & $\begin{array}{l}23181_{9 / 2}-42119_{11 / 2}^{1 / 2} \\
17343_{7}-35682^{\circ}{ }_{712}\end{array}$ & & & \\
\hline 5532.301 & $\begin{array}{l}2 \\
3\end{array}$ & $\begin{array}{l}18070.64 \\
18072.93\end{array}$ & $\begin{array}{l}26448_{\mathrm{s} / 2}-44518_{5 / 2} \\
26701_{7 / 2}-44774_{9 / 2}^{\circ}\end{array}$ & & & & 5449.480 & $\begin{array}{l}3 \\
2\end{array}$ & $\begin{array}{l}18338.80 \\
18345.28\end{array}$ & $17343_{7 / 2}-35682_{7 / 2}$ & & & \\
\hline $\begin{array}{l}5531.600 \\
5529.083\end{array}$ & $\begin{array}{l}3 \\
2\end{array}$ & $\begin{array}{l}18072.93 \\
18081.16\end{array}$ & $26448^{\circ}{ }_{5 / 2}^{\circ}-44529_{7 / 2}^{9 / 2}$ & & & & 5642.596 & 300 & 17717.42 & $8771_{3 / 2}^{\circ}-26488_{3 / 2}$ & $\mathrm{~T}$ & 0.85 & \\
\hline 5528.702 & 2 & 18082.41 & $24701_{9 / 2}^{\circ}-42783_{7 / 2}$ & & & & 5641.273 & 15 & 17721.58 & $20406_{5 / 2}^{\circ}-38128_{s / 2}$ & $\Delta \mathrm{J}=0$ & 1.58 & 1.05 \\
\hline 5528.343 & 200 & 18083.58 & $22419_{9 / 2}^{\circ / 2}-40503_{11 / 2}^{\circ}$ & SI & 0.935 & $1.105 *$ & 5448.178 & 2 & 18349.66 & $21120_{7 / 2}-39470^{\circ}{ }_{5 / 2}^{2}$ & & & \\
\hline 5526.614 & 2 & 18089.24 & & & & & 5446.453 & 60 & 18355.47 & $15587_{11 / 2}-33943^{\circ}{ }_{13 / 2}$ & so & 0.45 & 0.67 \\
\hline 5525.560 & 10 & 18092.69 & $23781_{9 / 2}-41874_{11 / 2}^{\circ}$ & & & & 5440.751 & 6 & 18374.71 & $18990_{11 / 2}-37365_{13 / 2}^{\circ}$ & & & \\
\hline 5524.271 & 4 & 18096.91 & & & & & 5440.438 & 1 & 18375.77 & & & & \\
\hline 5524.034 & 3 & 18097.69 & $16957_{7 / 2}-35054_{9 / 2}^{\circ}$ & & & & 5439.559 & 3 & 18378.74 & $21997_{11 / 2}-40376^{\circ}{ }_{13 / 2}$ & & & \\
\hline & & & $26448_{5 / 2}-44545_{3 / 2}^{\circ}$ & & & & 5436.972 & 50 & 18387.48 & $22468^{\circ}{ }_{11 / 2}-40855_{13 / 2}$ & so & 0.94 & 0.22 \\
\hline 5522.451 & 1 & 18102.87 & $17454_{13 / 2}-35557^{\circ}{ }_{15 / 2}$ & & & & 5435.645 & 20 & 18391.97 & $19466_{13 / 2}-37858_{15 / 2}^{\circ}$ & SI & 1.48 & 0.27 \\
\hline 5520.169 & 1 & 18110.36 & & & & & 5434.171 & 6 & 18396.96 & & & & \\
\hline $\begin{array}{c}5519.440 \\
, "\end{array}$ & 1 & 18112.75 & $23431^{\circ}{ }_{5 / 2}-41544_{5 / 2}$ & & & & 5433.334 & 5 & 18399.79 & & & & \\
\hline & & & $22742_{13 / 2}^{\circ}-40855_{13 / 2}$ & & & & 5431.098 & 3 & 18407.37 & $24708^{\circ}{ }_{\tau / 2}-43115_{9 / 2}$ & & & \\
\hline 5518.545 & 4 & 18115.69 & $21161_{5 / 2}-39277_{5 / 2}^{\circ}$ & & & & 5426.505 & 10 & 18422.95 & $18853_{11 / 2}-37276^{\circ}$ & $\Delta \mathrm{J}=0$ & 1.19 & 0.61 \\
\hline 5517.729 & 10 & 18118.37 & $22468^{\circ}{ }_{11 / 2}-40586_{13 / 2}$ & & & & 5422.301 & 2 & 18437.23 & $25745_{\mathrm{s} / 2}-44182_{\mathrm{s} / 2}^{\circ}$ & & & \\
\hline 5517.711 & 2 & 18118.42 & & & & & 5421.661 & 3 & 18439.41 & $18837_{9 / 2}-37276_{11 / 2}^{\circ}$ & & & \\
\hline 5517.115 & 2 & 18120.38 & & & & & 5420.037 & 2 & 18444.93 & $24611_{\mathrm{s} / 2}^{\circ}-43056_{\mathrm{s} / 2}$ & & & \\
\hline 5514.784 & 1 & 18128.04 & $26646_{9 / 2}-44774_{9 / 2}^{\circ}$ & & & & 5419.733 & 1 & 18445.97 & $26701_{7 / 2}-45147_{9 / 2}^{\circ}$ & & & \\
\hline 5513.649 & 1 & 18131.77 & & & & & 5418.438 & 30 & 18450.37 & $22742^{\circ}{ }_{13 / 2}-41193_{13 / 2}$ & $\Delta \mathrm{J}=0$ & 1.19 & 0.16 \\
\hline 5513.566 & 1 & 18132.05 & & & & & 5416.260 & 3 & 18457.79 & $19753_{7 / 2}-38211_{7 / 2}^{\circ}$ & & & \\
\hline 5512.105 & 3 & 18136.85 & & & & & 5414.093 & 4 & 18465.18 & $25717_{7 / 2}-44182_{s / 2}^{\circ}$ & & & \\
\hline 5511.425 & 3 & 18139.09 & $23941_{9 / 2}^{\circ}-42080_{11 / 2}$ & & & & 5413.727 & 100 & 18466.43 & $18693_{15 / 2}-37159^{\circ}{ }_{15 / 2}^{2}$ & $\Delta \mathrm{J}=0$ & 1.20 & 0.38 \\
\hline 5509.890 & 1 & 18144.14 & $22419_{9 / 2}^{\circ}-40563_{9 / 2}$ & & & & 5413.570 & 5 & 18466.97 & & & & \\
\hline 5507.311 & 10 & 18152.64 & & $\Delta \mathrm{J}=0$ & 1.14 & 0.20 & 5413.476 & 10 & 18467.29 & & $\mathrm{~T}$ & 1.13 & 0.06 \\
\hline 5506.495 & 2 & 18155.33 & $22929_{5 / 2}-41085^{\circ}{ }_{7 / 2}$ & & & & 5408.639 & 20 & 18483.80 & $25130_{15 / 2}-43614_{15 / 2}^{\circ}$ & & & \\
\hline 5506.075 & 40 & 18156.71 & $18853_{11 / 2}-37010_{9 / 2}^{\circ}$ & SI & 1.36 & 0.20 & 5407.320 & 1 & 18488.31 & $23941_{9 / 2}^{\circ}-42429_{11 / 2}$ & & & \\
\hline 5505.999 & 1 & 18156.96 & & & & & 5404.640 & 2 & 18497.48 & $21161_{5 / 2}-39658_{s / 2}^{\circ}$ & & & \\
\hline 5505.901 & 10 & 18157.29 & $26357^{\circ}{ }_{13 / 2}-44515_{13 / 2}$ & & & & & & & $23873_{7 / 2}-42370_{7 / 2}^{\circ}$ & & & \\
\hline 5503.466 & 2 & 18165.32 & & & & & 5403.990 & 1 & 18499.70 & $23374_{13 / 2}-41874_{11 / 2}$ & & & \\
\hline 5501.340 & 20 & 18172.34 & & & & & 5403.628 & 2 & 18500.94 & & & & \\
\hline 5501.093 & 60 & 18173.16 & $18837_{9 / 2}-37010_{9 / 2}^{\circ}$ & $\Delta \mathrm{J}=0$ & 1.12 & 0.10 & 5402.716 & 40 & 18504.06 & $17454_{13 / 2}-35958^{\circ}{ }_{13 / 2}$ & $\Delta \mathrm{J}=0$ & 1.14 & 0.15 \\
\hline 5497.391 & 3 & 18185.39 & $23431^{\circ}{ }_{s / 2}-41617_{7 / 2}$ & & & & 5402.231 & 200 & 18505.73 & & $\Delta \mathrm{J}=0$ & 1.22 & 0.73 \\
\hline 5495.616 & 30 & 18191.27 & $19466_{13 / 2}-37657^{\circ}{ }_{11 / 2}$ & SI & 1.95 & 0.77 & 5400.600 & 10 & 18511.31 & $18853_{11 / 2}-37365^{\circ}{ }_{13 / 2}$ & SI & 1.39 & 0.20 \\
\hline 5495.189 & 1 & 18192.68 & $24348_{9 / 2}-42541^{\circ}{ }_{11 / 2}$ & & & & 5391.912 & 50 & 18541.14 & $15271_{15 / 2}-33812^{\circ}{ }_{13 / 2}$ & & & \\
\hline 5494.617 & 50 & 18194.58 & $25520^{\circ}{ }_{11 / 2}-43715_{11 / 2}$ & & & & 5391.500 & 3 & 18542.56 & $23374_{13 / 2}-41917^{\circ}{ }_{13 / 2}$ & & & \\
\hline 5493.950 & 4 & 18196.78 & $24160^{\circ}{ }_{1 / 2}-42357_{3 / 2}$ & & & & 5391.172 & 1 & 18543.69 & & & & \\
\hline 5491.365 & 10 & 18205.35 & $15587_{11 / 2}-33793^{\circ}{ }_{11 / 2}$ & $\mathrm{~T}$ & 1.26 & & 5390.460 & 15 & 18546.14 & $19466_{13 / 2}-38012^{\circ}{ }_{13 / 2}$ & & & \\
\hline 5490.046 & 3 & 18209.72 & & & & & 5389.814 & 5 & 18548.36 & & & & \\
\hline 5489.104 & 3 & 18212.85 & $24348_{9 / 2}-42561_{9 / 2}^{\circ}$ & & & & 5386.736 & 10 & 18558.96 & $18693_{15 / 2}-37252^{\circ}{ }_{17 / 2}$ & SI & 1.46 & 0.245 \\
\hline 5485.645 & 200 & 18224.33 & $15587_{11 / 2}^{/ 2}-33812^{\circ}{ }_{13 / 2}$ & so & 0.56 & 0.58 & 5382.044 & 15 & 18575.14 & $13119_{9 / 2}-31694^{\circ / 2}$ & SI & 1.77 & 0.45 \\
\hline 5483.420 & 3 & 18231.73 & $27377_{13 / 2}-45608^{\circ}{ }_{15 / 2}$ & & & & 5377.280 & 4 & 18591.59 & $22559^{\circ}{ }_{11 / 2}-4115 l_{9 / 2}$ & & & \\
\hline 5483.200 & 2 & 18232.46 & & & & & 5376.834 & 3 & 18593.14 & $25207_{3 / 2}^{\circ}-43800_{3 / 2}$ & & & \\
\hline
\end{tabular}


APPENDIX - Spectral Lines of Neutral Thulium - Continued

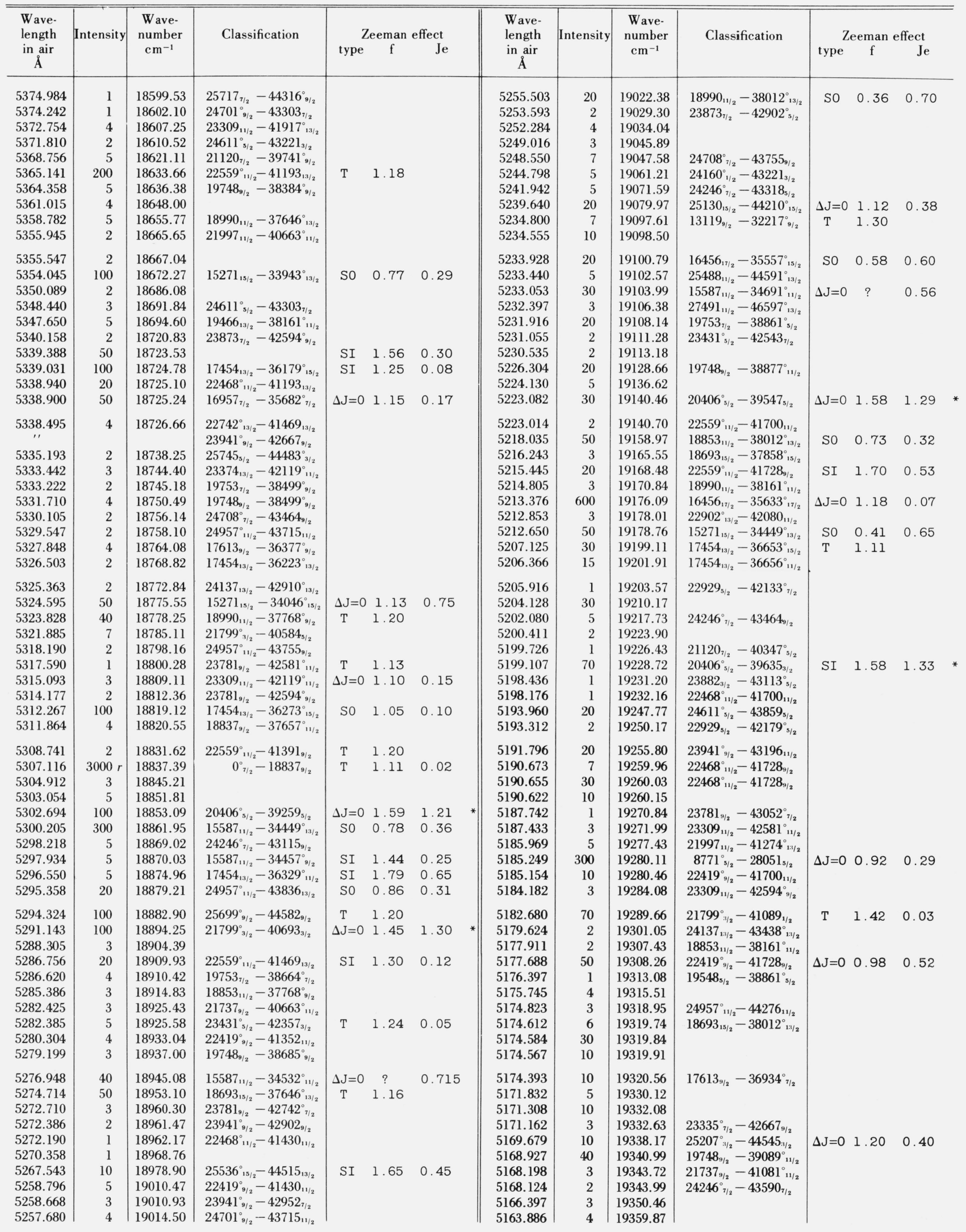


APPENDIX - Spectral Lines of Neutral Thulium-Continued

\begin{tabular}{|c|c|c|c|c|c|c|c|c|c|c|c|c|c|c|}
\hline \multirow{2}{*}{$\begin{array}{c}\text { Wave- } \\
\text { length } \\
\text { in air } \\
\AA\end{array}$} & \multirow{2}{*}{ Intensity } & \multirow{2}{*}{$\begin{array}{c}\text { Wave- } \\
\text { number } \\
\mathrm{cm}^{-1}\end{array}$} & \multirow[t]{2}{*}{ Classification } & \multirow{2}{*}{\multicolumn{4}{|c|}{$\begin{array}{l}\text { Zeeman effect } \\
\text { f } \quad \mathrm{Je}\end{array}$}} & \multirow{2}{*}{$\begin{array}{c}\text { Wave- } \\
\text { length } \\
\text { in air } \\
\AA\end{array}$} & Intensity & $\begin{array}{l}\text { Wave- } \\
\text { number }\end{array}$ & Classification & & Zeeman e & ffect \\
\hline & & & & & & & & & & $\mathrm{cm}^{-1}$ & & type & f & Je \\
\hline 5163.290 & 1 & 19362.10 & $23941_{9 / 2}^{\circ}-43303_{7 / 2}$ & & & & & 5084.950 & 20 & 19660.40 & $22419_{9 / 2}^{\circ}-42080_{11 / 2}$ & & & \\
\hline 5160.543 & 200 & 19372.41 & $8771_{\mathrm{s} / 2}^{\circ}-28143_{3 / 2}$ & SI & 0.85 & 0.65 & * & 5084.624 & 6 & 19661.66 & $18837_{9 / 2}-38499_{9 / 2}^{\circ}$ & & & \\
\hline 5160.053 & 20 & 19374.25 & $18837_{9 / 2}-38211^{\circ}{ }_{7 / 2}$ & & & & & 5084.244 & 4 & 19663.13 & $17613_{9 / 2}-37276_{11 / 2}^{\circ}$ & & & \\
\hline 5156.207 & 2 & 19388.70 & $22791_{7 / 2}-42179_{\mathrm{s} / 2}^{\circ}$ & & & & & 5083.914 & 5 & 19664.40 & & & & \\
\hline 5154.482 & 3 & 19395.19 & & & & & & 5083.402 & 10 & 19666.38 & & & & \\
\hline 5154.034 & 10 & 19396.87 & $21120_{\tau / 2}-40517^{\circ}{ }_{9 / 2}$ & & & & & 5083.197 & 5 & 19667.18 & $17343_{\tau / 2}-37010^{\circ}{ }_{9 / 2}$ & & & \\
\hline 5153.555 & 2 & 19398.68 & & & & & & 5081.729 & 20 & 19672.86 & $24708^{\circ}{ }_{7 / 2}-44380_{9 / 2}$ & SI & 1.40 & 0.25 \\
\hline 5150.405 & 5 & 19410.54 & $19466_{1: 1 / 2}-38877^{\circ}{ }_{11 / 2}$ & & & & & 5081.643 & 3 & 19673.19 & & & & \\
\hline 5149.600 & 10 & 19413.58 & $23941_{9 / 2}^{\circ}-43354_{9 / 2}$ & & & & & 5080.948 & 1 & 19675.88 & $18853_{11 / 2}-38529^{\circ}{ }_{11 / 2}$ & & & \\
\hline 5147.705 & 50 & 19420.72 & $16957_{\tau^{2} / 2}-36377_{9 / 2}^{\circ}$ & SI & 1.33 & 0.12 & & 5080.577 & 20 & 19677.32 & $8771_{s / 2}^{\circ}-28448_{s / 2}$ & $\Delta \mathrm{J}=0$ & 0.94 & 0.33 \\
\hline 5147.172 & 4 & 19422.73 & & & & & & 5079.925 & 1 & 19679.84 & $24701_{9 / 2}^{\circ}-44380_{9 / 2}$ & & & \\
\hline 5145.367 & 5 & 19429.55 & $23335_{7 / 2}^{\circ}-42764_{9 / 2}$ & & & & & 5079.675 & 6 & 19680.81 & & & & \\
\hline 5142.886 & 300 & 19438.92 & $19748_{9 / 2}-39187^{\circ}{ }_{7 / 2}^{\circ}$ & so & 1.09 & 0.18 & & 5078.180 & 40 & 19686.61 & $22742^{\circ}{ }_{13 / 2}-42429_{11 / 2}$ & $\mathrm{~T}$ & 1.20 & \\
\hline 5142.545 & 100 & 19440.21 & $20406^{\circ}{ }^{\circ}-39847_{7 / 2}$ & so & 0.54 & 0.73 & & 5077.312 & 2 & 19689.97 & $21997_{11 / 2}-41687^{\circ}{ }_{11 / 2}$ & & & \\
\hline 5141.405 & 4 & 19444.52 & $23431_{\mathrm{s} / 2}^{\circ}-42876_{\mathrm{s} / 2}$ & & & & & 5077.183 & 300 & 19690.47 & $19753_{\tau / 2}-39444_{9 / 2}^{\circ}$ & so & 0.90 & 0.21 \\
\hline 5140.392 & 4 & 19448.35 & $23335^{\circ}{ }_{7 / 2}-42783_{7 / 2}$ & & & & & 5076.875 & 20 & 19691.67 & & & & \\
\hline 5137.310 & 3 & 19460.02 & $24957^{\circ}{ }_{11 / 2}-44417_{11 / 2}$ & & & & & 5076.705 & 2 & 19692.33 & $18837_{9 / 2}-38529^{\circ}{ }_{11 / 2}$ & & & \\
\hline 5137.102 & 2 & 19460.81 & $23309_{11 / 2}-42770_{9 / 2}^{\circ}$ & & & & & 5076.469 & 10 & 19693.24 & & & & \\
\hline 5135.682 & 10 & 19466.19 & $19753_{\tau_{/ 2}}-39220_{9 / 2}^{\circ}$ & & & & & 5076.360 & 200 & 19693.67 & $21799_{3 / 2}^{\circ}-41493_{\mathrm{s} / 2}$ & so & 1.05 & 0.24 \\
\hline 5135.503 & 15 & 19466.87 & $15587_{11 / 2}-35054_{9 / 2}^{\circ}$ & so & 1.11 & 0.14 & & 5075.970 & 2 & 19695.18 & $18990_{11 / 2}^{/ 2}-38685_{9 / 2}^{\circ}$ & & & \\
\hline 5134.282 & 20 & 19471.50 & $19748_{9 / 2}-39220_{9 / 2}^{\circ}$ & & & & & 5075.820 & 60 & 19695.76 & $19748_{9 / 2}-39444_{9 / 2}^{\circ}$ & $\Delta \mathrm{J}=0$ & 1.185 & 0.66 \\
\hline 5131.564 & 4 & 19481.81 & $23574_{: 3 / 2}^{\circ}-43056_{s / 2}$ & & & & & 5073.513 & 100 & 19704.72 & $17454_{13 / 2}-37159^{\circ}{ }_{15 / 2}$ & SI & 1.80 & 0.58 \\
\hline 5129.526 & 4 & 19489.55 & & & & & & 5072.416 & 300 & 19708.98 & $19753_{\tau_{12}}-39462^{\circ}{ }_{7 / 2}^{\circ}$ & $\Delta \mathrm{J}=0$ & 1.15 & 0.20 \\
\hline 5129.210 & 3 & 19490.75 & $19753_{7 / 2}-39244_{9 / 2}^{\circ}$ & & & & & 5071.833 & 1 & 19711.24 & & & & \\
\hline 5129.127 & 15 & 19491.06 & & so & 0.83 & 0.23 & & 5071.717 & 3 & 19711.69 & $24611_{s / 2}^{\circ}-44323_{7 / 2}$ & & & \\
\hline 5127.819 & 20 & 19496.04 & $19748_{9 / 2}-39244_{9 / 2}^{\circ}$ & & & & & 5071.200 & 20 & 19713.70 & $22742^{\circ}{ }_{13 / 2}-42456_{13 / 2}$ & $\Delta \mathrm{J}=0$ & 1.22 & 0.10 \\
\hline 5127.215 & 2 & 19498.33 & $24708^{\circ}{ }_{7 / 2}^{\circ}-44206_{\tau / 2}$ & & & & & 5071.057 & 30 & 19714.26 & $19748_{9 / 2}-39462_{7 / 2}^{\circ}$ & SI & 1.92 & 0.63 \\
\hline 5125.593 & 2 & 19504.50 & & & & & & 5070.494 & 20 & 19716.45 & $24701_{9 / 2}^{\circ}-44417_{11 / 2}^{1 / 2}$ & $\mathrm{~T}$ & 1.15 & \\
\hline 5124.516 & 7 & 19508.60 & $18990_{11 / 2}-38499_{9 / 2}^{\circ}$ & & & & & 5070.432 & 10 & 19716.69 & $19753_{7 / 2}^{/ 2}-39470^{\circ}{ }_{s / 2}$ & & & \\
\hline 5123.370 & 30 & 19512.97 & $19548_{s / 2}-39061_{: 3 / 2}^{\circ}$ & & & & & 5069.817 & 3 & 19719.08 & & & & \\
\hline 5121.353 & 3 & 19520.65 & $22559^{\circ}{ }_{11 / 2}-42080_{11 / 2}$ & & & & & 5069.300 & 2 & 19721.09 & $23335^{\circ}{ }_{7 / 2}-43056_{3 / 2}$ & & & \\
\hline 5120.671 & 150 & 19523.25 & $19753_{\tau_{/ 2}}-39277_{\mathrm{s} / 2}^{\circ}$ & $\mathrm{T}$ & 1.18 & & & 5068.890 & 50 & 19722.69 & $16456_{17 / 2}-36179^{\circ}{ }_{15 / 2}$ & SI & 1.29 & 0.14 \\
\hline 5118.610 & 4 & 19531.11 & $18853_{11 / 2}-38384_{9 / 2}^{\circ}$ & & & & & 5067.918 & 10 & 19726.47 & $19753_{7 / 2}-39480^{\circ}{ }_{7 / 2}$ & & & \\
\hline 5115.953 & $5 h$ & 19541.26 & $23335_{7 / 2}^{\circ}-42876_{\mathrm{s} / 2}$ & & & & & 5067.825 & 30 & 19726.83 & & & & \\
\hline 5113.970 & $500 r$ & 19548.83 & $0_{7 / 2}^{\circ}-19548_{s / 2}$ & SI & 1.53 & 0.39 & & 5067.463 & 30 & 19728.24 & $19548_{s / 2}-39277_{s / 2}^{\circ}$ & & & \\
\hline 5113.890 & 10 & 19549.14 & & & & & & 5066.670 & 800 & 19731.33 & $19748_{1 / 2}-39479_{11 / 2}^{\circ}$ & so & 0.86 & 0.36 \\
\hline 5112.482 & 20 & 19554.52 & & & & & & 5065.876 & 100 & 19734.42 & $19753_{\tau_{7 / 2}}-39488^{\circ}{ }_{9 / 2}^{\circ}$ & SI & 1.40 & 0.35 \\
\hline 5112.065 & 2 & 19556.12 & $23873_{7 / 2}-43429_{9 / 2}^{\circ}$ & & & & & 5065.812 & 30 & 19734.67 & $18853_{11 / 2}^{1 / 2}-38588^{\circ}{ }_{1: 1 / 2}^{1 / 2}$ & & & \\
\hline 5111.776 & 7 & 19557.22 & $24708^{\circ}{ }_{7 / 2}^{2}-44265_{7 / 2}$ & & & & & & & & $24246^{\circ}{ }_{\tau / 2}-43981_{\tau / 2}$ & & & \\
\hline 5111.691 & 30 & 19557.55 & $24957^{\circ}{ }_{11 / 2}-44515_{13 / 2}$ & so & 0.56 & 0.56 & & 5064.523 & 10 & 19739.69 & $19748_{9 / 2}-39488^{\circ}{ }_{9 / 2}^{\circ}$ & & & \\
\hline 5109.476 & 1 & 19566.03 & & & & & & 5062.250 & 200 & 19748.56 & $0_{7 / 2}^{\circ}-19748_{1 / 2}$ & SI & 1.143 & 1.29 \\
\hline 5109.103 & 2 & 19567.45 & $23335_{7 / 2}^{\circ}-42902_{9 / 2}$ & & & & & 5060.895 & $2000 r$ & 19753.84 & $0_{7 / 2}^{\circ}-19753_{7 / 2}^{\prime}$ & $\Delta \mathrm{J}=0$ & 1.15 & 0.13 \\
\hline 5108.426 & 1 & 19570.05 & $25520^{\circ}{ }_{11 / 2}-45091_{9 / 2}$ & & & & & 5058.208 & 2 & 19764.34 & $24418_{s / 2}^{1 / 2}-44182^{\circ / 2}$ & & & \\
\hline 5107.533 & 400 & 19573.47 & $19748_{9 / 2}-39322_{9 / 2}^{\circ}$ & $\Delta \mathrm{J}=0$ & 1.22 & 0.64 & & 5057.559 & 5 & 19766.87 & $17752_{s / 2}-37519^{\circ}{ }_{7 / 2}$ & & & \\
\hline 5106.760 & 2 & 19576.43 & $22791_{7 / 2}-42367_{\mathrm{s} / 2}^{\circ}$ & & & & & 5056.182 & 60 & 19772.26 & & $\mathrm{~T}$ & 1.27 & 0.07 \\
\hline 5106.174 & 2 & 19578.68 & $21997_{11 / 2}-41576_{9 / 2}^{\circ}$ & & & & & 5055.227 & 5 & 19775.99 & $15587_{11 / 2}-35363_{9 / 2}^{\circ}$ & & & \\
\hline 5105.526 & 2 & 19581.16 & & & & & & 5054.909 & 1 & 19777.24 & $21161_{s / 2}-40938_{s / 2}^{\circ}$ & & & \\
\hline 5104.237 & 2 & 19586.11 & $23574_{3 / 2}^{\circ}-43160_{3 / 2}$ & & & & & 5054.619 & 3 & 19778.37 & & & & \\
\hline 5103.387 & 20 & 19589.37 & $20406_{\mathrm{s} / 2}^{\circ}-39996_{\tau / 2}$ & & & & & 5054.119 & 10 & 19780.33 & $23335_{7 / 2}^{\circ}-43115_{9 / 2}$ & & & \\
\hline 5103.006 & 1 & 19590.83 & $17343_{7 / 2}-36934^{\circ}{ }_{7 / 2}^{\prime}$ & & & & & 5053.043 & 150 & 19784.54 & $8771_{s / 2}^{s}-28555_{7 / 2}$ & SI & 0.85 & 1.15 \\
\hline 5101.896 & 10 & 19595.10 & $24611_{s / 2}^{\circ}-44206_{7 / 2}$ & SI & 1.35 & 0.29 & & 5051.648 & 20 & 19790.00 & $23431_{s / 2}^{\circ}-43221_{: 3 / 2}$ & SI & 1.29 & 0.06 \\
\hline 5101.821 & 2 & 19595.38 & & & & & & 5050.935 & 2 & 19792.80 & $18693_{15 / 2}-38485^{\circ}{ }_{15 / 2}$ & & & \\
\hline 5101.129 & 40 & 19598.04 & $18990_{11 / 2}-38588^{\circ}{ }_{13 / 2}$ & $\mathrm{~T}$ & 1.22 & & & 5049.798 & 2 & 19797.25 & & & & \\
\hline 5097.469 & 2 & 19612.11 & $22468_{11 / 2}^{\circ}-42080_{11 / 2}$ & & & & & 5048.493 & 2 & 19802.37 & $22742^{\circ}{ }_{1: / 2}-42545_{1: 1 / 2}$ & & & \\
\hline 5096.222 & 2 & 19616.91 & $23335^{\circ}{ }_{7 / 2}-42952_{\tau_{7 / 2}}$ & & & & & 5046.254 & 1 & 19811.16 & $23431_{\mathrm{s} / 2}^{\circ}-43243_{\mathrm{s} / 2}$ & & & \\
\hline 5095.530 & 2 & 19619.58 & $19132_{3 / 2}-38751^{\circ}{ }_{s / 2}$ & & & & & 5046.177 & 2 & 19811.46 & & & & \\
\hline 5094.641 & 2 & 19623.00 & & & & & & 5045.963 & 10 & 19812.30 & $22929_{s / 2}-42742_{\tau / 2}^{\circ}$ & & & \\
\hline 5094.277 & 30 & 19624.40 & $23431_{s / 2}^{\circ}-43056_{s / 2}$ & & & & & 5045.408 & 250 & 19814.48 & $19748_{9 / 2}-39563^{\circ}{ }_{11 / 2}$ & so & 0.23 & 0.86 \\
\hline 5092.277 & 2 & 19632.11 & $24611_{s / 2}^{\circ}-44243_{s / 2}$ & & & & & 5044.758 & 2 & 19817.03 & $16456_{17 / 2}-36273^{\circ}{ }_{13 / 2}$ & & & \\
\hline 5092.009 & 4 & 19633.14 & $19753_{7 / 2}-39386_{s / 2}^{\circ}$ & & & & & 5043.505 & 100 & 19821.95 & $17454_{1: 1 / 2}-37276^{\circ}{ }_{11 / 2}^{1 / 2}$ & so & 0.61 & 0.54 \\
\hline 5091.391 & 2 & 19635.53 & $16742_{7 / 2}-36377_{9 / 2}^{\circ}$ & & & & & 5043.238 & 5 & 19823.00 & & & & \\
\hline 5090.162 & 7 & 19640.27 & $24160^{\circ}{ }_{1 / 2}-43800_{3 / 2}$ & & & & & 5041.213 & 20 & 19830.97 & $22902^{\circ}{ }_{1: 1 / 2}-42733_{15 / 2}$ & & & \\
\hline 5089.437 & 2 & 19643.06 & $22902^{\circ}{ }_{13 / 2}-42545_{13 / 2}$ & & & & & 5040.738 & 20 & 19832.83 & & & & \\
\hline & & & $21120_{7 / 2}-40763^{\circ}{ }_{7 / 2}$ & & & & & 5039.393 & 20 & 19838.13 & $19548_{s / 2}-39386_{s / 2}^{\circ}$ & $\mathrm{T}$ & 0.99 & \\
\hline 5088.881 & 40 & 19645.21 & $18853_{11 / 2}^{/ 2}-38499_{9 / 2}^{\circ}$ & & & & & 5037.057 & 4 & 19847.33 & $23941^{\circ} 1 / 2-43788_{11 / 2}^{/ 2}$ & & & \\
\hline & & & $18837_{9 / 2}-38482^{\circ}{ }_{7 / 2}$ & & & & & 5036.129 & 1 & 19850.99 & $21161_{s / 2}-41012^{\circ}{ }_{\tau / 2}$ & & & \\
\hline 5088.302 & 15 & 19647.45 & $23574_{3 / 2}^{\circ}-43221_{3 / 2}$ & & & & & 5035.618 & 20 & 19853.00 & $22742^{\circ}{ }_{1: 1 / 2}-42595_{11 / 2}$ & $\mathrm{~T}$ & 1.21 & \\
\hline 5087.323 & 10 & 19651.23 & & & & & & 5032.620 & 6 & 19864.83 & $19548_{s / 2}-39413^{\circ}{ }_{7 / 2}$ & & & \\
\hline 5086.607 & 7 & 19653.99 & $24611_{s / 2}^{\circ}-44265_{\tau_{7 / 2}}$ & & & & & 5032.183 & 2 & 19866.55 & & & & \\
\hline 5085.086 & 200 & 19659.87 & $20406_{s / 2}^{\circ}-40066_{s / 2}^{\circ}$ & $\Delta \mathrm{J}=0$ & 1.58 & 1.36 & $* \|$ & 5031.341 & 3 & 19869.88 & $22559^{\circ}{ }_{11 / 2}-42429_{11 / 2}$ & & & \\
\hline
\end{tabular}


APPENDIX - Spectral Lines of Neutral Thulium-Continued

\begin{tabular}{|c|c|c|c|c|c|c|c|c|c|c|c|c|c|c|}
\hline \multirow{2}{*}{$\begin{array}{c}\text { Wave- } \\
\text { length } \\
\text { in air } \\
\AA\end{array}$} & \multirow[t]{2}{*}{ Intensity } & \multirow{2}{*}{$\begin{array}{l}\text { Wave- } \\
\text { number } \\
\mathrm{cm}^{-1}\end{array}$} & \multirow[t]{2}{*}{ Classification } & \multirow{2}{*}{\multicolumn{4}{|c|}{$\begin{array}{l}\text { Zeeman effect } \\
\mathrm{f} \quad \mathrm{Je}\end{array}$}} & \multirow{2}{*}{$\begin{array}{c}\text { Wave- } \\
\text { length } \\
\text { in air } \\
\AA\end{array}$} & Intensity & $\begin{array}{l}\text { Wave- } \\
\text { number }\end{array}$ & Classification & & eeman & \\
\hline & & & & & & & & & & $\mathrm{cm}^{-1}$ & & type & f & $\mathrm{Je}$ \\
\hline 5031.042 & 9 & 19871.06 & $19548_{5 / 2}-39419^{\circ}{ }_{3 / 2}$ & & & & & 4962.673 & 3 & 20144.81 & $19132_{3 / 2}-39277_{5 / 2}^{\circ}$ & & & \\
\hline 5030.260 & 200 & 19874.15 & $19753_{\tau / 2}-39628^{\circ}{ }_{\tau / 2}$ & $\Delta \mathrm{J}=0$ & 1.10 & 0.51 & & 4962.327 & $5 h$ & 20146.21 & $23335^{\circ}{ }_{7 / 2}-43481_{5 / 2}$ & & & \\
\hline 5029.262 & 1 & 19878.09 & $17343_{7 / 2}-37221^{\circ}{ }_{7 / 2}^{\circ}$ & & & & & 4961.564 & 10 & 20149.31 & & & & \\
\hline 5028.608 & 1 & 19880.68 & & & & & & 4960.163 & 10 & 20155.00 & $17613_{9_{/ 2}}-37768_{9 / 2}^{\circ}$ & & & \\
\hline 5028.228 & 4 & 19882.18 & $24418_{5 / 2}-44300_{5 / 2}^{\circ}$ & & & & & 4959.855 & 4 & 20156.26 & $22419^{\circ / 2}{ }_{9 / 2}-42576_{9 / 2}^{9 / 2}$ & & & \\
\hline 5027.060 & 10 & 19886.80 & $18990_{11 / 2}-38877^{\circ}{ }_{11 / 2}$ & $\Delta \mathrm{J}=0$ & 1.15 & 0.84 & & 4959.283 & 2 & 20158.58 & $23431^{\circ / 2}-43590_{7 / 2}$ & & & \\
\hline 5025.860 & 2 & 19891.55 & $21120_{7 / 2}-41012^{\circ}{ }_{7 / 2}^{\circ}$ & & & & & 4957.175 & 1000 & 20167.15 & $18990_{11 / 2}-39157^{\circ}{ }_{13 / 2}$ & SI & 1.38 & 0.14 \\
\hline 5024.890 & 50 & 19895.38 & $18693_{15 / 2}-38588^{\circ}{ }_{13 / 2}$ & so & 0.89 & 0.26 & & 4956.474 & 1 & 20170.00 & $24137_{13 / 2}-44307^{\circ}{ }_{13 / 2}$ & & & \\
\hline 5024.429 & 1 & 19897.21 & & & & & & 4954.978 & 2 & 20176.09 & $17343_{\tau / 2}-37519^{\circ}{ }_{\tau / 2}$ & & & \\
\hline 5023.681 & 4 & 19900.17 & & & & & & 4954.585 & 5 & 20177.69 & $20406_{5 / 2}^{\circ / 2}-40584_{5 / 2}^{1 / 2}$ & & & \\
\hline 5023.597 & 5 & 19900.51 & $25488_{11 / 2}-45389_{13 / 2}^{\circ}$ & & & & & 4954.511 & 3 & 20178.00 & & & & \\
\hline 5022.457 & 4 & 19905.02 & $19753_{7 / 2}-39658^{\circ}{ }_{s / 2}$ & & & & & 4953.834 & 2 & 20180.75 & & & & \\
\hline 5022.198 & 3 & 19906.05 & $22419_{9 / 2}^{\circ}-42325_{\tau_{/ 2}}^{\prime}$ & & & & & 4952.266 & 2 & 20187.14 & & & & \\
\hline 5021.123 & 100 & 19910.31 & $17454_{1: 3 / 2}-37365_{13 / 2}^{\circ}$ & $\Delta \mathrm{J}=0$ & 1.18 & 0.35 & & 4951.704 & 1 & 20189.43 & & & & \\
\hline 5019.156 & 2 & 19918.11 & $24611_{5 / 2}^{\circ}-44529_{\tau^{2} / 2}$ & & & & & 4951.229 & 1 & 20191.37 & $17454_{13 / 2}-37646^{\circ}{ }_{13 / 2}$ & & & \\
\hline 5018.737 & 20 & 19919.78 & $21997_{11 / 2}-41917^{\circ}{ }_{13 / 2}$ & & & & & 4951.080 & 2 & 20191.98 & $16742_{\tau / 2}-36934^{\circ}{ }_{7 / 2}$ & & & \\
\hline " & & & $23574_{: 3 / 2}^{0}-43494_{s / 2}$ & & & & & 4949.845 & 5 & 20197.02 & $16456_{17 / 2}-36653^{\circ}{ }_{15 / 2}$ & & & \\
\hline 5018.442 & 5 & 19920.95 & & & & & & 4949.188 & 1 & 20199.70 & $22468^{\circ}{ }_{11 / 2}-42667_{9 / 2}$ & & & \\
\hline 5017.303 & 40 & 19925.47 & $18990_{11 / 2}-38915_{1: 1 / 2}^{\circ}$ & $\mathrm{T}$ & 1.25 & 0.04 & & 4948.670 & 7 & 20201.81 & & $\mathrm{~T}$ & 0.98 & \\
\hline 5016.272 & 10 & 19929.56 & $19132_{: 3 / 2}-39061_{: 3 / 2}^{\circ}$ & & & & & 4948.353 & 100 & 20203.11 & $17454_{13 / 2}-37657^{\circ}{ }_{11 / 2}$ & SI & 1.84 & 0.69 \\
\hline 5014.277 & 50 & 19937.49 & & $\mathrm{~T}$ & 0.70 & & & 4947.852 & 5 & 20205.15 & $22559^{\circ}{ }_{11 / 2}-42764_{9 / 2}$ & & & \\
\hline 5013.281 & 3 & 19941.45 & & & & & & 4947.497 & 30 & 20206.60 & & $\mathrm{~T}$ & 1.10 & \\
\hline 5010.718 & 20 & 19951.65 & $21799^{\circ}: 3 / 2-41751_{s / 2}$ & so & 1.45 & 1.08 & * & 4946.358 & 3 & 20211.25 & $24708^{\circ}{ }_{7 / 2}-44919_{9 / 2}$ & & & \\
\hline 5010.245 & 30 & 19953.54 & $20406_{s / 2}^{\circ}-40360_{7 / 2}$ & & & & & 4945.600 & 1 & 20214.35 & $22742^{\circ}{ }_{13 / 2}-42957_{11 / 2}$ & & & \\
\hline & & & $21120_{7 / 2}-41074_{s_{s / 2}}^{\circ}$ & & & & & 4945.299 & 2 & 20215.58 & & & & \\
\hline 5009.690 & 10 & 19955.75 & & & & & & 4944.833 & 2 & 20217.49 & & & & \\
\hline 5008.497 & 1 & 19960.50 & $19748_{9 / 2}-39709^{\circ}{ }_{11 / 2}$ & & & & & 4944.652 & 10 & 20218.23 & $24701_{9 / 2}^{\circ}-44919_{9 / 2}$ & $\Delta \mathrm{J}=0$ & $?$ & 0.19 \\
\hline 5008.163 & 1 & 19961.83 & $24957^{\circ}{ }_{11 / 2}-44919_{9 / 2}$ & & & & & 4943.300 & 2 & 20223.76 & & & & \\
\hline 5007.092 & 20 & 19966.10 & $22902^{\circ}{ }_{1: 1 / 2}-42868_{11 / 2}$ & & & & & 4942.640 & 30 & 20226.46 & $23574_{3 / 2}^{\circ}-43800_{3 / 2}$ & $\Delta \mathrm{J}=0$ & 0.83 & 1.20 \\
\hline 5006.736 & 10 & 19967.52 & $19466_{1: 3 / 2}-39434_{13 / 2}^{\circ}$ & & & & & 4942.199 & 1 & 20228.26 & & & & \\
\hline 5006.703 & 2 & 19967.65 & $22902^{\circ}{ }_{13 / 2}-42869_{1: 3 / 2}$ & & & & & 4941.865 & 3 & 20229.63 & $18990_{11 / 2}-39220_{9 / 2}^{\circ}$ & & & \\
\hline $\begin{array}{c}5006.607 \\
, 1\end{array}$ & 10 & 19968.04 & $\begin{array}{l}24348_{9 / 2}-44316_{9 / 2}^{\circ} \\
23335^{\circ}{ }_{7 / 2}-43303_{7 / 2}\end{array}$ & & & & & $\begin{array}{l}4940.383 \\
4940.187\end{array}$ & $\begin{array}{r}20 \\
3\end{array}$ & $\begin{array}{l}20235.70 \\
20236.50\end{array}$ & $\begin{array}{l}18853_{11 / 2}^{1 / 2}-39089^{\circ}{ }_{11 / 2} \\
23335^{\circ}-43571_{5 / 2}\end{array}$ & $\Delta \mathrm{J}=0$ & 1.13 & 0.25 \\
\hline 5003.814 & 2 & 19979.18 & $25488_{11 / 2}^{7 / 2}-45467_{9 / 2}^{\circ}$ & & & & & 4939.440 & 3 & 20239.56 & $23374_{13 / 2}-43614^{\circ}{ }_{15 / 2}$ & & & \\
\hline 5003.708 & 3 & 19979.61 & $22791_{7 / 2}-42770_{9 / 2}^{\circ}$ & & & & & 4938.753 & 1 & 20242.38 & $19466_{13 / 2}-39709^{\circ}{ }_{11 / 2}$ & & & \\
\hline 5003.145 & 15 & 19981.85 & $24708^{\circ}{ }_{7 / 2}-44689_{9 / 2}$ & & & & & 4938.698 & 5 & 20242.60 & $20406_{5 / 2}^{\circ}-40649_{7 / 2}$ & & & \\
\hline 5002.197 & 10 & 19985.64 & $22559^{\circ}{ }_{11 / 2}-42545_{13 / 2}$ & & & & & 4937.385 & 20 & 20247.98 & $22419_{9 / 2}^{\circ}-42667_{9 / 2}^{\circ}$ & & & \\
\hline 5001.587 & 200 & 19988.08 & $19753_{7 / 2}-39741_{9 / 2}^{\circ}$ & so & 0.79 & 0.30 & & 4935.880 & 20 & 20254.16 & $18990_{11 / 2}-39244_{9 / 2}^{\circ}$ & & & \\
\hline 5001.504 & 30 & 19988.41 & $22468^{\circ}{ }_{11 / 2}-42456_{13 / 2}$ & & & & & 4935.747 & 3 & 20254.70 & $19132_{3 / 2}-39386_{5 / 2}^{\circ}$ & & & \\
\hline 5001.025 & 200 & 19990.32 & $22742^{\circ}{ }_{13 / 2}-42733_{15 / 2}$ & $\mathrm{~T}$ & 1.12 & 0.07 & & 4934.798 & 2 & 20258.60 & $25130_{15 / 2}-45389^{\circ}{ }_{13 / 2}$ & & & \\
\hline 5000.270 & 100 & 19993.34 & $19548_{5 / 2}-39542^{\circ}{ }_{7 / 2}$ & SI & 1.18 & 0.12 & & 4933.365 & 5 & 20264.48 & $16957_{\tau / 2}-37221^{\circ}{ }_{\tau / 2}$ & & & \\
\hline 4999.643 & 3 & 19995.85 & & & & & & 4933.162 & 1 & 20265.32 & $23941_{9 / 2}^{\circ / 2}-44206_{7 / 2}$ & & & \\
\hline 4996.201 & 1 & 20009.63 & $22419_{9 / 2}^{\circ}-42429_{11 / 2}$ & & & & & 4932.552 & 1 & 20267.82 & $27377_{13 / 2}-4.7644^{\circ}{ }_{13 / 2}$ & & & \\
\hline 4994.480 & 2 & 20016.52 & $22559^{\circ}{ }_{11 / 2}-42576_{9 / 2}$ & & & & & 4932.390 & 2 & 20268.49 & & & & \\
\hline 4992.780 & 20 & 20023.34 & & & & & & 4929.729 & 10 & 20279.43 & & & & \\
\hline 4988.669 & 6 & 20039.84 & $18837_{9 / 2}-38877^{\circ}{ }_{11 / 2}$ & & & & & 4929.228 & 2 & 20281.49 & $24137_{13 / 2}-44418^{\circ}{ }_{13 / 2}$ & & & \\
\hline 4987.139 & 2 & 20045.98 & & & & & & 4928.457 & 50 & 20284.66 & $23574^{\circ}{ }_{3 / 2}-43859_{3 / 2}$ & SI & 1.55 & 0.42 \\
\hline 4986.256 & 1 & 20049.53 & & & & & & 4927.945 & 3 & 20286.77 & $20406^{\circ}{ }_{s / 2}-40693_{3 / 2}$ & & & \\
\hline 4985.627 & 1 & 20052.06 & & & & & & 4927.732 & 200 & 20287.65 & $19132_{3 / 2}-39419^{\circ}{ }_{3 / 2}$ & $\Delta \mathrm{J}=0$ & 0.89 & 0.09 \\
\hline 4985.260 & 5 & 20053.54 & $16957_{\tau / 2}-37010_{9 / 2}^{\circ}$ & & & & & 4925.555 & 1 & 20296.61 & $22468^{\circ}{ }_{11 / 2}-42764_{9 / 2}$ & & & \\
\hline 4985.130 & 200 & 20054.06 & $19548_{5 / 2}-39602_{3 / 2}^{\circ}$ & so & 0.985 & 1.31 & * & 4925.274 & 1 & 20297.77 & $23941_{9 / 2}^{\circ}-44238_{9 / 2}$ & & & \\
\hline 4984.893 & $5 h$ & 20055.02 & $22902^{\circ}{ }_{13 / 2}-42957_{11 / 2}$ & & & & & 4925.060 & 2 & 20298.65 & $22419_{9 / 2}^{\circ}-42718_{\theta_{2}}^{\circ}$ & & & \\
\hline 4983.185 & 1 & 20061.89 & & & & & & 4923.826 & 600 & 20303.74 & $18853_{11 / 2}-39157^{\circ}{ }_{13 / 2}$ & SI & 1.67 & 0.42 \\
\hline 4983.136 & 2 & 20062.09 & $18853_{11 / 2}-38915^{\circ}{ }_{13 / 2}$ & & & & & 4922.524 & 2 & 20309.11 & $23873_{7 / 2}-44182^{\circ}{ }_{5 / 2}^{\circ / 2}$ & & & \\
\hline 4982.328 & 5 & 20065.34 & $24418_{5 / 2}-44483_{3 / 2}^{\circ}$ & & & & & 4922.260 & 2 & 20310.20 & $22559^{\circ}{ }_{11 / 2}-42869_{13 / 2}$ & & & \\
\hline 4979.540 & 3 & 20076.58 & $24246^{\circ}{ }_{7 / 2}-44323_{7 / 2}$ & & & & & 4921.620 & 1 & 20312.84 & $19748_{9 / 2}-40061^{\circ}{ }_{7 / 2}$ & & & \\
\hline 4978.900 & 400 & 20079.16 & $19548_{5 / 2}-39628^{\circ}{ }_{7 / 2}$ & SI & 1.15 & 0.10 & & 4920.876 & 1 & 20315.91 & & & & \\
\hline 4975.036 & 5 & 20094.75 & & & & & & 4917.085 & 20 & 20331.58 & $18990_{11 / 2}-39322_{9 / 2}^{\circ}$ & SI & 1.50 & 0.23 \\
\hline 4973.953 & 30 & 20099.13 & $18990_{11 / 2}-39089^{\circ}{ }_{11 / 2}$ & $\Delta \mathrm{J}=0$ & $?$ & 0.55 & & 4916.180 & 1 & 20335.32 & $23941_{9 / 2}^{\circ}-44276_{11 / 2}$ & & & \\
\hline 4973.282 & 2 & 20101.84 & & & & & & 4915.961 & 3 & 20336.23 & $24246^{\circ}{ }_{7 / 2}-44582_{9 / 2}$ & & & \\
\hline 4971.694 & 10 & 20108.26 & $22559^{\circ}{ }_{11 / 2}-42667_{9 / 2}$ & & & & & 4915.475 & 1 & 20338.24 & $19132_{3 / 2}-39470_{5 / 2}^{\circ}$ & & & \\
\hline 4971.258 & 200 & 20110.02 & $19548_{5 / 2}-39658_{s / 2}^{\circ}$ & $\Delta \mathrm{J}=0$ & 0.97 & 0.08 & & 4913.296 & 2 & 20347.26 & & & & \\
\hline 4967.437 & 2 & 20125.49 & $22742^{\circ}{ }_{13 / 2}-42868_{11 / 2}$ & & & & & 4909.736 & 200 & 20362.01 & $15271_{15 / 2}-35633^{\circ}{ }_{17 / 2}$ & SI & 1.88 & 0.70 \\
\hline 4967.063 & 1 & 20127.01 & $22742^{\circ}{ }_{13 / 2}-42869_{13 / 2}$ & & & & & 4908.727 & 5 & 20366.19 & $18853_{11 / 2}-39220_{9 / 2}^{\circ}$ & & & \\
\hline 4966.880 & 10 & 20127.75 & $22468^{\circ}{ }_{11 / 2}-42595_{11 / 2}$ & & & & & 4907.550 & 30 & 20371.08 & $15587_{11 / 2}-35958^{\circ}{ }_{13 / 2}$ & so & 0.39 & 0.74 \\
\hline 4965.216 & 2 & 20134.49 & $24246^{\circ}{ }_{7 / 2}-44380_{9 / 2}$ & & & & & 4907.269 & 100 & 20372.25 & $18990_{11 / 2}-39362^{\circ}{ }_{11 / 2}$ & $\Delta \mathrm{J}=0$ & 1.18 & 0.44 \\
\hline 4964.672 & 7 & 20136.70 & $21737_{9 / 2}-41874^{\circ}{ }_{11 / 2}$ & & & & & 4904.690 & 2 & 20382.96 & $24708^{\circ}{ }_{7 / 2}-45091_{\theta / 2}$ & & & \\
\hline 4964.370 & 2 & 20137.92 & $23309_{11 / 2}-43447^{\circ}{ }_{11 / 2}$ & & & & & 4902.815 & 20 & 20390.75 & $18853_{11 / 2}-39244_{9 / 2}^{\circ}$ & & & \\
\hline 4963.052 & 2 & 20143.27 & & & & & & " & & & $23873_{7 / 2}-44263^{\circ}{ }_{7 / 2}$ & & & \\
\hline 4962.943 & 2 & 20143.71 & & & & & & 4901.854 & 2 & 20394.75 & & & & \\
\hline
\end{tabular}


APPENDIX - Spectral Lines of Neutral Thulium - Continued

\begin{tabular}{|c|c|c|c|c|c|c|c|c|c|c|c|c|c|}
\hline \multirow{2}{*}{$\begin{array}{c}\text { Wave- } \\
\text { length } \\
\text { in air } \\
\AA\end{array}$} & \multirow[t]{2}{*}{ Intensity } & \multirow{2}{*}{$\begin{array}{c}\text { Wave- } \\
\text { number } \\
\mathrm{cm}^{-1}\end{array}$} & \multirow[t]{2}{*}{ Classification } & \multirow{2}{*}{\multicolumn{3}{|c|}{$\begin{array}{l}\text { Zeeman effect } \\
\text { e } \quad \mathrm{J} \quad \mathrm{Je}\end{array}$}} & \multirow{2}{*}{$\begin{array}{c}\text { Wave- } \\
\text { length } \\
\text { in air } \\
\AA\end{array}$} & Intensity & $\begin{array}{l}\text { Wave- } \\
\text { number }\end{array}$ & Classification & & eeman $e$ & ffect \\
\hline & & & & & & & & & $\mathrm{cm}^{-1}$ & & type & $\mathrm{f}$ & $\mathrm{Je}$ \\
\hline 4901.627 & 100 & 20395.69 & $20406_{s / 2}^{\circ}-40802_{7 / 2}$ & so & 0.22 & 0.98 & 4828.966 & 100 & 20702.58 & $16456_{17 / 2}-37159^{\circ}{ }_{15 / 2}$ & so & 0.79 & 0.36 \\
\hline 4901.165 & 1 & 20397.62 & $22559^{\circ}{ }_{11 / 2}-42957_{11 / 2}^{1 / 2}$ & & & & 4828.797 & 15 & 20703.31 & & $\mathrm{~T}$ & 1.16 & \\
\hline 4900.993 & 5 & 20398.33 & $24348_{9 / 2}-44747^{\circ}{ }_{11 / 2}$ & & & & 4828.066 & 40 & 20706.44 & $17454_{13 / 2}-38161^{\circ}{ }_{11 / 2}$ & $\mathrm{~T}$ & 1.14 & \\
\hline 4900.746 & 5 & 20399.36 & $21799^{\circ}{ }_{3 / 2}-42198_{5 / 2}$ & & & & 4827.430 & 10 & 20709.17 & $18853_{11 / 2}-39563^{\circ}{ }_{11 / 2}$ & & & \\
\hline 4900.545 & 5 & 20400.20 & $22468^{\circ}{ }_{11 / 2}-42868_{11 / 2}$ & & & & 4827.312 & 4 & 20709.68 & $24348_{9 / 2}-45058_{11 / 2}^{\circ}$ & & & \\
\hline 4900.190 & 8 & 20401.68 & $22468^{\circ}{ }_{11 / 2}-42869_{13 / 2}$ & & & & 4825.230 & 1 & 20718.61 & $18990_{11 / 2}-39709^{\circ}{ }_{11 / 2}$ & & & \\
\hline 4900.120 & 10 & 20401.97 & & & & & 4824.465 & 1 & 20721.90 & $24348_{9 / 2}^{/ 2}-45070_{9 / 2}^{\circ}$ & & & \\
\hline 4899.680 & 5 & 20403.80 & $17454_{13 / 2}-37858^{\circ}{ }_{15 / 2}$ & & & & 4822.592 & 2 & 20729.94 & $17752_{5 / 2}-38482^{\circ}{ }_{7 / 2}$ & & & \\
\hline 4897.535 & 1 & 20412.74 & $21120_{z / 2}-41533^{\circ / 2}$ & & & & 4821.597 & 20 & 20734.22 & $17613_{9 / 2}-38347^{\circ}{ }_{11 / 2}$ & & & \\
\hline 4896.150 & 10 & 20418.51 & & & & & 4819.793 & 10 & 20741.98 & $15587_{11 / 2}-36329^{\circ}{ }_{11 / 2}$ & & & \\
\hline 4895.668 & 10 & 20420.52 & $23335^{\circ}{ }_{7 / 2}-43755_{9 / 2}$ & & & & 4818.200 & 2 & 20748.84 & $23941_{9 / 2}^{\circ}-44689_{9 / 2}$ & & & \\
\hline 4894.534 & 4 & 20425.25 & $17343_{\tau^{2} / 2}-37768_{9 / 2}^{\circ}$ & & & & 4817.839 & 10 & 20750.40 & & $\mathrm{~T}$ & 1.35 & \\
\hline 4894.233 & 3 & 20426.51 & & & & & 4817.139 & 1 & 20753.41 & $22791_{7 / 2}-43544_{9 / 2}^{\circ}$ & & & \\
\hline 4893.436 & 1 & 20429.83 & & & & & 4816.387 & 1 & 20756.65 & $24957^{\circ}{ }_{11 / 2}-45714_{11 / 2}$ & & & \\
\hline 4891.670 & 2 & 20437.21 & $24418_{s / 2}-44855_{s / 2}^{\circ}$ & & & & 4815.403 & 3 & 20760.89 & $21120_{7 / 2}^{1 / 2}-41881_{9 / 2}^{\circ}$ & & & \\
\hline 4891.640 & 8 & 20437.33 & & & & & 4813.496 & 150 & 20769.12 & & so & 0.64 & 0.37 \\
\hline 4890.166 & 2 & 20443.49 & $24246^{\circ}{ }_{7 / 2}-44689_{9 / 2}$ & & & & 4812.996 & 1 & 20771.27 & $17613_{9 / 2}-38384_{9 / 2}^{\circ}$ & & & \\
\hline " & & & $23873_{7 / 2}-44316^{\circ}$ & & & & 4812.238 & 2 & 20774.55 & $23431_{5 / 2}^{\circ / 2}-44206_{7 / 2}$ & & & \\
\hline 4889.425 & 20 & 20446.59 & & $\mathrm{~T}$ & 0.96 & & 4811.650 & 50 & 20777.08 & $22419_{9 / 2}^{\circ}-43196_{11 / 2}^{\circ}$ & & & \\
\hline 4887.660 & $40 h$ & 20453.98 & & & & & 4809.756 & 7 & 20785.27 & $19748_{9 / 2}-40533_{7 / 2}^{\circ}$ & & & \\
\hline 4887.346 & 10 & 20455.29 & $21120_{7 / 2}-41576_{9 / 2}^{\circ}$ & & & & 4808.679 & 300 & 20789.92 & $15587_{11 / 2}-36377_{9 / 2}^{\circ}$ & SI & 1.40 & 0.16 \\
\hline 4887.140 & 3 & 20456.15 & & & & & 4807.477 & 100 & 20795.12 & $16456_{17 / 2}-37252^{\circ}{ }_{17 / 2}$ & $\Delta \mathrm{J}=0$ & 1.16 & 0.26 \\
\hline 4886.464 & 3 & 20458.98 & $17752_{s / 2}-38211_{7 / 2}^{\circ}$ & & & & 4807.022 & 7 & 20797.09 & $21799^{\circ}{ }_{3 / 2}^{\circ}-42596_{5 / 2}$ & & & \\
\hline 4884.263 & 10 & 20468.20 & $18853_{11 / 2}-39322_{9 / 2}^{\circ}$ & & & & 4806.709 & 2 & 20798.44 & $19548_{\mathrm{s} / 2}^{\circ / 2}-40347_{\mathrm{s} / 2}^{\circ}$ & & & \\
\hline 4883.675 & 200 & 20470.67 & $19132_{3 / 2}-39602^{\circ}{ }_{3 / 2}$ & $\Delta \mathrm{J}=0$ & 0.88 & $1.30 *$ & 4805.502 & 10 & 20803.67 & $21737_{9 / 2}-42541_{11 / 2}^{\circ / 2}$ & so & 0.97 & 0.12 \\
\hline 4879.194 & 300 & 20489.47 & $18990_{11 / 2}-39479^{\circ}{ }_{11 / 2}$ & $\Delta \mathrm{J}=0$ & 1.20 & 0.12 & 4804.273 & 5 & 20808.99 & $23941_{9 / 2}^{\circ}-44750_{7 / 2}$ & & & \\
\hline 4877.022 & 1 & 20498.59 & & & & & 4801.266 & 10 & 20822.02 & & & & \\
\hline 4874.590 & 40 & 20508.82 & $18853_{11 / 2}-39362^{\circ}{ }_{1 / 2}$ & $\Delta \mathrm{J}=0$ & 1.15 & 0.13 & 4799.509 & 30 & 20829.64 & & & & \\
\hline 4874.420 & 3 & 20509.53 & & & & & 4799.297 & 4 & 20830.56 & & & & \\
\hline 4873.819 & 2 & 20512.06 & & & & & 4798.633 & 10 & 20833.45 & $23431_{5 / 2}^{\circ}-44265_{7 / 2}$ & & & \\
\hline 4870.997 & 7 & 20523.95 & $23335^{\circ}{ }_{7 / 2}-43859_{s / 2}$ & & & & 4798.105 & 4 & 20835.74 & $23374_{13 / 2}-44210^{\circ}{ }_{15 / 2}$ & & & \\
\hline 4870.687 & 20 & 20525.25 & $18837_{9 / 2}-39362^{\circ}{ }_{11 / 2}$ & $\mathrm{~T}$ & 1.13 & & 4796.660 & 10 & 20842.01 & $21997_{11 / 2}-42839_{9 / 2}^{\circ}$ & so & 0.98 & 0.09 \\
\hline 4870.367 & 200 & 20526.60 & $19132_{3 / 2}-39658^{\circ}{ }_{s / 2}$ & SI & 1.09 & 0.13 & 4796.138 & 3 & 20844.28 & $21737_{9 / 2}-42581_{11 / 2}^{\circ}$ & & & \\
\hline 4869.839 & 50 & 20528.83 & & So & 0.80 & 0.35 & 4795.343 & 10 & 20847.74 & $19748_{9 / 2}-40596^{\circ}{ }_{7 / 2}^{\circ / 2}$ & so & 1.22 & 0.07 \\
\hline 4868.372 & 1 & 20535.01 & $23781_{9 / 2}-44316_{9 / 2}^{\circ}$ & & & & 4793.628 & 1 & 20855.20 & $18853_{11 / 2}-39709_{11 / 2}^{\circ}$ & & & \\
\hline 4867.812 & 4 & 20537.37 & $22419^{\circ}{ }_{9 / 2}^{\circ}-42957_{11 / 2}$ & & & & 4793.360 & 4 & 20856.36 & $21737_{9 / 2}-42594_{9 / 2}^{\circ}$ & & & \\
\hline 4866.270 & 50 & 20543.88 & $21997_{11 / 2}-42541_{11 / 2}^{\circ}$ & $\Delta \mathrm{J}=0$ & 1.08 & 0.17 & 4792.393 & 60 & 20860.57 & & So & 0.54 & 0.50 \\
\hline 4865.392 & 10 & 20547.59 & $17613_{9 / 2}-38161_{11 / 2}^{\circ}$ & So & 0.87 & 0.27 & 4791.915 & 2 & 20862.65 & & & & \\
\hline 4865.000 & 3 & 20549.24 & $23431_{s / 2}^{\circ / 2}-43981_{7 / 2}$ & & & & 4790.632 & 30 & 20868.24 & $17343_{7 / 2}-38211^{\circ}{ }_{7 / 2}$ & $\Delta \mathrm{J}=0$ & 1.10 & 0.54 \\
\hline 4863.571 & $2 d$ & 20555.28 & & & & & 4789.659 & 4 & 20872.48 & $22419_{9 / 2}^{\circ}-43292_{9 / 2}$ & & & \\
\hline 4862.934 & 30 & 20557.97 & $17454_{13 / 2}-38012^{\circ}{ }_{13 / 2}$ & $\Delta \mathrm{J}=0$ & 1.12 & 0.26 & 4787.429 & 5 & 20882.20 & & & & \\
\hline 4861.867 & 4 & 20562.49 & $16957_{\gamma / 2}-37519^{\circ}{ }^{\circ}$ & & & & 4786.704 & 2 & 20885.36 & $17613_{9 / 2}-38499_{9 / 2}^{\circ}$ & & & \\
\hline 4861.495 & 2 & 20564.06 & $21997_{11 / 2}-42561_{9 / 2}^{\circ}$ & & & & 4786.210 & 2 & 20887.52 & & & & \\
\hline 4860.398 & 1 & 20568.70 & $19753_{7 / 2}-40322_{9 / 2}^{\circ}$ & & & & 4785.335 & 2 & 20891.34 & & & & \\
\hline 4859.473 & 30 & 20572.62 & $18990_{11 / 2}^{1 / 2}-39563^{\circ}{ }_{11 / 2}$ & $\Delta \mathrm{J}=0$ & 1.15 & 0.57 & 4784.179 & 2 & 20896.39 & & & & \\
\hline 4859.310 & 4 & 20573.31 & & & & & 4783.116 & 4 & 20901.03 & $23873_{7 / 2}-44774_{9 / 2}^{\circ}$ & & & \\
\hline 4857.658 & 1 & 20580.30 & $18853_{11 / 2}-39434_{13 / 2}^{\circ}$ & & & & 4782.501 & 1 & 20903.72 & $23335_{7 / 2}^{\circ}-44238_{9 / 2}$ & & & \\
\hline 4856.670 & 20 & 20584.49 & $21997_{11 / 2}-42581^{\circ}{ }_{11 / 2}$ & $\Delta \mathrm{J}=0$ & 1.09 & 0.42 & 4782.312 & $5 d$ & 20904.54 & $18837_{9 / 2}-39741_{9 / 2}^{\circ}$ & & & \\
\hline 4856.356 & 10 & 20585.82 & & & & & 4781.460 & 1 & 20908.27 & $23335^{\circ}{ }_{7 / 2}-44243_{s / 2}$ & & & \\
\hline 4855.758 & 2 & 20588.36 & $23941_{9 / 2}^{\circ}-44529_{7 / 2}$ & & & & 4780.716 & 10 & 20911.52 & $17752_{5 / 2}^{/ 2}-38664_{7 / 2}^{\circ}$ & so & 0.65 & 0.38 \\
\hline 4854.566 & 3 & 20593.41 & $19753_{7 / 2}-40347^{\circ}{ }_{s / 2}$ & & & & 4780.480 & 1 & 20912.56 & $21997_{11 / 2}-42910^{\circ}{ }_{13 / 2}$ & & & \\
\hline 4853.825 & 2 & 20596.55 & $21997_{11 / 2}-42594_{9 / 2}^{\circ / 2}$ & & & & 4780.020 & 1 & 20914.57 & $19748_{9 / 2}-40663_{11 / 2}^{\circ}$ & & & \\
\hline 4853.495 & 30 & 20597.96 & $17613_{9 / 2}^{/ 2}-38211^{\circ}{ }_{7 / 2}$ & SI & 1.25 & 0.05 & 4779.689 & 20 & 20916.02 & $17613_{9 / 2}-38529^{\circ}{ }_{11 / 2}$ & so & 1.03 & 0.10 \\
\hline 4851.755 & 100 & 20605.34 & $19132_{3 / 2}-39737^{\circ}{ }_{1 / 2}$ & SI & 0.92 & 0.04 & 4776.461 & 1 & 20930.15 & $23335^{\circ}{ }_{7 / 2}-44265_{\tau / 2}$ & & & \\
\hline 4850.415 & 3 & 20611.03 & & & & & 4775.924 & 2 & 20932.50 & $23374_{13 / 2}-44307^{\circ}{ }_{13 / 2}$ & & & \\
\hline 4847.925 & 70 & 20621.62 & & SI & 1.47 & 0.36 & 4775.465 & 20 & 20934.52 & $22902^{\circ}{ }_{13 / 2}-43836_{13 / 2}$ & $\Delta \mathrm{J}=0$ & 1.16 & 0.26 \\
\hline 4847.573 & 2 & 20623.12 & $23335_{\tau_{1 / 2}}^{\circ}-43958_{s_{/ 2}}$ & & & & 4774.162 & $40 h$ & 20940.23 & & & & \\
\hline 4846.887 & 40 & 20626.04 & $18853_{11 / 2}-39479^{\circ}{ }_{11 / 2}$ & $\Delta \mathrm{J}=0$ & 1.18 & 0.28 & 4771.340 & 2 & 20952.61 & $15271_{15 / 2}-36223^{\circ}{ }_{13 / 2}$ & & & \\
\hline 4845.544 & 3 & 20631.75 & & & & & 4769.035 & 2 & 20962.74 & & & & \\
\hline 4844.591 & 50 & 20635.81 & $15587_{11 / 2}-36223^{\circ}{ }_{13 / 2}$ & So & 0.38 & 0.76 & 4768.447 & 6 & 20965.33 & $23781_{9 / 2}-44747_{11 / 2}^{\circ}$ & $\mathrm{T}$ & 1.19 & \\
\hline 4844.237 & 2 & 20637.32 & $22559^{\circ}{ }_{11 / 2}-43196_{11 / 2}$ & & & & 4767.328 & 10 & 20970.25 & & so & 0.40 & 0.58 \\
\hline 4842.935 & 10 & 20642.87 & $18837_{9 / 2}-39480^{\circ}{ }_{7 / 2}$ & $\mathrm{~T}$ & 1.04 & 0.08 & 4766.965 & 2 & 20971.84 & $21161_{s / 2}-42133_{7 / 2}^{\circ}$ & & & \\
\hline 4841.066 & 1 & 20650.84 & $18837_{9 / 2}-39488_{9 / 2}^{\circ}$ & & & & 4766.752 & 1 & 20972.78 & $22742^{\circ}{ }_{13 / 2}-43715_{11 / 2}$ & & & \\
\hline 4837.653 & 1 & 20665.41 & $21120_{7 / 2}-41786_{9 / 2}^{\circ}$ & & & & 4761.714 & 1 & 20994.97 & $23873_{7 / 2}-44868_{9 / 2}^{\circ}$ & & & \\
\hline 4835.903 & 5 & 20672.89 & $24246^{\circ}{ }_{7 / 2}-44919_{9 / 2}$ & & & & 4760.460 & 3 & 21000.50 & & & & \\
\hline 4835.754 & 300 & 20673.52 & $13119_{9 / 2}-33793^{\circ}{ }_{11 / 2}$ & So & 1.01 & 0.24 & 4759.905 & 500 & 21002.95 & $15271_{13 / 2}-36273^{\circ}{ }_{15 / 2}$ & $\Delta \mathrm{J}=0$ & 1.10 & 0.33 \\
\hline 4834.607 & 2 & 20678.43 & $22419_{9 / 2}^{\circ}-43098_{7 / 2}$ & & & & 4759.587 & 1 & 21004.35 & $21737_{9 / 2}-42742^{\circ}{ }_{7 / 2}$ & & & \\
\hline 4832.400 & 2 & 20687.87 & $15271_{15 / 2}-35958^{\circ}{ }_{13 / 2}$ & & & & 4757.756 & 2 & 21012.44 & $21120_{\eta_{2}}-42133^{\circ}{ }_{7 / 2}$ & & & \\
\hline 4831.930 & 20 & 20689.88 & & $\mathrm{~T}$ & 1.10 & & 4757.295 & 1 & 21014.47 & & & & \\
\hline 4830.590 & 15 & 20695.62 & $22419_{9 / 2}^{\circ}-43115_{9 / 2}$ & & & & 4757.095 & 7 & 21015.36 & $19748_{9 / 2}-40763_{7 / 2}^{\circ}$ & SI & 1.65 & 0.36 \\
\hline
\end{tabular}


APPENDiX - Spectral Lines of Neutral Thulium - Continued

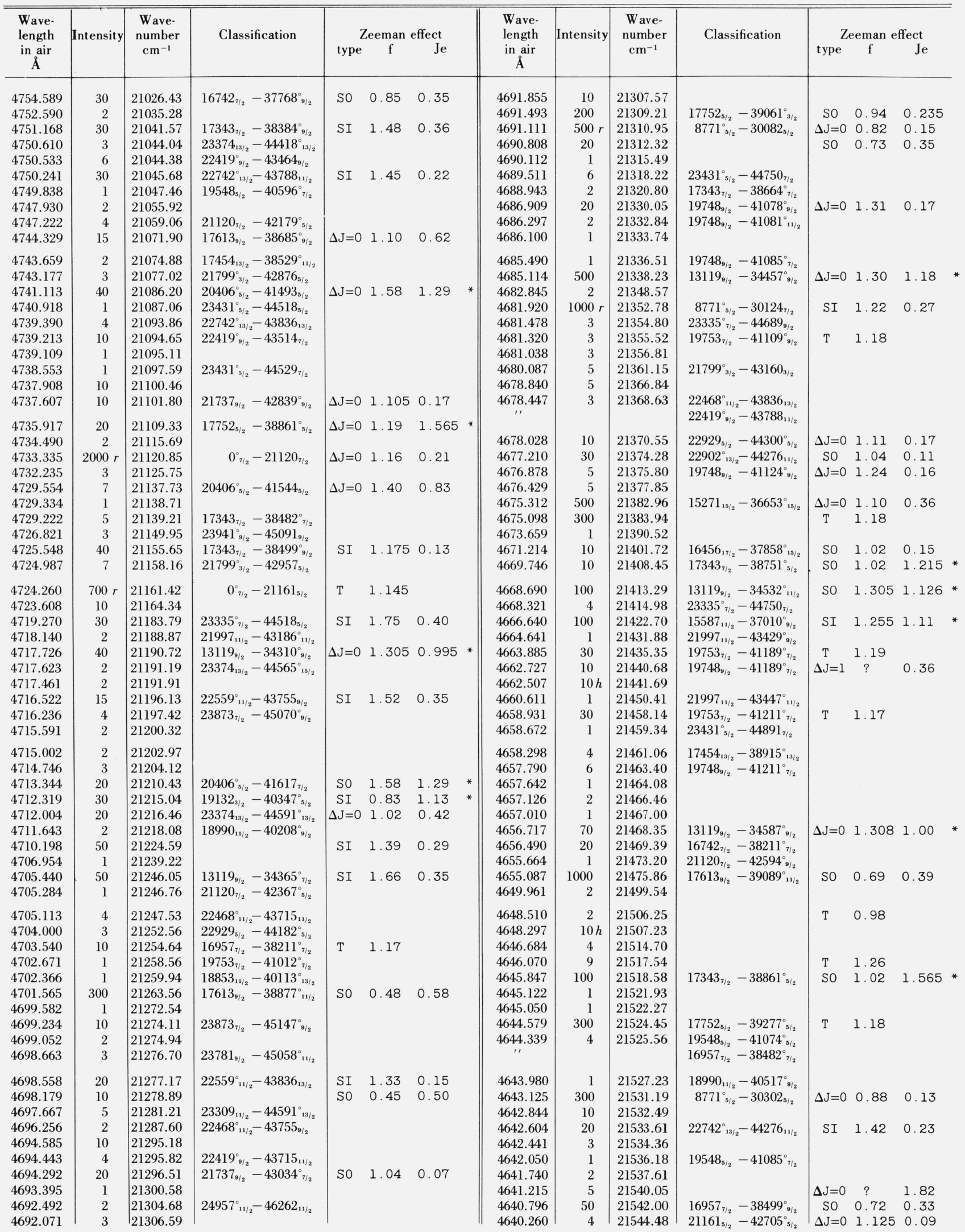


APPENDIX - Spectral Lines of Neutral Thulium-Continued

\begin{tabular}{|c|c|c|c|c|c|c|c|c|c|c|c|c|c|c|}
\hline \multirow{2}{*}{$\begin{array}{c}\text { Wave- } \\
\text { length } \\
\text { in air } \\
\AA\end{array}$} & \multirow[t]{2}{*}{ Intensity } & \multirow{2}{*}{$\begin{array}{c}\text { Wave- } \\
\text { number } \\
\text { cm }^{-1}\end{array}$} & \multirow[t]{2}{*}{ Classification } & \multirow{2}{*}{\multicolumn{4}{|c|}{ Zeeman effect }} & \multirow{2}{*}{$\begin{array}{c}\text { Wave- } \\
\text { length } \\
\text { in air } \\
\AA\end{array}$} & Intensity & $\begin{array}{l}\text { W ave- } \\
\text { number }\end{array}$ & Classification & & eeman ef & \\
\hline & & & & & & & & & & $\mathrm{cm}^{-1}$ & & type & $\mathrm{f}$ & \\
\hline 4638.932 & 3 & 21550.65 & & $\Delta \mathrm{J}=0$ & 1.22 & 0.18 & & 4581.006 & 4 & 21823.15 & & & & \\
\hline 4638.287 & 2 & 21553.65 & $22929_{5 / 2}-44483_{3 / 2}^{\circ}$ & & & & & 4580.460 & 2 & 21825.75 & $18837_{9 / 2}-40663^{\circ}{ }_{11 / 2}$ & & & \\
\hline 4637.767 & 5 & 21556.06 & $23335^{\circ}{ }_{\tau / 2}^{\circ}-44891_{\tau / 2}^{1 / 2}$ & & & & & 4580.067 & 3 & 21827.63 & $19748_{9 / 2}-41576_{9 / 2}^{\circ}$ & & & \\
\hline 4636.633 & 10 & 21561.34 & $22419_{9 / 2}^{\circ}-43981_{7 / 2}$ & & & & & 4579.438 & 10 & 21830.62 & $17613_{9 / 2}-39444_{9 / 2}^{\circ}$ & $\Delta \mathrm{J}=0$ & 1.15 & 0.25 \\
\hline 4635.795 & $5 h$ & 21565.23 & & & & & & 4578.820 & $10 h$ & 21833.57 & & & & \\
\hline 4633.960 & 300 & 21573.77 & $17613_{9_{/ 2}}-39187^{\circ}{ }_{7 / 2}$ & SO & 0.61 & 0.58 & & 4576.609 & 80 & 21844.12 & $17343_{\tau_{/ 2}}-39187^{\circ}{ }_{7 / 2}$ & $\Delta \mathrm{J}=0$ & 1.022 & 1.336 \\
\hline 4632.490 & 5 & 21580.62 & $21161_{5 / 2}^{7 / 2}-42742^{\circ}{ }_{7 / 2}^{\circ}$ & & & & & 4576.307 & 20 & 21845.56 & $22419_{9 / 2}^{\circ}-44265_{7 / 2}$ & So & 0.43 & 0.49 \\
\hline 4632.349 & 4 & 21581.28 & & & & & & 4575.555 & 20 & 21849.15 & $17613_{9 / 2}-39462^{\circ}{ }_{7 / 2}$ & & & \\
\hline 4631.538 & 10 & 21585.06 & $21120_{7 / 2}-42705_{5 / 2}^{\circ}$ & SI & 1.32 & 0.16 & & 4575.322 & 30 & 21850.26 & $17752_{5 / 2}-39602_{3 / 2}^{\circ}$ & So & 1.01 & 0.165 \\
\hline 4631.104 & 1 & 21587.08 & & & & & & 4573.981 & 2 & 21856.67 & $22419_{9 / 2}^{\circ}-44276_{11 / 2}^{\circ}$ & & & \\
\hline 4626.968 & 200 & 21606.37 & $17613_{9 / 2}-39220_{9 / 2}^{\circ}$ & $\Delta \mathrm{J}=0$ & 1.15 & 0.31 & & 4573.702 & 20 & 21858.00 & $22559^{\circ}{ }_{11 / 2}-44417_{11 / 2}$ & $\Delta \mathrm{J}=0$ & 1.16 & 0.165 \\
\hline 4625.576 & 40 & 21612.88 & $22902^{\circ}{ }_{13 / 2}-44515_{13 / 2}$ & $\Delta \mathrm{J}=0$ & 1.13 & 0.14 & & 4573.190 & 1 & 21860.45 & & & & \\
\hline 4625.245 & 1 & 21614.42 & $21120_{z^{2} / 2}-42735_{\mathrm{s} / 2}^{\circ}$ & & & & & 4572.197 & 5 & 21865.20 & $18990_{11 / 2}-40855_{9 / 2}^{\circ}$ & & & \\
\hline 4624.402 & 30 & 21618.36 & & $\Delta J=0$ & 1.03 & 0.54 & & 4571.900 & 80 & 21866.62 & $17613_{9 / 2}-39480_{7 / 2}^{\circ}$ & SI & 1.25 & 0.06 \\
\hline 4623.800 & 3 & 21621.18 & $21120_{7 / 2}-42742_{7 / 2}^{\circ}$ & $\Delta \mathrm{J}=0$ & 1.18 & 0.09 & & 4571.225 & 30 & 21869.85 & & SI & 1.40 & 0.125 \\
\hline 4621.716 & 300 & 21630.93 & $17613_{9 / 2}-39244_{9 / 2}^{\circ}$ & $\Delta \mathrm{J}=0$ & 1.19 & 1.04 & * & 4570.617 & 10 & 21872.75 & $21161_{5 / 2}-43034^{\circ}{ }_{7 / 2}$ & $\mathrm{~T}$ & 1.18 & 0.02 \\
\hline 4620.989 & 50 & 21634.33 & $17752_{5 / 2}-39386_{5 / 2}^{\circ}$ & $\Delta \mathrm{J}=0$ & 1.07 & $0.5]$ & & 4570.240 & 50 & 21874.56 & $17613_{9_{/ 2}}-39488_{9 / 2}^{\circ}$ & $\Delta \mathrm{J}=0$ & 1.15 & 0.36 \\
\hline 4619.204 & 4 & 21642.69 & $16742_{7 / 2}-38384_{9 / 2}^{\circ}$ & & & & & 4569.803 & 10 & 21876.65 & $19548_{5 / 2}-41425^{\circ}{ }_{7 / 2}$ & & & \\
\hline 4615.296 & 20 & 21661.02 & $17752_{5 / 2}-39413^{\circ}{ }_{7 / 2}$ & so & 1.11 & 0.06 & & & & & $17343_{7 / 2}-39220_{9 / 2}^{\circ}$ & & & \\
\hline 4615.101 & 3 & 21661.93 & & & & & & 4567.329 & 3 & 21888.50 & $15271_{15 / 2}-37159^{\circ}{ }_{15 / 2}$ & & & \\
\hline 4614.696 & 10 & 21663.83 & $18853_{11 / 2}-40517_{9 / 2}^{\circ}$ & & & & & 4566.532 & 5 & 21892.32 & $19753_{7 / 2}-41646^{\circ}{ }_{7 / 2}$ & & & \\
\hline 4613.967 & 200 & 21667.25 & $17752_{5 / 2}-39419_{3 / 2}^{\circ}$ & SI & 1.186 & 0.916 & $*$ & 4565.432 & 20 & 21897.59 & $19748_{9 / 2}-41646^{\circ}{ }_{7 / 2}$ & & & \\
\hline 4613.112 & 1 & 21671.27 & $21737_{9 / 2}-43408^{\circ}{ }_{11 / 2}$ & & & & & 4565.053 & 2 & 21899.41 & & & & \\
\hline 4613.035 & 5 & 21671.63 & $19753_{7 / 2}-41425_{7 / 2}^{\circ}$ & & & & & 4564.676 & 400 & 21901.22 & $17343_{7_{/ 2}}-39244_{9 / 2}^{\circ}$ & $\mathrm{T}$ & 1.02 & \\
\hline 4612.380 & 7 & 21674.71 & $22742^{\circ}{ }_{13 / 2}-44417_{11 / 2}$ & SI & 1.42 & 0.21 & & 4564.260 & 3 & 21903.22 & & & & \\
\hline 4612.094 & 1 & 21676.05 & & & & & & 4563.892 & 20 & 21904.98 & $16957_{z / 2}-38861^{\circ}{ }^{\circ} / 2$ & & & \\
\hline 4611.197 & 1 & 21680.27 & $18837_{9 / 2}-40517_{9 / 2}^{\circ}$ & & & & & 4563.642 & 20 & 21906.18 & $17752_{5 / 2}-39658_{5 / 2}^{\circ}$ & & & \\
\hline 4610.595 & 4 & 21683.10 & $18693_{15 / 2}-40376^{\circ}{ }_{13 / 2}$ & & & & & 4561.521 & 2 & 21916.37 & & & & \\
\hline 4610.470 & 2 & 21683.69 & $23374_{13 / 2}-45058^{\circ}{ }_{11 / 2}$ & & & & & 4560.137 & 3 & 21923.02 & & & & \\
\hline 4609.354 & 50 & 21688.94 & $15587_{11 / 2}-37276^{\circ}{ }_{11 / 2}$ & $\mathrm{~T}$ & 1.25 & & & 4559.300 & 2 & 21927.05 & $18853_{11 / 2}-40780^{\circ}{ }_{13 / 2}$ & & & \\
\hline 4608.913 & 2 & 21691.01 & & & & & & 4558.335 & 1 & 21931.69 & $21120_{7 / 2}-43052^{\circ}{ }_{7 / 2}$ & & & \\
\hline 4608.895 & 3 & 21691.10 & $21737_{9 / 2}-43428^{\circ}{ }_{7 / 2}$ & $\mathrm{~T}$ & 1.15 & 0.06 & & 4557.917 & 3 & 21933.70 & $17343_{7 / 2}-39277^{\circ}{ }_{5 / 2}$ & & & \\
\hline 4608.795 & 1 & 21691.57 & & & & & & 4557.633 & 200 & 21935.07 & $13119_{9 / 2}-35054_{9 / 2}^{\circ}$ & $\Delta \mathrm{J}=0$ & 1.29 & 0.115 \\
\hline 4607.967 & 1 & 21695.47 & & & & & & 4556.849 & 3 & 21938.84 & $19748_{9 / 2}-41687^{\circ}{ }_{11 / 2}$ & & & \\
\hline 4607.453 & 2 & 21697.89 & & & & & & 4555.923 & 3 & 21943.30 & $16742_{7 / 2}-38685_{9 / 2}^{\circ}$ & & & \\
\hline 4607.330 & 2 & 21698.47 & & & & & & 4554.650 & 10 & 21949.43 & $22468^{\circ}{ }_{11 / 2}-44417_{11 / 2}$ & $\Delta \mathrm{J}=0$ & 1.20 & 0.25 \\
\hline 4607.036 & 2 & 21699.85 & $23431_{\mathrm{s} / 2}^{\circ}-45131_{7 / 2}$ & & & & & 4554.065 & 2 & 21952.25 & $21161_{5 / 2}-43113_{5 / 2}^{\circ}$ & & & \\
\hline 4607.018 & 4 & 21699.94 & & & & & & 4553.677 & 30 & 21954.12 & & SI & 1.64 & 0.335 \\
\hline 4606.608 & 10 & 21701.87 & & & & & & 4553.391 & 20 & 21955.50 & $22559^{\circ}{ }_{11 / 2}-44515_{13 / 2}$ & So & 0.92 & 0.18 \\
\hline 4605.660 & 3 & 21706.33 & & & & & & 4552.226 & 7 & 21961.12 & $22419_{9 / 2}^{\circ}-44380_{9 / 2}$ & & & \\
\hline 4605.231 & 40 & 21708.36 & $17613_{9 / 2}-39322_{9 / 2}^{\circ}$ & $\Delta \mathrm{J}=0$ & 1.17 & 0.18 & & 4549.069 & 2 & 21976.36 & $19548_{5 / 2}-41525_{7 / 2}^{\circ}$ & & & \\
\hline 4604.850 & 300 & 21710.15 & $17752_{5 / 2}-39462_{7 / 2}^{\circ}$ & SO & 0.98 & 0.14 & & 4548.597 & 1000 & 21978.64 & $17343_{7 / 2}-39322_{9 / 2}^{\circ}$ & SI & 1.61 & 0.45 \\
\hline 4603.217 & 60 & 21717.85 & $17752_{5 / 2}-39470^{\circ}{ }_{5 / 2}$ & $\Delta \mathrm{J}=0$ & 1.14 & 0.36 & & 4548.105 & 50 & 21981.02 & $15271_{15 / 2}-37252^{\circ}{ }_{17 / 2}$ & SI & 2.10 & 0.90 \\
\hline 4603.051 & 20 & 21718.64 & $21120_{7 / 2}-42839_{9 / 2}^{\circ}$ & So & 0.69 & 0.38 & & 4547.341 & 5 & 21984.71 & $19548_{5 / 2}-41533_{5 / 2}^{\circ}$ & $\Delta \mathrm{J}=0$ & $?$ & 0.10 \\
\hline 4601.580 & 3 & 21725.58 & & $\Delta J=0$ & 1.05 & 0.38 & & 4545.777 & 2 & 21992.27 & & & & \\
\hline 4601.147 & 10 & 21727.62 & $17752_{5 / 2}-39480^{\circ}{ }_{7 / 2}$ & $\mathrm{~T}$ & 1.15 & 0.03 & & 4544.660 & 10 & 21997.68 & $22419_{9 / 2}^{\circ}-44417_{11 / 2}$ & & & \\
\hline 4599.017 & $2000 r$ & 21737.69 & $0_{7 / 2}^{\circ}-21737_{9 / 2}$ & so & 1.09 & 0.045 & & 4543.870 & 10 & 22001.50 & $21799^{\circ}{ }_{3 / 2}-43800_{3 / 2}$ & & & \\
\hline 4598.225 & 4 & 21741.43 & & so & 0.20 & 0.49 & & 4543.825 & 2 & 22001.72 & $18853_{11 / 2}-40855_{9 / 2}^{\circ}$ & & & \\
\hline 4596.626 & 1000 & 21748.99 & $17613_{9 / 2}-39362^{\circ}{ }_{11 / 2}$ & SO & 0.88 & 0.26 & & 4539.406 & 80 & 22023.14 & $22559^{\circ}{ }_{11 / 2}-44582_{9 / 2}$ & SI & 1.70 & 0.47 \\
\hline 4595.420 & 1 & 21754.70 & & & & & & 4538.645 & 2 & 22026.83 & & & & \\
\hline 4594.979 & 4 & 21756.79 & $16742_{7 / 2}-38499_{9 / 2}^{\circ}$ & so & 0.13 & 0.925 & & 4538.212 & 4 & 22028.93 & $16456_{17 / 2}-38485^{\circ}{ }_{15 / 2}$ & & & \\
\hline 4594.532 & 3 & 21758.91 & $18837_{9 / 2}-40596_{7 / 2}^{\circ}$ & & & & & 4537.442 & 2 & 22032.67 & & & & \\
\hline 4592.677 & 2 & 21767.69 & & $\Delta \mathrm{J}=0$ & 1.15 & 0.51 & & 4536.406 & 2 & 22037.70 & $19748_{9 / 2}-41786_{9 / 2}^{\circ}$ & & & \\
\hline 4591.717 & 4 & 21772.25 & $22742^{\circ}{ }_{13 / 2}-44515_{13 / 2}$ & & & & & 4535.198 & 40 & 22043.57 & $17343_{7 / 2}-39386_{5 / 2}^{\circ}$ & SI & 1.08 & 0.06 \\
\hline 4590.694 & 5 & 21777.10 & $19548_{5 / 2}-41325^{\circ}{ }_{5 / 2}^{\circ}$ & & & & & 4534.501 & 2 & 22046.96 & $22468^{\circ}{ }_{11 / 2}-44515_{13 / 2}$ & & & \\
\hline 4590.649 & 40 & 21777.31 & $15587_{11 / 2}-37365^{\circ}{ }_{13 / 2}$ & so & 0.91 & 0.29 & & 4532.150 & 300 & 22058.40 & $15587_{11 / 2}-37646^{\circ}{ }_{13 / 2}$ & so & 0.82 & 0.44 \\
\hline 4589.729 & 10 & 21781.68 & $21120_{7 / 2}-42902^{\circ}{ }_{5 / 2}$ & SI & 1.50 & 0.33 & & 4531.130 & 2 & 22063.36 & & & & \\
\hline 4588.684 & 6 & 21786.64 & $22419_{9 / 2}^{\circ}-44206_{7 / 2}^{\circ}$ & & & & & 4529.910 & 5 & 22069.31 & $21161_{5 / 2}-43230_{3 / 2}^{\circ}$ & & & \\
\hline 4588.423 & 1 & 21787.88 & & & & & & 4529.745 & 2 & 22070.11 & $15587_{11 / 2}-37657^{\circ}{ }_{11 / 2}$ & & & \\
\hline 4588.067 & 50 & 21789.57 & $17752_{5 / 2}-39542^{\circ}{ }_{\tau / 2}$ & So & 0.73 & 0.32 & & 4526.043 & 30 & 22088.16 & $18990_{11 / 2}-41078_{9 / 2}^{\circ}$ & so & 0.68 & 0.525 \\
\hline 4587.870 & 1 & 21790.50 & $18990_{11 / 2}-40780^{\circ}{ }_{13 / 2}$ & & & & & 4525.469 & 2 & 22090.96 & $18990_{11 / 2}-41081^{\circ}{ }_{11 / 2}$ & & & \\
\hline 4587.567 & 10 & 21791.94 & $20406_{5 / 2}^{\circ}-42198_{5 / 2}$ & $\Delta \mathrm{J}=0$ & 1.58 & 1.255 & $*$ & 4525.034 & 10 & 22093.09 & $19748_{9 / 2}-41841_{7 / 2}^{\circ}$ & & & \\
\hline 4586.967 & 20 & 21794.79 & $16957_{7 / 2}-38751_{5 / 2}^{\circ}$ & so & 1.06 & 0.11 & & 4524.821 & 200 & 22094.13 & $15271_{15 / 2}-37365^{\circ}{ }_{13 / 2}$ & so & 0.29 & 0.78 \\
\hline 4586.062 & 1 & 21799.09 & & & & & & 4524.567 & 3 & 22095.37 & $17613_{9 / 2}-39709^{\circ}{ }_{11 / 2}$ & & & \\
\hline 4584.535 & 6 & 21806.35 & $19132_{3 / 2}-40938_{5 / 2}^{\circ}$ & SI & 1.54 & 0.33 & & 4524.168 & 3 & 22097.32 & $19548_{5 / 2}-41646^{\circ}{ }_{7 / 2}^{1 / 2}$ & & & \\
\hline 4584.110 & 5 & 21808.37 & $22468^{\circ}{ }_{11 / 2}-44276_{11 / 2}$ & & & & & 4523.430 & 20 & 22100.92 & $17343_{\tau^{\prime} / 2}-39444_{9 / 2}^{\circ}$ & & & \\
\hline 4583.914 & 2 & 21809.31 & $18853_{11 / 2}-40663^{\circ}{ }_{11 / 2}$ & & & & & 4521.643 & 10 & 22109.65 & $22419_{9 / 2}^{\circ}-44529_{7 / 2}$ & so & 0.83 & 0.07 \\
\hline 4582.842 & 100 & 21814.41 & $8771_{5 / 2}^{\circ}-30585_{3 / 2}$ & so & 0.855 & $1.085 *$ & * & 4520.632 & 20 & 22114.60 & $22468^{\circ}{ }_{11 / 2}-44582_{9 / 2}$ & SI & 1.85 & 0.56 \\
\hline 4581.856 & 4 & 21819.10 & $22419_{9 / 2}^{\circ}-44238_{9 / 2}^{\circ}$ & & & & & 4519.734 & 20 & 22118.99 & $18990_{11 / 2}-41109^{\circ}$ & $\mathrm{T}$ & 1.20 & \\
\hline 4581.547 & 1 & 21820.57 & $19748_{9 / 2}-41569^{\circ 1 / 2}$ & & & & & 4519.595 & 2000 & 22119.67 & $16742_{\tau_{/ 2}}-38861_{5 / 2}^{\circ}$ & So & 1.32 & 1.57 \\
\hline
\end{tabular}


APPENDIX - Spectral Lines of Neutral Thulium - Continued

\begin{tabular}{|c|c|c|c|c|c|c|c|c|c|c|c|c|c|c|}
\hline \multirow{2}{*}{$\begin{array}{c}\text { Wave- } \\
\text { length } \\
\text { in air } \\
\AA\end{array}$} & \multirow[t]{2}{*}{ Intensity } & \multirow{2}{*}{$\begin{array}{l}\text { Wave- } \\
\text { number } \\
\mathrm{cm}^{-1}\end{array}$} & \multirow[t]{2}{*}{ Classification } & \multirow{2}{*}{\multicolumn{3}{|c|}{\begin{tabular}{l}
\multicolumn{3}{c}{ Zeeman effect } \\
f $\quad J e$
\end{tabular}}} & \multirow{2}{*}{$\begin{array}{c}\text { Wave- } \\
\text { length } \\
\text { in air } \\
\AA\end{array}$} & Intensity & $\begin{array}{c}\text { Wave- } \\
\text { number }\end{array}$ & Classification & & Ceeman ef & ffect & \\
\hline & & & & & & & & & $\mathrm{cm}^{-1}$ & & type & $\mathrm{f}$ & $\mathrm{Je}$ & \\
\hline 4518.350 & 5 & 22125.77 & $19748_{9 / 2}-41874^{\circ}{ }_{11 / 2}$ & & & & 4463.465 & 2 & 22397.83 & & & & & \\
\hline 4517.84 .6 & 70 & 22128.24 & $17613_{9 / 2}-39741_{9 / 2}^{\circ}$ & $\Delta \mathrm{J}=0$ & $?$ & 0.34 & 4463.328 & 3 & 22398.52 & $17343_{7 / 2}-39741_{9 / 2}^{\circ}$ & & & & \\
\hline 4517.400 & 4 & 22130.42 & $22559^{\circ}{ }_{11 / 2}-44689_{9 / 2}$ & & & & 4462.770 & 20 & 22401.32 & $19132_{3 / 2}-41533_{5 / 2}^{\circ}$ & SI & 1.24 & 0.22 & \\
\hline 4517.209 & 1 & 22131.36 & & & & & 4461.310 & $3 h$ & 22408.65 & & & & & \\
\hline 4516.834 & 2 & 22133.19 & $19748_{9 / 2}-41881_{9 / 2}^{\circ}$ & & & & 4459.986 & 100 & 22415.30 & & $\mathrm{~T}$ & 0.895 & 0.05 & \\
\hline 4516.684 & 40 & 22133.93 & $18990_{11 / 2}-41124_{9 / 2}^{\circ}$ & So & 1.12 & 0.09 & 4459.661 & 5 & 22416.94 & & & & & \\
\hline 4516.205 & 70 & 22136.28 & $20406^{\circ}{ }_{5 / 2}^{/ 2}-42543_{7 / 2}$ & SO & 1.58 & $1.12 *$ & 4459.267 & 3 & 22418.92 & & & & & \\
\hline 4514.453 & 20 & 22144.87 & $17343_{7 / 2}-39488_{9 / 2}^{\circ}$ & SI & 1.33 & 0.25 & 4458.059 & 20 & 22424.99 & $15587_{11 / 2}-38012^{\circ}{ }_{13 / 2}$ & so & 0.31 & 0.825 & \\
\hline 4514.226 & 2 & 22145.98 & & & & & 4457.848 & 20 & 22426.05 & $19753_{7 / 2}-42179^{\circ}{ }_{5 / 2}^{\circ / 2}$ & SI & 1.52 & 0.35 & \\
\hline 4508.318 & 1 & 22175.00 & $18837_{9 / 2}-41012_{7 / 2}^{\circ}$ & & & & 4457.071 & 3 & 22429.96 & $16957_{7 / 2}^{1 / 2}-39386_{5 / 2}^{\circ / 2}$ & & & & \\
\hline 4506.220 & 1 & 22185.33 & & & & & 4454.276 & 2 & 22444.04 & $21799_{3 / 2}^{\circ}-44243_{5 / 2}$ & & & & \\
\hline 4505.342 & 1 & 22189.65 & $20406_{s / 2}^{\circ}-42596_{5 / 2}$ & & & & 4454.033 & 1000 & 22445.26 & $16742_{7 / 2}^{0 / 2}-39187^{\circ}{ }_{7 / 2}$ & $\Delta \mathrm{J}=0$ & 1.33 & 0.05 & \\
\hline 4505.273 & 2 & 22189.96 & & & & & 4453.699 & 3 & 22446.95 & & & & & \\
\hline 4504.859 & 60 & 22192.03 & & $\Delta \mathrm{J}=0$ & 1.17 & 0.335 & 4453.562 & 6 & 22447.64 & $17613_{9 / 2}-40061_{\tau_{7 / 2}}^{\circ}$ & & & & \\
\hline 4504.535 & 20 & 22193.62 & $19132_{3 / 2}-41325_{5 / 2}^{\circ}$ & & & & 4452.978 & 10 & 22450.58 & $19466_{13 / 2}-41917^{\circ}{ }_{13 / 2}$ & $\mathrm{~T}$ & 1.15 & & \\
\hline 4504.350 & 7 & 22194.54 & $19548_{5 / 2}-41743_{3 / 2}^{\circ}$ & SI & 1.06 & 0.08 & 4452.843 & 2 & 22451.26 & $22468^{\circ}{ }_{11 / 2}-44919_{9 / 2}$ & & & & \\
\hline 4503.936 & 2 & 22196.58 & & & & & 4451.774 & 2 & 22456.65 & $16957_{7 / 2}-39413^{\circ / 2}{ }_{7 / 2}$ & & & & \\
\hline 4503.484 & 100 & 22198.80 & $17343_{7 / 2}-39542^{\circ}{ }_{7 / 2}$ & $\Delta \mathrm{J}=0$ & 1.04 & 0.15 & 4450.280 & 2 & 22464.19 & & & & & \\
\hline 4500.986 & 2 & 22211.12 & & & & & 4449.772 & 10 & 22466.76 & & so & 0.76 & 0.24 & \\
\hline 4499.039 & 10 & 22220.74 & $19466_{13 / 2}-41687^{\circ}{ }_{11 / 2}$ & $\mathrm{~T}$ & 1.17 & & 4449.223 & 5 & 22469.53 & $20406_{5 / 2}^{\circ}-42876_{5 / 2}$ & & & & \\
\hline 4498.813 & 7 & 22221.85 & $22468_{11 / 2}^{\circ}-44689_{9 / 2}$ & & & & 4448.435 & 2 & 22473.51 & & & & & \\
\hline 4498.230 & 10 & 22224.73 & $18853_{11 / 2}-41078_{9 / 2}^{\circ}$ & & & & 4448.022 & $2 h$ & 22475.59 & & & & & \\
\hline 4497.131 & 2 & 22230.16 & & & & & 4447.824 & 2 & 22476.59 & & & & & \\
\hline 4497.070 & 5 & 22230.46 & $16957_{\tau / 2}-39187^{\circ}{ }_{7 / 2}$ & & & & 4447.585 & 200 & 22477.80 & $16742_{7 / 2}-39220_{9 / 2}^{\circ}$ & SO & 0.40 & 0.72 & \\
\hline 4496.786 & 2 & 22231.87 & & & & & 4447.357 & 2 & 22478.96 & $22929_{5 / 2}-45408^{\circ}{ }_{7 / 2}$ & & & & \\
\hline 4496.022 & $3 h$ & 22235.65 & & & & & 4446.900 & 2 & 22481.26 & & & & & \\
\hline 4495.226 & 3 & 22239.58 & & & & & 4446.406 & $20 h$ & 22483.76 & & & & & \\
\hline 4494.299 & 20 & 22244.17 & $13119_{9 / 2}-35363_{9 / 2}^{\circ}$ & $\Delta \mathrm{J}=0$ & 1.305 & 0.91 & 4445.712 & 5 & 22487.27 & $16957_{7 / 2}-39444_{9 / 2}^{\circ}$ & & & & \\
\hline 4493.333 & 5 & 22248.95 & & & & & 4444.530 & 50 & 22493.25 & & SI & 1.39 & 0.28 & \\
\hline 4492.270 & 20 & 22254.22 & $17454_{13 / 2}-39709^{\circ}{ }_{11 / 2}$ & So & 0.85 & 0.27 & 4443.287 & 4 & 22499.55 & $22419_{9 / 2}^{\circ}-44919_{9 / 2}$ & & & & \\
\hline 4492.001 & 20 & 22255.55 & $18853_{11 / 2}-41109_{9 / 2}^{\circ}$ & so & 0.98 & 0.18 & 4442.735 & 400 & 22502.34 & $16742_{7 / 2}-39244_{9 / 2}^{\circ}$ & SO & 1.325 & 1.03 & * \\
\hline 4491.731 & $2 h$ & 22256.89 & & & & & 4442.059 & 15 & 22505.76 & $16957_{7 / 2}^{1 / 2}-39462^{\circ}{ }_{7 / 2}$ & & & & \\
\hline 4490.900 & 2 & 22261.01 & $23374_{13 / 2}-45635^{\circ}{ }_{11 / 2}$ & & & & 4440.676 & 2 & 22512.77 & & & & & \\
\hline 4489.618 & 3 & 22267.36 & $21161_{5 / 2}-43428_{7 / 2}^{\circ}$ & & & & 4440.535 & 4 & 22513.49 & $16957_{7 / 2}-39470^{\circ}{ }_{5 / 2}$ & & & & \\
\hline 4489.050 & 10 & 22270.18 & $22419_{9 / 2}^{\circ}-44689_{9 / 2}$ & & & & 4440.346 & 2 & 22514.45 & $25130_{15 / 2}-47644^{\circ}{ }_{13 / 2}$ & & & & \\
\hline 4489.016 & 30 & 22270.35 & $13119_{9 / 2}-35389^{\circ}{ }_{7 / 2}$ & SI & 2.50 & 1.20 & 4438.607 & 10 & 22523.27 & $16957_{7 / 2}-39480^{\circ}{ }_{7 / 2}$ & $\mathrm{~T}$ & 1.17 & & \\
\hline 4488.983 & 20 & 22270.51 & $18853_{11 / 2}-41124_{9 / 2}^{\circ / 2}$ & so & 0.72 & 0.425 & 4438.010 & 2 & 22526.30 & $21737_{9 / 2}-44263^{\circ}{ }_{7 / 2}$ & & & & \\
\hline 4487.642 & 2 & 22277.17 & & & & & 4436.985 & 20 & 22531.50 & $22559^{\circ}{ }_{11 / 2}^{\circ}-45091_{9 / 2}$ & $\Delta \mathrm{J}=1$ & $?$ & 0.49 & \\
\hline 4487.196 & 2 & 22279.38 & $22791_{7 / 2}-45070_{9 / 2}^{\circ}$ & & & & 4436.324 & 100 & 22534.86 & $16742_{7 / 2}-39277^{\circ / 2}{ }_{5 / 2}$ & SI & 1.65 & 0.35 & \\
\hline 4486.140 & 150 & 22284.63 & $17343_{7 / 2}-39628^{\circ}{ }_{7 / 2}^{\circ / 2}$ & $\Delta \mathrm{J}=0$ & 1.015 & 0.135 & 4435.070 & 2 & 22541.23 & & & & & \\
\hline 4485.642 & 5 & 22287.10 & & & & & 4434.296 & 40 & 22545.16 & $20406_{5 / 2}^{\circ}-42952_{7 / 2}$ & so & 1.58 & 1.14 & * \\
\hline 4485.543 & 20 & 22287.59 & $16957_{7 / 2}-39244_{9 / 2}^{\circ}$ & & & & 4431.811 & 2 & 22557.81 & & & & & \\
\hline 4485.435 & 2 & 22288.13 & & & & & 4431.561 & 60 & 22559.08 & & $\mathrm{~T}$ & 0.80 & & \\
\hline 4484.797 & $2 h$ & 22291.30 & & & & & 4431.494 & 2 & 22559.4 .2 & & & & & \\
\hline 4484.499 & 5 & 22292.78 & $19548_{5 / 2}-41841^{\circ}{ }_{7 / 2}$ & & & & 4430.862 & 50 & 22562.64 & $13119_{9 / 2}-35682^{\circ}{ }_{7 / 2}$ & SI & 1.305 & 1.12 & * \\
\hline 4482.959 & 1 & 22300.44 & & & & & 4429.756 & $50 h$ & 22568.27 & & & & & \\
\hline 4482.476 & $10 h$ & 22302.84 & & & & & 4428.759 & $3 w$ & 22573.35 & & & & & \\
\hline 4482.169 & 1 & 22304.37 & & & & & 4428.530 & 1 & 22574.52 & & & & & \\
\hline 4481.054 & 2 & 22309.92 & $21120_{\tau_{/ 2}}-43430_{5 / 2}^{\circ}$ & & & & 4427.710 & 2 & 22578.70 & $18990_{11 / 2}-41569^{\circ}{ }_{11 / 2}$ & & & & \\
\hline 4479.938 & 30 & 22315.48 & $17343_{7 / 2}-39658^{\circ}{ }_{5 / 2}^{\circ}$ & SI & 1.20 & 0.15 & 4427.500 & 4 & 22579.77 & $16742_{7 / 2}-39322^{\circ}{ }_{9 / 2}$ & & & & \\
\hline 4478.815 & 20 & 22321.07 & $23941_{9 / 2}^{\circ}-46262_{11 / 2}$ & so & 0.52 & 0.63 & 4426.442 & 10 & 22585.17 & $16957_{7 / 2}-39542^{\circ}{ }_{7 / 2}$ & & & & \\
\hline 4476.960 & 3 & 22330.32 & $22419_{9 / 2}^{\circ / 2}-44750_{7 / 2}$ & & & & 4426.330 & 100 & 22585.74 & $18990_{11 / 2}-41576_{9 / 2}^{\circ}$ & so & 1.04 & 0.165 & \\
\hline 4476.236 & 20 & 22333.93 & & & & & 4425.958 & 200 & 22587.64 & $15271_{15 / 2}-37858^{\circ}{ }_{15 / 2}$ & $\Delta \mathrm{J}=0$ & 1.16 & 0.90 & \\
\hline 4473.402 & 1 & 22348.08 & & & & & 4425.167 & 2 & 22591.67 & & & & & \\
\hline 4472.816 & 60 & 22351.01 & & & & & 4423.265 & 10 & 22601.39 & & & & & \\
\hline 4471.990 & 40 & 22355.14 & & & & & 4422.305 & 70 & 22606.29 & $18693_{15 / 2}-41299^{\circ}{ }_{17 / 2}$ & so & 0.28 & 0.775 & \\
\hline 4471.788 & 2 & 22356.15 & $22791_{7 / 2}-45147_{9 / 2}^{\circ}$ & & & & 4421.670 & $2 h$ & 22609.54 & & & & & \\
\hline 4471.057 & 7 & 22359.80 & $22559^{\circ}{ }_{11 / 2}-44919_{9 / 2}$ & & & & 4421.357 & 1 & 22611.14 & $19132_{3 / 2}-41743_{3 / 2}^{\circ}$ & & & & \\
\hline 4470.985 & 2 & 22360.16 & & & & & 4420.845 & 40 & 22613.76 & $19753_{7 / 2}-42367_{5 / 2}^{\circ / 2}$ & So & 1.13 & 0.05 & \\
\hline 4470.425 & 4 & 22362.96 & & & & & 4420.236 & 7 & 22616.88 & $19753_{7 / 2}-42370^{\circ}{ }_{7 / 2}$ & $\Delta \mathrm{J}=0$ & 1.07 & 0.495 & \\
\hline 4470.360 & 2 & 22363.29 & & & & & 4419.787 & 2 & 22619.17 & & & & & \\
\hline 4470.012 & 40 & 22365.03 & $16957_{7 / 2}-39322_{9 / 2}^{\circ}$ & so & 1.03 & 0.12 & 4419.204 & 40 & 22622.16 & $19748_{9 / 2}-42370_{7 / 2}^{\circ}$ & SI & 2.14 & 0.86 & \\
\hline 4468.910 & 10 & 22370.54 & $19748_{9 / 2}-42119^{\circ}{ }_{11 / 2}$ & & & & 4419.046 & 20 & 22622.97 & $22468^{\circ}{ }_{11 / 2}-45091_{9 / 2}$ & & & & \\
\hline 4467.983 & 100 & 22375.19 & $15271_{15 / 2}-37646_{13 / 2}^{\circ}$ & so & 0.40 & 0.66 & 4417.470 & 20 & 22631.04 & $19548_{5 / 2}-42179_{5 / 2}^{\circ / 2}$ & $\Delta \mathrm{J}=0$ & 1.02 & 0.175 & \\
\hline 4467.694 & 7 & 22376.63 & $20406^{\circ}{ }_{5 / 2}-42783_{7 / 2}$ & & & & 4416.603 & $5 h$ & 22635.48 & & & & & \\
\hline 44.66 .643 & 2 & 22381.90 & $23781_{9 / 2}-46163_{11 / 2}^{\circ}$ & & & & 4416.240 & 2 & 22637.34 & & & & & \\
\hline 4466.078 & 70 & 22384.73 & $19748_{9 / 2}-42133^{\circ}{ }_{7 / 2}$ & SI & 1.71 & 0.425 & 4414.801 & 10 & 22644.72 & $16742_{7 / 2}-39386_{5 / 2}^{\circ}$ & & & & \\
\hline 4465.542 & 2 & 22387.42 & & & & & 4413.522 & 10 & 22651.28 & & SO & 0.35 & 0.815 & \\
\hline 4465.337 & 1 & 22388.44 & & & & & 4411.697 & 200 & 22660.65 & $8771_{5 / 2}^{\circ}-31431_{5 / 2}$ & $\Delta \mathrm{J}=0$ & 0.855 & 1.07 & \\
\hline 4464.778 & 1 & 22391.25 & & & & & 4410.842 & 5 & 22665.04 & & & & & \\
\hline
\end{tabular}


APPENDIX - Spectral Lines of Neutral Thulium-Continued

\begin{tabular}{|c|c|c|c|c|c|c|c|c|c|c|c|c|c|c|c|}
\hline \multirow{2}{*}{$\begin{array}{c}\text { Wave- } \\
\text { length } \\
\text { in air } \\
\AA\end{array}$} & \multirow[t]{2}{*}{ Intensity } & \multirow{2}{*}{$\begin{array}{c}\text { Wave- } \\
\text { number } \\
\mathrm{cm}^{-1}\end{array}$} & \multirow[t]{2}{*}{ Classification } & \multirow{2}{*}{\multicolumn{4}{|c|}{ Zeeman effect }} & $\begin{array}{l}\text { Wave- } \\
\text { length }\end{array}$ & Intensity & $\begin{array}{l}\text { Wave- } \\
\text { number }\end{array}$ & Classification & & eeman & ffect & \\
\hline & & & & & & & & $\begin{array}{c}\text { in air } \\
\AA\end{array}$ & & $\mathrm{cm}^{-1}$ & & type & f & $\mathrm{Je}$ & \\
\hline 4409.598 & 50 & 22671.44 & $16742_{7 / 2}-39413^{\circ}{ }_{7 / 2}$ & $\Delta \mathrm{J}=0$ & 1.14 & 0.595 & & 4355.695 & 60 & 22952.00 & $19753_{7 / 2}-42705_{s / 2}^{\circ}$ & SI & 1.40 & 0.22 & \\
\hline 4409.354 & 5 & 22672.69 & & & & & & 4354.917 & 30 & 22956.10 & & So & 0.22 & 0.81 & \\
\hline 4408.601 & 5 & 22676.56 & $22791_{7 / 2}-45467^{\circ}{ }_{9 / 2}$ & & & & & 4352.710 & $2 h$ & 22967.74 & & & & & \\
\hline 4407.560 & $3 h$ & 22681.92 & & & & & & 4352.028 & 80 & 22971.34 & $22742^{\circ}{ }_{13 / 2}-45714_{11 / 2}$ & & & & \\
\hline 4406.981 & 1 & 22684.90 & & & & & & 4351.375 & 3 & 22974.78 & & & & & \\
\hline 4404.476 & 1 & 22697.80 & & & & & & 4350.125 & 5 & 22981.39 & $19753_{7 / 2}-42735_{s / 2}^{\circ}$ & & & & \\
\hline 4403.650 & 40 & 22702.06 & $16742_{\tau_{/ 2}}-39444_{9 / 2}^{\circ}$ & so & 0.42 & 0.70 & & 4349.887 & 4 & 22982.64 & $17613_{9 / 2}^{/ 2}-40596_{7 / 2}^{\circ}$ & & & & \\
\hline 4402.325 & 2 & 22708.89 & $17613_{9 / 2}-40322_{9 / 2}^{\circ}$ & $\Delta \mathrm{J}=0$ & $?$ & 0.30 & & 4349.075 & 1 & 22986.93 & & & & & \\
\hline 4401.843 & 1 & 22711.38 & & & & & & 4348.841 & 20 & 22988.17 & $19753_{7 / 2}-42742^{\circ}{ }_{\tau_{2}}$ & $\Delta \mathrm{J}=0$ & 1.19 & 0.07 & \\
\hline 4401.082 & 2 & 22715.31 & $18853_{11 / 2}-41569^{\circ}{ }_{11 / 2}$ & $\Delta \mathrm{J}=0$ & $?$ & 0.275 & & 4347.840 & 50 & 22993.46 & $19748_{9 / 2}-42742^{\circ}{ }_{7 / 2}$ & SI & 1.62 & 0.33 & \\
\hline 4400.257 & 10 & 22718.56 & $21799^{\circ}{ }_{3 / 2}-44518_{5 / 2}$ & & & & & 4346.484 & 300 & 23000.64 & $15587_{11 / 2}-38588^{\circ}{ }_{13 / 2}$ & So & 1.00 & 0.205 & \\
\hline 4400.446 & 1 & 22718.59 & $21737_{9 / 2}^{1 / 2}-44456^{\circ}{ }_{7 / 2}$ & & & & & 4345.863 & 200 & 23003.92 & $17343_{7 / 2}-40347_{5 / 2}^{\circ}$ & So & 0.76 & 0.25 & \\
\hline 4400.070 & 20 & 22720.53 & $16742_{7 / 2}-39462^{\circ}{ }_{7 / 2}^{\circ}$ & & & & & 4345.129 & 3 & 23007.81 & & & & & \\
\hline 4399.723 & 100 & 22722.29 & $18853_{11 / 2}^{1 / 2}-41576_{9 / 2}^{\circ / 2}$ & so & 0.575 & 0.46 & & 4344.840 & 10 & 23009.34 & $21737_{9 / 2}-44747^{\circ}{ }_{11 / 2}$ & & & & \\
\hline 4398.573 & 40 & 22728.26 & $16742_{7 / 2}-39470^{\circ}{ }_{s / 2}$ & & & & & 4344.479 & 500 & 23011.25 & $17752_{5 / 2}-40763_{7 / 2}^{\circ}$ & $\mathrm{T}$ & 1.18 & & \\
\hline 4397.900 & 5 & 22731.74 & $18837_{9 / 2}-41569^{\circ}{ }_{11 / 2}$ & & & & & 4343.710 & 3 & 23015.32 & & & & & \\
\hline 4396.685 & 60 & 22738.02 & $16742_{7 / 2}-39480^{\circ}{ }_{7 / 2}$ & & & & & 4343.405 & 15 & 23016.94 & $19753_{7 / 2}-42770_{9 / 2}^{\circ}$ & SI & 1.30 & 0.11 & \\
\hline 4396.495 & $500 r$ & 22739.00 & $8771_{s / 2}^{\circ / 2}-31510_{7 / 2}^{/ 2}$ & SI & 0.856 & 1.11 & * & 4343.040 & 2 & 23018.88 & & & & & \\
\hline 4395.960 & 500 & 22741.77 & $15271_{15 / 2}-38012^{\circ}{ }_{13 / 2}$ & $\mathrm{~T}$ & 1.08 & 0.05 & & 4342.726 & 4 & 23020.54 & $18853_{11 / 2}-41874_{11 / 2}^{\circ}$ & & & & \\
\hline 4394.420 & $1000 r$ & 22749.74 & $8771_{s / 2}^{\circ}-31520_{3 / 2}$ & SI & 0.94 & 0.09 & & 4342.404 & 50 & 23022.25 & $19748_{9 / 2}-42770_{9 / 2}^{\circ}$ & $\Delta \mathrm{J}=0$ & 1.25 & 0.35 & \\
\hline 4393.309 & 4 & 22755.49 & & & & & & 4341.336 & 60 & 23027.91 & $18853_{11 / 2}-41881_{9 / 2}^{\circ}$ & so & 1.05 & 0.105 & \\
\hline 4392.435 & 2 & 22760.02 & $15587_{11 / 2}-38347^{\circ}{ }_{11 / 2}$ & & & & & 4339.626 & 30 & 23036.98 & $18837_{9 / 2}-41874_{11 / 2}^{\circ}$ & SI & 1.26 & 0.11 & \\
\hline 4392.220 & 4 & 22761.14 & & & & & & 4338.242 & 4 & 23044.33 & $18837_{9 / 2}-41881_{9 / 2}^{\circ / 2}$ & & & & \\
\hline 4390.670 & $2 h$ & 22769.17 & & & & & & 4337.622 & 70 & 23047.63 & $19132_{3 / 2}-42179^{\circ}{ }_{5 / 2}^{\circ}$ & SI & 0.88 & 1.06 & * \\
\hline 4389.952 & 30 & 22772.90 & & SI & 1.51 & 0.32 & & 4336.554 & 3 & 23053.30 & & & & & \\
\hline 4389.551 & 100 & 22774.98 & & so & 0.99 & 0.215 & & 4334.656 & 30 & 23063.40 & $18853_{11 / 2}-41917^{\circ}{ }_{13 / 2}$ & $\mathrm{~T}$ & 1.16 & & \\
\hline 4389.207 & 2 & 22776.76 & $21997_{11 / 2}-44774_{9 / 2}^{\circ}$ & & & & & 4333.737 & 3 & 23068.29 & & & & & \\
\hline 4388.355 & 40 & 22781.18 & $17752_{s / 2}-40533^{\circ}{ }_{7 / 2}$ & SI & 1.37 & 0.145 & & 4332.589 & $20 h$ & 23074.40 & $20406_{s / 2}^{\circ}-43481_{s / 2}$ & & & & \\
\hline 4387.641 & $10 h$ & 22784.89 & $16957_{7 / 2}-39741_{9 / 2}^{\circ}$ & & & & & 4330.851 & 1 & 23083.66 & & & & & \\
\hline 4387.360 & 5 & 22786.35 & & & & & & 4330.476 & 7 & 23085.66 & $19753_{7 / 2}-42839_{9 / 2}^{\circ}$ & so & 0.72 & 0.35 & \\
\hline 4386.434 & $10000 a$ & 22791.16 & $0_{7 / 2}^{\circ}-22791_{7 / 2}$ & $\mathrm{~T}$ & 1.14 & & & 4329.487 & 8 & 23090.93 & $19748_{9 / 2}-42839_{9 / 2}^{\circ}$ & $\Delta \mathrm{J}=0$ & 1.29 & 1.09 & * \\
\hline 4386.120 & 10 & 22792.79 & $19748_{9 / 2}-42541^{\circ}{ }_{11 / 2}$ & & & & & 4328.190 & 20 & 23097.85 & & SI & 1.26 & 0.04 & \\
\hline 4385.817 & $4 h$ & 22794.36 & & & & & & 4327.308 & 2 & 23102.56 & $21161_{5 / 2}-44263^{\circ}{ }_{7 / 2}$ & & & & \\
\hline 4385.535 & 30 & 22795.83 & $18990_{11 / 2}-41786_{9 / 2}^{\circ}$ & so & 1.09 & 0.13 & & 4326.987 & 6 & 23104.27 & $16957_{7 / 2}-40061_{7 / 2}^{\circ}$ & $\Delta \mathrm{J}=0$ & 1.14 & 0.13 & \\
\hline 4385.291 & 20 & 22797.10 & $15587_{11 / 2}-38384_{9 / 2}^{\circ}$ & & & & & 4325.688 & $6 h$ & 23111.21 & & & & & \\
\hline 4385.095 & 4 & 22798.12 & & & & & & 4324.116 & 10 & 23119.61 & & so & 0.76 & 0.32 & \\
\hline 4384.743 & $100 h$ & 22799.95 & $16742_{7 / 2}-39542^{\circ}{ }_{7 / 2}$ & & & & & 4322.423 & 20 & 23128.67 & $18990_{11 / 2}-42119^{\circ}{ }_{11 / 2}$ & $\Delta \mathrm{J}=0$ & 1.15 & 0.69 & \\
\hline 4383.510 & 5 & 22806.36 & $21997_{11 / 2}-44803^{\circ}{ }_{13 / 2}$ & & & & & 4320.522 & 2 & 23138.84 & $2116 l_{5 / 2}-44300_{5 / 2}^{\circ}$ & & & & \\
\hline 4383.257 & 6 & 22807.68 & $19753_{7 / 2}-42561_{9 / 2}^{\circ}$ & & & & & 4319.726 & 3 & 23143.11 & $21120_{7 / 2}-44263^{\circ}{ }_{7 / 2}^{\circ}$ & & & & \\
\hline 4382.432 & $6 h$ & 22811.97 & $22902^{\circ}{ }_{13 / 2}^{\circ}-45714_{11 / 2}$ & & & & & 4318.397 & $600 r$ & 23150.23 & $17613_{9 / 2}-40763^{\circ}{ }_{7 / 2}$ & SI & 1.25 & 0.05 & \\
\hline 4382.243 & $3 h$ & 22812.96 & $19748_{9 / 2}-42561_{9 / 2}^{\circ}$ & & & & & 4317.126 & 20 & 23157.05 & $19548_{5 / 2}-42705_{5 / 2}^{\circ}$ & $\Delta \mathrm{J}=0$ & 1.05 & 0.30 & \\
\hline 4380.360 & 5 & 22822.76 & & & & & & 4316.215 & 2 & 23161.93 & & & & & \\
\hline 4379.989 & $20 h$ & 22824.69 & & & & & & 4315.642 & $30 h$ & 23165.01 & & & & & \\
\hline 4378.310 & 100 & 22833.45 & $19748_{9 / 2}-42581_{11 / 2}^{\circ}$ & so & 0.46 & 0.675 & & 4314.955 & 2 & 23168.70 & & & & & \\
\hline 4377.017 & 50 & 22840.19 & $19753_{7 / 2}-42594_{9 / 2}^{\circ}$ & So & 0.68 & 0.36 & & 4313.916 & 3 & 23174.28 & $17343_{7 / 2}-40517_{9 / 2}^{\circ}$ & & & & \\
\hline 4376.475 & 4 & 22843.02 & & & & & & 4312.966 & 8 & 23179.38 & $21120_{\gamma^{2} / 2}-44300^{\circ}{ }_{5 / 2}$ & SI & 1.33 & 0.16 & \\
\hline 4376.344 & 8 & 22843.70 & $17752_{s / 2}-40596_{7 / 2}^{\circ}$ & & & & & 4312.084 & 3 & 23184.12 & & & & & \\
\hline 4376.003 & 50 & 22845.48 & $19748_{9 / 2}-42594_{9 / 2}^{\circ}$ & $\Delta \mathrm{J}=0$ & 1.19 & 0.875 & & 4311.743 & 2 & 23185.96 & $17752_{5 / 2}-40938_{s / 2}^{\circ}$ & & & & \\
\hline 4375.766 & 10 & 22846.72 & $23374_{13 / 2}^{1 / 2}-46221_{11 / 2}^{\circ}$ & & & & & 4311.672 & 2 & 23186.34 & $19548_{5 / 2}-42735_{5 / 2}^{\circ}$ & & & & \\
\hline 4375.140 & 100 & 22849.99 & $18837_{9 / 2}-41687^{\circ}{ }_{11 / 2}^{1 / 2}$ & SI & 1.24 & 0.10 & & 4311.543 & 2 & 23187.03 & $18693_{15 / 2}^{/ 2}-41880^{\circ}{ }_{15 / 2}^{\circ / 2}$ & & & & \\
\hline 4373.053 & 10 & 22860.90 & & so & 0.35 & 0.585 & & 4310.405 & 30 & 23193.15 & $19548_{5 / 2}-42742^{\circ}{ }_{\tau / 2}$ & SI & 0.98 & 1.19 & * \\
\hline 4372.882 & $10 h$ & 22861.79 & & & & & & 4309.819 & 2 & 23196.31 & & & & & \\
\hline 4372.247 & 3 & 22865.11 & $17343_{7 / 2}-40208_{9 / 2}^{\circ}$ & & & & & 4309.623 & 3 & 23197.36 & & & & & \\
\hline 4369.948 & 4 & 22877.14 & & & & & & 4307.593 & $2 h$ & 23208.29 & $17454_{13 / 2}-40663^{\circ}{ }_{11 / 2}$ & & & & \\
\hline 4369.557 & $3 h$ & 22879.19 & & & & & & 4306.780 & 10 & 23212.67 & & & & & \\
\hline 4368.648 & 50 & 22883.95 & $18990_{11 / 2}-41874_{11 / 2}^{\circ}$ & $\Delta \mathrm{J}=0$ & 1.19 & 0.32 & & 4306.657 & 150 & 23213.34 & $19748_{9 / 2}-42961_{11 / 2}^{\circ}$ & so & 0.83 & 0.385 & \\
\hline 4367.238 & 200 & 22891.33 & $18990_{11 / 2}-41881^{\circ}{ }^{\circ / 2}$ & SI & 1.44 & 0.225 & & 4306.378 & 40 & 23214.84 & $15271_{15 / 2}-38485^{\circ}{ }_{15 / 2}$ & $\Delta \mathrm{J}=0$ & $?$ & 0.472 & \\
\hline 4366.295 & $50 h$ & 22896.28 & $20406^{\circ}{ }_{5 / 2}^{\circ / 2}-43303_{7 / 2}^{7 / 2}$ & & & & & 4305.490 & 4 & 23219.63 & & & & & \\
\hline 4365.936 & 3 & 22898.16 & & & & & & 4304.865 & 2 & 23223.00 & $23374_{13 / 2}-46597^{\circ}{ }_{13 / 2}$ & & & & \\
\hline 4365.294 & 3 & 22901.53 & & & & & & 4304.652 & 5 & 23224.15 & $18693_{15 / 2}-41917^{\circ}{ }_{13 / 2}$ & & & & \\
\hline 4364.898 & $10 h$ & 22903.61 & & & & & & 4302.569 & 20 & 23235.39 & $19132_{3 / 2}-42367_{5 / 2}^{\circ}$ & SI & 0.88 & 1.20 & \\
\hline 4364.820 & 3 & 22904.02 & $17613_{9 / 2}-40517_{9 / 2}^{\circ}$ & $\Delta \mathrm{J}=0$ & 1.17 & 0.13 & & 4302.023 & 3 & 23238.34 & & & & & \\
\hline 4364.113 & 3 & 22907.73 & & & & & & 4301.360 & 20 & 23241.92 & $17613_{9 / 2}-40855_{9 / 2}^{\circ}$ & $\Delta \mathrm{J}=0$ & 1.14 & 0.475 & \\
\hline 4363.455 & 40 & 22911.18 & $15587_{11 / 2}-38499_{9 / 2}^{\circ}$ & SI & 1.25 & 1.07 & * & 4300.595 & 20 & 23246.06 & $22468^{\circ}{ }_{11 / 2}-45714_{11 / 2}$ & & & & \\
\hline 4362.422 & $1 h$ & 22916.61 & $16742_{7 / 2}-39658_{5 / 2}^{\circ}$ & & & & & 4299.589 & 20 & 23251.50 & $16957_{7^{2} / 2}-40208_{9 / 2}^{\circ}$ & SI & 1.24 & 0.04 & \\
\hline 4361.746 & 10 & 22920.16 & $17613_{9 / 2}^{/ 2}-40533_{7 / 2}^{\circ}$ & & & & & 4299.482 & 4 & 23252.08 & & & & & \\
\hline 4360.476 & 15 & 22926.83 & $18990_{11 / 2}-4191^{7}{ }^{7 / 2}$ & & & & & 4299.325 & 7 & 23252.92 & $17343_{\tau / 2}-40596_{\tau_{/ 2}}^{\circ}$ & & & & \\
\hline 4359.928 & $15000 a$ & 22929.71 & $0_{7 / 2}^{\circ} \quad 22929_{5 / 2}$ & $\mathrm{~T}$ & 1.15 & & & 4298.365 & $300 r$ & 23258.12 & $13119_{9 / 2}-36377_{9 / 2}^{\circ}$ & $\Delta \mathrm{J}=0$ & 1.27 & 0.46 & \\
\hline 4357.975 & 6 & 22939.99 & $19466_{13 / 2}-42406^{\circ}{ }_{13 / 2}$ & & & & & 4298.060 & 40 & 23259.77 & $17752_{5 / 2}-41012_{7 / 2}^{\circ}$ & so & 1.03 & 0.11 & \\
\hline 4357.620 & 4 & 22941.86 & $15587_{11 / 2}-38529^{\circ}{ }_{11 / 2}$ & & & & & 4297.477 & 2 & 23262.92 & & & & & \\
\hline
\end{tabular}


APPENDIX - Spectral Lines of Neutral Thulium-Continued

\begin{tabular}{|c|c|c|c|c|c|c|c|c|c|c|c|c|c|c|c|}
\hline $\begin{array}{c}\text { Wave- } \\
\text { length } \\
\text { in air } \\
\AA\end{array}$ & Intensity & $\begin{array}{c}\text { Wave- } \\
\text { number } \\
\mathrm{cm}^{-1}\end{array}$ & Classification & \multicolumn{4}{|c|}{$\begin{array}{l}\text { Zeeman effect } \\
\text { e f f Je }\end{array}$} & $\begin{array}{c}\text { Wave- } \\
\text { length } \\
\text { in air } \\
\AA\end{array}$ & Intensity & $\begin{array}{l}\text { Wave- } \\
\text { number } \\
\mathrm{cm}^{-1}\end{array}$ & Classification & type $^{\mathrm{Ze}}$ & $\underset{f}{\text { Leeman }} \mathrm{e}$ & Jeffect & \\
\hline 4297.044 & 20 & 23265.27 & $18853_{11 / 2}-42119^{\circ}{ }_{11 / 2}$ & $\Delta \mathrm{J}=0$ & 1.13 & 0.32 & & 4237.235 & $10 h$ & 23593.65 & & & & & \\
\hline 4296.052 & $4 h$ & 23270.64 & & & & & & 4236.400 & $80 h$ & 23598.30 & $17613_{9 / 2}-41211_{7 / 2}^{\circ}$ & & & & \\
\hline 4294.982 & 20 & 23276.44 & $19753_{\tau_{/ 2}}-43030_{9 / 2}^{\circ}$ & SI & $?$ & 0.325 & & 4236.237 & $3 h$ & 23599.21 & & & & & \\
\hline 4294.481 & 2 & 23279.15 & & & & & & 4235.444 & 40 & 23603.63 & $18990_{11 / 2}-42594_{9 / 2}^{\circ}$ & SI & 1.70 & 0.48 & \\
\hline 4293.289 & 30 & 23285.62 & $19748_{9 / 2}-43034^{\circ}{ }_{7 / 2}$ & SI & 1.77 & 0.445 & & 4235.192 & 40 & 23605.03 & $16742_{7 / 2}-40347^{\circ}{ }_{5 / 2}$ & SI & 1.325 & 1.135 & * \\
\hline 4292.591 & 3 & 23289.40 & $15587_{11 / 2}-38877^{\circ}{ }_{11 / 2}$ & & & & & 4235.036 & 3 & 23605.90 & & & & & \\
\hline 4291.590 & $4 h$ & 23294.83 & $21161_{5 / 2}-44456_{7 / 2}^{\circ}$ & & & & & 4234.177 & 4 & 23610.69 & & & & & \\
\hline 4291.084 & 2 & 23297.58 & $21120_{7 / 2}-44418^{\circ}{ }_{7 / 2}^{\circ / 2}$ & & & & & 4232.395 & 2 & 23620.63 & & & & & \\
\hline 4290.874 & 60 & 23298.72 & $19753_{\tau_{/ 2}}-43052^{\circ}{ }_{\tau_{/ 2}}$ & $\Delta \mathrm{J}=0$ & 1.12 & 0.46 & & 4232.116 & 4 & 23622.19 & $24137_{13 / 2}-47759^{\circ}{ }_{13 / 2}$ & & & & \\
\hline 4290.137 & $20 h$ & 23302.72 & & & & & & 4231.332 & 4 & 23626.57 & $17454_{13 / 2}-41081_{11 / 2}^{\circ}$ & & & & \\
\hline 4289.905 & 20 & 23303.98 & $19748_{9 / 2}-43052^{\circ}{ }_{7 / 2}$ & SI & 1.29 & 1.055 & * & 4229.236 & 2 & 23638.28 & $21997_{11 / 2}-45635^{\circ}{ }_{11 / 2}$ & & & & \\
\hline 4288.653 & 20 & 23310.79 & & SI & 1.17 & 0.215 & & 4229.058 & 40 & 23639.27 & $16957_{7 / 2}-40596^{\circ}{ }_{7 / 2}$ & $\Delta \mathrm{J}=0$ & 1.23 & 0.47 & \\
\hline 4287.426 & 10 & 23317.46 & $15271_{15 / 2}-38588^{\circ}{ }_{13 / 2}$ & & & & & 4228.050 & 40 & 23644.91 & $15271_{15 / 2}-38915^{\circ}{ }_{13 / 2}^{1 / 2}$ & & & & \\
\hline 4287.130 & $2 h$ & 23319.07 & $16742_{7 / 2}-40061^{\circ}{ }_{7 / 2}$ & & & & & 4227.390 & 2 & 23648.60 & & & & & \\
\hline 4286.934 & $40 h$ & 23320.13 & & & & & & 4226.537 & 2 & 23653.37 & $21120_{7^{\prime} / 2}-44774_{9 / 2}^{\circ}$ & & & & \\
\hline 4286.102 & 2 & 23324.66 & & & & & & 4226.253 & 3 & 23654.96 & & & & & \\
\hline 4285.840 & 20 & 23326.09 & $17454_{13 / 2}-40780^{\circ}{ }_{13 / 2}$ & $\Delta \mathrm{J}=0$ & 1.18 & 0.465 & & 4226.055 & 150 & 23656.07 & & SI & 1.20 & 0.11 & \\
\hline 4285.654 & 3 & 23327.10 & & & & & & 4225.639 & 10 & 23658.40 & & & & & \\
\hline 4285.073 & 4 & 23330.26 & & & & & & 4225.565 & 4 & 23658.81 & & & & & \\
\hline 4284.680 & 40 & 23332.40 & $17752_{5 / 2}-41085^{\circ}{ }_{7 / 2}$ & & & & & 4223.060 & 20 & 23672.84 & $17752_{5 / 2}-41425^{\circ}{ }_{7 / 2}$ & & & & \\
\hline 4284.344 & 4 & 23334.23 & & & & & & 4222.671 & $2000 r$ & 23675.03 & $8771_{\mathrm{s} / 2}^{\circ}-32446_{7 / 2}$ & SI & 1.00 & 0.10 & \\
\hline 4284.095 & 3 & 23335.59 & & & & & & 4222.584 & 200 & 23675.51 & $19753_{7 / 2}-43429_{9 / 2}^{\circ}$ & & & & \\
\hline 4281.662 & 2 & 23348.85 & & & & & & 4222.336 & 8 & 23676.90 & $19753_{7 / 2}-43430^{\circ}{ }_{5 / 2}$ & & & & \\
\hline 4280.776 & 10 & 23353.68 & $19548_{5 / 2}-42902_{s / 2}^{\circ}$ & & & & & 4221.743 & 10 & 23680.23 & $19748_{9 / 2}-43428^{\circ}{ }_{7 / 2}$ & & & & \\
\hline 4280.369 & 3 & 23355.90 & & & & & & 4221.639 & 80 & 23680.81 & $19748_{9 / 2}^{/ 2}-43429^{\circ}{ }_{9 / 2}^{/ 2}$ & $\Delta \mathrm{J}=0$ & 1.25 & 0.47 & \\
\hline 4279.990 & 2 & 23357.97 & & & & & & 4221.450 & 50 & 23681.87 & $19548_{5 / 2}-43230_{3 / 2}^{\circ}$ & $\mathrm{T}$ & 1.00 & 0.04 & \\
\hline 4279.620 & $10 h$ & 23359.99 & $22902^{\circ}{ }_{13 / 2}-46262_{11 / 2}$ & & & & & 4220.440 & 3 & 23687.54 & $18853_{11 / 2}-42541^{\circ}{ }_{11 / 2}$ & & & & \\
\hline 4278.605 & 3 & 23365.53 & $16957_{7 / 2}-40322_{9 / 2}^{\circ}$ & & & & & 4218.060 & 4 & 23700.91 & & & & & \\
\hline 4274.355 & 60 & 23388.76 & $19466_{13 / 2}-42855^{\circ}{ }_{15 / 2}$ & so & 0.93 & 0.185 & & 4217.753 & $20 h$ & 23702.63 & $22559^{\circ}{ }_{11 / 2}-46262_{11 / 2}$ & $\mathrm{~T}$ & 1.16 & & \\
\hline 4274.084 & 7 & 23390.24 & $16957_{7 / 2}-40347^{\circ}{ }^{\circ}$ & & & & & 4217.030 & $3 h$ & 23706.69 & & & & & \\
\hline 4273.839 & 4 & 23391.58 & $21997_{11 / 2}-45389^{\circ}{ }_{13 / 2}$ & & & & & 4216.851 & 60 & 23707.70 & $18853_{11 / 2}-42561_{9 / 2}^{\circ}$ & SI & 1.69 & 0.55 & \\
\hline 4272.537 & 60 & 23398.71 & $17613_{9 / 2}-41012^{\circ}{ }^{\circ}$ & SI & 1.32 & 0.235 & & 4215.804 & 20 & 23713.59 & $18693_{15 / 2}-42406^{\circ / 2 / 2}$ & & & & \\
\hline 4271.716 & $500 r$ & 23403.21 & $8771_{s / 2}^{\circ / 2}-32174_{s / 2}$ & $\Delta \mathrm{J}=0$ & 0.856 & 1.16 & * & 4215.627 & $10 h$ & 23714.58 & & & & & \\
\hline 4270.190 & $2 h$ & 23411.57 & & & & & & 4213.925 & 10 & 23724.16 & $18837_{9 / 2}-42561_{9 / 2}^{\circ}$ & $\Delta \mathrm{J}=0$ & 1.70 & 0.36 & \\
\hline 4269.334 & 3 & 23416.27 & $18990_{11 / 2}-42406^{\circ}{ }_{13 / 2}$ & & & & & 4213.216 & 200 & 23728.15 & $18853_{11 / 2}-42581^{\circ}{ }_{11 / 2}$ & $\mathrm{~T}$ & 1.14 & & \\
\hline 4268.561 & 300 & 23420.51 & $17343_{7 / 2}-40763^{\circ}{ }_{7 / 2}$ & $\Delta \mathrm{J}=0$ & 1.02 & 1.18 & * & 4212.588 & 10 & 23731.69 & & & & & \\
\hline 4265.648 & $20 h$ & 23436.50 & $17752_{5 / 2}-41189^{\circ}{ }_{7 / 2}^{\circ / 2}$ & & & & & 4211.980 & $20 h$ & 23735.12 & $17343_{7 / 2}-41078_{9 / 2}^{\circ}$ & SI & 1.02 & 1.32 & * \\
\hline 4265.414 & 30 & 23437.79 & $19748_{9 / 2}-43186^{\circ}{ }_{11 / 2}$ & & & & & 4211.720 & 4 & 23736.58 & & & & & \\
\hline 4264.394 & 2 & 23443.39 & $19466_{13 / 2}-42910^{\circ}{ }_{13 / 2}$ & & & & & 4211.332 & 2 & 23738.77 & & & & & \\
\hline 4262.798 & $3 h$ & 23452.17 & $20406_{s / 2}^{\circ}-43859_{s / 2}$ & & & & & 4211.072 & 6 & 23740.23 & $18853_{11 / 2}-42594_{9 / 2}^{\circ}$ & & & & \\
\hline 4262.486 & $10 h$ & 23453.89 & & & & & & 4210.921 & 7 & 23741.09 & & & & & \\
\hline 4261.502 & $60 h$ & 23459.30 & $17752_{s / 2}-41211^{\circ}{ }_{7 / 2}$ & & & & & 4210.817 & 40 & 23741.67 & $17343_{7 / 2}-41085^{\circ}{ }_{7 / 2}$ & $\Delta \mathrm{J}=0$ & 1.015 & 0.08 & \\
\hline 4261.200 & 2 & 23460.96 & & & & & & 4210.299 & 6 & 23744.59 & $18837_{9 / 2}-42581^{\circ}{ }_{11 / 2}^{1 / 2}$ & & & & \\
\hline 4260.487 & $300 h$ & 23464.89 & $17613_{9 / 2}-41078_{9 / 2}^{\circ}$ & $\Delta \mathrm{J}=0$ & 1.27 & 0.63 & & 4209.811 & 5 & 23747.35 & $21120_{7 / 2}-44868_{9 / 2}^{\circ / 2}$ & & & & \\
\hline 4259.975 & 3 & 23467.71 & $17613_{9 / 2}-41081^{\circ}{ }_{11 / 2}$ & & & & & 4208.144 & $3 h$ & 23756.75 & $18837_{9 / 2}-42594_{9 / 2}^{\circ}$ & $\mathrm{T}$ & 1.125 & & \\
\hline 4259.307 & 10 & 23471.39 & $17613_{9 / 2}-41085^{\circ}{ }_{7 / 2}^{1 / 2}$ & SI & 1.86 & 0.65 & & 4207.832 & 3 & 23758.51 & & & & & \\
\hline 4255.283 & 15 & 23493.59 & & $\Delta \mathrm{J}=0$ & 1.14 & 0.44 & & 4207.322 & 6 & 23761.39 & & SI & 1.60 & 0.37 & \\
\hline 4254.988 & 20 & 23495.22 & $19466_{13 / 2}-42961^{\circ}{ }_{11 / 2}$ & so & 0.89 & 0.25 & & 4207.268 & $4 h$ & 23761.70 & & & & & \\
\hline 4254.905 & 4 & 23495.67 & $17613_{9 / 2}-41109_{9 / 2}^{\circ}$ & & & & & 4206.508 & 3 & 23765.99 & $17343_{7 / 2}-41109_{9 / 2}^{\circ}$ & & & & \\
\hline 4254.335 & 6 & 23498.82 & & & & & & 4205.752 & 40 & 23770.26 & $19132_{3 / 2}-42902^{\circ}{ }_{5 / 2}$ & SI & 0.88 & 1.04 & * \\
\hline 4253.455 & 200 & 23503.68 & $19548_{s / 2}-43052^{\circ}{ }_{\tau / 2}$ & SI & 1.24 & 0.18 & & 4204.841 & 5 & 23775.4 .1 & $16742_{7 / 2}-40517_{9 / 2}^{\circ}$ & & & & \\
\hline 4252.191 & 30 & 23510.67 & $17613_{9 / 2}-41124_{9 / 2}^{\circ}$ & $\Delta \mathrm{J}=0$ & 1.22 & 0.26 & & 4203.953 & 30 & 23780.44 & $18990_{11 / 2}-42770^{\circ}{ }_{9 / 2}$ & $\mathrm{~T}$ & 1.22 & & \\
\hline 4251.362 & 10 & 23515.25 & & $\Delta \mathrm{J}=0$ & 1.02 & 1.02 & & 4203.727 & $30000 a$ & 23781.71 & $0_{\tau / 2}^{\circ}-23781_{8 / 2}$ & $\mathrm{~T}$ & 1.14 & & \\
\hline 4250.624 & 30 & 23519.34 & $22742^{\circ}{ }_{13 / 2}-46262_{11 / 2}$ & & & & & 4201.980 & 3 & 23791.60 & $16742_{7 / 2}-40533^{\circ}{ }_{7 / 2}$ & & & & \\
\hline 4250.316 & 3 & 23521.04 & & & & & & 4201.542 & $10 h$ & 23794.08 & $22468^{\circ}{ }_{11 / 2}-46262_{11 / 2}$ & & & & \\
\hline 4247.416 & 150 & 23537.10 & $13119_{9 / 2}-36656^{\circ}{ }_{11 / 2}$ & so & 1.304 & 1.16 & * & 4201.195 & 4 & 23796.05 & $19748_{9 / 2}-43544_{9 / 2}^{\circ}$ & & & & \\
\hline 4244.919 & 30 & 23550.95 & $18990_{11 / 2}-42541_{11 / 2}^{\circ}$ & $\Delta \mathrm{J}=0$ & 1.15 & 0.64 & & 4200.577 & 100 & 23799.55 & $20406_{\mathrm{s} / 2}^{\circ / 2}-44206_{\tau / 2}$ & S00 & 1.58 & 1.04 & * \\
\hline 4244.838 & 5 & 23551.39 & $20406^{\circ}{ }_{5 / 2}^{\circ}-43958_{s / 2}$ & & & & & 4199.284 & 30 & 23806.88 & $16957_{7 / 2}^{/ / 2}-40763^{\circ / 2}{ }_{7 / 2}^{12}$ & $T$ & 1.17 & & \\
\hline 4244.576 & 4 & 23552.85 & $18853_{11 / 2}-42406^{\circ}{ }_{13 / 2}$ & & & & & 4198.411 & 100 & 23811.83 & $17613_{9 / 2}^{1 / 2}-41425_{7 / 2}^{\circ}$ & SI & 1.56 & 0.33 & \\
\hline 4243.325 & 10 & 23559.79 & & & & & & 4198.270 & $4 h$ & 23812.63 & & & & & \\
\hline 4243.170 & 20 & 23560.65 & $16957_{7 / 2}-40517_{9 / 2}^{\circ}$ & $\mathrm{T}$ & 1.16 & & & 4198.200 & 2 & 23813.02 & $21997_{11 / 2}-45810^{\circ}{ }_{11 / 2}$ & & & & \\
\hline 4242.420 & 30 & 23564.82 & $19548_{5 / 2}-43113^{\circ}{ }_{5 / 2}$ & $\Delta \mathrm{J}=0$ & $?$ & 0.325 & & 4197.924 & 20 & 23814.59 & $13119_{9 / 2}-36934^{\circ / 2}$ & SI & 1.40 & 0.09 & \\
\hline 4241.528 & $30 h$ & 23569.77 & $15587_{11 / 2}-39157^{\circ}{ }_{13 / 2}$ & & & & & 4196.958 & 5 & 23820.07 & $17454_{13 / 2}-41274_{13 / 2}^{\circ}$ & $\mathrm{T}$ & 1.14 & & \\
\hline 4241.282 & 40 & 23571.14 & $18990_{11 / 2}-42561_{9 / 2}^{\circ}$ & SI & 1.215 & 1.055 & * & 4196.608 & 10 & 23822.06 & $19466_{13 / 2}-43288^{\circ}{ }_{13 / 2}$ & $\Delta \mathrm{J}=0$ & 1.17 & 0.27 & \\
\hline 4240.722 & 20 & 23574.25 & $20406^{\circ}{ }_{5 / 2}-43981_{7 / 2}$ & & & & & 4196.419 & 4 & 23823.13 & & & & & \\
\hline 4240.490 & $200 h$ & 23575.54 & $17613_{9 / 2}-41189^{\circ}{ }_{7 / 2}$ & $\mathrm{~T}$ & 1.19 & & & 4194.848 & 4 & 23832.05 & & & & & \\
\hline 4239.632 & 6 & 23580.31 & $16742_{7 / 2}-40322_{9 / 2}^{\circ}$ & & & & & 4194.440 & 10 & 23834.37 & $19753_{7 / 2}-43588^{\circ}{ }_{7 / 2}$ & & & & \\
\hline 4239.012 & 1 & 23583.76 & & & & & & 4194.054 & 2 & 23836.56 & $20406^{\circ}{ }_{s / 2}^{2}-44243_{5 / 2}$ & & & & \\
\hline 4238.809 & 20 & 23584.89 & $19466_{13 / 2}-43051^{\circ}{ }_{15 / 2}$ & SI & 1.42 & 0.24 & & 4193.725 & 3 & 23838.43 & & & & & \\
\hline 4237.612 & 20 & 23591.55 & $18990_{11 / 2}-42581^{\circ}{ }_{11 / 2}^{/ 2}$ & $\Delta \mathrm{J}=0$ & 1.17 & 0.43 & & 4193.514 & 40 & 23839.63 & $19748_{9 / 2}-43588^{\circ}{ }_{\gamma / 2}$ & SI & 1.44 & 0.15 & \\
\hline
\end{tabular}


APPENDIX - Spectral Lines of Neutral Thulium - Continued

\begin{tabular}{|c|c|c|c|c|c|c|c|c|c|c|c|c|c|c|}
\hline \multirow{2}{*}{$\begin{array}{c}\text { Wave- } \\
\text { length } \\
\text { in air } \\
\AA\end{array}$} & \multirow[t]{2}{*}{ Intensity } & \multirow{2}{*}{$\begin{array}{l}\text { Wave- } \\
\text { number } \\
\mathrm{cm}^{-1}\end{array}$} & \multirow[t]{2}{*}{ Classification } & \multirow{2}{*}{\multicolumn{4}{|c|}{ Zeeman effect }} & \multirow{2}{*}{$\begin{array}{c}\text { Wave- } \\
\text { length } \\
\text { in air } \\
\AA\end{array}$} & Intensity & $\begin{array}{l}\text { W ave- } \\
\text { number }\end{array}$ & Classification & & eeman el & ffect \\
\hline & & & & & & & & & & & & type & f & $\mathrm{Je}$ \\
\hline 4193.028 & $10 h$ & 23842.40 & $22419_{9 / 2}^{\circ}-46262_{11 / 2}$ & & & & & 4138.334 & $2000 r$ & 24157.50 & $8771_{s / 2}^{\circ}-32928_{3 / 2}$ & so & 0.74 & 0.12 \\
\hline 4192.336 & 60 & 23846.33 & $15587_{11 / 2}-39434_{13 / 2}^{\circ}$ & So & 0.57 & 0.58 & & 4138.005 & $100 h$ & 24159.42 & & & & \\
\hline 4191.846 & 4 & 23849.12 & $18990_{11 / 2}-42839_{9 / 2}^{\circ}$ & & & & & 4137.503 & 70 & 24162.35 & $18693_{15 / 2}-42855^{\circ}{ }_{15 / 2}$ & $\Delta \mathrm{J}=0$ & 1.15 & 0.43 \\
\hline 4190.975 & 100 & 23854.07 & $16742_{\tau / 2}-40596^{\circ}{ }_{7 / 2}$ & $\Delta \mathrm{c}^{\top}=0$ & 1.315 & 0.07 & & 4137.365 & 4 & 24163.16 & $15271_{15 / 2}-39434_{13 / 2}^{\circ}$ & & & \\
\hline 4190.204 & 20 & 23858.46 & $20406^{\circ / 2}-44265_{7 / 2}^{1 / 2}$ & SOO & 1.58 & 1.10 & * & 4136.360 & $9 h$ & 24169.03 & & & & \\
\hline 4187.615 & $40000 a$ & 23873.21 & $0_{7 / 2}^{\circ}-23873_{7 / 2}^{\circ / 2}$ & $\Delta \mathrm{J}=0$ & 1.14 & 0.04 & & 4136.192 & $30 h$ & 24170.01 & & & & \\
\hline 4186.086 & 20 & 23881.93 & $19548_{s / 2}^{/ 2}-43430^{\circ}{ }_{s / 2}$ & & & & & 4135.875 & 9 & 24171.86 & & & & \\
\hline 4184.513 & 100 & 23890.91 & $13119_{9 / 2}-37010_{9 / 2}^{\circ}$ & $\Delta \mathrm{J}=0$ & 1.305 & 1.105 & * & 4135.744 & 4 & 24172.63 & $17613_{9 / 2}-41786_{9 / 2}^{\circ}$ & & & \\
\hline 4184.311 & 6 & 23892.06 & $15587_{11 / 2}-39479^{\circ}{ }_{11 / 2}$ & & & & & 4135.462 & $6 h$ & 24174.28 & & & & \\
\hline 4184.064 & $6 h$ & 23893.47 & $17752_{s / 2}-41646_{7 / 2}^{\circ}$ & & & & & 4135.083 & 2 & 24176.49 & $18853_{11 / 2}-43030_{9 / 2}^{\circ}$ & & & \\
\hline 4183.245 & $6 h$ & 23898.15 & & & & & & 4134.167 & 8 & 24181.85 & $17343_{7 / 2}-41525_{7 / 2}^{\circ}$ & $\Delta \mathrm{J}=0$ & 1.05 & 0.27 \\
\hline 4183.168 & 3 & 23898.59 & $16957_{7 / 2}-40855_{9 / 2}^{\circ}$ & & & & & 4134.068 & 1 & 24182.43 & & & & \\
\hline 4182.898 & 3 & 23900.13 & & & & & & 4132.740 & 6 & 24190.20 & $17343_{7_{/ 2}}-41533_{s / 2}^{\circ}$ & & & \\
\hline 4182.848 & 7 & 23900.42 & $15587_{11 / 2}-39488_{9 / 2}^{\circ}$ & & & & & 4132.270 & 5 & 24192.95 & $18837_{9 / 2}-43030_{9 / 2}^{\circ}$ & & & \\
\hline 4182.110 & 4 & 23904.64 & $18837_{9 / 2}-42742^{\circ}{ }_{7 / 2}$ & & & & & 4131.760 & 10 & 24195.94 & $18990_{11 / 2}-43186_{11 / 2}^{\circ}$ & & & \\
\hline 4180.902 & 40 & 23911.54 & $17613_{9 / 2}-41525_{7 / 2}^{\circ}$ & SI & 1.46 & 0.29 & & 4131.658 & $4 h$ & 24196.53 & & & & \\
\hline 4180.093 & 30 & 23916.17 & $20406_{s / 2}^{\circ}-44323_{7 / 2}$ & SOO & 1.58 & 1.09 & * & 4130.998 & 2 & 24200.40 & & & & \\
\hline 4179.953 & 50 & 23916.97 & $18853_{11 / 2}-42770_{9 / 2}^{\circ}$ & SO & 0.88 & 0.25 & & 4129.530 & 20 & 24209.00 & & $\Delta \mathrm{J}=0$ & 1.22 & 0.22 \\
\hline 4179.486 & 20 & 23919.65 & $18990_{11 / 2}-42910^{\circ}{ }_{13 / 2}$ & $\mathrm{~T}$ & 1.20 & & & 4128.477 & 20 & 24215.18 & $18837_{9 / 2}-43052^{\circ}{ }_{7 / 2}$ & & & \\
\hline 4178.247 & $4 h$ & 23926.74 & $19748_{9 / 2}-43675_{9 / 2}^{\circ}$ & & & & & 4128.168 & 20 & 24216.99 & $18693_{15 / 2}-42910^{\circ}{ }_{13 / 2}$ & So & 1.02 & 0.16 \\
\hline 4177.083 & 7 & 23933.41 & $18837_{9 / 2}-42770_{9 / 2}^{\circ}$ & $\Delta \mathrm{J}=0$ & 1.17 & 0.38 & & 4127.408 & 2 & .45 & & & & \\
\hline 4175.528 & 3 & 23942.32 & $19466_{13 / 2}-43408_{11 / 2}^{\circ}$ & & & & & 097 & 2 & 242 & & & & \\
\hline 4173.235 & 6 & 23955.47 & $17613_{9 / 2}-41569^{\circ}{ }_{11 / 2}$ & SI & 1.25 & 0.05 & & 4126.612 & $20 h$ & 24226.12 & $19748_{9 / 2}-43974_{11 / 2}^{\circ}$ & & & \\
\hline 4172.010 & 7 & 23962.51 & $17613_{9 / 2}-41576_{9 / 2}^{\circ}$ & $\Delta \mathrm{J}=0$ & 1.22 & 0.23 & & 4126.298 & 200 & 24227.96 & $17613_{9 / 2}^{/ / 2}-41841^{\circ / 2}{ }_{7 / 2}^{\circ}$ & so & 1.15 & 0.04 \\
\hline 4171.460 & 2 & 23965.67 & & & & & & 4125.476 & 80 & 24232.79 & $17343_{7 / 2}-41576_{9 / 2}^{\circ}$ & SI & 1.02 & 1.25 \\
\hline 4170.790 & 1 & 23969.52 & & & & & & 4.648 & $10 h$ & 242 & & & & \\
\hline 4170.449 & 500 & 23971.48 & $18990_{11 / 2}-42961^{\circ}{ }_{11 / 2}$ & $\Delta \mathrm{J}=0$ & 1.20 & 0.08 & & 4122.979 & $20 h$ & 24247.47 & $19753_{\tau / 2}-44001_{9 / 2}^{\circ}$ & & & \\
\hline 4169.232 & 5 & 23978.47 & $24137_{13 / 2}-48115^{\circ}{ }_{13 / 2}$ & & & & & 4122.081 & 4 & 24252.75 & $19748_{9 / 2}-44001_{9 / 2}^{\circ}$ & & & \\
\hline 4168.720 & 300 & 23981.42 & $19132_{3 / 2}-43113^{\circ}{ }_{5 / 2}$ & SI & 0.88 & 1.11 & * & 4120.725 & 20 & 24260.73 & $17613_{9 / 2}-41874^{\circ}{ }_{11 / 2}^{1 / 2}$ & So & 1.07 & 0.11 \\
\hline 4168.535 & $200 h$ & 23982.48 & $17343_{7 / 2}-41325^{\circ}{ }_{s / 2}$ & & & & & 4120.171 & 1 & 24263.99 & & & & \\
\hline 4167.970 & 3 & 23985.73 & & & & & & .475 & 50 & 24268.09 & $17613_{\vartheta_{/ 2}}-41881_{9 / 2}^{\circ}$ & $\mathrm{T}$ & 1.19 & 0.04 \\
\hline 4167.092 & 4 & 23990.79 & $17752_{s / 2}-41743_{3 / 2}^{\circ}$ & SI & 1.184 & 0.91 & * & 4119.262 & 3 & 24269.35 & & & & \\
\hline 4165.764 & 2 & 23998.44 & & & & & & 4119.124 & 30 & 24270.16 & $16742_{\tau / 2}-41012^{\circ}{ }_{7 / 2}$ & $\Delta \mathrm{J}=0$ & 1.23 & 0.63 \\
\hline .495 & 5 & 23999.99 & & & & & & 4116.710 & 3 & 24284.39 & & & & \\
\hline 4165.125 & 20 & 24002.12 & $18837_{9 / 2}-42839_{9 / 2}^{\circ}$ & $\Delta \mathrm{J}=0$ & 1.10 & 0.20 & & 5.834 & $20 h$ & 2428 & & & & \\
\hline 4163.708 & 3 & 24010.29 & & & & & & 4115.764 & 6 & 24289.97 & & & & \\
\hline 3.030 & 3 & 24014.20 & & & & & & 4114.592 & 6 & 89 & & & & \\
\hline 4161.735 & 80 & 24021.67 & $16742_{7 / 2}-40763_{7 / 2}^{\circ}$ & $\Delta \mathrm{J}=0$ & 1.32 & 1.175 & $*$ & 4114.320 & 100 & 24298.50 & $19132_{3 / 2}-43430^{\circ}{ }_{s / 2}$ & SI & 1.40 & 0.32 \\
\hline 4159.870 & $20 h$ & 24032.44 & $17613_{9 / 2}-41646^{\circ}{ }_{7 / 2}$ & & & & & 4114.128 & $100 h$ & 24299.63 & & & & \\
\hline 4158.665 & 8 & 24039.40 & & & & & & 4111.853 & 5 & 24313.08 & & & & \\
\hline 4158.601 & $500 r$ & 24039.77 & $8771_{s / 2}^{\circ}-32811_{7 / 2}$ & SI & 0.85 & 1.04 & * & 4111.208 & 3 & 24316.89 & & & & \\
\hline & 3 & 24053.11 & & & & & & 4109.328 & $5 h$ & 243 & & & & \\
\hline 898 & 3 & 24055.41 & $16957_{\tau / 2}-41012_{7 / 2}^{\circ}$ & & & & & 4108.565 & 2 & 24332.53 & $18853_{11 / 2}-43186_{11 / 2}^{\circ}$ & & & \\
\hline 4155.757 & 30 & 24056.22 & $18853_{11 / 2}-42910^{\circ}{ }_{13 / 2}$ & SI & 1.47 & 0.27 & & 4107.930 & 300 & 24336.29 & $16742_{7 / 2}-41078_{9 / 2}^{\circ}$ & $\mathrm{T}$ & 1.32 & \\
\hline 4154.720 & 2 & 24062.23 & & & & & & 4107.531 & 1 & 243 & & & & \\
\hline 4154.130 & $300 h$ & 24065.64 & & & & & & 4106.756 & 5 & 24343.25 & $20406_{\mathrm{s} / 2}^{\circ}-44750_{7 / 2}$ & so & 1.08 & 1.08 \\
\hline .430 & $20 h$ & 24069.70 & & & & & & 4105.841 & $50000 a$ & 24348.67 & $0_{7_{/ 2}}^{\circ}-24348_{9 / 2}$ & so & 1.05 & 0.07 \\
\hline 4152.732 & 20 & 24073.75 & $17613_{9 / 2}-41687^{\circ}{ }_{11 / 2}$ & so & 1.01 & 0.13 & & 4105.010 & 5 & 24353.60 & & & & \\
\hline 4151.108 & 2 & 24083.16 & & & & & & 4104.697 & $3 h$ & 24355.46 & & & & \\
\hline 4150.104 & 200 & 24088.99 & $17752_{s / 2}-41841^{\circ}{ }_{7 / 2}$ & SI & 1.25 & 0.05 & & 4104.189 & 20 & 24358.48 & $18693_{15 / 2}-43051^{\circ}{ }_{15 / 2}$ & $\mathrm{~T}$ & 1.185 & \\
\hline 4149.144 & 300 & 240 & & $\mathrm{~T}$ & 1.10 & 0.04 & & 4103.792 & 6 & 24360.83 & & & & \\
\hline 4148.910 & $5 h$ & 24095.92 & & & & & & 4102.727 & 60 & 24367.16 & $16742_{7 / 2}-41109_{9 / 2}^{\circ}$ & so & 0.68 & 0.51 \\
\hline 4148.475 & $20 h$ & 24098.45 & $19132_{3 / 2}-43230_{3 / 2}^{\circ}$ & $\Delta \mathrm{J}=0$ & 0.93 & 0.17 & & 4101.460 & $10 h$ & 24374.68 & & & & \\
\hline 4148.222 & $10 h$ & 24099.92 & & & & & & 4100.458 & 10 & 24380.64 & $17752_{s / 2}-42133_{7 / 2}^{\circ}$ & $\mathrm{T}$ & 1.18 & \\
\hline 4147.890 & $20 d$ & 24101.85 & $13119_{9 / 2}-37221^{\circ}{ }_{7 / 2}$ & & & & & 4100.340 & 3 & 24381.34 & & & & \\
\hline 4147.178 & 10 & 24105.98 & & & & & & 4100.217 & 8 & 24382.07 & $16742_{7 / 2}-41124_{9 / 2}^{\circ}$ & SI & 1.41 & 0.24 \\
\hline 4146.821 & 60 & 24108.06 & $18853_{11 / 2}-42961^{\circ}{ }_{11 / 2}$ & & & & & 4100.171 & 10 & 24382.35 & 5 & & & \\
\hline 4146.260 & 3 & 24111.32 & & & & & & 4099.780 & 2 & 24384.67 & $23374_{13 / 2}-47759^{\circ}{ }_{13 / 2}$ & & & \\
\hline 4146.129 & $40 h$ & 24112.08 & $20406_{s / 2}^{\circ}-44518_{s / 2}$ & $\Delta \mathrm{J}=0$ & 1.58 & 1.21 & * & 4099.127 & 10 & 24388.56 & $19753_{7 / 2}-44142^{\circ}{ }_{7 / 2}^{1 / 2}$ & $\Delta \mathrm{J}=0$ & 1.10 & 0.57 \\
\hline 4145.910 & 40 & 24113.36 & $16742_{7 / 2}^{/ 2}-4.0855_{9 / 2}^{\circ / 2}$ & So & 1.325 & 1.08 & $*$ & 4097.747 & $10 h$ & 24396.77 & & & & \\
\hline 414 & 1 & 24114.30 & 174 & & & & & 4094.187 & $50000 a$ & 24417.98 & $0_{7 / 2}^{\circ}-24$ & SI & 1.30 & 0.20 \\
\hline 4144.553 & 5 & 24121.25 & $15587_{11 / 2}-39709^{\circ}{ }_{11 / 2}^{1 / 2}$ & & & & & 4092.631 & 30 & 24427.27 & $17752_{s / 2}^{1 / 2}-42179_{\mathrm{s} / 2}^{\circ}$ & $\Delta \mathrm{J}=0$ & 1.12 & 0.35 \\
\hline 4144.325 & 50 & 24122.58 & $20406_{s / 2}^{\circ / 2}-44529_{7 / 2}$ & SoO & 1.58 & 0.97 & * & 4092.425 & 50 & 24428.49 & $19753_{\tau^{\prime 2}}-44182_{\mathrm{s} / 2}^{\circ}$ & SI & 1.25 & 0.06 \\
\hline 4143.995 & 100 & 24124.50 & $18837_{9 / 2}-42961^{\circ / 2} 11 / 2$ & & & & & 4092.372 & 5 & 24428.81 & & & & \\
\hline 4141.704 & 4 & 24137.84 & & & & & & 4091.771 & 80 & 24432.40 & $19748_{9 / 2}-44180^{\circ}$ & so & 0.57 & 0.54 \\
\hline 4141.495 & $60 h$ & 24139.06 & $20406_{s / 2}^{\circ}-44545_{3 / 2}$ & SI & 1.58 & 1.10 & * & 4091.355 & 30 & 24434.88 & $18853_{11 / 2}^{/ 2}-43288^{\circ}{ }_{13 / 2}$ & SI & 1.32 & 0.14 \\
\hline 4141.098 & 3 & 24141.38 & & & & & & 4090.672 & 5 & 24438.96 & $18990_{11 / 2}^{1 / 2}-43429_{9 / 2}^{\circ}$ & & & \\
\hline 4140.596 & $70 h$ & 24144.30 & & & & & & 4089.342 & $6 h$ & 24446.91 & $16742_{7 / 2}-41189^{\circ}{ }_{7 / 2}^{\circ / 2}$ & & & \\
\hline 4140.030 & 80 & 24147.60 & $19466_{13 / 2}-43614^{\circ}{ }_{15 / 2}$ & so & 0.80 & 0.30 & & 4089.187 & 30 & 24447.84 & $18990_{11 / 2}-43438^{\circ}{ }_{13 / 2}$ & $\mathrm{~T}$ & 1.20 & 0.04 \\
\hline 4138.387 & $400 r$ & 24157.19 & $13119_{9 / 2}-37276^{\circ}{ }_{11 / 2}^{1 / 2}$ & so & 0.98 & 0.25 & & 4088.927 & 4 & 24449.39 & $23309_{11 / 2}-47759^{13 / 2}$ & & & \\
\hline
\end{tabular}


APPENDIX - Spectral Lines of Neutral Thulium-Continued

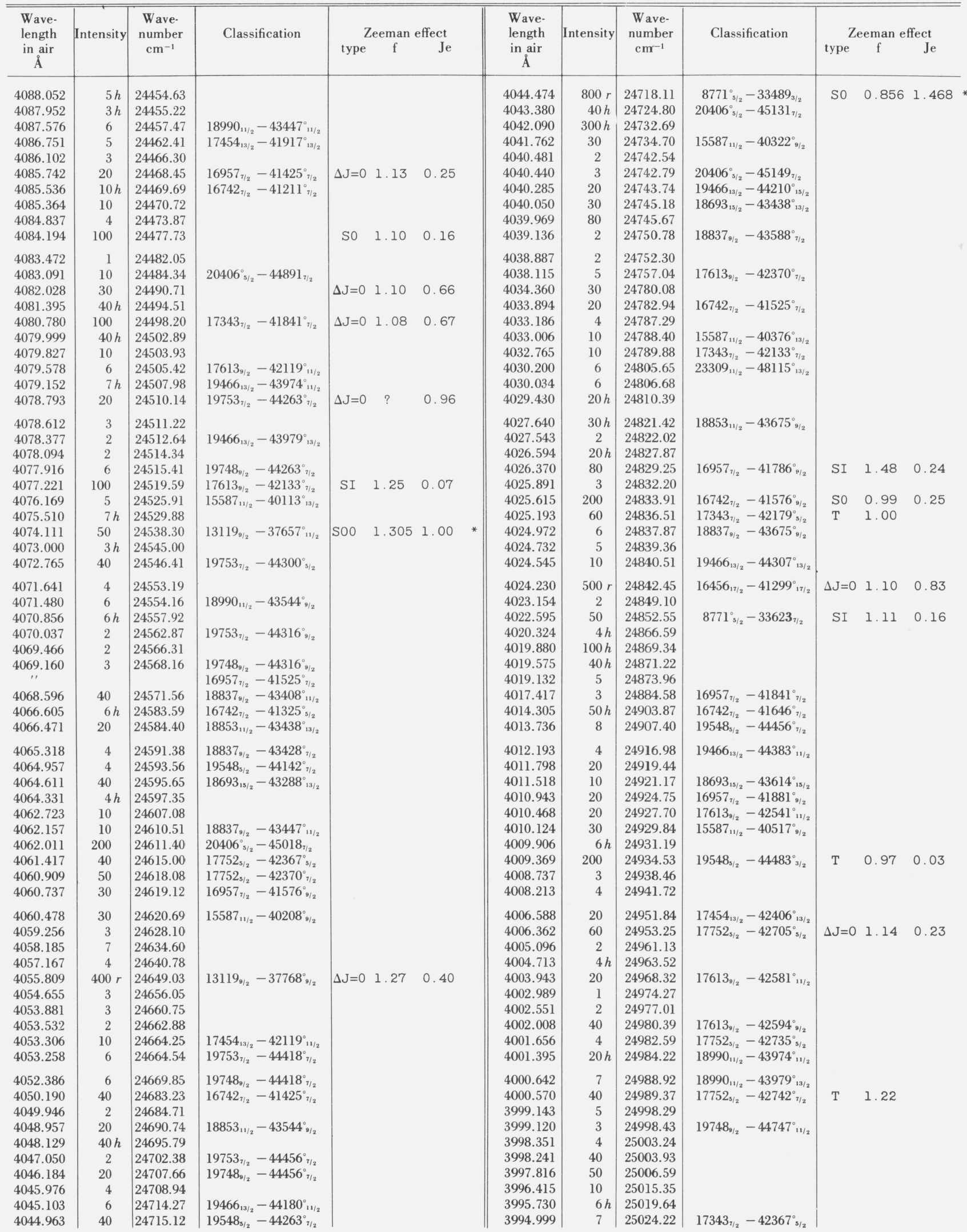


APPENDIX-Spectral Lines of Neutral Thulium-Continued

\begin{tabular}{|c|c|c|c|c|c|c|c|c|c|c|c|c|c|}
\hline \multirow{2}{*}{$\begin{array}{c}\text { Wave- } \\
\text { length } \\
\text { in air } \\
\AA\end{array}$} & \multirow[t]{2}{*}{ Intensity } & \multirow{2}{*}{$\begin{array}{l}\text { Wave- } \\
\text { number } \\
\mathrm{cm}^{-1}\end{array}$} & \multirow[t]{2}{*}{ Classification } & \multicolumn{3}{|c|}{ Zeeman effect } & \multirow{2}{*}{$\begin{array}{c}\text { Wave- } \\
\text { length } \\
\text { in air } \\
\AA\end{array}$} & Intensity & $\begin{array}{c}\text { Wave- } \\
\text { number }\end{array}$ & Classification & & eeman $\mathrm{e}$ & ffect \\
\hline & & & & type & & & & & $\mathrm{cm}^{-1}$ & & type & f & $\mathrm{Je}$ \\
\hline 3994.503 & 20 & 25027.33 & $17343_{\tau / 2}-42370_{7 / 2}^{\circ}$ & & & & 3939.089 & 80 & 25379.40 & $13119_{9 / 2}-38499_{9 / 2}^{\circ}$ & & & \\
\hline 3993.352 & 3 & 25034.54 & & & & & 3937.282 & 100 & 25391.04 & $16742_{7 / 2}-42133_{7 / 2}^{\circ}$ & $\Delta \mathrm{J}=0$ & 1. 22 & 0.54 \\
\hline 3991.840 & $20 h$ & 25044.02 & $16742_{\tau / 2}-41786_{9 / 2}^{\circ}$ & & & & 3937.159 & 2 & 25391.84 & $17343_{7 / 2}-42735_{5 / 2}^{\circ / 2}$ & & & \\
\hline 3990.875 & 50 & 25050.08 & $19132_{3 / 2}-44182^{\circ}{ }_{5 / 2}^{\circ}$ & & & & 3936.939 & 4 & 25393.26 & $18990_{11 / 2}^{1 / 2}-44383_{11 / 2}^{\circ / 2}$ & & & \\
\hline 3989.289 & 2 & 25060.04 & & & & & 3936.100 & 30 & 25398.67 & $17343_{\tau^{2}}-42742^{\circ}{ }^{\circ / 2}$ & & & \\
\hline 3987.865 & 3 & 25068.98 & & & & & 3935.799 & 80 & 25400.61 & $17454_{13 / 2}-42855_{15 / 2}^{\circ / 2}$ & $\mathrm{~T}$ & 1.125 & \\
\hline 3987.605 & $7 h$ & 25070.62 & & & & & 3934.332 & 10 & 25410.08 & $13119_{9 / 2}-38529^{\circ}{ }_{11 / 2}^{\circ / 2}$ & & & \\
\hline 3986.861 & 4 & 25075.30 & $15587_{11 / 2}-40663^{\circ}{ }_{11 / 2}$ & & & & 3934.252 & 6 & 25410.60 & $16957_{7 / 2}-42367^{\circ}{ }^{1 / 2}$ & & & \\
\hline 3985.764 & 6 & 25082.20 & $18990_{11 / 2}-44072^{\circ}{ }_{13 / 2}$ & & & & 3934.112 & 2 & 25411.50 & & & & \\
\hline 3985.074 & 10 & 25086.54 & $17454_{13 / 2}-42541^{\circ}{ }_{11 / 2}^{\circ / 2}$ & & & & 3933.767 & 20 & 25413.73 & $16957_{7 / 2}-42370^{\circ}{ }_{7 / 2}$ & & & \\
\hline 3984.747 & 3 & 25088.60 & & & & & 3932.910 & 3 & 25419.27 & & & & \\
\hline 3984.207 & 15 & 25092.00 & $13119_{9 / 2}-38211^{\circ}{ }_{7 / 2}$ & & & & 3932.718 & 20 & 25420.51 & $17613_{9 / 2}-43034_{7 / 2}^{\circ}$ & & & \\
\hline 3983.676 & $2 h$ & 25095.35 & & & & & 3932.300 & 4 & 25423.21 & $16456_{17 / 2}-41880_{15 / 2}^{\circ}$ & & & \\
\hline 3983.040 & 10 & 25099.35 & $16742_{7 / 2}-41841_{7 / 2}^{\circ}$ & & & & 3931.775 & 2 & 25426.61 & $18837_{9 / 2}-44263^{\circ}{ }_{7 / 2}^{\circ / 2}$ & & & \\
\hline 3982.716 & 15 & 25101.39 & $19753_{7 / 2}-44855_{\mathrm{s} / 2}^{\circ}$ & & & & 3931.649 & 20 & 25427.42 & $17343_{7 / 2}-42770_{9 / 2}^{\circ}$ & & & \\
\hline 3982.321 & 3 & 25103.88 & & & & & 3931.514 & 15 & 25428.29 & $18990_{11 / 2}^{1 / 2}-44418^{\circ / 2}{ }_{13 / 2}^{0}$ & & & \\
\hline 3980.918 & 3 & 25112.73 & $21737_{9 / 2}-46850^{\circ}{ }_{11 / 2}$ & & & & 3930.717 & 10 & 25433.45 & $25130_{15 /}-50563_{15 / 2}^{\circ}$ & & & \\
\hline 3980.661 & 40 & 25114.35 & $19753_{7 / 2}-44868_{9 / 2}^{\circ}$ & & & & 3930.066 & 20 & 25437.66 & $16742_{\tau_{/ 2}}-42179^{\circ}{ }_{s / 2}$ & & & \\
\hline 3979.825 & 30 & 25119.63 & $19748_{9 / 2}-44868_{9 / 2}^{\circ}$ & & & & 3927.639 & 2 & 25453.38 & $18853_{11 / 2}-44307^{\circ}{ }_{13 / 2}$ & & & \\
\hline 3979.637 & $20 h$ & 25120.81 & $18853_{11 / 2}-43974_{11 / 2}^{\circ / 2}$ & & & & 3927.355 & 20 & 25455.22 & $17454_{13 / 2}-42910^{\circ}{ }_{13 / 2}$ & & & \\
\hline 3979.062 & 3 & 25124.44 & $19466_{13 / 2}-44591^{\circ}{ }_{13 / 2}$ & & & & 3926.742 & 5 & 25459.20 & & & & \\
\hline 3978.897 & 10 & 25125.49 & $18853_{11 / 2}-43979^{\circ}{ }_{13 / 2}^{\circ / 2}$ & & & & 3925.096 & 3 & 25469.87 & & & & \\
\hline 3978.635 & 40 & 25127.14 & $17454_{13 / 2}-42581^{\circ}{ }_{11 / 2}^{\circ / 2}$ & & & & 3923.638 & 8 & 25479.34 & $18837_{9 / 2}-44316_{9 / 2}^{\circ}$ & & & \\
\hline 3978.447 & 3 & 25128.33 & $17613_{9 / 2}-42742^{\circ}{ }_{\eta / 2}$ & & & & 3922.405 & $1 h$ & 25487.35 & $21997_{11 / 2}-47484^{\circ / 2}{ }_{11 / 2}$ & & & \\
\hline 3977.032 & $100 h$ & 25137.27 & $18837_{9 / 2}-43974^{\circ}{ }_{11 / 2}$ & & & & 3921.885 & $5 h$ & 25490.72 & $15587_{11 / 2}^{1 / 2}-41078_{9 / 2}^{\circ}$ & & & \\
\hline 3976.679 & 100 & 25139.50 & $16742_{7 / 2}-41881_{9 / 2}^{\circ}$ & So & 0.63 & 0.50 & 3921.446 & 60 & 25493.58 & $15587_{11 / 2}-41081^{\circ}{ }_{11 / 2}$ & $\Delta \mathrm{J}=0$ & 1. 28 & 0.20 \\
\hline 3975.425 & 6 & 25147.43 & $18853_{11 / 2}^{/ 2}-44001_{9 / 2}^{\circ}$ & & & & 3921.056 & 2 & 25496.11 & $17343_{\tau / 2}^{1 / 2}-42839^{\circ}{ }_{9 / 2}^{1 / 2}$ & & & \\
\hline 3975.040 & $3 h$ & 25149.87 & $17752_{\mathrm{s} / 2}-42902_{\mathrm{s} / 2}^{\circ}$ & & & & 3919.372 & 40 & 25507.07 & $17454_{13 / 2}^{1 / 2}-42961_{11 / 2}^{\circ / 2}$ & & & \\
\hline 3973.894 & 10 & 25157.12 & $17613_{9 / 2}-42770^{\circ}{ }_{9 / 2}$ & & & & 3919.023 & 2 & 25509.34 & & & & \\
\hline 3973.200 & 2 & 25161.51 & & & & & 3918.934 & 10 & 25509.92 & $15271_{15 / 2}-40780^{\circ}{ }_{13 / 2}$ & & & \\
\hline 3972.828 & 2 & 25163.87 & $18837_{9 / 2}-44001_{9 / 2}^{\circ}$ & & & & 3918.376 & 3 & 25513.55 & & & & \\
\hline 3972.174 & 3 & 25168.01 & $19132_{3 / 2}-44300_{s / 2}^{\circ}$ & & & & 3917.144 & 40 & 25521.58 & $15587_{11 / 2}-41109_{9 / 2}^{\circ}$ & & & \\
\hline 3971.232 & $20 h$ & 25173.98 & & & & & 3916.477 & $5000 r$ & 25525.92 & $8771_{s / 2}^{\circ / 2}-34297_{7 / 2}^{7 / 2}$ & $\mathrm{~T}$ & 0.94 & \\
\hline 3970.878 & 20 & 25176.23 & $16957_{\tau / 2}-42133_{\tau_{1 / 2}}^{\circ}$ & & & & 3914.850 & 6 & 25536.53 & $15587_{11 / 2}-41124_{9 / 2}^{\circ / 2}$ & & & \\
\hline 3970.138 & 7 & 25180.92 & & & & & 3914.452 & $8 h$ & 25539.13 & & & & \\
\hline 3970.090 & 4 & 25181.22 & & & & & 3913.619 & 40 & 25544.56 & $13119_{9 / 2}-38664^{\circ}{ }_{7 / 2}$ & & & \\
\hline 3968.621 & 50 & 25190.54 & $18990_{11 / 2}-44180^{\circ}{ }_{11 / 2}$ & $\Delta \mathrm{J}=0$ & 1.18 & 0.25 & 3913.528 & 3 & 25545.16 & & & & \\
\hline 3968.222 & 20 & 25193.08 & $15587_{11 / 2}-40780^{\circ}{ }_{13 / 2}$ & & & & 3913.360 & 30 & 25546.25 & $18837_{9 / 2}-44383^{\circ}{ }_{11 / 2}$ & & & \\
\hline 3964.804 & 15 & 25214.79 & & & & & 3911.384 & 10 & 25559.16 & $17343_{7 / 2}-42902^{\circ}{ }_{s / 2}^{\circ / 2}$ & & & \\
\hline 3964.484 & 10 & 25216.83 & $21997_{11 / 2}-47214^{\circ}{ }_{13 / 2}$ & & & & 3910.504 & 40 & 25564.91 & $18853_{11 / 2}-44418^{\circ}{ }_{13 / 2}$ & & & \\
\hline 3964.172 & 20 & 25218.81 & $18853_{11 / 2}-44072^{\circ}{ }_{13 / 2}$ & & & & 3910.346 & 10 & 25565.94 & $13119_{9 / 2}-38685_{9 / 2}^{\circ}$ & & & \\
\hline 3963.532 & 50 & 25222.89 & $16957_{\tau^{\prime 2}}-42179_{\mathrm{s} / 2}^{\circ}$ & SI & 1.42 & 0.30 & 3910.200 & 3 & 25566.90 & & & & \\
\hline 3963.068 & 10 & 25225.84 & $17613_{9 / 2}^{/ 2}-42839_{9 / 2}^{\circ}$ & & & & 3909.315 & 10 & 25572.69 & $17613_{9 / 2}-43186_{11 / 2}^{\circ}$ & & & \\
\hline 3962.686 & 60 & 25228.27 & $13119_{9 / 2}-38347_{11 / 2}^{\circ / 2}$ & & & & 3906.478 & 2 & 25591.26 & & & & \\
\hline 3960.192 & 2 & 25244.16 & & & & & 3905.032 & 2 & 25600.73 & $18990_{11 / 2}-44591^{\circ}{ }_{13 / 2}$ & & & \\
\hline 3959.427 & $30 h$ & 25249.04 & & & & & 3904.452 & 3 & 25604.54 & $16957_{7 / 2}-42561_{9 / 2}^{\circ / 2}$ & & & \\
\hline 3959.168 & 20 & 25250.69 & $17343_{7 / 2}-42594_{9 / 2}^{\circ}$ & & & & 3903.098 & 2 & 25613.42 & & & & \\
\hline 3956.878 & 60 & 25265.30 & $13119_{9 / 2}^{/ 2}-38384_{9 / 2}^{\circ / 2}$ & & & & 3902.944 & 4 & 25614.43 & & & & \\
\hline 3956.492 & 20 & 25267.77 & $15587_{11 / 2}^{/ 2}-40855_{9 / 2}^{\circ / 2}$ & & & & 3902.268 & 20 & 25618.87 & $18837_{9 / 2}-44456^{\circ}{ }_{7 / 2}$ & & & \\
\hline 3954.520 & 7 & 25280.37 & $19466_{13 / 2}-44747^{\circ}{ }_{11 / 2}$ & & & & 3901.276 & 4 & 25625.38 & $16742_{7 / 2}-42367^{\circ}{ }_{5 / 2}^{\circ}$ & & & \\
\hline 3953.600 & 2 & 25286.25 & $18693_{15 / 2}-43979^{\circ}{ }_{13 / 2}$ & & & & 3899.496 & 20 & 25637.08 & $16957_{7 / 2}-42594_{9 / 2}^{\circ}$ & & & \\
\hline 3952.760 & $4 h$ & 25291.62 & & & & & 3896.617 & $1000 r$ & 25656.02 & $0^{\circ}{ }_{7 / 2}-25656_{5 / 2}^{/ 2}$ & SI & 1.14 & 0.72 \\
\hline 3951.466 & 4 & 25299.90 & $17752_{5 / 2}-43052^{\circ}{ }_{7 / 2}$ & & & & 3895.993 & 40 & 25660.13 & $19748_{9 / 2}-45408^{\circ / 2}{ }_{7 / 2}$ & & & \\
\hline 3949.920 & 5 & 25309.81 & $19748_{\mathrm{g} / 2}^{/ 2}-45058^{\circ}$ & & & & 3895.418 & 4 & 25663.91 & & & & \\
\hline 3949.270 & $3000 r$ & 25313.97 & $8771_{s_{s / 2}}^{\circ}-34085_{s / 2}$ & $\Delta \mathrm{J}=0$ & 0.90 & 0.24 & 3893.525 & 7 & 25676.39 & & & & \\
\hline $\begin{array}{l}3948.833 \\
\quad, "\end{array}$ & 20 & 25316.77 & $\begin{array}{l}19753_{7 / 2}-45070^{\circ}{ }_{9 / 2} \\
18990_{11 / 2}-44307^{\circ}{ }_{13 / 2}\end{array}$ & & & & $\begin{array}{l}3893.257 \\
3892.673\end{array}$ & $\begin{array}{r}10 \\
6\end{array}$ & $\begin{array}{l}25678.16 \\
25682.01\end{array}$ & $17752_{s / 2}-43430_{s / 2}^{\circ}$ & & & \\
\hline 3948.023 & 4 & 25321.97 & & & & & 3891.900 & 5 & 25687.11 & $15587_{11 / 2}-41274^{\circ}{ }_{13 / 2}$ & & & \\
\hline 3947.336 & 3 & 25326.37 & $18990_{11 / 2}-44316_{9 / 2}^{\circ}$ & & & & 3889.880 & $5 h$ & 25700.45 & & & & \\
\hline 3947.222 & 60 & 25327.10 & $18853_{11 / 2}-44180^{\circ}{ }_{11 / 2}$ & $\mathrm{~T}$ & 1.15 & & 3888.558 & 30 & 25709.19 & $17343_{7 / 2}-43052^{\circ}{ }_{7 / 2}$ & $\Delta \mathrm{J}=0$ & 1.04 & 0.13 \\
\hline 3946.589 & 6 & 25331.17 & & & & & 3887.842 & 3 & 25713.92 & $19753_{\tau_{/ 2}}-45467_{9 / 2}^{\circ}$ & & & \\
\hline 3945.650 & 5 & 25337.20 & $19466_{13 / 2}-44803^{\circ}{ }_{13 / 2}$ & & & & 3887.348 & $5000 a$ & 25717.19 & $0_{7 / 2}^{\circ}-25717_{7 / 2}^{9 / 2}$ & $\Delta \mathrm{J}=0$ & 1.14 & 1.01 \\
\hline 3945.321 & 3 & 25339.31 & & & & & 3886.472 & 2 & 25722.99 & $19132_{3 / 2}-44855^{\circ}{ }_{3 / 2}$ & & & \\
\hline 3944.660 & 40 & 25343.55 & $18837_{9 / 2}-44180_{11 / 2}^{\circ}$ & SI & 1.32 & 0.18 & 3886.068 & 20 & 25725.66 & $18693_{15 / 2}^{/ 2}-44418^{\circ}{ }_{13 / 2}$ & & & \\
\hline 3943.935 & 80 & 25348.21 & $17613_{9 / 2}-42961^{\circ}{ }_{11 / 2}$ & $\mathrm{~T}$ & 1.24 & & 3885.885 & $4 h$ & 25726.87 & & & & \\
\hline 3943.490 & 80 & 25351.07 & $19132_{3 / 2}-44483_{3 / 2}^{\circ}$ & & & & 3885.726 & $2 h$ & 25727.93 & & & & \\
\hline 3941.946 & 15 & 25361.00 & $17752_{s / 2}-43113_{s / 2}^{\circ}$ & & & & 3885.133 & $5 h$ & 25731.85 & & & & \\
\hline 3941.710 & 40 & 25362.52 & $17343_{7 / 2}^{/ 2}-42705_{5 / 2}^{\circ / 2}$ & so & 0.83 & 0.18 & 3884.306 & 3 & 25737.33 & $18853_{11 / 2}-44591_{13 / 2}^{\circ}$ & & & \\
\hline 3941.640 & 30 & 25362.97 & $13119_{9 / 2}-38482^{\circ}{ }_{7 / 2}$ & & & & 3883.132 & $10000 a$ & 25745.11 & $0_{7 / 2}^{\circ}-25745_{5 / 2}^{\circ / 2}$ & SI & 1.25 & 0.13 \\
\hline 3939.993 & 3 & 25373.57 & & & & & 3882.559 & 4 & 25748.91 & $16957_{\tau_{/ 2}}-42705^{\circ}{ }_{s / 2}$ & & & \\
\hline
\end{tabular}


APPENDIX - Spectral Lines of Neutral Thulium-Continued

\begin{tabular}{|c|c|c|c|c|c|c|c|c|c|c|c|c|c|}
\hline \multirow{2}{*}{$\begin{array}{c}\text { Wave- } \\
\text { length } \\
\text { in air } \\
\AA\end{array}$} & \multirow[t]{2}{*}{ Intensity } & \multirow{2}{*}{$\begin{array}{l}\text { Wave- } \\
\text { number } \\
\mathrm{cm}^{-1}\end{array}$} & \multirow[t]{2}{*}{ Classification } & \multicolumn{3}{|c|}{ Zeeman effect } & \multirow{2}{*}{$\begin{array}{c}\text { Wave- } \\
\text { length } \\
\text { in air } \\
\AA\end{array}$} & Intensity & $\begin{array}{l}\text { Wave- } \\
\text { number }\end{array}$ & Classification & & eeman e & ffect \\
\hline & & & & type & & & & & & & type & & \\
\hline 3881.397 & 20 & 25756.62 & $18990_{11 / 2}-44747^{\circ}{ }_{11 / 2}$ & & & & 3824.430 & $100 h$ & 26140.27 & & $\mathrm{~T}$ & 1.17 & \\
\hline 3881.249 & 50 & 25757.60 & $13119_{9 / 2}-38877^{\circ}{ }_{11 / 2}$ & $\Delta \mathrm{J}=1$ & $?$ & 1.08 & 3824.326 & 2 & 26140.98 & & & & \\
\hline 3880.599 & 30 & 25761.92 & $21997_{11 / 2}-47759^{\circ}{ }_{13 / 2}$ & $\mathrm{~T}$ & 1.20 & & 3822.030 & 5 & 26156.68 & $16957_{\tau_{/ 2}}-43113_{5 / 2}^{\circ}$ & & & \\
\hline 3879.340 & 80 & 25770.28 & $17343_{\tau^{2} / 2}-43113_{5 / 2}^{\circ}$ & so & 0.80 & 0.22 & 3822.003 & 4 & 26156.87 & $18990_{11 / 2}^{1 / 2}-45147_{9 / 2}^{\circ / 2}$ & & & \\
\hline 3879.051 & 7 & 25772.20 & & & & & 3821.623 & $100 r$ & 26159.47 & $17454_{13 / 2}-43614^{\circ / 2}{ }_{15 / 2}^{1 / 2}$ & so & 0.98 & 0.16 \\
\hline 3878.143 & 5 & 25778.23 & $16957_{7 / 2}-42735_{5 / 2}^{\circ}$ & & & & 3821.511 & 5 & 26160.24 & $16742_{7 / 2}-42902_{5 / 2}^{\circ}$ & & & \\
\hline 3877.300 & 2 & 25783.84 & $18990_{11 / 2}-44774_{9 / 2}^{\circ}$ & & & & 3821.215 & 2 & 26162.26 & & & & \\
\hline 3877.126 & 10 & 25784.99 & $16957_{\tau_{/ 2}}-42742^{\circ}{ }_{7 / 2}$ & & & & 3819.906 & $10 h$ & 26171.23 & & & & \\
\hline 3876.864 & $2 h$ & 25786.74 & & & & & 3816.591 & 3 & 26193.96 & $19466_{13 / 2}-45660^{\circ}{ }_{13 / 2}$ & & & \\
\hline 3876.416 & 5 & 25789.72 & & & & & 3815.037 & $60 h$ & 26204.63 & & & & \\
\hline 3874.248 & 2 & 25804.15 & & & & & 3813.276 & 10 & 26216.73 & $18853_{11 / 2}-45070_{9 / 2}^{\circ}$ & & & \\
\hline 3873.824 & 4 & 25806.97 & & & & & 3810.888 & 10 & 26233.16 & $18837_{9 / 2}-45070_{9 / 2}^{\circ / 2}$ & & & \\
\hline 3873.446 & 30 & 25809.49 & & & & & 3810.055 & $30 h$ & 26238.89 & & & & \\
\hline 3873.400 & 2 & 25809.80 & $25488_{11 / 2}-51298^{\circ}{ }_{13 / 2}$ & & & & 3809.402 & 20 & 26243.39 & & & & \\
\hline 3872.846 & 40 & 25813.49 & $18990_{11 / 2}-44803^{\circ}{ }_{13 / 2}$ & & & & 3808.948 & 60 & 26246.52 & & $\mathrm{~T}$ & 1.14 & \\
\hline 3872.804 & 60 & 25813.77 & $16957_{7 / 2}-42770_{9 / 2}^{\circ}$ & & & & 3808.300 & 5 & 26250.98 & & & & \\
\hline 3872.512 & 200 & 25815.71 & $17613_{9 / 2}-43429_{9 / 2}^{\circ}$ & $\mathrm{T}$ & 1.18 & & 3807.719 & $700 r$ & 26254.99 & $8771_{5 / 2}^{\circ}-35026_{5 / 2}$ & $\Delta \mathrm{J}=0$ & 0.85 & 1.02 \\
\hline 3871.973 & 60 & 25819.31 & $16742_{7 / 2}^{/ 2}-42561_{9 / 2}^{\circ / 2}$ & & & & 3803.144 & 30 & 26286.57 & $15587_{11 / 2}-41874^{\circ}{ }_{11 / 2}^{1 / 2}$ & $\Delta \mathrm{J}=0$ & 1.21 & 0.46 \\
\hline 3871.846 & 30 & 25820.15 & & $\mathrm{~T}$ & 1.06 & & 3802.074 & $150 r$ & 26293.97 & $15587_{11 / 2}-41881_{9 / 2}^{\circ}$ & SI & 1.58 & 0.32 \\
\hline 3871.530 & 4 & 25822.26 & $19466_{13 / 2}-45288^{\circ}{ }_{11 / 2}$ & & & & 3799.874 & 3 & 26309.19 & & & & \\
\hline 3870.912 & $5 h$ & 25826.38 & & & & & 3799.774 & 5 & 26309.89 & $18837_{9 / 2}-45147_{9 / 2}^{\circ}$ & & & \\
\hline 3869.786 & 50 & 25833.90 & $17454_{13 / 2}-43288^{\circ}{ }_{13 / 2}$ & & & & 3799.710 & 30 & 26310.33 & $16742_{7 / 2}^{1 / 2}-43052^{\circ}{ }_{7 / 2}^{/ 2}$ & & & \\
\hline 3869.744 & 10 & 25834.18 & $17613_{9 / 2}-43447^{\circ}{ }_{11 / 2}$ & & & & 3798.541 & $800 r$ & 26318.43 & $8771_{5 / 2}^{\circ / 2}-35089_{3 / 2}^{/ 2}$ & $\mathrm{~T}$ & 0.85 & \\
\hline 3869.540 & 4 & 25835.54 & $17752_{5 / 2}-43588_{7 / 2}^{\circ}$ & & & & 3797.636 & 40 & 26324.70 & $13119_{9 / 2}^{/ 2}-39444_{9 / 2}^{\circ}$ & $\Delta \mathrm{J}=0$ & 1.20 & 0.83 \\
\hline 3867.107 & 8 & 25851.80 & $16742_{7 / 2}-42594_{9 / 2}^{\circ / 2}$ & & & & 3796.596 & 10 & 26331.91 & $17343_{7 / 2}-43675_{9 / 2}^{\circ}$ & & & \\
\hline 3865.909 & 5 & 25859.81 & $19548_{5 / 2}-45408^{\circ}{ }_{7 / 2}^{\circ / 2}$ & & & & 3795.965 & 3 & 26336.28 & $25130_{15 / 2}-51466^{\circ}{ }_{13 / 2}$ & & & \\
\hline 3863.972 & 70 & 25872.77 & $18693_{15 / 2}-44565^{\circ}{ }_{15 / 2}$ & $\Delta \mathrm{J}=0$ & 1.15 & 0.35 & 3794.872 & 3 & 26343.87 & $19466_{13 / 2}-45810^{\circ}{ }_{11 / 2}$ & & & \\
\hline 3863.524 & 3 & 25875.77 & & & & & 3793.269 & 10 & 26355.00 & & & & \\
\hline 3863.230 & 1 & 25877.74 & $18990_{11 / 2}-44868_{9 / 2}^{\circ}$ & & & & 3792.404 & $10 h$ & 26361.01 & $17613_{9 / 2}-43974_{11 / 2}^{\circ}$ & & & \\
\hline 3863.081 & 30 & 25878.74 & $18693_{15 / 2}-44571^{\circ}{ }_{17 / 2}$ & $\mathrm{~T}$ & 1.18 & & 3791.307 & 80 & 26368.64 & $13119_{9 / 2}-39488_{9 /}^{\circ}$ & $\Delta \mathrm{J}=0$ & 1.20 & 0.86 \\
\hline 3862.522 & 20 & 25882.48 & $16957_{\tau / 2}-42839_{9 / 2}^{\circ}$ & & & & 3790.905 & 20 & 26371.44 & $16742_{7 / 2}-43113_{5 / 2}^{\circ}$ & & & \\
\hline 3861.820 & 20 & 25887.19 & $19748_{9 / 2}-45635^{\circ}{ }_{11 / 2}$ & & & & 3788.759 & $5 h$ & 26386.37 & & & & \\
\hline 3860.925 & 5 & 25893.19 & $18853_{11 / 2}-44747^{\circ}{ }_{11 / 2}$ & & & & 3788.582 & 5 & 26387.61 & $17613_{9 / 2}-44001_{9 / 2}^{\circ}$ & & & \\
\hline 3860.201 & 2 & 25898.04 & $18693_{15 / 2}-44591^{\circ}{ }_{13 / 2}^{\circ / 2}$ & & & & 3788.273 & 2 & 26389.76 & $17752_{5 / 2}-44142^{\circ}{ }_{7 / 2}$ & & & \\
\hline 3858.480 & 100 & 25909.59 & $18837_{9 / 2}-44.747^{\circ}{ }_{11 / 2}$ & SI & 1.40 & 0.20 & 3787.605 & $10 h$ & 26394.41 & & & & \\
\hline 3856.868 & 15 & 25920.42 & $18853_{11 / 2}-44774_{9 / 2}^{\circ}$ & & & & 3787.014 & $120 r$ & 26398.53 & $16456_{17 / 2}-42855^{\circ}{ }_{15 / 2}$ & SI & 1.56 & 0.38 \\
\hline 3855.305 & 6 & 25930.93 & $17613_{9 / 2}^{/ 2}-43544_{9 / 2}^{\circ}$ & & & & 3784.645 & $2 h$ & 26415.06 & $19748_{9 / 2}-46163^{\circ}{ }_{11 / 2}$ & & & \\
\hline 3854.522 & $10 h$ & 25936.20 & & & & & 3784.327 & $4 h$ & 26417.28 & & & & \\
\hline 3854.426 & 20 & 25936.85 & $18837_{9 / 2}-44774_{9 / 2}^{\circ}$ & & & & 3783.855 & 2 & 26420.57 & & & & \\
\hline 3853.145 & 50 & 25945.47 & $16957_{\tau / 2}^{/ 2}-42902^{\circ}{ }_{5 / 2}$ & & & & 3782.550 & 30 & 26429.69 & $17752_{5 / 2}-44182_{5 / 2}^{\circ}$ & $\mathrm{T}$ & 1.20 & \\
\hline 3852.470 & 30 & 25950.01 & $18853_{11 / 2}-44803^{\circ}{ }_{13 / 2}$ & & & & 3781.148 & $200 a$ & 26439.48 & $0^{\circ}{ }_{\tau / 2}-26439_{\tau / 2}$ & $\Delta \mathrm{J}=0$ & 1.07 & 0.50 \\
\hline 3850.447 & 6 & 25963.65 & $16742_{7 / 2}-42705_{5 / 2}^{\circ}$ & & & & 3780.977 & 20 & 26440.68 & & & & \\
\hline 3849.518 & 6 & 25969.91 & $13119_{9 / 2}-39089^{\circ}{ }_{11 / 2}$ & & & & 3780.588 & 20 & 26443.40 & $13119_{9 / 2}-39563^{\circ}{ }_{11 / 2}$ & & & \\
\hline 3848.835 & 5 & 25974.52 & $17613_{9 / 2}-43588_{7 / 2}^{\circ}$ & & & & 3780.214 & 5 & 26446.02 & & & & \\
\hline 3848.295 & 5 & 25978.17 & $25488_{11 / 2}-51466_{13 / 2}^{\circ}$ & & & & 3779.414 & $60 h$ & 26451.62 & $18837_{9 / 2}-45289^{\circ}{ }_{11 / 2}$ & $\mathrm{~T}$ & 1.13 & \\
\hline 3847.836 & 20 & 25981.27 & $15587_{11 / 2}-41569^{\circ}{ }_{11 / 2}$ & & & & 3777.096 & 5 & 26467.85 & $19466_{13 / 2}-45934_{15 / 2}^{\circ}$ & & & \\
\hline 3847.514 & $100 r$ & 25983.44 & $17454_{13 / 2}-43438^{\circ}{ }_{13 / 2}$ & $\Delta \mathrm{J}=0$ & 1.22 & 0.53 & 3776.537 & 30 & 26471.77 & $16957_{\tau / 2}-43428^{\circ}{ }_{7 / 2}^{\circ / 2}$ & & & \\
\hline 3846.091 & 30 & 25993.05 & $17454_{13 / 2}-43447^{\circ}{ }_{11 / 2}^{\circ / 2}$ & & & & 3776.452 & 6 & 26472.36 & $16957_{\tau^{2}}-43429_{9 / 2}^{\circ}$ & & & \\
\hline 3845.100 & 5 & 25999.75 & $16742_{7 / 2}-42742_{7 / 2}^{\circ}$ & & & & 3773.864 & 20 & 26490.51 & $8771_{5 / 2}^{\circ / 2}-35261_{5 / 2}^{/ 2}$ & $\Delta \mathrm{J}=0$ & 0.855 & 1.346 \\
\hline 3842.946 & $150 h$ & 26014.32 & $18853_{11 / 2}-44868_{9 / 2}^{\circ}$ & & & & 3773.205 & 3 & 26495.14 & & & & \\
\hline 3840.869 & $500 r$ & 26028.39 & $15271_{15 / 2}-41299^{\circ}{ }_{17 / 2}$ & $\mathrm{~T}$ & 1.09 & & 3772.102 & $5 h$ & 26502.89 & & & & \\
\hline 3840.518 & 2 & 26030.77 & $18837_{9 / 2}-44868_{9 / 2}^{\circ}$ & & & & 3771.901 & $4 h$ & 26504.30 & & & & \\
\hline 3837.438 & $2 h$ & 26051.66 & & & & & 3769.693 & $100 h$ & 26519.82 & $17454_{13 / 2}-43974^{\circ}{ }_{11 / 2}$ & & & \\
\hline 3835.926 & 10 & 26061.93 & $19748_{9 / 2}-45810^{\circ}{ }_{11 / 2}$ & & & & 3769.032 & 6 & 26524.48 & $17454_{13 / 2}-43979^{\circ}{ }_{13 / 2}$ & & & \\
\hline 3835.051 & 20 & 26067.88 & $13119_{9 / 2}-39187^{\circ}{ }_{7 / 2}^{\circ}$ & & & & 3768.065 & 40 & 26531.28 & $15587_{11 / 2}-42119^{\circ}{ }_{11 / 2}$ & & & \\
\hline 3834.484 & 2 & 26071.73 & & & & & 3766.713 & 7 & 26540.81 & & & & \\
\hline 3834.262 & 7 & 26073.24 & $16957_{\tau / 2}-43030_{9 / 2}^{\circ}$ & & & & 3765.746 & 20 & 26547.62 & $17752_{5 / 2}-44300^{\circ}{ }_{5 / 2}$ & & & \\
\hline 3833.695 & 10 & 26077.10 & $16957_{\tau_{/ 2}}-43034_{\tau_{7 / 2}}^{\circ}$ & & & & 3764.387 & 8 & 26557.20 & & & & \\
\hline 3833.254 & 10 & 26080.10 & & & & & 3762.954 & 10 & 26567.32 & $17613_{9 / 2}-44180^{\circ}{ }_{11 / 2}$ & & & \\
\hline 3832.386 & 30 & 26086.00 & $17343_{\tau / 2}-43429_{9 / 2}^{\circ}$ & SI & 1.80 & 0.62 & 3762.352 & $150 h$ & 26571.57 & (1) & & & \\
\hline 3832.183 & 80 & 26087.39 & $17343_{7 / 2}-43430_{5 / 2}^{\circ}$ & so & 0.80 & 0.18 & 3759.820 & 40 & 26589.46 & $13119_{9 / 2}-39709^{\circ}{ }_{11 / 2}$ & so & 0.57 & 0.58 \\
\hline 3831.643 & 4 & 26091.06 & $19466_{13 / 2}-45557^{\circ}{ }_{15 / 2}$ & & & & 3759.086 & 40 & 26594.65 & $16456_{17 / 2}^{1 / 2}-43051^{\circ}{ }_{15 / 2}$ & $\mathrm{~T}$ & 1.15 & \\
\hline 3830.736 & 3 & 26097.24 & $16742_{7 / 2}-42839_{9 / 2}^{\circ}$ & & & & 3758.726 & 3 & 26597.20 & & & & \\
\hline 3830.394 & 30 & 26099.57 & $15587_{11 / 2}-41687^{\circ}{ }_{11 / 2}^{2}$ & & & & 3757.570 & 3 & 26605.38 & & & & \\
\hline 3830.267 & 30 & 26100.44 & $13119_{9 / 2}-39220_{9 / 2}^{\circ}$ & & & & 3757.040 & 6 & 26609.14 & $15271_{15 / 2}-4180^{\circ}{ }_{15 / 2}$ & & & \\
\hline 3829.065 & $30 h$ & 26108.63 & & $\mathrm{~T}$ & 1.10 & & 3756.370 & 10 & 26613.88 & $18853_{11 / 2}^{1 / 2}-45467^{\circ / 2}{ }_{9 / 2}$ & & & \\
\hline 3827.965 & $8 h$ & 26116.13 & & & & & 3755.818 & 4 & 26617.79 & $17454_{13 / 2}-44072^{\circ}{ }_{13 / 2}$ & & & \\
\hline 3827.666 & 4 & 26118.17 & $21997_{11 / 2}-48115_{13 / 2}^{\circ}$ & & & & 3754.055 & 10 & 26630.29 & $18837_{9 / 2}-45467_{9 / 2}^{\circ}$ & & & \\
\hline 3826.667 & 30 & 26124.99 & $13119_{9 / 2}-39244_{9 / 2}^{\circ}$ & & & & 3753.930 & 20 & 26631.18 & $16957_{7 / 2}^{1 / 2}-43588^{\circ}{ }_{7 / 2}$ & & & \\
\hline 3826.386 & $400 a$ & 26126.91 & $0^{\circ}{ }_{7 / 2}^{2}-26126_{5 / 2}^{/ 2}$ & SI & 1.22 & 0.09 & 3753.810 & 10 & 26632.03 & $18853_{11 / 2}-45485_{11 / 2}^{\circ / 2}$ & & & \\
\hline
\end{tabular}


APPENDIX - Spectral Lines of Neutral Thulium-Continued

\begin{tabular}{|c|c|c|c|c|c|c|c|c|c|c|c|c|c|}
\hline \multirow{2}{*}{$\begin{array}{c}\text { Wave- } \\
\text { length } \\
\text { in air } \\
\AA\end{array}$} & \multirow[t]{2}{*}{ Intensity } & \multirow{2}{*}{$\begin{array}{l}\text { W ave- } \\
\text { number } \\
\mathrm{cm}^{-1}\end{array}$} & \multirow[t]{2}{*}{ Classification } & \multicolumn{3}{|c|}{ Zeeman effect } & \multirow{2}{*}{$\begin{array}{c}\text { Wave- } \\
\text { length } \\
\text { in air } \\
\AA\end{array}$} & Intensity & $\begin{array}{c}\text { Wave- } \\
\text { number }\end{array}$ & Classification & & Zeeman & effect \\
\hline & & & & type & & $\mathrm{Je}$ & & & & & type & f & $\mathrm{Je}$ \\
\hline 3752.828 & $10 h$ & 26639.00 & & & & & 3675.029 & 30 & 27202.92 & $13119_{9 / 2}-40322 \%_{2}$ & $\Delta \mathrm{J}=0$ & 1.25 & 0.86 \\
\hline 3752.168 & 20 & 26643.69 & & & & & 3673.989 & 3 & 27210.62 & & & & \\
\hline 3751.806 & $2000 a$ & 26646.26 & $0_{7 / 2}^{\circ}-26646_{9 / 2}$ & so & 1.143 & $0.961 *$ & 3672.616 & $30 h$ & 27220.80 & & & & \\
\hline 3751.495 & 5 & 26648.47 & $18837_{9 / 2}-45485_{11 / 2}^{\circ}$ & & & & 3671.724 & 10 & 27227.41 & $19466_{13 / 2}-46694_{13 / 2}^{\circ}$ & $\mathrm{T}$ & 1.14 & \\
\hline 3748.433 & 3 & 26670.23 & $18990_{11 / 2}-45660^{\circ}{ }_{13 / 2}$ & & & & 3670.776 & 8 & 27234.44 & & & & \\
\hline 3746.208 & 5 & 26686.07 & & & & & 3669.835 & 3 & 27241.42 & $18693_{15 / 2}-45934^{\circ}{ }_{15 / 2}$ & & & \\
\hline 3746.139 & 3 & 26686.57 & $16742_{7 / 2}-43428_{7 / 2}^{\circ}$ & & & & 3669.081 & $20 h$ & 27247.02 & & & & \\
\hline 3746.057 & 20 & 26687.15 & $16742_{7 / 2}-43429^{\circ}$ & & & & 3668.452 & 30 & 27251.69 & $15587_{11 / 2}-42839_{9 / 2}^{\circ}$ & & & \\
\hline 3745.865 & 6 & 26688.52 & $16742_{\tau / 2}-43430^{\circ}{ }_{5 / 2}$ & & & & 3665.754 & 5 & 27271.75 & & & & \\
\hline 3744.954 & 8 & 26695.01 & & & & & 3664.617 & $10 h$ & 27280.21 & & & & \\
\hline 3744.064 & $6000 a$ & 26701.35 & $0_{7 / 2}^{\circ}-26701_{7 / 2}$ & $\Delta \mathrm{J}=0$ & 1.10 & 0.23 & 3663.005 & 90 & 27292.22 & $17454_{13 / 2}-44747^{\circ}{ }_{11 / 2}$ & so & 0.95 & 0.15 \\
\hline 3741.692 & 200 & 26718.28 & $16957_{\tau^{2}}-43675_{9 / 2}^{\circ}$ & So & 0.58 & 0.45 & 3662.276 & 10 & 27297.65 & & & & \\
\hline 3740.597 & 5 & 26726.10 & $17454_{13 / 2}-44180^{\circ}{ }_{11 / 2}$ & & & & 3661.200 & 2 & 27305.67 & & & & \\
\hline 3739.953 & 15 & 26730.70 & $17752_{5 / 2}-44483_{3 / 2}^{\circ}$ & & & & 3661.024 & 40 & 27306.98 & $16957_{7 / 2}-44263_{7 / 2}^{\circ}$ & & & \\
\hline 3739.772 & 100 & 26732.00 & & So & 0.70 & 0.30 & 3660.452 & 20 & 27311.25 & & $\mathrm{~T}$ & 1.36 & \\
\hline 3739.472 & 5 & 26734.14 & & & & & 3658.983 & 20 & 27322.22 & $15587_{11 / 2}-22910^{\circ}{ }_{13 / 2}$ & & & \\
\hline 3736.591 & 3 & 26754.75 & $19466_{13 / 2}-46221^{\circ}{ }_{11 / 2}$ & & & & 3658.008 & 3 & 27329.50 & $24137_{13 / 2}-51466^{\circ}{ }_{13 / 2}$ & & & \\
\hline 3736.471 & 40 & 26755.61 & $17454_{13 / 2}-44210^{\circ}{ }_{15 / 2}$ & $\mathrm{~T}$ & 1.15 & & 3656.196 & 5 & 27343.04 & & & & \\
\hline 3732.804 & 30 & 26781.90 & $18853_{11 / 2}-45635_{11 / 2}^{\circ}$ & $\mathrm{T}$ & 1.12 & & 3656.178 & 5 & 27343.18 & $16957_{\tau / 2}-44300_{5 / 2}^{\circ}$ & & & \\
\hline 3731.287 & 4 & 26792.79 & & & & & 3655.312 & 5 & 27349.65 & & & & \\
\hline 3731.048 & $3 h$ & 26794.50 & & & & & 3654.560 & $20 h$ & 27355.28 & & & & \\
\hline 3730.423 & 10 & 26798.99 & $17343_{\tau_{/ 2}}-44142^{\circ}{ }_{\tau / 2}$ & & & & 3653.968 & 30 & 27359.71 & $16957_{7 / 2}-44316_{9 / 2}^{\circ}$ & & & \\
\hline 3729.954 & 5 & 26802.36 & $16742_{7 / 2}-43544_{9 / 2}^{\circ}$ & & & & 3652.050 & $50 r$ & 27374.08 & $15587_{11 / 2}-42961^{\circ}{ }_{11 / 2}$ & $\Delta \mathrm{J}=0$ & 1.22 & 0.26 \\
\hline 3728.809 & $2 h$ & 26810.59 & & & & & 3651.557 & 4 & 27377.78 & 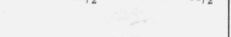 & & & \\
\hline 3727.487 & 20 & 26820.10 & $18990_{11 / 2}-45810^{\circ}{ }_{11 / 2}$ & & & & 3650.740 & $50 h$ & 27383.90 & & & & \\
\hline 3724.875 & 4 & 26838.91 & $17343_{7 / 2}-44182_{s / 2}^{\circ}$ & & & & 3649.784 & 3 & 27391.08 & & & & \\
\hline 3724.370 & 4 & 26842.54 & $17613_{9 / 2}-44456^{\circ}{ }_{\gamma_{/ 2}}$ & & & & 3648.853 & $50 r$ & 27398.07 & $13119_{9 / 2}-40517_{9 / 2}^{\circ}$ & $\Delta \mathrm{J}=0$ & 1.25 & 0.68 \\
\hline 3723.897 & 20 & 26845.95 & $16742_{7 / 2}-43588^{\circ}{ }_{7 / 2}^{\circ}$ & & & & 3648.572 & 5 & 27400.18 & $16742_{7 / 2}-44142^{\circ}{ }_{7 / 2}$ & & & \\
\hline 3723.005 & 5 & 26852.39 & $17454_{13 / 2}-44307^{\circ}{ }_{13 / 2}$ & & & & 3648.485 & 10 & 27400.83 & & & & \\
\hline 3722.400 & $70 h$ & 26856.75 & & & & & 3646.703 & $200 r$ & 27414.22 & $13119_{9 / 2}-40533_{7 / 2}^{\circ}$ & SI & 1.52 & 0.21 \\
\hline 3719.105 & 2 & 26880.54 & & & & & 3643.472 & 20 & 27438.53 & & & & \\
\hline 3717.914 & $10000 a$ & 26889.15 & $0_{\tau^{\prime} / 2}^{\circ}-26889_{9 / 2}$ & So & 1.00 & 0.10 & 3642.944 & $100 r$ & 27442.51 & $15587_{11 / 2}-43030_{9 / 2}^{\circ}$ & SI & 1.98 & 0.71 \\
\hline 3714.249 & 4 & 26915.69 & $18693_{15 / 2}-45608^{\circ}{ }_{15 / 2}$ & & & & 3641.034 & 10 & 27456.90 & $17613_{9 / 2}-45070_{9 / 2}^{\circ}$ & & & \\
\hline 3714.132 & 3 & 26916.53 & & & & & 3640.437 & 3 & 27461.40 & $16957_{7 / 2}-44418_{7 / 2}^{\circ}$ & & & \\
\hline 3713.573 & 10 & 26920.59 & $17343_{7 / 2}-44263^{\circ}{ }_{7 / 2}$ & & & & 3639.570 & 6 & 27467.94 & & & & \\
\hline 3712.860 & $6 h$ & 26925.76 & & & & & 3638.408 & $300 r$ & 27476.72 & $13119_{9 / 2}-40596_{7 / 2}^{\circ}$ & $\mathrm{T}$ & 1.31 & \\
\hline 3712.439 & 5 & 26928.81 & $17454_{13 / 2}-44383^{\circ}{ }_{11 / 2}$ & & & & 3633.763 & 20 & 27511.84 & $17343_{7 / 2}-44855_{5 / 2}^{\circ}$ & & & \\
\hline 3711.859 & $2 h$ & 26933.02 & $16742_{7 / 2}-43675_{9 / 2}^{\circ}$ & & & & 3632.062 & 50 & 27524.72 & & & & \\
\hline 3710.665 & 20 & 26941.68 & $13119_{9 / 2}-40061_{7 / 2}^{\circ}$ & & & & 3631.210 & $4 h$ & $2753(18$ & & & & \\
\hline 3709.030 & 20 & 26953.56 & $15587_{11 / 2}-42541_{11 / 2}^{\circ}$ & & & & 3630.895 & 4 & 27533.57 & & & & \\
\hline 3708.602 & 2 & 26956.67 & $18853_{11 / 2}-45810^{\circ}{ }_{11 / 2}$ & & & & 3629.876 & 7 & 27541.30 & & & & \\
\hline 3708.425 & $4 h$ & 26957.96 & & & & & 3627.598 & $10 h$ & 27558.59 & & & & \\
\hline 3707.099 & 30 & 26967.60 & $18693_{15 / 2}-45660_{13 / 2}$ & $\mathrm{~T}$ & 1.145 & & 3627.303 & 5 & 27560.83 & $19466_{13 / 2}-47027^{\circ}{ }_{13 / 2}$ & & & \\
\hline 3706.329 & 4 & 26973.20 & & & & & 3625.442 & $20 h$ & 27574.98 & & $\mathrm{~T}$ & 1.02 & \\
\hline 3706.255 & 20 & 26973.74 & $15587_{11 / 2}-42561_{9 / 2}^{\circ}$ & & & & 3624.198 & $200 r$ & 27584.45 & $15271_{15 / 2}-42855^{\circ}{ }_{15 / 2}$ & $\Delta \mathrm{J}=0$ & 1.10 & 0.33 \\
\hline 3704.358 & $50 h$ & 26987.55 & & $\mathrm{~T}$ & 1.41 & & 3622.355 & 10 & 27598.48 & $15587_{11 / 2}-43186_{11 / 2}^{\circ}$ & & & \\
\hline 3703.419 & 9 & 26994.39 & $19466_{13 / 2}-46461_{15 / 2}^{\circ}$ & & & & 3620.497 & $100 h$ & 27612.64 & & & & \\
\hline 3701.795 & $40 r$ & 27006.24 & $15587_{11 / 2}-42594_{9 / 2}^{\circ}$ & SI & 2.00 & 0.74 & 3619.545 & 4 & 27619.91 & & & & \\
\hline 3701.045 & $10 h$ & 27011.71 & & & & & 3617.040 & 10 & 27639.03 & $15271_{15 / 2}-42910^{\circ}{ }_{13 / 2}$ & & & \\
\hline 3696.593 & 5 & 27044.24 & $16957_{7 / 2}-44001_{9 / 2}^{\circ}$ & & & & 3616.398 & 2 & 27643.94 & & & & \\
\hline 3696.455 & $4 h$ & 27045.25 & & & & & 3616.355 & 20 & 27644.27 & $13119_{9 / 2}-40763^{\circ}{ }_{7 / 2}$ & SI & 1.72 & 0.41 \\
\hline 3695.385 & 3 & 27053.08 & & & & & 3615.650 & 4 & 27649.66 & & & & \\
\hline 3692.390 & 3 & 27075.02 & $17343_{\tau_{/ 2}}-44418_{7 / 2}^{\circ}$ & & & & 3614.820 & 20 & 27656.01 & $17752_{5 / 2}-45408^{\circ}{ }_{7 / 2}$ & & & \\
\hline 3691.580 & 5 & 27080.96 & & & & & 3613.565 & 3 & 27665.61 & & & & \\
\hline 3691.030 & $4 h$ & 27085.00 & & & & & 3613.200 & 3 & 27668.41 & & & & \\
\hline 3690.504 & 10 & 27088.86 & $13119_{9 / 2}-40208_{9 / 2}^{\circ}$ & & & & 3608.960 & 10 & 27700.91 & $15587_{11 / 2}-43288^{\circ}{ }_{13 / 2}$ & & & \\
\hline 3689.350 & $20 h$ & 27097.33 & & & & & 3608.604 & 2 & 27703.65 & $18990_{11 / 2}-46694^{\circ}{ }_{13 / 2}$ & & & \\
\hline 3688.731 & $10 h$ & 27101.88 & $19748_{9 / 2}-46850^{\circ}{ }_{11 / 2}$ & & & & 3607.261 & 20 & 27713.96 & $16742_{7 / 2}-44456^{\circ}{ }_{7 / 2}^{\circ / 2}$ & & & \\
\hline 3688.645 & 3 & 27102.51 & & & & & 3606.609 & $5 h$ & 27718.97 & & & & \\
\hline 3687.488 & 20 & 27111.02 & $17454_{13 / 2}-44565^{\circ}{ }_{15 / 2}$ & $\mathrm{~T}$ & 1.14 & & 3604.401 & 10 & 27735.95 & $13119_{9 / 2}-40855_{9 / 2}^{\circ}$ & & & \\
\hline 3687.248 & 10 & 27112.78 & & & & & 3603.872 & 10 & 27740.02 & & $\mathrm{~T}$ & 1.17 & \\
\hline 3684.771 & 20 & 27131.01 & $19466_{13 / 2}-46597^{\circ}{ }_{13 / 2}$ & $\Delta \mathrm{J}=0$ & 1.16 & 0.18 & 3603.661 & $30 h$ & 27741.64 & & & & \\
\hline 3684.458 & 10 & 27133.31 & $17613_{9 / 2}-44747^{\circ}{ }_{11 / 2}$ & & & & 3602.123 & $60 r$ & 27753.49 & $16456_{17 / 2}-44210^{\circ}{ }_{15 / 2}$ & SI & 1.22 & 0.15 \\
\hline 3684.145 & 6 & 27135.61 & $15271_{15 / 2}-42406^{\circ}{ }_{13 / 2}$ & & & & 3600.241 & 30 & 27768.00 & $18693_{15 / 2}-46461^{\circ}{ }_{15 / 2}$ & $\mathrm{~T}$ & 1.20 & \\
\hline 3684.059 & 5 & 27136.25 & & & & & 3599.188 & 5 & 27776.12 & & & & \\
\hline 3683.475 & 10 & 27140.55 & & & & & 3598.616 & $200 r$ & 27780.54 & $1527 l_{15 / 2}-43051^{\circ}{ }_{15 / 2}$ & $\Delta \mathrm{J}=0$ & 1.15 & 0.84 \\
\hline 3681.194 & 20 & 27157.37 & $16456_{17 / 2}-43614^{\circ}{ }_{15 / 2}$ & SI & 1.48 & 0.40 & 3596.487 & 20 & 27796.98 & & $\mathrm{~T}$ & 0.85 & \\
\hline 3679.113 & $3 h$ & 27172.73 & & & & & 3595.600 & 4 & 27803.84 & & & & \\
\hline 3677.728 & $40 r$ & 27182.96 & $15587_{11 / 2}-42770_{9 / 2}^{\circ}$ & SI & 1.44 & 0.18 & 3594.404 & 30 & 27813.09 & & $\mathrm{~T}$ & 1.09 & \\
\hline 3677.004 & $4 h$ & 27188.31 & & & & & 3593.867 & 20 & 27817.24 & $16957_{7 / 2}-44774_{9 / 2}^{\circ}$ & & & \\
\hline
\end{tabular}


APPENDIX - Spectral Lines of Neutral Thulium-Continued

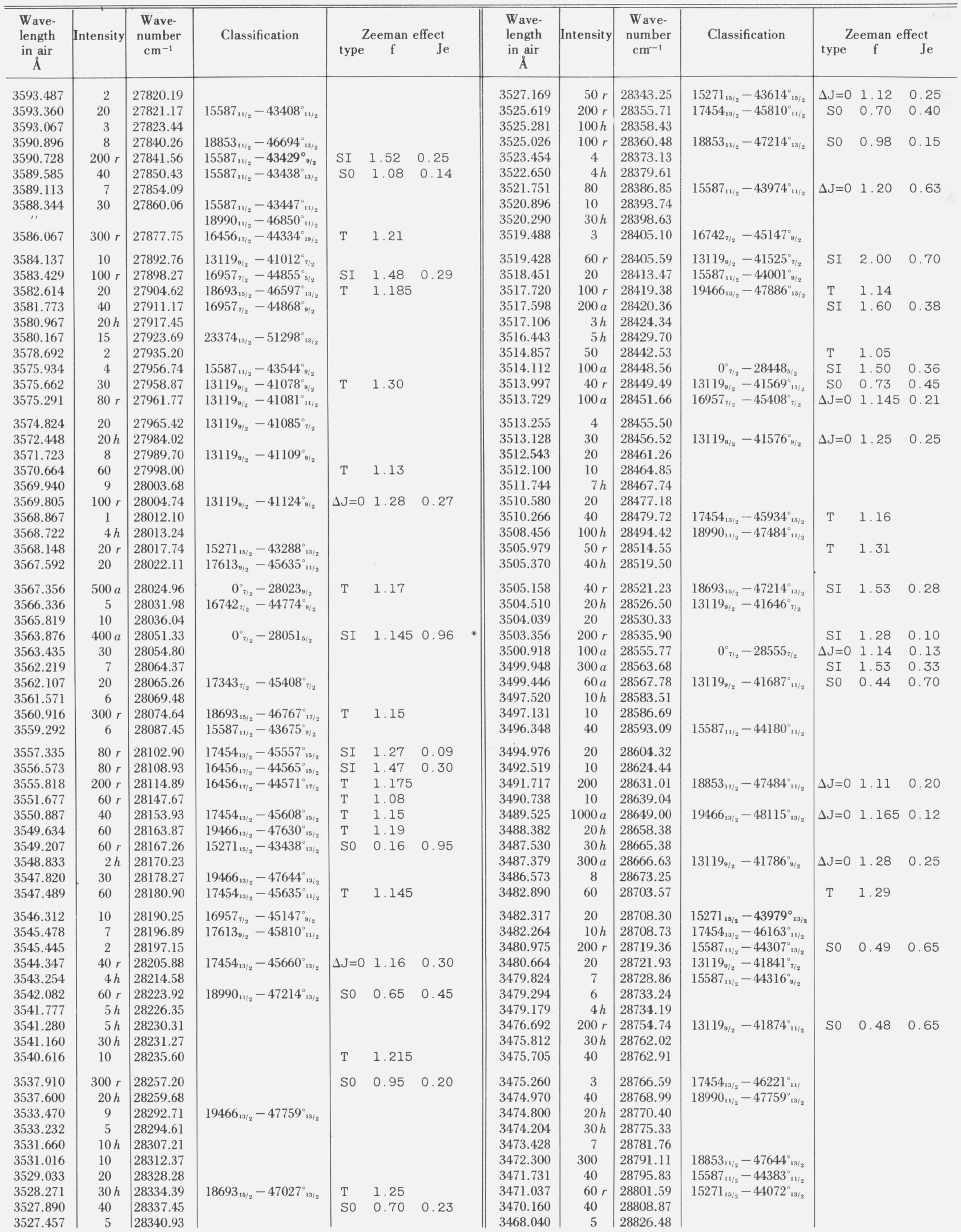


APPENDIX - Spectral Lines of Neutral Thulium - Continued

\begin{tabular}{|c|c|c|c|c|c|c|c|c|c|c|c|c|c|c|}
\hline \multirow{2}{*}{$\begin{array}{c}\text { Wave- } \\
\text { length } \\
\text { in air } \\
\AA\end{array}$} & \multirow[t]{2}{*}{ Intensity } & \multirow{2}{*}{$\begin{array}{c}\text { Wave- } \\
\text { number } \\
\mathrm{cm}^{-1}\end{array}$} & \multirow[t]{2}{*}{ Classification } & \multirow{2}{*}{\multicolumn{4}{|c|}{ Zeeman effect }} & $\begin{array}{l}\text { Wave- } \\
\text { length }\end{array}$ & Intensity & $\begin{array}{l}\text { Wave- } \\
\text { number }\end{array}$ & Classification & & eeman & effect \\
\hline & & & & & & & & in air & & $\mathrm{cm}^{-1}$ & & type & f & Je \\
\hline 3467.513 & $200 r$ & 28830.86 & $15587_{11 / 2}-44418^{\circ}{ }_{13 / 2}$ & so & 0.53 & 0.605 & & 3395.196 & 3 & 29444.93 & & & & \\
\hline 3465.720 & $40 h$ & 28845.77 & & & & & & 3394.185 & $200 h$ & 29453.70 & & & & \\
\hline 3462.634 & 6 & 28871.48 & & & & & & 3393.980 & 70 & 29455.48 & & & & \\
\hline 3458.551 & $40 r$ & 28905.56 & $18853_{11 / 2}-47759^{\circ}{ }_{13 / 2}$ & $\mathrm{~T}$ & 1.17 & & & 3393.186 & $1000 r$ & 29462.37 & $13119_{9 / 2}-42581^{\circ}{ }_{11 / 2}$ & so & 0.37 & 0.75 \\
\hline 3458.457 & 20 & 28906.35 & & & & & & 3392.693 & 20 & 29466.65 & $8771^{\circ}{ }^{\circ / 2}-38237_{3 / 2}$ & & & \\
\hline 3456.358 & $100 a$ & 28923.90 & & $\mathrm{~T}$ & 1.135 & & & 3392.391 & 6 & 29469.28 & $21997_{11 / 2}-51466^{\circ}{ }_{13 / 2}$ & & & \\
\hline 3454.738 & $100 a$ & 28937.47 & $18693_{15 / 2}-47630^{\circ}{ }_{15 / 2}$ & $\Delta \mathrm{J}=0$ & 1.16 & 0.15 & & 3392.241 & 100 & 29470.58 & $15587_{11 / 2}-45058^{\circ}{ }_{11 / 2}$ & & & \\
\hline 3454.505 & $50 a$ & 28939.42 & $15271_{15 / 2}-44210^{\circ}{ }_{15 / 2}$ & $\Delta \mathrm{J}=0$ & 1.12 & 0.50 & & 3392.029 & 5 & 29472.42 & & & & \\
\hline 3453.548 & 4 & 28947.44 & & & & & & 3391.865 & 8 & 29473.85 & & & & \\
\hline 3453.027 & $100 r$ & 28951.80 & $18693_{15 / 2}-47644_{13 / 2}^{\circ}$ & $\mathrm{T}$ & 1.18 & & & 3391.800 & 100 & 29474.41 & $13119_{9 / 2}-42594_{9 / 2}^{\circ}$ & & & \\
\hline 3452.020 & $4 h$ & 28960.25 & & & & & & 3391.430 & 200 & 29477.63 & $16456_{17 / 2}-45934^{\circ}{ }_{15 / 2}$ & SI & 1.26 & 0.08 \\
\hline 3450.370 & $4 h$ & 28974.10 & & & & & & 3391.262 & $8 h$ & 29479.09 & & & & \\
\hline 3448.801 & $4 h$ & 28987.28 & & & & & & 3390.836 & 200 & 29482.79 & $15587_{11 / 2}-45070_{9 / 2}^{\circ}$ & & & \\
\hline 3447.353 & $100 r$ & 28999.45 & $13119_{9 / 2}-42119_{11 / 2}^{\circ}$ & So & 0.20 & 0.92 & & 3390.365 & 5 & 29486.88 & & & & \\
\hline 3446.893 & 7 & 29003.32 & $15587_{11 / 2}-44591^{\circ}{ }_{13 / 2}$ & & & & & 3390.245 & 100 & 29487.93 & & & & \\
\hline 3446.546 & $100 a$ & 29006.24 & $17454_{13 / 2}-46461_{15 / 2}^{\circ}$ & SI & 1.40 & 0.20 & & 3389.461 & 9 & 29494.75 & & & & \\
\hline 3445.668 & $50 a$ & 29013.63 & $13119_{9 / 2}-42133_{7 / 2}^{\circ / 2}$ & & & & & 3389.339 & 10 & 29495.81 & & & & \\
\hline 3444.162 & $100 a$ & 29026.32 & & $\mathrm{~T}$ & 1.19 & & & 3385.084 & $2000 r$ & 29532.89 & $15271_{15 / 2}-44803^{\circ}{ }_{13 / 2}$ & So & 0.92 & 0.15 \\
\hline 3442.992 & $100 a$ & 29036.18 & $15271_{15 / 2}-44307^{\circ}{ }_{13 / 2}$ & So & 0.72 & 0.33 & & 3383.419 & 10 & 29547.42 & $8771_{5 / 2}^{\circ / 2}-38318_{3 / 2}$ & & & \\
\hline 3440.443 & 2 & 29057.70 & & & & & & 3383.268 & $4 h$ & 29548.74 & & & & \\
\hline 3439.425 & 10 & 29066.30 & $18693_{15 / 2}-47759^{\circ}{ }_{13 / 2}$ & & & & & 3382.038 & 100 & 29559.48 & $15587_{11 / 2}-45147_{9 / 2}^{\circ}$ & & & \\
\hline 3436.599 & 40 & 29090.20 & & $\mathrm{~T}$ & 1.13 & & & 3381.499 & $3 h$ & 29564.19 & & & & \\
\hline 3435.348 & $100 a$ & 29100.79 & $16456_{17 / 2}-45557^{\circ}{ }_{15 / 2}$ & SI & 1.25 & 0.07 & & 3381.390 & $50 h$ & 29565.15 & & & & \\
\hline 3433.409 & $4 h$ & 29117.22 & & & & & & 3380.688 & $1000 a$ & 29571.29 & $8771^{\circ}{ }_{s / 2}-38342_{7 / 2}$ & SI & 0.85 & 1.108 \\
\hline 3430.385 & $20 r$ & 29142.89 & $17454_{13 / 2}-46597_{13 / 2}^{\circ}$ & & & & & 3380.534 & $2000 a$ & 29572.63 & $17454_{13 / 2}-47027^{\circ}{ }_{13 / 2}$ & $\Delta \mathrm{J}=0$ & 1.16 & 0.13 \\
\hline 3429.828 & $20 r$ & 29147.62 & $15271_{15 / 2}-44418^{\circ}{ }_{13 / 2}^{\circ / 2}$ & & & & & 3379.915 & 1 & 29578.05 & & & & \\
\hline 3429.332 & $200 a$ & 29151.84 & $16456_{17 / 2}-45608^{\circ}{ }_{15 / 2}^{\circ / 2}$ & & & & & 3377.392 & 3 & 29600.14 & & & & \\
\hline 3428.471 & $200 a$ & 29159.16 & $15587_{11 / 2}-44747^{\circ}{ }_{11 / 2}$ & $\Delta \mathrm{J}=0$ & 1.22 & 0.45 & & 3376.963 & $3 h$ & 29603.90 & & & & \\
\hline 3426.678 & 100 & 29174.42 & & & & & & 3375.890 & 10 & 29613.31 & & & & \\
\hline 3425.262 & 15 & 29186.48 & $15587_{11 / 2}-44774_{9 / 2}^{\circ}$ & & & & & 3374.970 & 20 & 29621.39 & & & & \\
\hline 3424.899 & 60 & 29189.57 & & & & & & 3374.853 & 100 & 29622.41 & $13119_{9 / 2}-42742^{\circ}{ }_{\gamma / 2}$ & & & \\
\hline 3424.498 & $400 r$ & 29192.99 & $18693_{15 / 2}-47886_{15 / 2}^{\circ}$ & $\Delta \mathrm{J}=0$ & 1.18 & 0.25 & & 3374.163 & 2 & 29628.47 & & & & \\
\hline 3423.984 & 40 & 29197.37 & & & & & & 3373.482 & 3 & 29634.45 & & & & \\
\hline 3422.559 & 100 & 29209.53 & & & & & & 3372.750 & 30 & 29640.88 & & & & \\
\hline 3421.791 & $700 r$ & 29216.08 & $15587_{11 / 2}-44803^{\circ}{ }_{13 / 2}$ & so & 0.28 & 0.80 & & 3371.576 & 100 & 29651.20 & $13119_{9 / 2}-42770_{9 / 2}^{\circ}$ & & & \\
\hline 3421.697 & 100 & 29216.89 & & & & & & 3369.321 & $3 h$ & 29671.05 & & & & \\
\hline 3421.202 & 20 & 29221.11 & & & & & & 3368.597 & $2000 a$ & 29677.42 & & $\mathrm{~T}$ & 1.04 & 0.08 \\
\hline 3420.203 & 50 & 29229.65 & & & & & & 3368.186 & 3 & 29681.05 & & & & \\
\hline 3420.146 & 100 & 29230.14 & & & & & & 3368.010 & 4 & 29682.60 & & & & \\
\hline 3419.644 & 8 & 29234.43 & & & & & & 3367.805 & 1 & 29684.40 & & & & \\
\hline 3419.359 & 100 & 29236.86 & & & & & & 3367.570 & 3 & 29686.47 & & & & \\
\hline 3419.071 & 100 & 29239.33 & $17454_{13 / 2}-46694_{13 / 2}^{\circ}$ & & & & & 3366.676 & 2 & 29694.36 & & & & \\
\hline 3418.625 & 200 & 29243.14 & $8771_{5 / 2}^{\circ}-38014_{5 / 2}$ & $\Delta \mathrm{J}=0$ & 0.85 & 1.105 & * & 3365.900 & $1000 h$ & 29701.20 & $15587_{11 / 2}-45289^{\circ}{ }_{11 / 2}$ & $\Delta \mathrm{J}=0$ & 1.12 & 0.55 \\
\hline 3417.692 & 300 & 29251.12 & $13119_{9 / 2}-42370^{\circ}{ }_{7 / 2}$ & & & & & 3365.327 & 3 & 29706.26 & & & & \\
\hline 3417.416 & 10 & 29253.48 & & & & & & 3363.781 & 200 & 29719.91 & $13119_{9 / 2}-42839_{9 / 2}^{\circ}$ & & & \\
\hline 3416.588 & $3000 a$ & 29260.57 & $0_{7 / 2}^{\circ}-29260_{7 / 2}$ & $\Delta \mathrm{J}=0$ & 1.143 & 0.928 & * & 3363.535 & 100 & 29722.09 & & & & \\
\hline 3416.430 & 10 & 29261.93 & $18853_{11 / 2}-48115^{\circ}{ }_{13 / 2}$ & & & & & 3363.465 & 10 & 29722.70 & & & & \\
\hline 3416.146 & 4 & 29264.36 & & & & & & 3359.549 & 2 & 29757.35 & & & & \\
\hline 3414.521 & $50 h$ & 29278.29 & & & & & & 3359.337 & $50 h$ & 29759.23 & $21997_{11 / 2}-51756^{\circ}{ }_{11 / 2}$ & & & \\
\hline 3414.273 & 20 & 29280.41 & $15587_{11 / 2}-44868_{9 / 2}^{\circ}$ & & & & & 3358.601 & 2 & 29765.75 & & & & \\
\hline 3413.989 & $200 h$ & 29282.85 & & & & & & 3358.265 & 20 & 29768.73 & & & & \\
\hline 3413.330 & 2 & 29288.50 & & & & & & 3358.057 & 4 & 29770.57 & & & & \\
\hline 3413.035 & 200 & 29291.03 & & & & & & 3356.161 & 10 & 29787.39 & & & & \\
\hline 3412.590 & $3000 a$ & 29294.85 & $15271_{15 / 2}-44565_{15 / 2}^{\circ}$ & $\Delta \mathrm{J}=0$ & 1.11 & 0.41 & & 3354.600 & $100 r$ & 29801.25 & $15587_{11 / 2}-45389^{\circ}{ }_{13 / 2}$ & & & \\
\hline 3412.404 & 3 & 29296.45 & & & & & & 3354.167 & 1 & 29805.10 & & & & \\
\hline 3411.893 & 200 & 29300.84 & $15271_{15 / 2}-44571^{\circ}{ }_{17 / 2}$ & & & & & 3353.648 & 10 & 29809.71 & & & & \\
\hline "' & & & $21997_{11 / 2}-51298^{\circ}{ }_{13 / 2}$ & & & & & 3352.923 & 5 & 29816.15 & & & & \\
\hline 3410.048 & $1000 a$ & 29316.69 & $0_{7 / 2}^{\circ}-29316_{9 / 2}$ & So & 0.40 & 0.58 & & 3351.530 & 10 & 29828.55 & & & & \\
\hline 3409.650 & 100 & 29320.11 & $15271_{15 / 2}-44591^{\circ}{ }_{13 / 2}$ & & & & & 3351.390 & 8 & 29829.79 & & & & \\
\hline 3408.747 & 40 & 29327.88 & & & & & & 3351.224 & 10 & 29831.27 & & & & \\
\hline 3405.967 & $2000 a$ & 29351.82 & $8771_{s / 2}^{\circ}-38123_{7 / 2}$ & SI & 0.83 & 1.15 & * & 3349.987 & 1000 & 29842.28 & $13119_{9 / 2}-42961^{\circ}{ }_{11 / 2}$ & so & 1.30 & 1.195 \\
\hline 3402.694 & 3 & 29380.05 & & & & & & 3349.808 & 60 & 29843.88 & & & & \\
\hline 3401.751 & $40 h$ & 29388.19 & & & & & & 3348.766 & 100 & 29853.16 & & & & \\
\hline 3400.887 & $200 h$ & 29395.66 & $17454_{13 / 2}-46850^{\circ}{ }_{11 / 2}$ & $\mathrm{~T}$ & 1.14 & & & 3346.676 & 1 & 29871.81 & & & & \\
\hline 3399.782 & 200 & 29405.21 & & & & & & 3346.033 & 2 & 29877.55 & & & & \\
\hline 3399.593 & 3 & 29406.85 & & & & & & 3344.163 & 8 & 29894.25 & & & & \\
\hline 3398.544 & 10 & 29415.92 & & & & & & 3343.737 & 200 & 29898.06 & $15587_{11 / 2}-45485^{\circ}{ }_{11 / 2}$ & & & \\
\hline 3397.867 & $300 r$ & 29421.78 & $13119_{9 / 2}-42541_{11 / 2}^{\circ}$ & so & 1.31 & 1.10 & * & 3342.896 & 9 & 29905.58 & & & & \\
\hline 3397.773 & 200 & 29422.60 & $18693_{15 / 2}-48115_{13 / 2}^{\circ}$ & & & & & 3342.390 & 20 & 29910.11 & & & & \\
\hline 3396.724 & 8 & 29431.68 & & & & & & 3342.324 & 30 & 29910.70 & $13119_{9 / 2}-43030_{9 / 2}^{\circ}$ & & & \\
\hline
\end{tabular}


APPENDIX - Spectral Lines of Neutral Thulium-Continued

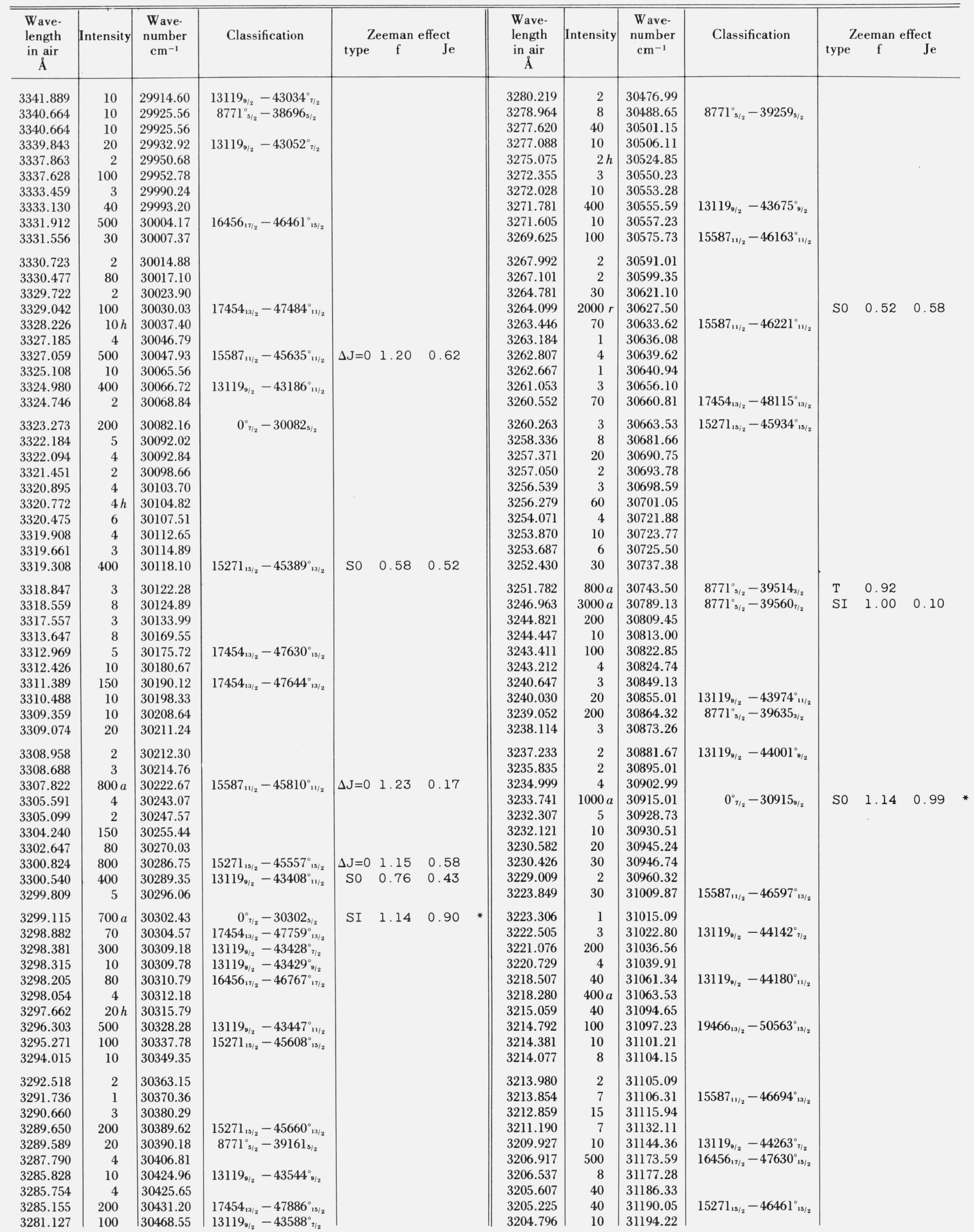


APPENDIX - Spectral Lines of Neutral Thulium-Continued

\begin{tabular}{|c|c|c|c|c|c|c|c|c|c|c|c|c|c|c|}
\hline \multirow{2}{*}{$\begin{array}{c}\text { Wave- } \\
\text { length } \\
\text { in air } \\
\AA\end{array}$} & \multirow[t]{2}{*}{ Intensity } & \multirow{2}{*}{$\begin{array}{l}\text { Wave- } \\
\text { number } \\
\mathrm{cm}^{-1}\end{array}$} & \multirow[t]{2}{*}{ Classification } & \multicolumn{3}{|c|}{ Zeeman effect } & \multirow{2}{*}{$\begin{array}{l}\text { Wave- } \\
\text { length } \\
\text { in air } \\
\AA\end{array}$} & Intensity & $\begin{array}{l}\text { Wave- } \\
\text { number }\end{array}$ & Classification & & Ceman $e$ & ffect & \\
\hline & & & & type & & & & & $\mathrm{cm}^{-1}$ & & type & $\mathrm{f}$ & & \\
\hline 3204.502 & 50 & 31197.08 & $13119_{9 / 2}-44316_{9 / 2}^{\circ}$ & & & & 3131.686 & 10 & 31922.43 & $8771_{5 / 2}^{\circ}-40693_{3 / 2}$ & & & & \\
\hline 3201.645 & 300 & 31224.92 & $8771_{5 / 2}^{\circ}-39996_{7 / 2}$ & & & & 3130.083 & 50 & 31938.78 & $13119_{9 / 2}-45058^{\circ}{ }_{11 / 2}$ & & & & \\
\hline 3201.255 & 20 & 31228.72 & & & & & 3129.638 & 20 & 31943.32 & $15271_{15 / 2}-47214^{\circ}{ }_{13 / 2}$ & & & & \\
\hline 3200.677 & 5 & 31234.36 & & & & & 3128.891 & 20 & 31950.95 & $13119_{9 / 2}-45070_{9 / 2}^{\circ}$ & & & & \\
\hline 3197.640 & 15 & 31264.03 & $13119_{9 / 2}-44383_{11 / 2}^{\circ}$ & & & & 3125.506 & 2 & 31985.55 & & & & & \\
\hline 3195.685 & 30 & 31283.15 & & & & & 3124.087 & 100 & 32000.07 & $19466_{13 / 2}-51466^{\circ}{ }_{13 / 2}$ & & & & \\
\hline 3194.592 & 150 & 31293.86 & & & & & 3123.907 & 30 & 32001.92 & & & & & \\
\hline 3194.431 & 20 & 31295.43 & $8771^{\circ}{ }_{s / 2}-40066_{s / 2}$ & & & & 3122.959 & 1 & 32011.63 & & & & & \\
\hline 3194.085 & 150 & 31298.82 & $13119_{9 / 2}-44418^{\circ}{ }_{7 / 2}$ & & & & 3122.533 & $3000 r$ & 32016.00 & $8771_{s / 2}^{\circ}-40787_{s / 2}$ & $\Delta \mathrm{J}=0$ & 0.88 & 0.14 & \\
\hline 3193.778 & 2 & 31301.83 & & & & & 3121.390 & 100 & 32027.72 & $13119_{9 / 2}-45147_{9 / 2}^{\circ}$ & & & & \\
\hline 3192.582 & 8 & 31313.56 & & & & & 3121.038 & 8 & 32031.34 & $8771_{s / 2}^{\circ}-40802_{7 / 2}$ & & & & \\
\hline 3192.548 & 10 & 31313.89 & & & & & 3120.881 & 7 & 32032.95 & & & & & \\
\hline 3191.234 & 400 & 31326.78 & & & & & 3119.522 & 10 & 32046.90 & & & & & \\
\hline 3190.228 & 40 & 31336.66 & $13119_{9 / 2}-44456_{7 / 2}^{\circ}$ & & & & 3118.523 & 50 & 32057.17 & $15587_{11 / 2}-47644^{\circ}{ }_{13 / 2}$ & & & & \\
\hline 3189.150 & 40 & 31347.25 & & & & & 3116.348 & 4 & 32079.54 & & & & & \\
\hline 3188.306 & 3 & 31355.55 & & & & & 3116.261 & 30 & 32080.44 & & & & & \\
\hline 3186.058 & 150 & 31377.68 & & & & & 3113.848 & $2 h$ & 32105.29 & & & & & \\
\hline 3183.555 & 2 & 31402.34 & & & & & 3113.507 & 2 & 32108.81 & & & & & \\
\hline 3183.416 & 2 & 31403.72 & & & & & 3110.015 & 3 & 32144.86 & & & & & \\
\hline 3182.148 & 4 & 31416.23 & & & & & 3108.358 & 1 & 32162.00 & & & & & \\
\hline 3181.923 & 3 & 31418.45 & & & & & 3108.293 & 3 & 32162.67 & & & & & \\
\hline 3181.453 & 40 & 31423.09 & $15271_{15 / 2}-46694^{\circ}{ }_{13 / 2}$ & & & & 3107.932 & 5 & 32166.40 & & & & & \\
\hline 3180.947 & 3 & 31428.09 & & & & & 3107.642 & $30 h$ & 32169.41 & $13119_{9 / 2}-45289^{\circ}{ }_{11 / 2}$ & & & & \\
\hline 3180.837 & 20 & 31429.18 & $16456_{17 / 2}-47886^{\circ}{ }_{15 / 2}$ & & & & 3107.152 & $500 a$ & 32174.48 & $0_{7 / 2}^{\circ}-32174_{s / 2}^{\circ}$ & $\mathrm{T}$ & 1.10 & 0.05 & \\
\hline 3180.565 & $1000 a$ & 31431.86 & $0_{7 / 2}^{\circ}-31431_{5 / 2}$ & SI & 1.31 & 0.17 & 3105.741 & 2 & 32189.10 & & & & & \\
\hline 3180.195 & 20 & 31435.52 & & & & & 3104.969 & 2 & 32197.10 & & & & & \\
\hline 3180.030 & 150 & 31437.15 & & & & & 3104.888 & 5 & 32197.94 & & & & & \\
\hline 3179.780 & 30 & 31439.62 & $15587_{11 / 2}-47027^{\circ}{ }_{13 / 2}$ & & & & 3103.826 & 10 & 32208.96 & & & & & \\
\hline 3179.687 & $300 a$ & 31440.54 & $0_{7 / 2}^{\circ}-31440_{9 / 2}$ & $\mathrm{~T}$ & 1.13 & & 3103.698 & 5 & 32210.28 & & & & & \\
\hline 3179.044 & 5 & 31446.90 & & & & & 3103.646 & 40 & 32210.82 & & & & & \\
\hline 3178.241 & 10 & 31454.85 & & & & & 3098.222 & 4 & 32267.21 & & & & & \\
\hline 3176.168 & 1 & 31475.38 & & & & & 3096.032 & 300 & 32290.04 & $19466_{13 / 2}-51756_{11 / 2}^{\circ}$ & & & & \\
\hline 3175.724 & 3 & 31479.78 & & & & & 3092.033 & 20 & 32331.80 & & & & & \\
\hline 3174.018 & 8 & 31496.70 & $15271_{15 / 2}-46767^{\circ}{ }_{17 / 2}$ & & & & 3090.471 & 40 & 32348.14 & $13119_{9 / 2}-45467_{9 / 2}^{\circ}$ & & & & \\
\hline 3173.484 & 20 & 31502.00 & & & & & 3089.522 & 30 & 32358.07 & & & & & \\
\hline 3173.428 & 10 & 31502.55 & & & & & 3088.737 & 4 & 32366.30 & $13119_{9 / 2}-45485^{\circ}{ }_{11 / 2}$ & & & & \\
\hline 3172.654 & $3000 a$ & 31510.24 & $0^{\circ}{ }_{7 / 2}-31510_{\gamma^{2} / 2}$ & $\mathrm{~T}$ & 1.10 & 0.11 & 3088.464 & 1 & 32369.16 & & & & & \\
\hline 3172.036 & 1 & 31516.37 & & & & & 3087.936 & 5 & 32374.69 & & & & & \\
\hline 3171.277 & 10 & 31523.92 & & & & & 3086.854 & 2 & 32386.04 & & & & & \\
\hline 3168.107 & 10 & 31555.46 & & & & & 3084.783 & $400 a$ & 32407.78 & $0_{7 / 2}^{\circ}-32407_{9 / 2}$ & & & & \\
\hline 3166.572 & 60 & 31570.76 & & & & & 3081.388 & 3 & 32443.48 & & & & & \\
\hline 3164.731 & 5 & 31589.12 & $8771_{s / 2}^{\circ}-40360_{z^{\prime} / 2}$ & & & & 3081.121 & $2000 a$ & 32446.30 & $0_{\tau^{2} / 2}^{\circ}-32446_{7 / 2}$ & $\Delta \mathrm{J}=0$ & 1.143 & 0.89 & * \\
\hline 3162.693 & $7 h$ & 31609.47 & & & & & 3080.212 & 4 & 32455.87 & & & & & \\
\hline 3162.612 & 5 & 31610.28 & & & & & 3080.083 & 20 & 32457.23 & & & & & \\
\hline 3161.200 & 4 & 31624.40 & & & & & 3079.034 & 2 & 32468.29 & & & & & \\
\hline 3160.989 & 2 & 31626.51 & $15587_{11 / 2}-47214^{\circ}{ }_{13 / 2}$ & & & & 3078.822 & 4 & 32470.52 & & & & & \\
\hline 3160.895 & 100 & 31627.45 & $13119_{9 / 2}-44747_{11 / 2}^{\circ}$ & & & & 3078.107 & 300 & 32478.07 & & & & & \\
\hline 3159.731 & 6 & 31639.11 & & & & & 3077.717 & 8 & 32482.18 & & & & & \\
\hline 3158.177 & 10 & 31654.67 & $13119_{9 / 2}-44774_{9 / 2}^{\circ}$ & & & & 3074.501 & 80 & 32516.16 & $13119_{9 / 2}-45635_{11 / 2}^{\circ}$ & & & & \\
\hline 3157.990 & 5 & 31656.55 & & & & & 3073.391 & 10 & 32527.90 & $15587_{11 / 2}-48115_{13 / 2}^{\circ}$ & & & & \\
\hline 3156.688 & 3 & 31669.60 & & & & & 3072.063 & 4 & 32541.96 & & & & & \\
\hline 3154.766 & 2 & 31688.90 & & & & & 3071.839 & 5 & 32544.33 & & & & & \\
\hline 3154.297 & 3 & 31693.61 & & & & & 3068.904 & 15 & 32575.46 & & & & & \\
\hline 3153.223 & 150 & 31704.40 & & & & & 3067.728 & 2 & 32587.94 & & & & & \\
\hline 3148.611 & 1 & 31750.84 & & & & & 3066.096 & 200 & 32605.29 & $18693_{15 / 2}-51298^{\circ}{ }_{13 / 2}$ & & & & \\
\hline 3148.058 & 400 & 31756.42 & $15271_{15 / 2}-47027^{\circ}{ }_{13 / 2}$ & & & & 3065.382 & 10 & 32612.88 & $18853_{11 / 2}-51466^{\circ}{ }_{13 / 2}$ & & & & \\
\hline 3146.574 & 400 & 31771.40 & & & & & 3063.584 & 3 & 32632.02 & & & & & \\
\hline 3145.637 & 20 & 31780.86 & & & & & 3060.440 & 20 & 32665.54 & & & & & \\
\hline 3144.105 & 20 & 31796.34 & & & & & 3060.176 & 1 & 32668.36 & & & & & \\
\hline 3142.838 & 2 & 31809.16 & & & & & 3058.065 & 400 & 32690.91 & $13119_{9 / 2}-45810^{\circ}{ }_{11 / 2}$ & & & & \\
\hline 3142.433 & 400 & 31813.26 & $8771_{s / 2}^{\circ}-40584_{s / 2}$ & & & & 3055.636 & 10 & 32716.90 & & & & & \\
\hline 3140.611 & 100 & 31831.72 & $19466_{13 / 2}-51298^{\circ}{ }_{13 / 2}$ & & & & 3055.179 & 70 & 32721.79 & $8771_{s_{5 / 2}}^{\circ}-41493_{s_{5 / 2}}$ & & & & \\
\hline 3139.795 & 10 & 31839.99 & & & & & 3051.033 & $50 h$ & 32766.26 & $18990_{11 / 2}^{/ 2}-51756_{11 / 2}^{\circ}$ & & & & \\
\hline 3137.010 & 30 & 31868.25 & & & & & 3050.367 & 40 & 32773.41 & $8771_{s^{\prime} / 2}^{\circ / 2}-41544_{s / 2}$ & & & & \\
\hline 3136.755 & 500 & 31870.85 & $18693_{15 / 2}-50563^{\circ}{ }_{15 / 2}$ & $\mathrm{~T}$ & 1.18 & & 3050.339 & 30 & 32773.71 & $18693_{1 \mathrm{~s} / 2}-51466^{\circ}{ }_{13 / 2}$ & & & & \\
\hline 3136.539 & 20 & 31873.04 & & & & & 3049.985 & 2 & 32777.51 & & & & & \\
\hline 3136.216 & 20 & 31876.32 & & & & & 3049.090 & 2 & 32787.13 & & & & & \\
\hline 3136.031 & 5 & 31878.20 & $8771_{s / 2}^{\circ}-40649_{7 / 2}$ & & & & 3046.870 & $3000 a$ & 32811.02 & $0_{7 / 2}^{\circ}-32811_{7 / 2}$ & $\Delta \mathrm{J}=0$ & 1.143 & 1.04 & 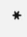 \\
\hline 3134.177 & 40 & 31897.06 & $15587_{11 / 2}-47484_{11 / 2}^{\circ}$ & & & & 3044.469 & $30 h$ & 32836.90 & & & & & \\
\hline 3133.239 & 10 & 31906.61 & & & & & 3039.631 & 20 & 32889.16 & & & & & \\
\hline
\end{tabular}


APPENDiX - Spectral Lines of Neutral Thulium - Continued

\begin{tabular}{|c|c|c|c|c|c|c|c|c|c|c|c|c|c|c|}
\hline \multirow{2}{*}{$\begin{array}{c}\text { Wave- } \\
\text { length } \\
\text { in air } \\
\AA\end{array}$} & \multirow[t]{2}{*}{ Intensity } & \multirow{2}{*}{$\begin{array}{l}\text { Wave- } \\
\text { number } \\
\mathrm{cm}^{-1}\end{array}$} & \multirow[t]{2}{*}{ Classification } & \multirow{2}{*}{\multicolumn{4}{|c|}{$\begin{array}{l}\text { Zeeman effect } \\
\text { e f } \quad \text { Je }\end{array}$}} & \multirow{2}{*}{$\begin{array}{c}\text { Wave- } \\
\text { length } \\
\text { in air } \\
\AA\end{array}$} & Intensity & $\begin{array}{l}\text { Wave- } \\
\text { number }\end{array}$ & Classification & & eeman $\mathrm{e}$ & ffect \\
\hline & & & & & & & & & & & & type & $\mathrm{f}$ & \\
\hline 3039.067 & 20 & 32895.26 & & & & & & 2926.431 & 40 & 34161.32 & & & & \\
\hline 3038.370 & $200 h$ & 32902.81 & $18853_{11 / 2}-51756^{\circ}{ }_{11 / 2}$ & & & & & 2925.842 & $3 h$ & 34168.20 & & & & \\
\hline 3036.848 & $80 h$ & 32919.30 & $18837_{9 / 2}-51756^{\circ}{ }_{11 / 2}$ & & & & & 2925.049 & 1 & 34177.46 & & & & \\
\hline 3035.784 & 2 & 32930.84 & & & & & & 2924.297 & 2 & 34186.25 & $8771_{s / 2}^{\circ}-42957_{5 / 2}$ & & & \\
\hline 3034.642 & 3 & 32943.23 & & & & & & 2921.958 & 3 & 34213.61 & & & & \\
\hline 3032.613 & 30 & 32965.27 & & & & & & 2921.706 & 10 & 34216.56 & & & & \\
\hline 3031.280 & 40 & 32979.76 & $8771_{s / 2}^{\circ}-41751_{5 / 2}$ & & & & & 2920.758 & 1 & 34227.67 & & & & \\
\hline 3030.567 & 20 & 32987.52 & & & & & & 2917.411 & 2 & 34266.93 & & & & \\
\hline 3030.491 & 10 & 32988.35 & & & & & & 2916.372 & 4 & 34.279 .14 & & & & \\
\hline 3029.061 & 2 & 33003.92 & & & & & & 2915.985 & 1 & 34283.69 & & & & \\
\hline 3026.694 & 2 & 33029.73 & & & & & & 2915.879 & 20 & 34284.94 & $8771_{s / 2}^{\circ}-43056_{s / 2}$ & & & \\
\hline 3025.449 & 10 & 33043.32 & & & & & & 2915.458 & $5 h$ & 34289.89 & & & & \\
\hline 3025.386 & 40 & 33044.01 & $13119_{9 / 2}-46163^{\circ}{ }_{11 / 2}$ & & & & & 2914.839 & $1000 a$ & 34297.17 & $0_{7 / 2}^{\circ}-34297_{7 / 2}$ & $\Delta \mathrm{J}=0$ & 1.143 & 0.915 \\
\hline 3024.498 & 8 & 33053.71 & & & & & & 2914.665 & 2 & 34299.22 & & & & \\
\hline 3023.485 & 20 & 33064.79 & $8771_{s / 2}^{\circ}-41836_{5 / 2}$ & & & & & 2914.442 & $30 h$ & 34301.84 & $17454_{13 / 2}-51756^{\circ}{ }_{11 / 2}$ & & & \\
\hline 3020.101 & 10 & 33101.83 & $13119_{9 / 2}-46221^{\circ}{ }_{11 / 2}$ & & & & & 2912.953 & 20 & 34319.37 & & & & \\
\hline 3019.436 & 70 & 33109.12 & $17454_{13 / 2}-50563^{\circ}{ }_{15 / 2}$ & & & & & 2912.342 & 1 & 34326.57 & & & & \\
\hline 3016.834 & 2 & 33137.68 & & & & & & 2909.301 & 5 & 34362.45 & & & & \\
\hline 3013.639 & 10 & 33172.81 & & & & & & 2908.502 & 2 & 34371.89 & & & & \\
\hline 3009.480 & 10 & 33218.65 & & & & & & 2908.161 & 10 & 34375.92 & & & & \\
\hline 3008.446 & 200 & 33230.07 & & & & & & 2907.031 & 5 & 34389.28 & $8771_{5 / 2}^{\circ}-43160_{3 / 2}$ & & & \\
\hline 3007.281 & 3 & 33242.94 & & & & & & 2906.270 & 3 & 34398.29 & & & & \\
\hline 3007.118 & 1 & 33244.74 & & & & & & 2904.065 & 2 & 34.424 .41 & & & & \\
\hline 3005.816 & 10 & 33259.14 & & & & & & 2902.226 & 10 & 34446.22 & & & & \\
\hline 3002.396 & 7 & 33297.03 & & & & & & 2901.864 & 2 & 34450.51 & & & & \\
\hline 3001.334 & 2 & 33308.81 & & & & & & 2900.088 & 50 & 34471.61 & & & & \\
\hline 2999.448 & 5 & 33329.75 & & & & & & 2897.580 & $20 h$ & 34501.45 & & & & \\
\hline 2995.079 & 30 & 33378.37 & & & & & & 2894.191 & 20 & 34541.84 & & & & \\
\hline 2992.564 & 5 & 33406.42 & & & & & & 2891.518 & 2 & 34573.77 & & & & \\
\hline 2991.152 & 3 & 33422.19 & & & & & & 2890.576 & 5 & 34585.04 & & & & \\
\hline 2990.672 & 10 & 33427.55 & $8771_{s / 2}^{\circ}-42198_{5 / 2}$ & & & & & 2888.814 & $4 h$ & 34606.13 & & & & \\
\hline 2989.922 & 2 & 33435.94 & & & & & & 2885.047 & 5 & 34.651 .32 & & & & \\
\hline 2988.258 & 2 & 33454.55 & & & & & & 2884.289 & 2 & 34660.42 & & & & \\
\hline 2988.229 & 2 & 33454.88 & & & & & & 2882.917 & 10 & 34676.92 & & & & \\
\hline 2986.739 & 3 & 33471.57 & & & & & & 2879.152 & $70 h$ & 34722.26 & & & & \\
\hline 2985.519 & 10 & 33485.24 & & & & & & 2877.743 & 4 & 34.739 .26 & & & & \\
\hline 2984.834 & 40 & 33492.93 & & & & & & 2876.873 & 10 & 34749.77 & & & & \\
\hline 2982.889 & 10 & 33514.77 & & & & & & 2875.440 & 4 & 34767.08 & & & & \\
\hline 2979.637 & 30 & 33551.34 & & & & & & 2872.702 & $200 h$ & 34800.22 & & & & \\
\hline 2979.034 & 1 & 33558.13 & & & & & & 2871.319 & 2 & 34816.98 & & & & \\
\hline 2976.749 & 10 & 33583.89 & & & & & & 2869.725 & 4 & 34836.32 & & & & \\
\hline 2976.545 & 2 & 33586.20 & $8771_{5 / 2}^{\circ}-42357_{3 / 2}$ & & & & & 2868.461 & 1 & 34851.67 & & & & \\
\hline 2976.100 & 2 & 33591.22 & & & & & & 2866.840 & 20 & 34871.37 & & & & \\
\hline 2975.496 & 20 & 33598.04 & & & & & & 2865.069 & 1 & 34892.93 & & & & \\
\hline 2974.006 & 1 & 33614.87 & & & & & & 2864.757 & 300 & 34896.73 & & & & \\
\hline 2973.218 & $3000 a$ & 33623.78 & $0_{\tau^{\prime} / 2}^{\circ}-33623_{\tau_{7 / 2}}$ & $\Delta \mathrm{J}=0$ & 1.14 & 0.92 & * & 2863.012 & 10 & 34918.00 & & & & \\
\hline 2970.694 & 15 & 33652.34 & & & & & & 2854.166 & $1000 a$ & 35026.21 & $0_{7 / 2}^{\circ}-35026_{5 / 2}$ & SI & 1.45 & 0.30 \\
\hline 2968.688 & 2 & 33675.08 & & & & & & 2853.889 & 2 & 35029.61 & $8771_{5 / 2}^{\circ}-43800_{3 / 2}$ & & & \\
\hline 2965.059 & 10 & 33716.30 & & & & & & 2852.644 & 2 & 35044.90 & & & & \\
\hline 2964.592 & 2 & 33721.61 & & & & & & 2851.256 & 3 & 35061.96 & & & & \\
\hline 2964.494 & $20 w$ & 33722.72 & & & & & & 2848.169 & $4 h$ & 35099.96 & & & & \\
\hline 2962.314 & 10 & 33747.54 & & & & & & 2847.088 & 5 & 35113.29 & & & & \\
\hline 2961.749 & 40 & 33753.97 & & & & & & 2845.514 & 1 & 35132.71 & & & & \\
\hline 2957.287 & $20 h$ & 33804.90 & & & & & & 2842.812 & 1 & 35166.10 & & & & \\
\hline 2955.508 & 20 & 33825.25 & $8771_{5 / 2}^{\circ}-42596_{5 / 2}$ & & & & & 2842.225 & $2 h$ & 35173.36 & & & & \\
\hline 2953.910 & 10 & 33843.55 & $17454_{13 / 2}-51298^{\circ}{ }_{13 / 2}$ & & & & & 2841.283 & 15 & 35185.02 & & & & \\
\hline 2950.542 & $2 h$ & 33882.18 & & & & & & 2841.123 & $200 a$ & 35187.00 & $8771_{5 / 2}^{\circ}-43958_{5 / 2}$ & & & \\
\hline 2949.502 & 100 & 33894.12 & & & & & & 2840.657 & 2 & 35192.78 & & & & \\
\hline 2943.416 & 5 & 33964.20 & & & & & & 2839.279 & 3 & 35209.86 & $8771_{5 / 2}^{\circ}-4.3981_{7 / 2}$ & & & \\
\hline 2943.101 & 1 & 33967.84 & & & & & & 2837.841 & 10 & 35227.70 & & & & \\
\hline 2943.000 & 3 & 33969.00 & & & & & & 2837.294 & 10 & 35234.49 & & & & \\
\hline 2942.857 & 4 & 33970.65 & & & & & & 2835.098 & $80 a$ & 35261.78 & $0^{\circ}{ }_{7 / 2}-35261_{5 / 2}$ & & & \\
\hline 2942.528 & 100 & 33974.45 & & & & & & 2833.055 & 2 & 35287.21 & & & & \\
\hline 2940.755 & 3 & 33994.93 & & & & & & 2832.804 & 10 & 35290.33 & & & & \\
\hline 2940.087 & 4 & 34002.66 & & & & & & 2832.596 & 10 & 35292.92 & $15271_{15 / 2}-50563^{\circ}{ }_{15 / 2}$ & & & \\
\hline 2939.292 & 20 & 34011.85 & $17454_{13 / 2}-51466^{\circ}{ }_{13 / 2}$ & & & & & 2832.316 & 5 & 35296.41 & & & & \\
\hline 2935.699 & 50 & 34053.48 & & & & & & 2831.037 & 30 & 35312.36 & & & & \\
\hline 2934.222 & 9 & 34070.62 & & & & & & 2830.739 & 4 & 35316.07 & & & & \\
\hline 2932.966 & $1000 a$ & 34085.21 & $0_{7 / 2}^{\circ}-34085_{5 / 2}$ & SI & 1.14 & 0.95 & $*$ & 2827.187 & 1 & 35360.44 & & & & \\
\hline 2931.093 & 400 & 34106.99 & $16456_{17 / 2}-50563^{\circ}{ }_{15 / 2}$ & $\mathrm{~T}$ & 1.18 & & & 2826.690 & $50 a$ & 35366.66 & & & & \\
\hline
\end{tabular}


APPENDIX - Spectral Lines of Neutral Thulium-Continued

\begin{tabular}{|c|c|c|c|c|c|c|c|c|c|}
\hline \multirow{2}{*}{$\begin{array}{c}\text { Wave- } \\
\text { length } \\
\text { in air } \\
\AA\end{array}$} & \multirow[t]{2}{*}{ Intensity } & \multirow{2}{*}{$\begin{array}{l}\text { Wave- } \\
\text { number } \\
\mathrm{cm}^{-1}\end{array}$} & \multirow{2}{*}{ Classification } & \multirow{2}{*}{\begin{tabular}{ccc}
\multicolumn{3}{c}{ Zeeman effect } \\
type $\quad \mathrm{f}$ & Je
\end{tabular}} & \multirow{2}{*}{$\begin{array}{l}\text { Wave- } \\
\text { length } \\
\text { in air } \\
\AA\end{array}$} & \multirow[t]{2}{*}{ Intensity } & \multirow{2}{*}{$\begin{array}{c}\text { Wave- } \\
\text { number } \\
\mathrm{cm}^{-1}\end{array}$} & \multirow[t]{2}{*}{ Classification } & \multirow{2}{*}{\begin{tabular}{lll}
\multicolumn{3}{c}{ Zeeman effect } \\
type $\mathrm{f}$
\end{tabular}} \\
\hline & & & & & & & & & \\
\hline 2821.626 & 15 & 35430.13 & \multirow{10}{*}{$15587_{11 / 2}-51298^{\circ}{ }_{13 / 2}$} & & 2651.570 & 7 & 37702.28 & \multirow{8}{*}{$\begin{array}{l}0_{7^{\prime} / 2}^{\circ}-37711_{9 / 2} \\
0_{7_{72}}^{\circ}-37724_{5 / 2}\end{array}$} & \\
\hline 2821.558 & 1 & 35430.98 & & & 2651.214 & 3 & 37707.34 & & \\
\hline 2819.552 & 2 & 35456.19 & & & 2651.173 & 20 & 37707.93 & & \\
\hline 2818.285 & 2 & 35472.13 & & & 2650.950 & 10 & 37711.10 & & \\
\hline 2811.972 & 1 & 35551.76 & & & 2650.628 & 3 & 37715.68 & & \\
\hline 2805.574 & 10 & 35632.83 & & & 2649.986 & 4 & 37724.82 & & \\
\hline 2803.727 & 1 & 35656.30 & & & 2633.727 & 2 & 37957.69 & & \\
\hline 2799.468 & 5 & 35710.55 & & & 2626.054 & 3 & 38068.59 & & \\
\hline 2798.865 & 4 & 35718.24 & & & 2622.467 & 3 & 38120.66 & $0_{7 / 2}^{\circ}-38120_{9 / 2}$ & \\
\hline 2795.122 & 1 & 35766.07 & & & 2621.936 & 10 & 38128.38 & $0_{7 / 2}^{\circ / 2}-38128_{5 / 2}^{\circ / 2}$ & \\
\hline 2790.721 & 10 & 35822.47 & \multirow{4}{*}{$15587_{11 / 2}-51466^{\circ}{ }_{13 / 2}$} & & 2601.092 & 8 & 38433.91 & \multirow{3}{*}{$\begin{array}{l}0_{7 / 2}^{\circ}-38433_{5 / 2} \\
0_{7 / 2}^{\circ}-38502_{9 / 2}\end{array}$} & \\
\hline 2789.810 & 2 & 35834.17 & & & 2596.489 & 10 & 38502.04 & & \\
\hline 2786.329 & 2 & 35878.93 & & & 2579.205 & 1 & 38760.03 & & \\
\hline 2783.461 & 5 & 35915.90 & & & 2564.058 & 5 & 38988.99 & \multirow{4}{*}{$0_{7 / 2}^{\circ}-3916 \mathrm{l}_{5 / 2}$} & \\
\hline 2775.875 & 3 & 36014.05 & \multirow{6}{*}{$8771_{s / 2}^{\circ}-44891_{7 / 2}$} & & 2553.514 & 10 & 39149.97 & & \\
\hline 2773.898 & 15 & 36039.71 & & & 2552.764 & 50 & 39161.48 & & \\
\hline 2768.840 & 1 & 36105.55 & & & 2552.263 & 2 & 39169.16 & & \\
\hline 2767.731 & 4 & 36120.01 & & & 2551.115 & 5 & 39186.79 & \multirow{3}{*}{$0_{7 / 2}^{\circ}-39206_{9 / 2}$} & \\
\hline 2764.848 & 8 & 36157.67 & & & 2549.810 & 5 & 39206.84 & & \\
\hline 2763.023 & 1 & 36181.55 & & & 2541.776 & 5 & 39330.76 & & \\
\hline 2761.938 & 1 & 36195.77 & \multirow{10}{*}{$15271_{15 / 2}-51466^{\circ}{ }_{13 / 2}$} & & 2539.469 & 2 & 39366.49 & \multirow{10}{*}{$0_{7 / 2}^{\circ}-39560_{\tau / 2}$} & \\
\hline 2759.450 & 8 & 36228.40 & & & 2535.432 & 5 & 39429.16 & & \\
\hline 2753.176 & 50 & 36310.95 & & & 2534.719 & 50 & 39440.25 & & \\
\hline 2752.182 & 50 & 36324.07 & & & 2533.181 & 1 & 39464.20 & & \\
\hline 2751.646 & $2 h$ & 36331.14 & & & 2533.144 & 5 & 39464.77 & & \\
\hline 2748.870 & 2 & 36367.83 & & & 2527.542 & 5 & 39552.24 & & \\
\hline 2747.158 & 4 & 36390.49 & & & 2527.275 & 2 & 39556.42 & & \\
\hline 2739.775 & $2 h$ & 36488.55 & & & 2527.022 & $10 r$ & 39560.37 & & \\
\hline 2709.625 & 15 & 36894.54 & & & 2525.723 & 2 & 39580.72 & & \\
\hline 2708.020 & 3 & 36916.40 & & & 2520.768 & $5 h$ & 39658.52 & & \\
\hline 2692.207 & 10 & 37133.22 & \multirow{10}{*}{$0_{{ }^{\prime} / 2}^{\circ}-37576_{9 / 2}$} & & 2520.655 & 2 & 39660.30 & \multirow{10}{*}{$0_{7 / 2}^{\circ}-39768_{9 / 2}$} & \\
\hline 2691.342 & 7 & 37145.16 & & & 2520.398 & 1 & 39664.34 & & \\
\hline 2681.860 & $3 h$ & 37276.48 & & & 2517.694 & 1 & 39706.94 & & \\
\hline 2679.918 & 3 & 37303.49 & & & 2513.778 & $5 r$ & 39768.79 & & \\
\hline 2673.342 & 1 & 37395.25 & & & & & & & \\
\hline 2669.591 & 1 & 37447.79 & & & & & & & \\
\hline 2664.616 & 10 & 37517.70 & & & & & & & \\
\hline 2661.810 & 1 & 37557.25 & & & & & & & \\
\hline 2660.417 & 20 & 37576.91 & & & & & & & \\
\hline 2657.203 & 1 & 37622.36 & & & & & & & \\
\hline
\end{tabular}

